\title{
Translational Studies on Grafting Materials in Alveolar cleft repair
}

Citation for published version (APA):

Kamal, M. (2018). Translational Studies on Grafting Materials in Alveolar cleft repair: Experimental Applications in Preclinical Sciences. [Doctoral Thesis, Maastricht University]. Maastricht University. https://doi.org/10.26481/dis.20181024mk

Document status and date:

Published: 01/01/2018

DOI:

10.26481/dis.20181024mk

Document Version:

Publisher's PDF, also known as Version of record

\section{Please check the document version of this publication:}

- A submitted manuscript is the version of the article upon submission and before peer-review. There can be important differences between the submitted version and the official published version of record.

People interested in the research are advised to contact the author for the final version of the publication, or visit the DOI to the publisher's website.

- The final author version and the galley proof are versions of the publication after peer review.

- The final published version features the final layout of the paper including the volume, issue and page numbers.

Link to publication

\footnotetext{
General rights rights.

- You may freely distribute the URL identifying the publication in the public portal. please follow below link for the End User Agreement:

www.umlib.nl/taverne-license

Take down policy

If you believe that this document breaches copyright please contact us at:

repository@maastrichtuniversity.nl

providing details and we will investigate your claim.
}

Copyright and moral rights for the publications made accessible in the public portal are retained by the authors and/or other copyright owners and it is a condition of accessing publications that users recognise and abide by the legal requirements associated with these

- Users may download and print one copy of any publication from the public portal for the purpose of private study or research.

- You may not further distribute the material or use it for any profit-making activity or commercial gain

If the publication is distributed under the terms of Article $25 \mathrm{fa}$ of the Dutch Copyright Act, indicated by the "Taverne" license above, 

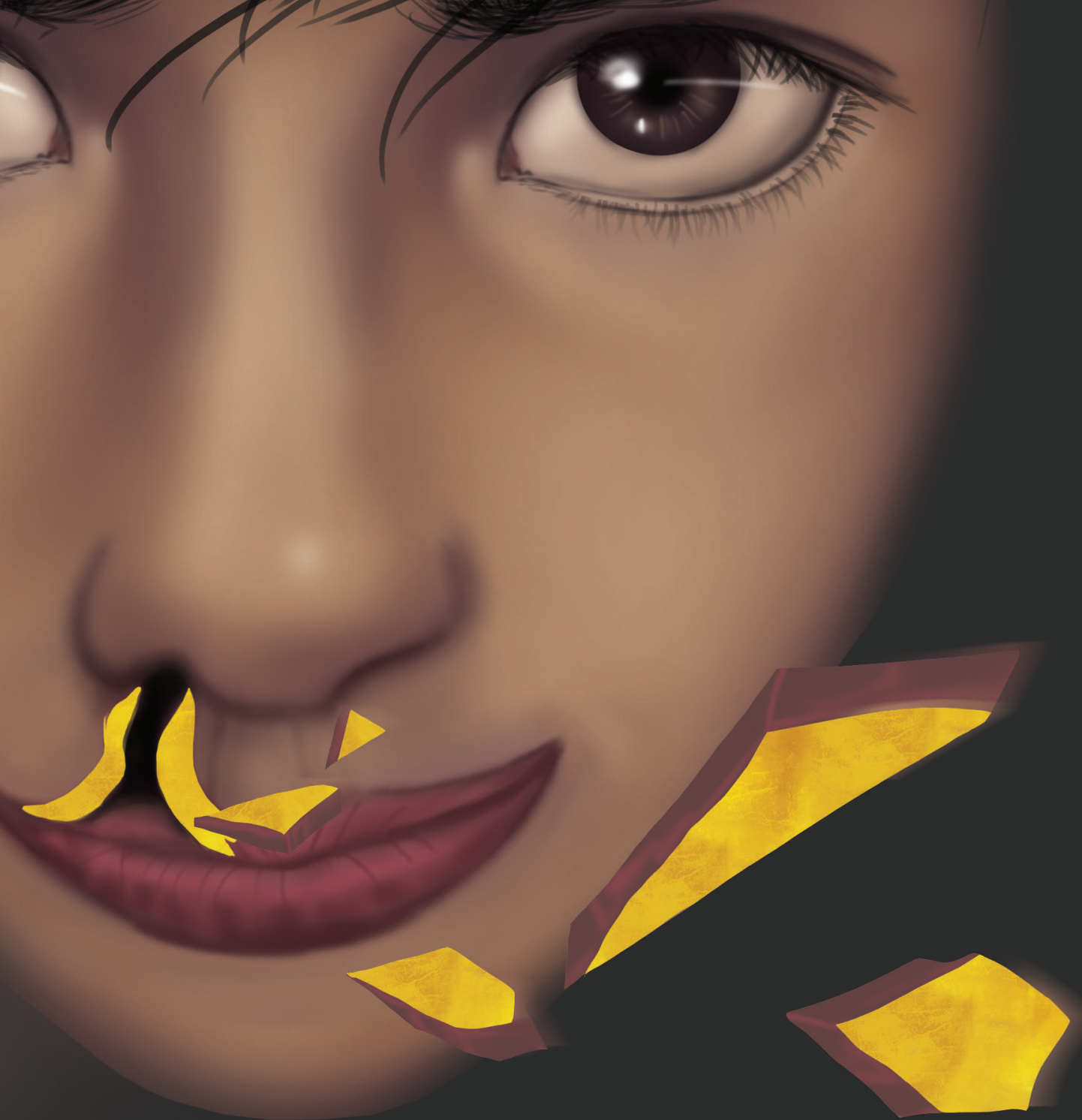

Translational Studies on Grafting Materials in Alveolar Cleft Repair

Experimental Applications in Preclinical Sciences 


\section{TRANSLATIONAL STUDIES ON GRAFTING MATERIALS IN ALVEOLAR CLEFT REPAIR}

Experimental Applications in Preclinical Sciences

Mohammad Kamal 
The research presented in this thesis was performed at:

Department of Cranio-Maxillofacial Surgery and GROW School for Oncology and Developmental Biology, Maastricht University Medical Center, Maastricht, The Netherlands. Department of Oral and Maxillofacial Surgery, RWTH Aachen University Hospital, Aachen, Germany. Department of Surgical Sciences, Kuwait University Health Sciences Center, Kuwait.

\section{In collaboration with:}

Department of Experimental Molecular Imaging, RWTH Aachen University, Aachen, Germany. Animal Resources Centre, Kuwait University Health Sciences Center, Kuwait. Department of Community Medicine and Behavioral Sciences, Kuwait University Health Sciences Center, Kuwait.Department of Surgical Sciences, Kuwait University Health Sciences Center, Kuwait. Maxillofacial Unit, Huddersfield Royal Infirmary, Huddersfield, United Kingdom. Institute for Laboratory Animal Science and Experimental Surgery, RWTH Aachen University, Aachen, Germany.

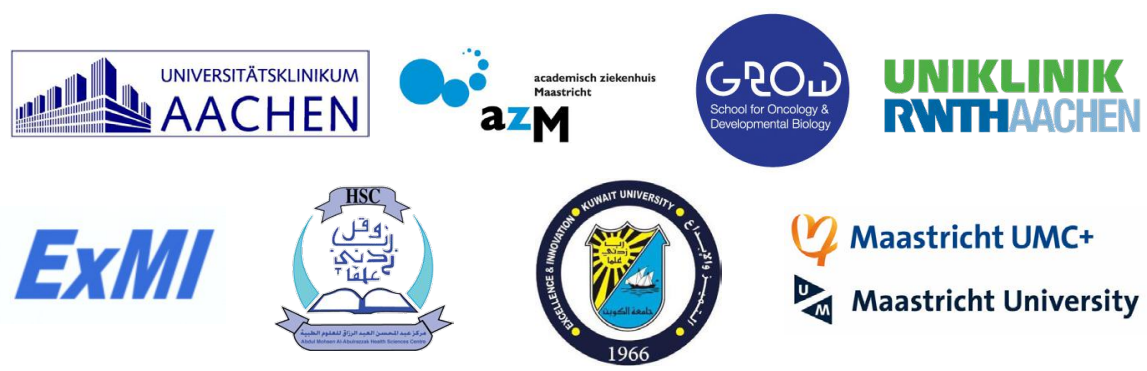

Mohammad Kamal

Translational Studies on Grafting Materials in Alveolar Cleft Repair.

Experimental Applications in Preclinical Sciences.

Thesis Maastricht University Medical Center, Maastricht, the Netherlands

ISBN: 9789463233163

(c) Mohammad Kamal, Aachen, Germany, 2018

Cover design and layout: @ evelienjagtman.com

Production: Gildeprint Drukkerijen

This research project was supported by the services provided by facilities at RWTH Aachen University Hospital and Kuwait University Health Sciences Center. 


\title{
TRANSLATIONAL STUDIES ON GRAFTING MATERIALS IN ALVEOLAR CLEFT REPAIR
}

\author{
Experimental Applications in Preclinical Sciences
}

\section{DISSERTATION}

to obtain the degree of Doctor at the Maastricht University, on the authority of the Rector Magnificus, Prof. dr. Rianne M. Letschert

in accordance with the decision of the Board of Deans,

to be defended in public on

Wednesday October 24, 2018 at 16:00 hours

by

Mohammad Kamal

born in Kuwait on 18 December 1978 


\section{Supervisors:}

Prof. dr. dr. P.A.W.H. Kessler

\section{Co-supervisors:}

dr. dr. B. Lethaus, RWTH Aachen University, Germany

Prof. dr. L. Andersson, Kuwait University, Kuwait and Mälmo University, Sweden

\section{Assessment Committee:}

Prof. dr. L.W. van Rhijn (Chairman)

Prof. dr. A.G. Becking, University of Amsterdam

Prof. dr. A. Herrler

Prof. dr. dr. F. Hölzle, RWTH Aachen University, Germany

Prof. dr. H.G. Brunner

* The doctoral research (Ph.D.) has been carried out in the context of an agreement on joint doctoral supervision between RWTH Aachen University Hospital, Department of Oral and Maxillofacial Surgery, Germany, and Maastricht University Medical Center, Department of Cranio-Maxillofacial Surgery and GROW School for Oncology and Developmental Biology, the Netherlands. 
To those who provided me with utmost support during my academic and clinical endeavour ...

To those who never gave up believing in the power of persistence...

To those who nourish the human mind and prosper it ...

To my beloved wife Fatmah, children, and family ...

I affectionately dedicate this thesis ... 
To my father; you would have been proud. 


\section{CONTENTS}

CHAPTER 1 Prologue-General Introduction 9

CHAPTER 2 Volumetric Comparison of Autogenous Bone and Tissue-

Engineered Bone Replacement Materials in Alveolar Cleft Repair: A Systematic Review and Meta-Analysis

CHAPTER 3 Comparison of Bone Grafts from Various Donor Sites in Human Bone Specimens

CHAPTER 4 New Bone Formation Around Xenogenic Dentin Grafts to Rabbit Tibia Marrow

CHAPTER 5 Bone Regeneration with Composite In-Situ Formed NonDemineralized Xenogenic Dentin and Biphasic Tricalcium Phosphate/Hyroxyapetite Mixture in Rabbit Calvarial Defects

CHAPTER 6 A Rabbit Model for Experimental Alveolar Cleft Grafting

CHAPTER 7 Bone Regeneration Using Composite Non-Demineralized Xenogenic Dentin with Beta-Tricalcium Phosphate in Experimental Alveolar Cleft Repair in a Rabbit Model

CHAPTER 8 Epilogue-General Discussion and Future Perspectives

Summary

Publications

Curriculum Vitae

Gratitude 


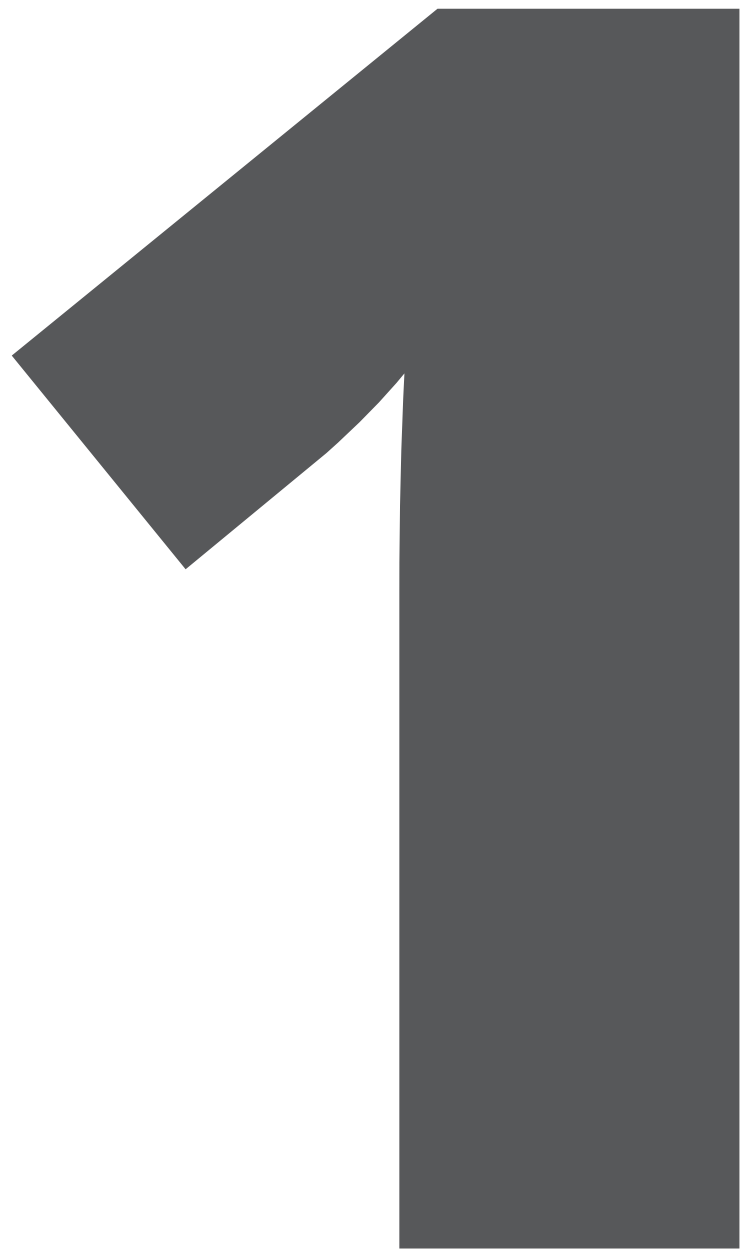




\section{Prologue}

General Introduction 



\section{PROLOGUE}

\section{Alveolar Cleft Repair - \\ Clinical Perspective Within a Comprehensive Approach}

Cleft lip and palate is the most common congenital facial malformation occurring as a result of non-fusion of the primary palate during the $4^{\text {th }}$ to $12^{\text {th }}$ weeks of gestation with an incidence as high as 1 per 500 births depending on the geographic location and the demography of the population (Figure 1). 1,2 Epidemiological studies on the incidence of cleft lip and palate by its Global Burden of Disease report that the highest incidence of this congenital malformation appears to occur among Asians (0.82-4.04 per 1000 live births), with intermediate incidence in Caucasians (0.9-2.69 per 1000 live births) and lowest among Africans (0.18-1.67 per 1000 live births). ${ }^{3}$ In 75\% of patients with cleft lip and palate, this orofacial malformation leads to a skeletal cleft in the alveolus of the maxillary arch in the region of the lateral incisor and canine tooth extending to the nasal mucosa. 4,5 The alveolar cleft defect results in a loss of the continuity of the dental arch, missing teeth, communication between the oral and nasal cavity, resulting in hyper-nasal speech, nasal deformity, and collapse of the alveolar arch and the lip support. 4,6 This results in a significant physical disfigurement of the face, and further entails significant psychological distress to both the growing child and the family, making these patients more prone to depression, anxiety, dissatisfaction with their facial appearance, social neglect, and increased physical and financial burden for the family overall (Figure 2). 1,7-9

Alveolar cleft repair is performed by a skeletal augmentation procedure, by which the congenital alveolar cleft defect is filled with a biologic material that physiologically interacts with native bone in the recipient site via complex molecular signaling and interactions leading to the promotion of new bone formation, osteogenic remodeling, and eventually replacement by functionally viable bone. ${ }^{5}$ The goal of alveolar cleft repair is to establish bony continuity of the alveolar ridge in the maxilla, stabilize the maxillary segments during orthodontic treatment, seal the oro-nasal communication, support the nasal alar cartilage, form an ideal alveolar morphology, and establish a favorable anatomy for the eruption of teeth through the reconstructed area, and the ability to perform functional orthodontic movement, including sufficient quality and quantity of alveolar bone with adequate attached gingiva for future dental implant placement (Figure 3). 2, 4, 6, 10,11 The repair of alveolar cleft defect sites should allow teeth movement into correct anatomical position by means of natural eruption, orthodontic therapy, or, after growth has been completed, replacement of missing teeth by endosseal dental implants. Alveolar cleft repair and restoration of the dental arch also lead to improvement of the facial aesthetic appearance, increasing lip support, mimic of the facial expression muscles, improvement in phonation and deglutition, and overall improvement in the child's positive self-attributes and psychological well-being. 1,7-9 
Figure 1.
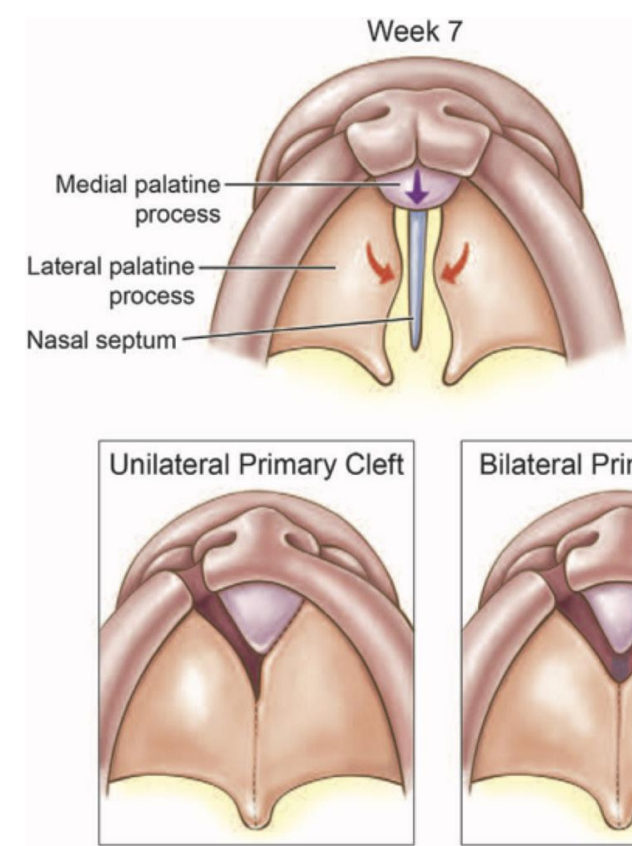

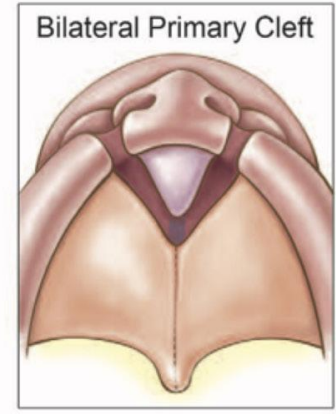

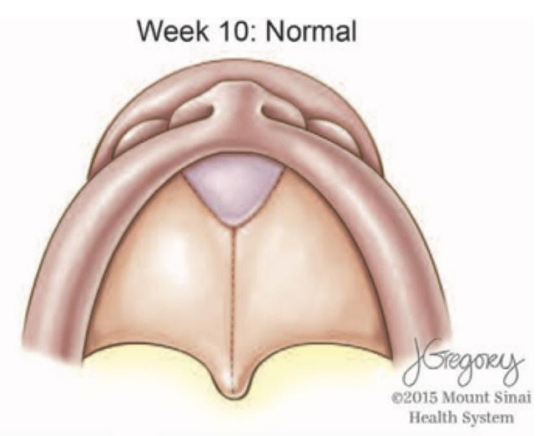

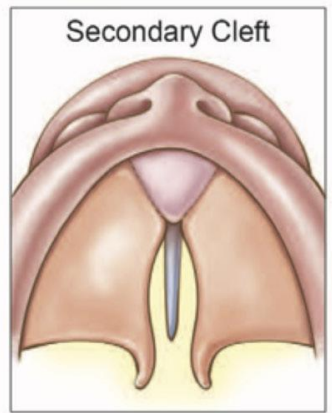

(Above, left) Week 7: the medial palatine process is derived from the frontonasal eminence and lateral palatine process from the first branchial arch maxillary prominences. (Above, right) Week 10: fusion of the medial palatine process, lateral palatine processes, and nasal septum gives oronasal separation, with the medial palatine process becoming the primary palate and lateral palatine processes the secondary palate. (Below, left) Unilateral primary cleft: caused by failure of fusion of the medial and lateral palatine processes on one side. (Below, center) Bilateral primary cleft: caused by failure of fusion of the median palatine process to the fused lateral palatine processes. (Below, right) Secondary cleft: caused by failure of the lateral palatine processes to fuse to each other. (Reprinted with permission from WOLTERS KLUWER HEALTH, INC. Weissler EH, Paine KM, Ahmed MK, Taub PJ. Alveolar Bone Grafting and Cleft Lip and Palate: A Review. Plastic and reconstructive surgery. 2016 Dec 1;138(6):1287-95.)

Timing of the alveolar cleft repair is subject to scientific debate, but the majority of the centers worldwide perform the alveolar bone repair as a secondary bone grafting procedure in the mixed dentition stage, between the age of 8 and 11. Grafting at this age has shown to have minimal restrictive effect on the growth of the maxilla, which is usually completed by the age of 8 , and prior to eruption of the canine tooth, which erupts at around 10 years of age (Figure 4). ${ }^{5}$ Several studies have compared the efficacy on performing the repair, either at an earlier stage "early secondary alveolar bone grafting", or at later age "late secondary alveolar bone grafting". 

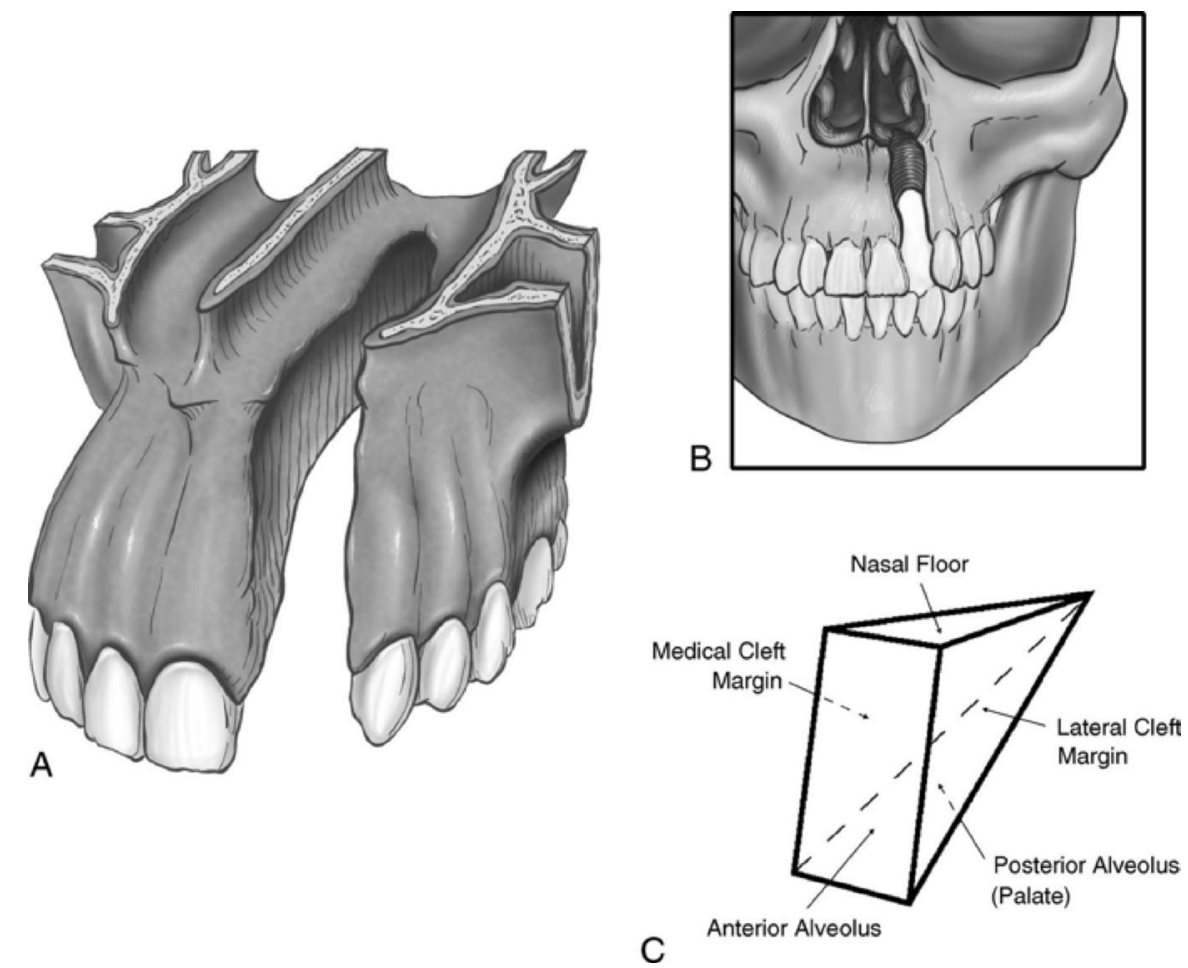

(A-C) The alveolar and hard palate cleft should be viewed as a three-dimensional defect resembling a triangle or pyramid. (Reprinted with permission from WOLTERS KLUWER HEALTH, INC. Craven C, Cole P, Hollier L Jr, Stal S. Ensuring success in alveolar bone grafting: a three-dimensional approach. J Craniofacial Surgery 2007;18(4):855-859)

\section{Bone Grafting -}

\section{A Synchronous Reciprocation Between Bone Formation and Remodelling}

Bone is a dynamic tissue made of organic and inorganic components with a unique healing capacity to regenerate new bone without scarring. Bone formation occurs through endochondoral or intramembranous ossification. Endochondral bone formation begins with the formation of a cartilage template followed by graduate ossification, and it occurs mostly in long bones such as the tibia, femur, radius, and in growth plates. Intramembranous bone formation starts by formation of clusters of dense mesenchymal stem cells, which then differentiate into osteobalsts and directly produce osteoid, for example in the skull and ribs. Smaller bone defects can heal spontaneously without intervention, but larger bone defects do not spontaneously heal, and require an interventional procedure to enhance bone regeneration in a process called bone grafting or augmentation procedure. 


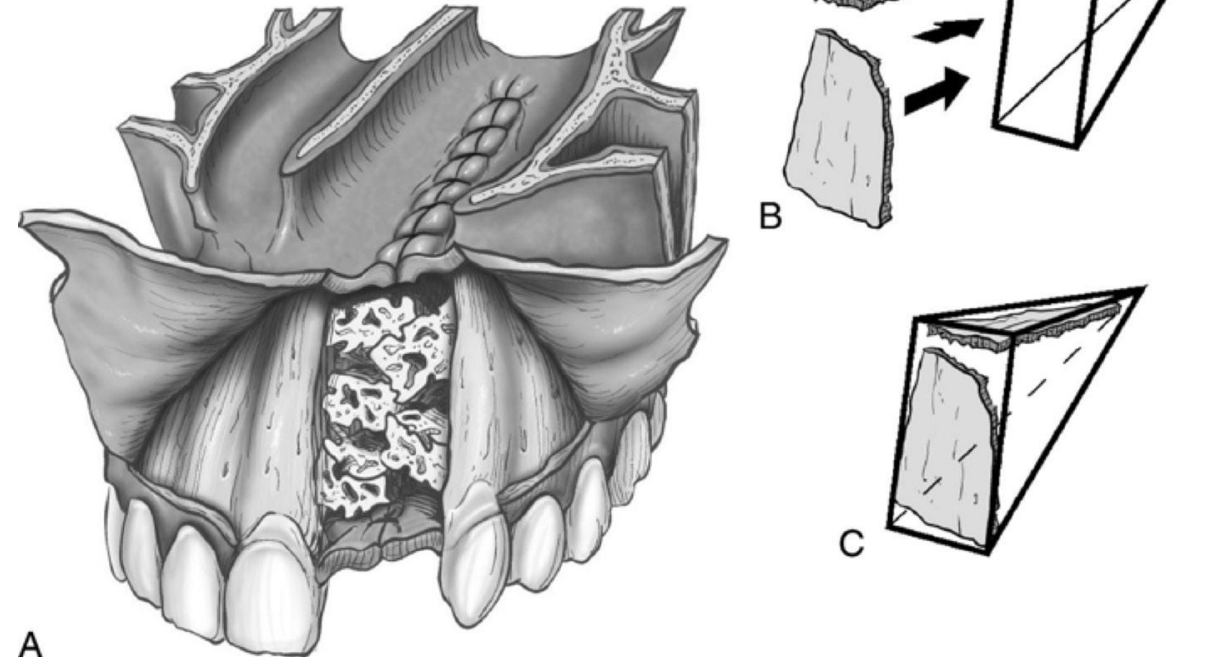

(A) The alveolar cleft after packing with cortical and cancellous bone. (B,C) Cortical bone reinforcing the roof of the cleft (nasal floor) and the anterior wall of the alveolus. (Reprinted with permission from WOLTERS KLUWER HEALTH, INC. Craven C, Cole P, Hollier LJr, Stal S. Ensuring success in alveolar bone grafting: a three-dimensional approach. J Craniofacial Surgery 2007;18(4):855-859)

Proper grafting of alveolar cleft defects is an essential step to re-establish the dental arch in patients with a cleft palate. In order to achieve the goals of alveolar bone grafting, a sufficient amount of viable bone must be established. ${ }^{2}$ This depends on the type of the grafting material that aims to restore form, volume and the functional environment of a skeletal biological medium that would allow for the eruption of the permanent teeth. In order to meet these biological functions, an optimal bone grafting material should promote bone healing and osseous regeneration by osteoinduction, osteoconduction, a controlled resorption rate, and enhancement of vascularisation in the grafted region. In addition, operation related technical features like easiness of handling and application, and cost-effectiveness are important. ${ }^{12,13}$ Autologous bone is the gold standard for grafting in many types of bone defects and deficiencies in the craniofacial region, but an increasing number of various bone replacement materials are being used in clinical practice. ${ }^{12-15}$

Previous studies have evaluated several structural bone qualities (macro- and micro-architecture) along with biomaterial characteristics such as bone mineral density to represent the mechanical strength of bone. These factors were proposed as the main predictors of bone quality in bone grafting. ${ }^{16-20}$ Additional studies have investigated the qualitative and quantitative structural morphology for evaluating bone grafts and regenerative bone 
substitute materials during healing bone healing and integration of the grafts to answer questions related to the heterogeneity of bone graft resorption and the maintenance of bone volume between intramembranous and endochondral bone. ${ }^{21,22}$ Some researchers have found that volume maintenance of various bone grafts was affected by dynamic microstructural interactions, along with local grafting site factors like intact periosteum, mechanical fixation of the graft, and the similarity between grafted and recipient bone to allow graft incorporation and a controlled rate of resorption. ${ }^{21-23}$

Figure. 4.
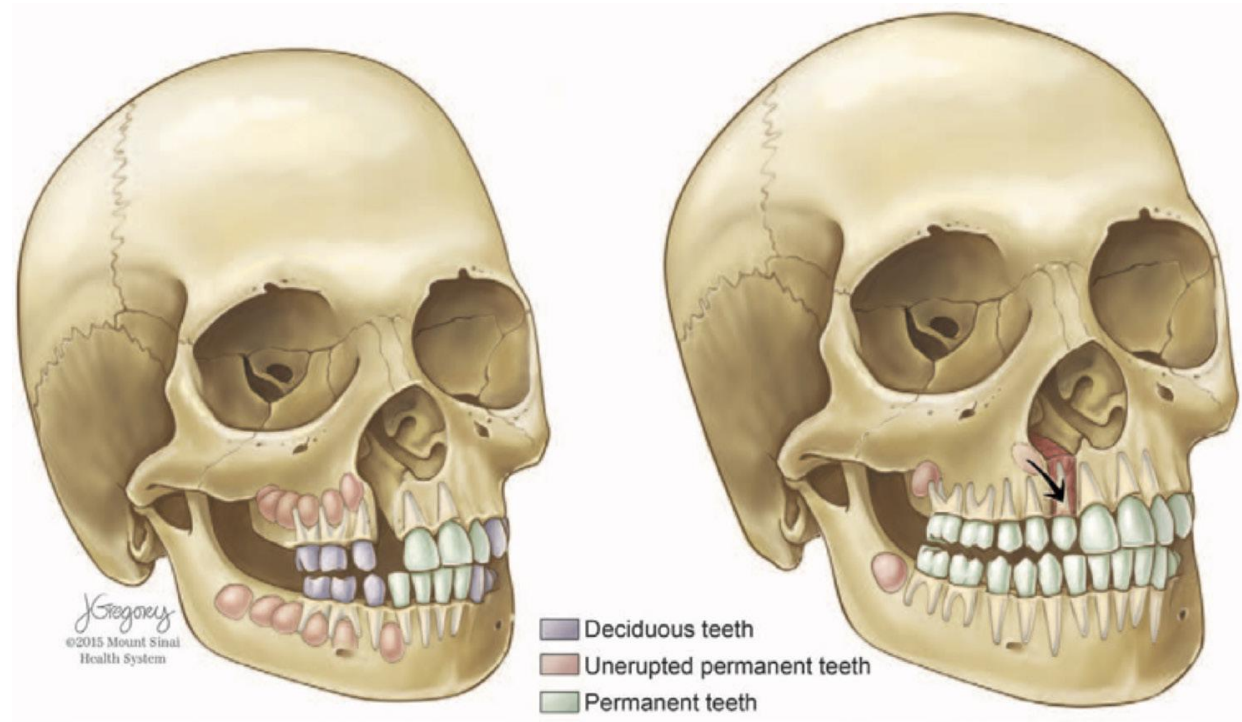

(Left) Alveolar bone grafting is optimally performed in the stage of mixed dentition. (Right) Descent of the cleft-adjacent canine into the alveolar graft puts functional stress on the graft to increase graft take and reestablishes normal dentition. (Reprinted with permission from WOLTERS KLUWER HEALTH, INC. Weissler EH, Paine KM, Ahmed MK, Taub PJ. Alveolar Bone Grafting and Cleft Lip and Palate: A Review. Plastic and reconstructive surgery. 2016 Dec 1;138(6):1287-95.)

\section{Autogenous Bone Grafts}

Autogenous bone grafts are the current standard of care to graft alveolar cleft defects. It can be harvested from various anatomical regions including the iliac crest, calvarium, mandibular symphysis, mandibular ramus, and the tibia. Each donor site carries its unique risks and morbidities (Figure 5). ${ }^{24,25}$ Autogenous bone of various intramembranous or endochondral embryonic origins offer heterogeneous bone qualities and distinctive biomechanical and structural features, making them selectively prone to resorption and loss of graft volume over time due to inherent morphologic properties. 12, 19, 20, 26, 27 
However, many clinical drawbacks are associated with autogenous bone grafts. These include a limited available bone supply at distinct anatomical regions, the need for second surgery with subsequent donor site morbidity and its effect on the patient's quality of life, the reliability of the anatomical landmarks, the increased operative time and costs, patient's acceptance, along with several clinical reports on an increased resorption rate with unpredictable graft stability. ${ }^{13-15,20,24-31}$ Even worse, an extensive resorption of the autogenous bone graft can sometimes be seen. ${ }^{22,26,32,33}$ Given the reported disadvantages and morbidities associated with autogenous bone, along with technological advances in the field of regenerative bone substitute materials, many researchers have questioned its current role as a gold standard. ${ }^{24,26,27}$

Figure 5. Donor sites commonly utilized for harvesting non-vascularised bone grafts for augmentation procedures in craniofacial surgery.

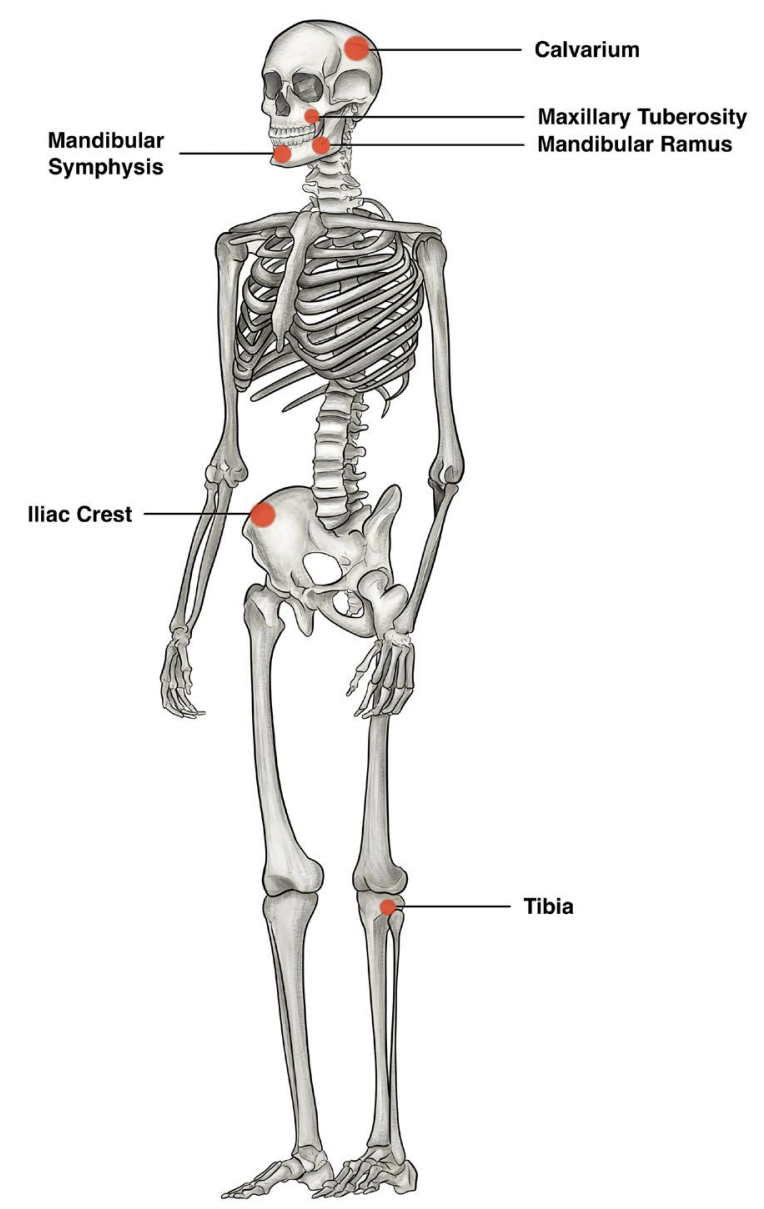




\section{Regenerative Bone Substitute Materials}

To overcome clinical shortcomings associated with autogenous bone, ongoing attempts in the field of biomedical sciences have been made in order to develop a suitable bone substitute material that would effectively enhance bone regeneration, be biocompatible with absence of toxicity, and maintain the grafting volume with controlled rate of resorbability. Furthermore, this material may not harm the immunogenicity, should be dimensionally stable under stress, and eventually be replacable by viable bone. ${ }^{12,19}$ Several regenerative bone substitute materials have been reported in the literature as suitable bone replacement materials for craniofacial reconstruction including processed allografts 34, 35, xenografts ${ }^{36}$, and alloplasts such as bioactive ceramics like hydroxyapatite and tricalcium phosphate ${ }^{34,37-39}$, and polymer based materials like polyether ether ketone and fiber-reinforced bioglass. ${ }^{38,40}$

A current review of the literature revealed the utilization of multiple bone substitute materials for bone regeneration. ${ }^{13,14,30,31,41,42}$ These materials can be categorized into different groups (Table 1) based on their composition including:

1. Growth Factors:

Biological factors based on molecular cellular interactions leading to enhanced osteogensis. They include Bone Morphogenic Proteins (BMP), Platelet Rich Plasma (PRP), and Platelet Derived Growth Factors (PDGF).

2. Enhanced Scaffolds and Cell Therapy (MSC, OB)/Growth Factors in combination:

Scaffolds which are utilized to promote osteoconduction. Scaffolds are usually impregnated with cellular components either with Mesenchymal Stem Cells (MSC) or Osteoblasts $(\mathrm{OB})$, which can also be treated with growth factors to further enhance bone formation.

3. Biocomposites and Hemostatic Agents:

Biocompatible synthetic materials or biological components that promote osteoconduction and enhance the formation of blood clots, leading to enhanced delivery of growth factors and promotion of osteogenesis. Biocompatible alloplastic materials include calcium phosphate and hydroxyapatite, whereas biologic hemostatic agents include fibrin glue and similar hemostatic agents.

However, many of the bone substitute materials are the result of very recent advances in biomedical sciences with various levels of efficacy and with limited documentation. Thus, improving surgical techniques, optimizing the quality of the existing materials and looking for novel and better bone-substitute materials is crucial in improving the clinical outcome. Further scientific work comparing grafting materials is needed to be able to recommend suitable alternative materials. 


\section{Calcium Phosphate Ceramics}

Calcium phosphate ceramics are well known biocompatible ceramics commonly used as bone substitute material given their biocompatibility and osteoconductive properties promoting new bone formation in various interventional studies in human and translational animal models. 34,35, 37-39, 43,44 Various forms of calcium phosphate bone substitute materials exist, but most clinical formulations are made of beta tri-calcium phosphate ( $\beta$-TCP) and calcium hydroxyapatite $(H A)$, the principle mineral component of bone. Both materials are proportionally combined to obtain a suitable balance between the predictable biodegradability offered by tri-calcium phosphate ( $\beta$-TCP), and the resiliency with improved mechanical properties offered by hydroxyapatite (HA). ${ }^{39,44}$ The grafting material has a crystalline structure and geometric porosity which allows it to have effective osteoconductive and osteointegrative properties, with excellent biocompatibility and biomechanical strength, which is obtained shortly after placement in a wet-body environment. This may make it suitable as a sole bone grafting substitute material, or in combination with additional grafting to promote graft stability and enhance its osteogenic potential.

\section{Dentin}

Dentin, a natural hard tissue organic component of teeth and similar in its chemical composition to bone, has also been investigated as an effective bone grafting substitute material. Mature dentin is composed of $70 \%$ mineralized inorganic material, $20 \%$ organic material, and $10 \%$ water in comparison to the composition of alveolar bone which is $60 \%$, $25 \%$, and $15 \%$ respectively. The physiochemical and biomechanical strength of skeletal hard tissue depends mostly on its inorganic composition, and the major inorganic content in dentin is composed of several types of calcium phosphates including beta-tricalcium (B-TCP) phosphate, amorphous calcium phosphate (ACP), octacalium phosphate (OCP), and hydroxyapatite $(\mathrm{HA})$.

Several previous experimental and interventional studies have demonstrated that delayed replantation of teeth with non-viable periodontal ligaments will fuse with bone in a process called ankylosis. ${ }^{45-50}$ The ankylosed teeth, which are mostly made of dentin, will gradually resorb and subsequently undergo osseous replacement in a process called replacement resorption, and it is mostly attributed to a bone remodelling process. $45,46,48,49,51-53$ Several studies have focused on this biological phenomenon to preserve alveolar ridge height and width by delayed replantation of teeth or decoronation of the tooth for retention in the alveolar process to be slowly replaced by bone, thus maintaining the volume of alveolar process and preventing bone resorption. 47,49,54,55 
Additional studies on human and in-vivo models have demonstrated that dentin promotes new bone regeneration with inherent osteoinductive and osteoconductive properties. ${ }^{36,46 \text {, }}$ 51-53,56-59 Several forms of allogenic and xenogenic dentin forms have been investigated as a potential bone replacement material including processed bovine dentin, allogenic freezedried dentin, demineralised dentin, and dried particulate dentine (chips and blocks). ${ }^{59}$ In our previous work conducted at Kuwait University, our results showed that dentin possesses mainly osteoconductive properties and promotes new bone formation when placed close to the native cortical bone in rabbit tibia, thus acting as an effective material for bone augmentation. 46, 51-53, 60 Dried dentin block xenografts have shown promising results with incorporation into native bone with minimal inflammation and slow pattern of graft resorption and replacement with bone over time. ${ }^{46,51-53,60}$ The pattern of dentin graft incorporation with a slow resorption rate may help to overcome a major issue associated with autogenous bone grafts, mainly the rapid resorption rate of the grafted bone and the possible need for reoperation and revision of the bone grafting procedure. Dentin is readily available from extracted human teeth and can be processed as particles or as blocks for grafting.

\section{Experimental Animal Models}

Animal models with alveolar cleft defects are considered appropriate as an experimental model for testing of clinical interventions, as they simulate the clinical scenario presented in human patients with alveolar cleft deformity. Development of alveolar clefts in experimental animals can be achieved, either surgically created or congenitally induced in utero during embryonic development. ${ }^{61-64}$ Several models have been proposed as alveolar cleft models to test bone replacement materials. These range from mice, rats, rabbits, cats, dogs, pigs, goats, sheep to monkeys. ${ }^{28,33,63,65-78}$

In recent years, attempts have been made to create more effective, economical and smaller animal models to create acceptable alveolar cleft defects. Despite the increasing number of descriptions of animal models for alveolar bone grafting material testing, most of the in vivo animal models using small animals are not useful to function as reliable alveolar cleft models simulating that in humans. This is mostly because of the limited anatomical size in these models which leads to difficulties in handling intraoral surgical procedures, and hence, a compromise in designing and establishing a sizable defect mimicking three-dimensional defects as seen in human patients. ${ }^{68,74,79}$ For this reason, rodent models have inherent limitations when compared to larger animal models. They have smaller longbones, a more fragile cortex, and do not show Haversian-type remodeling in the cortex. ${ }^{80}$ Rabbits are considered to be the largest animals in the small animals category, and hence, less susceptible to elaborate and exhaustive additional clearance requirements usually implemented by the central ethical committees. They are non-aggressive, easy to observe, 
have a quicker vital capacity in terms of gestation and maturity, and can be locally bred. 80-82 The histology of bone in rabbits is not similar to that of humans, and is a composite of dense Haversian bone and layers of primarily vascular longitudinal canals. ${ }^{80-82}$ However, similarities in bone mineral density and fracture toughness between rabbits and humans have been reported in the literature. ${ }^{81-83}$ An essential issue with rabbits is that they express rapid skeletal metabolism and an increased bone turnover rate, mostly cortical remodeling when compared to primates and some rodents. ${ }^{80-82}$ Thus, the rabbit model is an appropriate animal model for alveolar cleft experimental studies and to properly simulate the human in-vivo environment because the rabbit is reproducible, accurate, easy to house and handle, relatively easily anaesthetised, provides a large enough area for testing and is a properly sized mammal that can bear the trauma of surgery. ${ }^{81,82}$

\section{Methods of Quantitative Analysis of Bone Regeneration}

The molecular interaction between native bone and the grafting materials is considered of paramount importance in graft healing and promotion of bone formation. To quantify the bone regeneration potential of bone substitute materials, many non-invasive methods, such as micro-computed tomography, have been developed to observe the three-dimensional structures of these tissues. In addition to the histological observation of the bone-graft interface and healing pattern, these methods provide unique information on cellularity and dynamic indices of bone remodelling.

Preclinical micro-computed tomography $(\mu \mathrm{CT})$ is a modern non-invasive imaging modality which is used to generate three-dimensional high-resolution data sets of the in-vivo laboratory animals or excised organs. ${ }^{84,85}$ The volumetric $\mu \mathrm{CT}$ data are suitable for visualization and quantitative image analysis due to the isotropic voxel sizes and well-calibrated voxel units, especially of bone structures and calcifications. ${ }^{86}$ Various morphometric structural features have been reported in the literature to influence the augmentation ability of a specific bone type, including grafted bone particle sizes, porosity, biomechanical stress capacity, plasticity, and cortical bone content. ${ }^{18-20,87} \mu \mathrm{CT}$ has particularly added a deeper insight into evaluating calcified tissue and has been used in the quantitative analysis of bone formation in human and animal research to evaluate bone graft healing, in contrast to conventional histology which reveals more information on the cellular properties of the calcified tissue. ${ }^{18,38,88}$ In addition to bone mineral density, several morphometric parameters have been proposed to assess the mechanical characteristics of bone using three-dimensional $\mu \mathrm{CT}$ images. ${ }^{18,88,89}$ On the other hand, histological observation remains the gold standard in assessing hard tissue reactions. Histomorphometry is thus considered a valuable analytical method to quantify the changes in the mineralized bone and osteoid, and its surrounding environment, and provides unique information on cellularity and dynamic indices of bone remodelling. ${ }^{90}$ Various techniques and tissue staining methods have been developed for 
histological examination of the osteogenic potential of novel biomaterials used in bone grafting and assessing bone healing and regeneration.

\section{Interventional testing with composite Dentin/ß-TCP}

Composite bone grafting biomaterials consist of several micro and macro components mixed together with the aim to combine the favorable properties of each component. Testing various bone grafting materials in animal models requires reliable and predictable testing models to evaluate clinical interventions with respect to osteogenesis and healing. Critical size defects are the smallest size of skeletal tissue defect that will not completely heal in the animal's lifetime without an intervention. Calvarial critical-size defect models have been widely used in maxillofacial and orthopaedic research to test various interventions and novel bone grafting materials or modification of material preparations or surgical techniques. 38,90,91 Alveolar maxillary defects are unique defects with regard to their overlying soft tissues; the oral mucosa intraorally and the nasal mucosa as the nasal floor lining. Utilization of an animal model with a healed alveolar cleft defect, which mimics the oro-nasal communication in human patients with cleft lip and palate, would be the best model to test the healing pattern of bone graft materials and establish a proper anatomical structure. No previous research work has described dentin as a bone grafting substitute material in a reliable and widely accepted critical-size bone defect or in an alveolar maxillary cleft animal model. No comparative study has been conducted so far between dentin and injectable calcium phosphate bone substitute materials, which we believe may contribute positively to the enhancement of bone formation given its close chemical composition to both dentin and bone.

\section{Aims and Review of The Thesis}

The comprehensive objective of our studies was to methodically analyse various grafting materials being utilized in alveolar cleft bone grafting in order to improve the overall treatment outcomes of alveolar cleft repair in children with cleft lip and palate.

In our first paper, we aimed at systematically reviewing all up-to-date grafting options in humans, including autogenous grafts from different donor sites and the various regenerative bone substitute and tissue-engineered materials in the treatment of alveolar cleft defects.

In the second paper, we objectively investigated the morphometric micro-structure of the different bone grafts from commonly used donor sites in a human cadaveric study, and assessed their structural differences, hoping on shedding some light on their clinical behaviour during bone grafting. 
In the third paper, we explored the option of using human xenogenic dentin as grafting material in the rabbit tibia, and assessed its bone healing pattern.

In the fourth paper, we aimed at testing a composite xenognic dentin with ß-tricalcium phosphate as a grafting material using calvarial critical-size defects in rabbits for robust assessment of its bone healing and osteogenic potential.

In the fifth study, we aimed at developing a reliable alveolar cleft defect animal model in New Zealand White rabbits simulating the three-dimensional morphology in human patients with cleft lip and palate for interventional testing of novel bone substitute materials.

The aim of our last study was to evaluate bone regeneration potential and to quantify bone formation after grafting our experimental alveolar clefs defects in the rabbit model using in-situ formed composite xenogenic, non-demineralized dentin and B-TCP. We proposed adding dentin to the $ß$-TCP would enhance its bone regeneration potential and persistence of graft volume. 


\section{REFERENCES}

1. Grollemund B, Galliani E, Soupre V, Vazquez M, Guedeney A, Danion A. The impact of cleft lip and palate on the parent-child relationships. Archives de pediatrie: organe officiel de la Societe francaise de pediatrie. 2010;17:1380-5.

2. Cho-Lee G-Y, García-Díez E-M, Nunes R-A, Martí-Pagès C, Sieira-Gil R, Rivera-Baró A. Review of secondary alveolar cleft repair. Annals of maxillofacial surgery. 2013;3:46.

3. Allam E, Stone C, Windsor L. Cleft lip and palate: etiology, epidemiology, preventive and intervention strategies. Anat Physiol. 2014;4:1-6.

4. Wu C, Pan W, Feng C, Su Z, Duan Z, Zheng Q, et al. Grafting materials for alveolar cleft reconstruction: a systematic review and best-evidence synthesis. International Journal of Oral and Maxillofacial Surgery. 2017.

5. Craven C, Cole P, Hollier Jr L, Stal S. Ensuring success in alveolar bone grafting: a three-dimensional approach. Journal of Craniofacial Surgery. 2007;18:855-9.

6. Weissler EH, Paine KM, Ahmed MK, Taub PJ. Alveolar Bone Grafting and Cleft Lip and Palate: A Review. Plastic and reconstructive surgery. 2016;138:1287-95.

7. Lorot-Marchand A, Guerreschi P, Pellerin P, Martinot V, Gbaguidi C, Neiva C, et al. Frequency and socio-psychological impact of taunting in school-age patients with cleft lip-palate surgical repair. International journal of pediatric otorhinolaryngology. 2015;79:1041-8.

8. Stock NM, Stoneman K, Cunniffe C, Rumsey N. The Psychosocial Impact of Cleft Lip and/or Palate on Unaffected Siblings. The Cleft Palate-Craniofacial Journal. 2016;53:670-82.

9. Turner S, Rumsey N, Sandy J. Psychological aspects of cleft lip and palate. The European Journal of Orthodontics. 1998;20:407-15.

10. Eppley BL, Sadove AM. Management of alveolar cleft bone grafting —state of the art. The Cleft palate-craniofacial journal. 2000;37:229-33.

11. Horswell BB, Henderson JM. Secondary osteoplasty of the alveolar cleft defect. Journal of oral and maxillofacial surgery. 2003;61:1082-90.

12. Nauth A, Lane J, Watson JT, Giannoudis P. Bone Graft Substitution and Augmentation. Journal of Orthopaedic Trauma2015. p. S34-S8.

13. KolkA, Handschel J, Drescher W, Rothamel D, Kloss F, Blessmann M, et al. Current trends and future perspectives of bone substitute materials - from space holders to innovative biomaterials. Journal of cranio-maxillo-facial surgery : official publication of the European Association for Cranio-Maxillo-Facial Surgery. 2012;40:706-18.

14. Khojasteh A, Kheiri L, Motamedian SR, Nadjmi N. Regenerative medicine in the treatment of alveolar cleft defect: A systematic review of the literature. Journal of cranio-maxillo-facial surgery : official publication of the European Association for Cranio-Maxillo-Facial Surgery2015. p. 1608-13.

15. Janssen NG, Weijs WLJ, Koole R, Rosenberg AJWP, Meijer GJ. Tissue engineering strategies for alveolar cleft reconstruction: a systematic review of the literature. Clin Oral Investig: Springer Berlin Heidelberg; 2014. p. 219-26.

16. Fanuscu MI, Chang T-L. Three-dimensional morphometric analysis of human cadaver bone: microstructural data from maxilla and mandible. Clinical Oral Implants Research2004. p. 213-8.

17. Pothuaud L, Van Rietbergen B, Mosekilde L, Beuf O, Levitz P, Benhamou CL, et al. Combination of topological parameters and bone volume fraction better predicts the mechanical properties of trabecular bone. Journal of Biomechanics. 2002;35:1091-9.

18. Kim J-E, Shin J-M, Oh S-O, Yi W-J, Heo M-S, Lee S-S, et al. The three-dimensional microstructure of trabecular bone: analysis of site-specific variation in the human jaw bone. Imaging science in dentistry. 2013;43:227-33. 
19. Kolk A, Handschel J, Drescher W, Rothamel D, Kloss F, Blessmann M, et al. Current trends and future perspectives of bone substitute materials - from space holders to innovative biomaterials. J Craniomaxillofac Surg2012. p. 706-18.

20. Kon K, Shiota M, Ozeki M, Yamashita Y, Kasugai S. Bone augmentation ability of autogenous bone graft particles with different sizes: a histological and micro-computed tomography study. Clinical Oral Implants Research: Blackwell Publishing Ltd; 2009. p. 1240-6.

21. Vinci R, Rebaudi A, Capparè P, Gherlone E. Microcomputed and histologic evaluation of calvarial bone grafts: a pilot study in humans. International Journal of Periodontics and Restorative Dentistry. 2011;31:389.

22. Ozaki W, Buchman SR. Volume maintenance of onlay bone grafts in the craniofacial skeleton: micro-architecture versus embryologic origin. Plastic and reconstructive surgery. 1998;102:291-9.

23. Phillips JH, Rahn BA. Fixation effects on membranous and endochondral onlay bone graft revascularization and bone deposition. Plastic and reconstructive surgery. 1990;85:891-7.

24. Ma'amon AR, Telfah $\mathrm{H}$. Secondary alveolar bone grafting: the dilemma of donor site selection and morbidity. British Journal of Oral and Maxillofacial Surgery. 2008;46:665-70.

25. Seifeldin SA. Is alveolar cleft reconstruction still controversial? (Review of literature). Saudi Dent J2016. p. 3-11.

26. Nkenke E, Neukam FW. Autogenous bone harvesting and grafting in advanced jaw resorption: morbidity, resorption and implant survival. Eur J Oral Implantol. 2014;7:S203-17.

27. Sakkas A, Wilde F, Heufelder M, Winter K, Schramm A. Autogenous bone grafts in oral implantology-is it still a "gold standard"? A consecutive review of 279 patients with 456 clinical procedures. International Journal of Implant Dentistry. 2017;3:23.

28. Chung VH, Chen AY, Jeng LB, Kwan CC, Cheng SH, Chang SC. Engineered autologous bone marrow mesenchymal stem cells: alternative to cleft alveolar bone graft surgery. The Journal of craniofacial surgery. 2012;23:1558-63.

29. Jensen AT, Jensen SS, Worsaae N. Complications related to bone augmentation procedures of localized defects in the alveolar ridge. A retrospective clinical study. Oral and maxillofacial surgery. 2016;20:115-22.

30. Gładysz D, Hozyasz KK. Stem cell regenerative therapy in alveolar cleft reconstruction. Arch Oral Biol2015. p. 1517-32.

31. Kawata T, Kohno S, Fujita T, Sugiyama H, Tokimasa C, Kaku M, et al. New biomaterials and methods for craniofacial bone defect: chondroid bone grafts in maxillary alveolar clefts. Journal of craniofacial genetics and developmental biology. 2000;20:49-52.

32. Kim YK, Lee J, Yun JY, Yun PY, Um IW. Comparison of autogenous tooth bone graft and synthetic bone graft materials used for bone resorption around implants after crestal approach sinus lifting: a retrospective study. Journal of periodontal \& implant science. 2014;44:216-21.

33. Pilanci O, Cinar C, Kuvat SV, Altintas M, Guzel Z, Kilic A. Effects of Hydroxyapatite on Bone Graft Resorption in an Experimental Model of Maxillary Alveolar Arch Defects. Archives of Clinical and Experimental Surgery (ACES). 2013;2:170-5.

34. Clokie CM, Moghadam H, Jackson MT, Sandor GK. Closure of critical sized defects with allogenic and alloplastic bone substitutes. Journal of Craniofacial Surgery. 2002;13:111-21.

35. Haddad AJ, Peel SA, Clokie CM, Sándor GK. Closure of rabbit calvarial critical-sized defects using protective composite allogeneic and alloplastic bone substitutes. Journal of Craniofacial Surgery. 2006;17:926-34.

36. Su-Gwan K, Hak-Kyun K, Sung-Chul L. Combined implantation of particulate dentine, plaster of Paris, and a bone xenograft (Bio-Oss $®$ ) for bone regeneration in rats. Journal of Cranio-Maxillofacial Surgery. 2001;29:2828.

37. Zhou AJ-J, Peel SA, Clokie CM. An evaluation of hydroxyapatite and biphasic calcium phosphate in combination with Pluronic F127 and BMP on bone repair. Journal of Craniofacial Surgery. 2007;18:1264-75. 
38. Lappalainen O-P, Karhula SS, Haapea M, Kauppinen S, Finnilä M, Saarakkala S, et al. Micro-CT Analysis of Bone Healing in Rabbit Calvarial Critical-Sized Defects with Solid Bioactive Glass, Tricalcium Phosphate Granules or Autogenous Bone. Journal of oral \& maxillofacial research. 2016;7.

39. Saikia K, Bhattacharya T, Bhuyan S, Talukdar D, Saikia S, Jitesh P. Calcium phosphate ceramics as bone graft substitutes in filling bone tumor defects. Indian journal of orthopaedics. 2008;42:169.

40. Lappalainen O-P, Karhula S, Haapea M, Kyllönen L, Haimi S, Miettinen S, et al. Bone healing in rabbit calvarial critical-sized defects filled with stem cells and growth factors combined with granular or solid scaffolds. Child's Nervous System. 2016;32:681-8.

41. Sharif F, Rehman IU, Muhammad N, MacNeil S. Dental materials for cleft palate repair. Materials Science and Engineering: C. 2016;61:1018-28.

42. Janssen NG, Weijs WL, Koole R, Rosenberg AJ, Meijer GJ. Tissue engineering strategies for alveolar cleft reconstruction: a systematic review of the literature. Clinical oral investigations. 2014;18:219-26.

43. Janssen NG, de Ruiter AP, van Hout WM, van Miegem V, Gawlitta D, Groot FB-d, et al. Microstructured $\beta$-Tricalcium Phosphate Putty Versus Autologous Bone for Repair of Alveolar Clefts in a Goat Model. The Cleft Palate-Craniofacial Journal. 2016.

44. Bittermann GKP, Janssen NG, van Leeuwen M, van Es RJ. One-Year Volume Stability of Human Facial Defects Filled With a $\beta$-Tricalcium Phosphate-Hydroxyl Apatite Mixture (Atlantik). Journal of Craniofacial Surgery. 2014;25:372-4.

45. Andersson L. Dentoalveolar ankylosis and associated root resorption in replanted teeth. Experimental and clinical studies in monkeys and man. Swedish dental journal Supplement. 1988;56:1-75.

46. Al-Asfour A, Andersson L, Kamal M, Joseph B. New bone formation around xenogenic dentin grafts to rabbit tibia marrow. Dental traumatology : official publication of International Association for Dental Traumatology. 2013;29:455-60.

47. Maslamani M, Almusawi A, Joseph B, Gabato S, Andersson L. An experimental model for studies on delayed tooth replantation and ankylosis in rabbits. Dental Traumatology. 2016;32:443-9.

48. Andersson L, Blomlöf L, Lindskog S, Feiglin B, Hammarström L. Tooth ankylosis: clinical, radiographic and histological assessments. International journal of oral surgery. 1984;13:423-31.

49. Andersson L, Bodin I, Sörensen S. Progression of root resorption following replantation of human teeth after extended extraoral storage. Dental Traumatology. 1989;5:38-47.

50. Trope M. Avulsion of permanent teeth: theory to practice. Dental Traumatology. 2011;27:281-94.

51. Al-Asfour A, Farzad P, Andersson L, Joseph B, Dahlin C. Host tissue reactions of non-demineralized autogenic and xenogenic dentin blocks implanted in a non-osteogenic environment. An experimental study in rabbits. Dental traumatology : official publication of International Association for Dental Traumatology. 2014;30:198203.

52. Andersson L. Dentin xenografts to experimental bone defects in rabbit tibia are ankylosed and undergo osseous replacement. Dental traumatology : official publication of International Association for Dental Traumatology. 2010;26:398-402.

53. Andersson L, Ramzi A, Joseph B. Studies on dentin grafts to bone defects in rabbit tibia and mandible; development of an experimental model. Dental traumatology : official publication of International Association for Dental Traumatology. 2009;25:78-83.

54. Malmgren B. Ridge preservation/decoronation. Journal of endodontics. 2013;39:S67-S72.

55. Malmgren B, Tsilingaridis G, Malmgren O. Long-term follow up of 103 ankylosed permanent incisors surgically treated with decoronation-a retrospective cohort study. Dental Traumatology. 2015;31:184-9.

56. Al-Asfour A, Farzad P, Al-Musawi A, Dahlin C, Andersson L. Demineralized Xenogenic Dentin and Autogenous Bone as Onlay Grafts to Rabbit Tibia. Implant dentistry. 2017;26:232-7. 
57. Fugazzotto P, De Paoli S, Benfenati S. The use of allogenic freeze-dried dentin in the repair of periodontal osseous defects in humans. Quintessence international (Berlin, Germany: 1985). 1986;17:461.

58. Gomes MF, dos Anjos MJ, Nogueira TO, Guimaraes SA. Histologic evaluation of the osteoinductive property of autogenous demineralized dentin matrix on surgical bone defects in rabbit skulls using human amniotic membrane for guided bone regeneration. The International journal of oral \& maxillofacial implants. 2001;16:563-71.

59. Moharamzadeh K, Freeman C, Blackwood K. Processed bovine dentine as a bone substitute. British Journal of Oral and Maxillofacial Surgery. 2008;46:110-3.

60. Kim YK, Lee J, Um IW, Kim KW, Murata M, Akazawa T, et al. Tooth-derived bone graft material. Journal of the Korean Association of Oral and Maxillofacial Surgeons. 2013;39:103-11.

61. Harling TR, Stelnicki EJ, Hedrick MH, Longaker MT. In utero models of craniofacial surgery. World journal of surgery. 2003;27:108-16.

62. Hedrick MH, Rice HE, Vander Wall KJ, Adzick NS, Harrison MR, Siebert J, et al. Delayed in utero repair of surgically created fetal cleft lip and palate. Plastic and reconstructive surgery. 1996;97:900-5.

63. Papadopoulos MA, Papadopulos NA, Jannowitz C, Boettcher P, Henke J, Stolla R, et al. Three-dimensional cephalometric evaluation of maxillary growth following in utero repair of cleft lip and alveolar-like defects in the mid-gestational sheep model. Fetal diagnosis and therapy. 2006;21:105-14

64. Wenghoefer MHO, Deprest J, Goetz W, Kuijpers-Jagtman AM, Bergé S. Prenatal cleft lip and maxillary alveolar defect repair in a 2-step fetal lamb model. Journal of Oral and Maxillofacial Surgery. 2007;65:2479-86.

65. Caballero M, Morse JC, Halevi AE, Emodi O, Pharaon MR, Wood JS, et al. Juvenile Swine Surgical Alveolar Cleft Model to Test Novel Autologous Stem Cell Therapies. Tissue Eng Part C Methods: Mary Ann Liebert, Inc. 140 Huguenot Street, 3rd Floor New Rochelle, NY 10801 USA; 2015. p. 898-908.

66. de Ruiter A, Meijer G, Dormaar T, Janssen N, van der Bilt A, Slootweg P, et al. $\beta$-TCP versus autologous bone for repair of alveolar clefts in a goat model. Cleft Palate Craniofac J: Allen Press Publishing Services; 2011. p. 654-62.

67. el-Bokle D, Smith SJ, Germane N, Sharawy M. New technique for creating permanent experimental alveolar clefts in a rabbit model. Cleft Palate Craniofac J1993. p. 542-7.

68. El-Deeb M, Horswell B, Waite DE. A primate model for producing experimental alveolar cleft defects. J Oral Maxillofac Surg1985. p. 523-7.

69. Gritli-Linde A. The mouse as a developmental model for cleft lip and palate research. Front Oral Biol: S. KARGER AG; 2012. p. 32-51.

70. Ishii Y. [Experimental study of secondary bone graft of alveolar clefts using bone morphogenetic protein (BMP)]. Kokubyo Gakkai zasshi The Journal of the Stomatological Society, Japan. 2001;68:111-24.

71. Liang L, Liu C. Trans-sutural distraction osteogenesis for alveolar cleft repair: an experimental canine study. Cleft Palate Craniofac J: Allen Press Publishing Services; 2012. p. 701-7.

72. Liao LS, Tan Z, Zheng Q, Wu J, Shi B, He X, et al. Animal experimental study on repairing alveolar clefts by using rectilinear distraction osteogenesis. Journal of plastic, reconstructive \& aesthetic surgery : JPRAS. 2009;62:1573-9

73. Mostafa NZ, Doschak MR, Major PW, Talwar R. Reliable critical sized defect rodent model for cleft palate research. Journal of cranio-maxillo-facial surgery : official publication of the European Association for Cranio-Maxillo-Facial Surgery2014. p. 1840-6.

74. Nguyen PD, Lin CD, Allori AC, Ricci JL, Saadeh PB, Warren SM. Establishment of a critical-sized alveolar defect in the rat: a model for human gingivoperiosteoplasty. Plastic and reconstructive surgery. 2009;123:817-25.

75. Raposo-Amaral CE, Kobayashi GS, Almeida AB, Bueno DF, Freitas FRdSe, Vulcano LC, et al. Alveolar osseous defect in rat for cell therapy: preliminary report. Acta Cir Bras2010. p. 313-7. 
76. Sawada Y, Hokugo A, Nishiura A, Hokugo R, Matsumoto N, Morita S, et al. A trial of alveolar cleft bone regeneration by controlled release of bone morphogenetic protein: an experimental study in rabbits. Oral surgery, oral medicine, oral pathology, oral radiology, and endodontics. 2009;108:812-20.

77. Wu L-I, Zhao Y, Chen C. [Establishment of the animal model with unilateral alveolar cleft and its effect on the nose growth]. Zhonghua Zheng Xing Wai Ke Za Zhi2010. p. 39-42.

78. Xu Y, Sun J, Chen Z. Establishment of a rat model for alveolar cleft with bone wax. Journal of oral and maxillofacial surgery : official journal of the American Association of Oral and Maxillofacial Surgeons. 2015;73:733. e1-10.

79. Kim J-H, Moon H-J, Kim T-H, Jo J-M, Yang SH, Naskar D, et al. A novel in vivo platform for studying alveolar bone regeneration in rat. Journal of tissue engineering. 2013;4:2041731413517705.

80. Li Y, Chen S-K, Li L, Qin L, Wang X-L, Lai Y-X. Bone defect animal models for testing efficacy of bone substitute biomaterials. Journal of Orthopaedic Translation. 2015;3:95-104.

81. Mapara M, Thomas BS, Bhat K. Rabbit as an animal model for experimental research. Dental research journal. 2012;9.

82. Pearce A, Richards R, Milz S, Schneider E, Pearce S. Animal models for implant biomaterial research in bone: a review. Eur Cell Mater. 2007;13:1-10.

83. Wang X, Mabrey JD, Agrawal CM. An interspecies comparison of bone fracture properties. Biomedical Materials and Engineering. 1998;8:1-10.

84. Ehling J, Babickova J, Gremse F, Klinkhammer BM, Baetke S, Knuechel R, et al. Quantitative Micro-Computed Tomography Imaging of Vascular Dysfunction in Progressive Kidney Diseases. Journal of the American Society of Nephrology: JASN. 2016;27:520-32.

85. Gremse F, Theek B, Kunjachan S, Lederle W, Pardo A, Barth S, et al. Absorption reconstruction improves biodistribution assessment of fluorescent nanoprobes using hybrid fluorescence-mediated tomography. Theranostics. 2014;4:960-71.

86. Gremse F, Stark M, Ehling J, Menzel JR, Lammers T, Kiessling F. Imalytics Preclinical: Interactive Analysis of Biomedical Volume Data. Theranostics. 2016;6:328-41.

87. Katranji A, Misch K, Wang H-L. Cortical bone thickness in dentate and edentulous human cadavers. Journal of periodontology. 2007;78:874-8.

88. Ulrich D, Van Rietbergen B, Laib A, Ruegsegger P. The ability of three-dimensional structural indices to reflect mechanical aspects of trabecular bone. Bone. 1999;25:55-60.

89. Hildebrand T, Laib A, Müller R, Dequeker J, Rüegsegger P. Direct three-dimensional morphometric analysis of human cancellous bone: microstructural data from spine, femur, iliac crest, and calcaneus. J Bone Miner Res: John Wiley and Sons and The American Society for Bone and Mineral Research (ASBMR); 1999. p. 1167-74.

90. Parfitt AM, Drezner MK, Glorieux FH, Kanis JA, Malluche H, Meunier PJ, et al. Bone histomorphometry: standardization of nomenclature, symbols, and units: report of the ASBMR Histomorphometry Nomenclature Committee. Journal of bone and mineral research. 1987;2:595-610.

91. Hussain I, Moharamzadeh K, Brook IM, Jose de Oliveira Neto P, Salata LA. Evaluation of osteoconductive and osteogenic potential of a dentin-based bone substitute using a calvarial defect model. Int J Dent. 2012;2012:396316. 


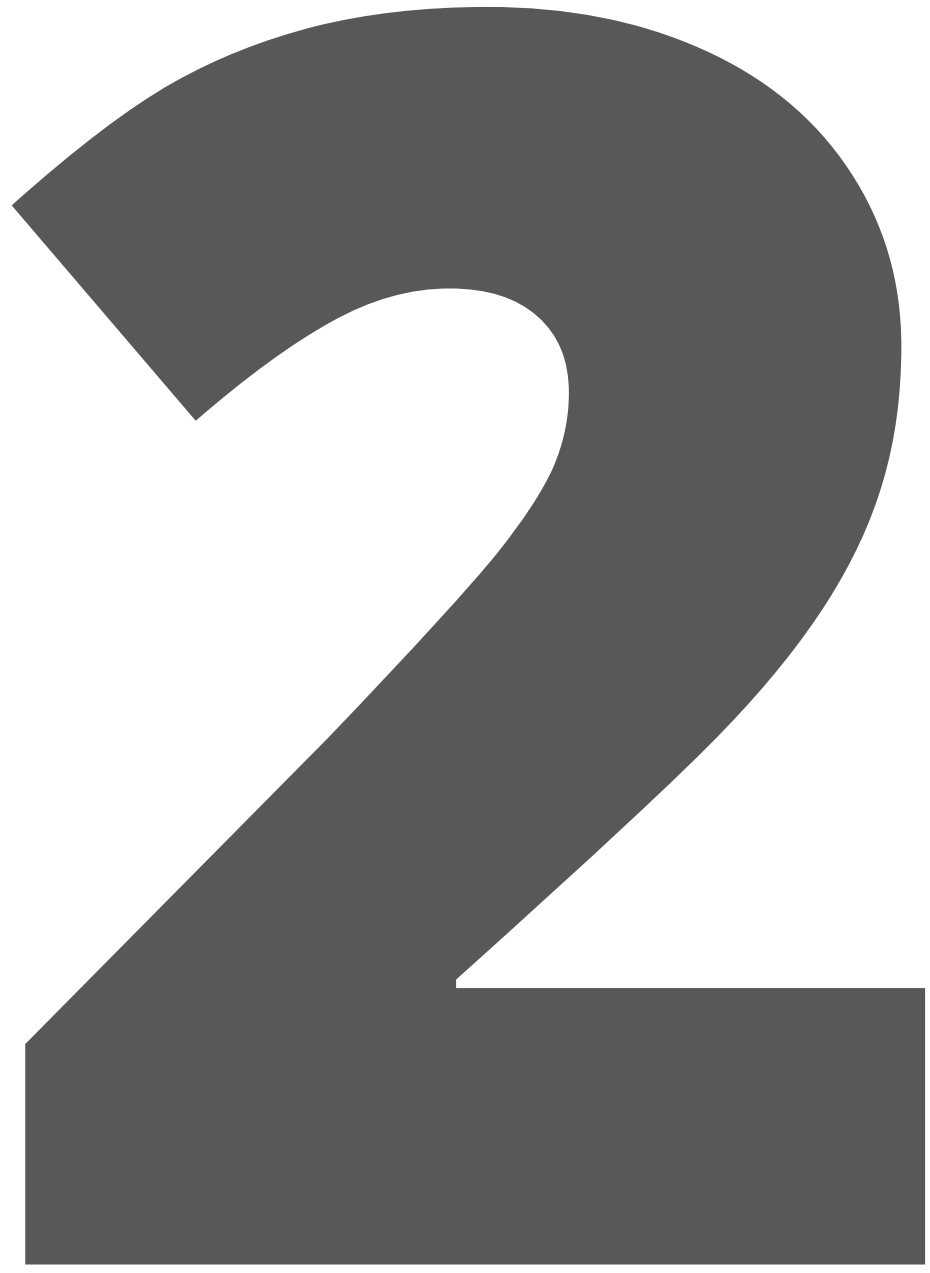




\section{"Volumetric Comparison of Autogenous Bone and Tissue-Engineered Bone Replacement Materials in Alveolar Cleft Repair: A Systematic Review and Meta-Analysis"}

Mohammad Kamal; Ali H. Ziyab; Alexander Bartella; David Mitchell; Adel Al-Asfour; Frank Hölzle; Peter Kessler; Bernd Lethaus

Published in the British Journal of Oral and Maxillofacial Surgery. 2018 July; 56(6):453-462. doi: 10.1016/j.bjoms.2018.05.007 


\section{ABSTRACT}

Objectives: The goal of alveolar cleft reconstruction in patients with cleft lip and palate is to improve tissue quality, structural stability, and bone volume. This study is a systematic review with meta-analysis of volumetric bone fill using autogenous bone and various tissue-engineered bone substitutes.

Materials and Methods: Electronic search on MEDLINE, EMBASE, SCOPUS, Web of Science, and grey literature was conducted according to the Preferred Reporting Items for Systematic Reviews and Meta-Analyses (PRISMA) guidelines. Studies with volumetrically reported grafting outcome were included in the meta-analysis.

Results: Out of 1276 studies, 26 were included in the meta-analysis. Pooled analysis of 25 studies using autogenous bone revealed a statistically significant reduction in cleft volume equivalent to $62.0 \%$ bone fill $(95 \% \mathrm{Cl}: 54.3,69.6)$, in contrast to 10 studies using a tissue-engineered material with percentage bone filling of $68.7 \%$ (95\% Cl: $54.5,82.8)$. The estimated pooled effect sizes across studies showed that there is no statistically significant difference between the two major intervention groups ( $P$-value $=0.901)$.

Conclusions: Our statistical analysis show no significant difference between autogenous bone grafts and novel tissue-engineered materials with regard to cleft filling capability.

Systematic review registration: International Prospective Register of Systematic Reviews, PROSPERO (CRD42017065045). 


\section{INTRODUCTION}

Cleft lip and palate is a malformation caused by the incomplete fusion of facial prominences during embryonic development. This results in a bony defect in the maxillary process and distruption of teeth eruption. Skeletal grafting of alveolar cleft deformities is an essential step to re-establish the bony continuity of the alveolar arch and to create a favorable anatomy for dental rehabilitation. ${ }^{1-3}$

Reconstruction of these defects is done via bone grafting procedures using autogenous bone, along with various tissue-engineered bone replacement materials. ${ }^{4-9}$ Autologous bone can be obtained from several donor sites including the iliac crest, calvarium, mandibular symphysis, mandibular ramus, and the tibia. Nonetheless, each donor site carries its unique risks and morbidities. 3, 10

A current review of the literature revealed the utilization of multiple bone substitute materials for bone regeneration. ${ }^{5-9,11}$ These materials can be categorized into different groups (Table 1) based on their composition which includes:

1. Growth Factors:

Biological factors based on molecular cellular interactions leading to enhanced osteogensis. They include Bone Morphogenic Proteins (BMP), Platelet Rich Plasma (PRP), and Platelet Derived Growth Factors (PDGF).

2. Enhanced Scaffolds and Cell Therapy (MSC, OB)/Growth Factors combination:

Multiple scaffolds which are utilized to promote osteoconduction. Scaffolds are usually impregnated with cellular components either with Mesenchymal Stem Cells (MSC) or Osteoblasts $(\mathrm{OB})$, which can also be treated with growth factor to further enhance bone formation.

3. Biocomposites and Hemostatic Agents:

Biocompatible synthetic materials or biological components that promote osteoconduction and enhance the formation of blood coagulum, leading to enhanced delivery of growth factors and promotion of osteogenesis. Biocompatible alloplastic materials include Calcium Phosphate and Hydroxyapatite, whereas biologic hemostatic agents include Fibrin glue and similar hemostatic agents.

Assessment of bone grafting treatment outcome was performed mostly through the Bergland grading system using two-dimensional (2D) radiographs including panoramic, occlusal, and periapical films to measure interalveolar bone height. ${ }^{12,13}$ Nevertheless, the reliability of 2D image quality for clinical assessment is questionable due to several drawbacks, including the lack of volumetric data, distortion and magnification issues during 
image capture, an overlap of anatomical structures, and the absence of a reliable set of identifiable anatomical landmarks. ${ }^{13,14}$ With the recent advancement in medical imaging using computed tomography and the ease of volumetric reconstruction for computational analysis, the use of 3D volumetric analysis is becoming more frequent in assessing bone grafting treatment outcome. ${ }^{13,15}$

Table 1 Groups of grafting materials.

Autogenous Bone Grafts
1. Mlac Crest Bone Graft
2. Mandibular Symphysis Bone Graft
3. Cranial Bone Graft
4. Tibial Bone Graft

\section{Tissue-Engineered Bone Replacement Materials}

1. Growth Factors (BMP, PRP, PDGF)

2. Enhanced Scaffolds and Cell Therapy (MSC, OB)/Growth Factors combination

3. Biocomposites and Hemostatic Agents (Fibrin-glue, Calcium Phosphate, HydroxyApetite)

BMP: Bone Morphogenic Protein; PRP: Platelet Rich Plasma; PDGF: Platelet Derived Growth Factor; MSC: Mesenchymal Stem Cell; OB: Osteoblasts

Thus far, two major factors have contributed to the heterogenity in assessing treatment outcomes, namely the grafting material used (i.e., autogenous versus tissue-engineered) and the method of measuring the cleft volume pre- and post-operatively. Current literature lacks a comprehensive study comparing outcomes (e.g., percentage bone volume fill) of different interventions based on 3D volumetric measurements. Hence, this systematic review and meta-analysis sought to provide insights on the effectiveness of the two major treatment approaches (autogenous bone from various donor sites and the different tissue-engineering materials). The results of this meta-analysis provide evidence whether treatment with regenerative bone substitutes yields improved outcomes compared to treatment with autogenous bone grafts. 


\section{MATERIALS AND METHODS}

\section{Study Protocol and Registration:}

The present study was conducted according to the Preferred Reporting Items for Systematic Reviews and Meta-Analyses (PRISMA) guidelines. ${ }^{16}$ A protocol was established according to the evidence-based PICO model (population, intervention, comparison and outcome) to answer the following question:

"In patients with congenital cleft lip and palate with alveolar cleft defect, what is the effectiveness of different grafting surgical interventions (autogenous or tissue-engineered bone substitutes) in filling the alveolar cleft defect with bone as detected by radiographic volumetric imaging modality?"

The protocol was reviewed among all authors and subsequently registered in the International Prospective Register of Systematic Reviews, PROSPERO (Registration CRD42017065045) and can be accessed at: https://www.crd.york.ac.uk/PROSPERO/display_record.asp?ID=CRD42017065045.

\section{Information Resources and Search Strategy:}

A comprehensive search of major online electronic databases, including MEDLINE, EMBASE, SCOPUS, and WEB OF SCIENCE, was performed. The search covered published literature in each database from its inception to February 1st, 2017. No language restriction was applied. Searching relevant terms and keywords included "cleft lip and palate", "bone grafting", "maxillary alveolus", and "transplantation" (Table S1). Grey literature search and cross references were also performed.

\section{Eligibility criteria:}

We included all interventional studies, conducted in humans, assessing the effectiveness of treatment of alveolar cleft defects with various bone grafting techniques in patients with cleft lip and palate. All epidemiological study designs that allow evaluating the effects of interventions were included, such as randomized or quasi-randomized controlled/ uncontrolled trials, uncontrolled longitudinal case series, and before-and-after studies. Table 2 presents the inclusion and exclusion criteria in details. The included studies should have reported pre-operative and post-operative cleft volume using volumetric imaging (cc, ml, mm3, cm3), and/or reported percentage bone filling (residual bone defect, bone formation, residual cleft), which would allow us to volumetrically compare the results of the various grafting materials. 
Table S1 The electronic databases searched and search strategy as of 01.02.2017.

\begin{tabular}{|c|c|c|}
\hline Electronic database & Search strategy & $\begin{array}{l}\text { No. of } \\
\text { hits per } \\
\text { database }\end{array}$ \\
\hline $\begin{array}{l}\text { EMBASE } \\
\text { start - 01.02.2017 }\end{array}$ & $\begin{array}{l}\text { ((cleft lip) OR (cleft palate) OR Harelip OR "Cleft Lip" OR "Cleft Palate") } \\
\text { AND } \\
\text { (alveolar OR (alveolus cleft) OR (alveolar cleft)) AND } \\
\text { ((bone grafting) OR (bone graft) OR "Bone Transplantation" OR (Bone } \\
\text { Transplantation) OR } \\
\text { "Alveolar Bone Grafting") }\end{array}$ & 874 \\
\hline $\begin{array}{l}\text { MEDLINE (Pubmed) } \\
\text { start - 01.02.2017 }\end{array}$ & $\begin{array}{l}\text { ((((((((((cleft lip) OR cleft palate) OR Harelip) OR “Cleft Lip"[Mesh]) } \\
\text { OR "Cleft Palate"[Mesh])) AND alveolar)) OR alveolus cleft) OR } \\
\text { alveolar cleft)) AND (((((bone grafting) OR bone graft) OR “Bone } \\
\text { Transplantation"[Mesh]) OR Bone Transplantation) OR "Alveolar Bone } \\
\text { Grafting”[Mesh]) }\end{array}$ & 897 \\
\hline $\begin{array}{l}\text { Scopus } \\
\text { start - 01.02.2017 }\end{array}$ & $\begin{array}{l}\text { (TITLE-ABS-KEY(cleft lip) OR TITLE-ABS-KEY(cleft palate) OR TITLE-ABS- } \\
\text { KEY(Harelip) OR TITLE-ABS-KEY("cleft lip") OR TITLE-ABS-KEY(“cleft } \\
\text { palate")) AND (TITLE-ABS-KEY(alveolar) OR TITLE-ABS-KEY(alveolus } \\
\text { cleft) OR TITLE-ABS-KEY(alveolar cleft)) AND (TITLE-ABS-KEY(bone } \\
\text { grafting) OR TITLE-ABS-KEY(bone graft) OR TITLE-ABS-KEY("bone } \\
\text { transplantation") OR TITLE-ABS-KEY(bone transplantation) OR TITLE- } \\
\text { ABS-KEY("alveolar bone grafting")) }\end{array}$ & 980 \\
\hline $\begin{array}{l}\text { ISI Web of Knowledge } \\
\text { start - 01.02.2017 }\end{array}$ & $\begin{array}{l}\text { (TS=(cleft lip) OR TS=(cleft palate) OR TS=(Harelip) OR TS=("cleft lip") } \\
\text { OR TS=("cleft palate")) AND (TS=(alveolar) OR TS=(alveolus cleft) } \\
\text { OR TS=(alveolar cleft)) AND (TS=(bone grafting) OR TS=(bone graft) } \\
\text { OR TS=("bone transplantation") OR TS=(bone transplantation) OR } \\
\text { TS=("alveolar bone grafting")) }\end{array}$ & 910 \\
\hline
\end{tabular}

\section{Study selection and data extraction:}

In the screening round, two reviewers (M.K. and A.B.) independently performed the selection process of the articles based on the title and abstract and studies were assigned as "include", "exclude", and "maybe". Any disagreement was resolved by a third review (B.L.). Next, full-text of articles assigned as "include" and "maybe" were obtained for a second round of reviewing. Assessment of the full-text articles in the second round and defining the modality of intervention was performed by the same two reviewers (M.K. and A.B.). Disagreement was again resolved by a third review (B.L.).

From each eligible study, two authors (M.K. and A.H.Z.) independently extracted the relevant information. Disagreements were resolved by discussion. The following data were obtained from each study using a structured data extraction table: name of first author, year of publication, study design, type of intervention(s), sample size (if multiple intervention arms were used, then sample size was reported accordingly), cleft type, average age of study participants, imaging modality, method of volumetric analysis, length of follow-up, mean of pre- and post-operative cleft volume and measure of dispersion (e.g., standard deviation, range, and/or variance), and mean percentage bone filling (residual bone defect, 
bone formation, residual cleft). In instances where summary statistics were not available and raw data were provided within the publication, authors calculated the needed summary measures (e.g., mean and standard deviation).

Table 2 Inclusion and exclusion criteria.
Inclusion
1. Studies in humans of all ages
2. Patients with the diagnosis of unilateral/bilateral cleft lip and/or palate involving the alveolar process
3. At least one study arm with surgical alveolar bone grafting as an Intervention
4. Randomized or quasi- randomized controlled trials, controlled clinical trials, prospective studies, and retrospective studies
5. Volumetric imaging obtained for postoperative assessment (Computed Tomography, Cone Beam Computed Tomography)
6. Reported perioperative and postoperative cleft volume using volumetric imaging (cc, ml, mm3, cm3), or reported percentage bone filling (residual bone defect, bone formation, residual cleft)
7. Postoperative volumetric assessment performed minimum 3 months postoperatively

\section{Exclusion}

1. Trials in animals

2. Non-clinical studies and all other research types (i.e. editorials, textbooks, technical reports, etc)

3. Case reports

4. No volumetric imaging values

\section{Assessment of study quality and risk of bias}

A qualitative rating methodology previously described by Al-Moraissi et al was utilized by combining the proposed quality assessment criteria from the MOOSE (Meta-Analysis of Observational Studies in Epidemiology) statement, STROBE (Strengthening the Reporting of Observational Studies in Epidemiology) statement, and PRISMA statement to evaluate the quality of the scientific evidence of the studies. ${ }^{17-20}$

Risk of potential bias for each study was classified after assessing the following 5 criteria: randomization in allocation, definition of the criteria for inclusion and exclusion into the study, reporting of loss to follow-up, utilization of validated measurements, and description of statistical analysis. A study was considered to have a low risk of bias when all of the 5 parameters were present in the study, while it was considered a moderate risk when 4 parameters were fulfilled, and high risk of bias was defined by studies having met 3 or less of these criteria. ${ }^{17}$

\section{Statistical analysis}

Since the outcome measure is continuous (cleft volume) and we are interested in determining if the different treatment approaches performed differentially in reducing the cleft volume, the standardized mean difference (SMD), using the Hedges' g statistic, was estimated and considered as the measure of effect size. ${ }^{21}$ The Hedges' $g$ statistic estimates SMDs by dividing the difference in means by pooled standard deviations. In general, SMD 
is applied when the same outcome variable is measured using different scales and/or different measuring instruments with an aim to standardize the results of the different studies to a uniform unit-free scale. In the current study, the SMD was calculated as the difference between pre- and post-operative cleft volume; thus, a negative SMD value represents a decrease in the cleft volume. Although SMD is difficult to interpret, rules of thumb exist for interpreting SMDs; for example, a SMD $<0.40$ is considered a small effect, 0.40 to 0.70 moderate, and $>0.70$ is a large effect. ${ }^{22}$ In addition to estimating SMD, average percentage bone filling was estimated as follows: \% bone fill = [(Pre-operation cleft volume - Post-operation cleft volume) / Pre-operation cleft volume] x 100.

Meta-analyses were performed to calculate pooled (overall) SMDs and 95\% confidence intervals (Cls) across studies using random effects model in the presence of heterogeneity ( $\left.R^{2}>50 \%\right) ;$ otherwise, fixed effects model was applied. ${ }^{23}$ Specifically, meta-analyses were conducted to estimate a pooled SMD for studies using autogenous bone grafts and studies using tissue-engineered bone replacement material separately. Moreover, subgroup analyses were performed within each of the two major treatment groups. Q-test for heterogeneity based on random-effects model was used to test whether SMDs vary across subgroups. If considerable heterogeneity $\left(R^{2}>50 \%\right)$ was detected, additional subgroup analyses were performed to investigate potential reasons based on previously described methodological risk of bias assessment. Publication bias was visually examined by using funnel plots. Analyses were preformed using the Comprehensive Meta-Analysis software version 3.3.070 (Biostat, Inc., Englewood, NJ, USA).

Briefly, the Q-test for heterogeneity examines the null hypothesis of homogeneity that all studies (or subgroups) included in the meta-analysis are evaluating the same effect; hence, a pre-determined statistical significance level (e.g., $a=0.05$ ) is used to reject the null hypothesis of homogeneity. On the other hand, $l^{2}$ quantifies heterogeneity by describing the percentage of variability across studies that is attributable to heterogeneity rather than chance. ${ }^{23} R$ takes values between $0 \%$ and $100 \%$, with $0 \%$ indicating no observed heterogeneity and larger values indicate increasing heterogeneity across studies. In the current report, $R$ was used to aid choosing between applying fixed effect $(R \leq 50 \%)$ or random effects ( $R>50 \%$ ) model. Whereas, Q-test for heterogeneity was used to test whether SMDs vary across different intervention subgroups. 


\section{RESULTS}

\section{Search results and characteristics of the studies}

A total of 3661 articles were identified by the initial search of electronic databases and 5 additional publications were identified from the grey literature (Figure 1). After removing duplicates, 1276 titles and abstracts were subjected to screening. We excluded 1082 articles that did not meet the inclusion criteria. Full-text of the remaining 198 articles were further assessed for eligibility, of which 32 articles were eligible for inclusion in the study. After further excluding 6 studies that reported data from the same cohorts and/or studies that did not provide sufficient information, 26 studies were included in the current systematic review and meta-analysis. ${ }^{24-49}$

Methodological and detailed characteristics of the selected studies are shown in table S2. Of the 26 studies included in the meta-analysis, 6 were randomized, 9 prospective and 11 retrospective studies. The time point of postoperative radiographic assessment varied among the studies and ranged from 3 to 36 months. When multiple time points were reported, the farthest from the point of intervention was used. In studies with more than 1 intervention group, each comparison group was considered independently for the meta-analysis. Autogenous bone was used in 25 studies; 22 conventional iliac crest bone grafts, 1 calvarial, 1 tibial, and 1 mandibular symphysis grafts. Tissue-engineered bone substitutes were used in 10 studies, of which 6 were Growth Factors, 2 were Enhanced Scaffolds and Cell Therapy, and 2 were Biocomposites and Hemostatic Agents.

\section{Risk of Bias in Individual Studies}

Quality assessment of the included studies according to the aforementioned instrumental method revealed 6 studies with a low risk of bias, 9 with moderate risk of bias, and 11 with high risk of bias (Table S3).

\section{Meta-analysis findings}

Pooled analysis of 25 studies (Table 3) that used autogenous grafts as the intervention revealed statistically significant large reduction in cleft volume (overall SMD $=-1.91,95 \%$ $\mathrm{Cl}$ : $-2.25,-1.57, P$-value $<0.001,12=77.3 \%$; Figure 2$)$. This reduction is equivalent to, on average, a $62.0 \%$ (95\% Cl: 54.3, 69.6) bone fill. The elevated heterogeneity $\left(I^{2}=77.3 \%\right)$ between the studies was partially explained by the estimated level of methodological risk of bias (low/moderate risk of bias: SMD $=-2.03,95 \% \mathrm{Cl}$ : $-2.47,-1.59, P$-value $<0.001,{ }^{2}=$ $59.4 \%, 14$ studies; high risk of bias: $\mathrm{SMD}=-1.77,95 \% \mathrm{Cl}:-2.24,-1.29, P$-value $<0.001, I^{2}$ $=83.4 \%, 11$ studies). 
Chapter 2

Table S2 Characteristics of included studies.

\begin{tabular}{|c|c|c|c|c|c|c|c|c|c|}
\hline ID & Author & Year & Study Design & Exposure / Intervention & $\begin{array}{l}\mathrm{N} \\
\text { Patients }\end{array}$ & $\mathrm{N}$ Clefts & $\begin{array}{l}\text { Cleft } \\
\text { Type }\end{array}$ & $\begin{array}{l}\text { Average } \\
\text { Age }\end{array}$ & $\begin{array}{l}\text { Imaging } \\
\text { Modality }\end{array}$ \\
\hline \multirow[t]{2}{*}{1} & Alonso et al. & 2010 & $\mathrm{RCT}$ & $\begin{array}{l}\text { resorbable collagen matrix } \\
\text { with rhBMP-2 (Infuse Bone } \\
\text { Graft) }\end{array}$ & 8 & 8 & UCL & $\begin{array}{l}9 \text { year } 6 \\
\text { month }\end{array}$ & $\begin{array}{l}\text { conventional } \\
\text { computed } \\
\text { tomography }\end{array}$ \\
\hline & & & & $\begin{array}{l}\text { conventional iliac crest bone } \\
\text { graft }\end{array}$ & 8 & 8 & UCL & $\begin{array}{l}9 \text { year } 6 \\
\text { month }\end{array}$ & $\begin{array}{l}\text { conventional } \\
\text { computed } \\
\text { tomography }\end{array}$ \\
\hline \multirow[t]{3}{*}{2} & $\begin{array}{l}\text { Canan Jr } \\
\text { et al. }\end{array}$ & 2012 & $\mathrm{RCT}$ & Perioplasty & 6 & 6 & UCL & $\begin{array}{l}9 \text { year } 5 \\
\text { month }\end{array}$ & $\begin{array}{l}\text { conventiona } \\
\text { computed } \\
\text { tomography }\end{array}$ \\
\hline & & & & ICBG & 6 & 6 & UCL & $\begin{array}{l}10 \text { year } 8 \\
\text { month }\end{array}$ & $\begin{array}{l}\text { conventional } \\
\text { computed } \\
\text { tomography }\end{array}$ \\
\hline & & & & $\begin{array}{l}\text { resorbable collagen sponge } \\
\text { with rhBMP-2 (Infuse Bone } \\
\text { Graft) }\end{array}$ & 6 & 6 & UCL & $\begin{array}{l}8 \text { year } 7 \\
\text { month }\end{array}$ & $\begin{array}{l}\text { conventiona } \\
\text { computed } \\
\text { tomography }\end{array}$ \\
\hline
\end{tabular}

\begin{tabular}{|c|c|c|c|c|c|c|c|c|c|}
\hline 3 & $\begin{array}{l}\text { de Ruiter } \\
\text { et al. }\end{array}$ & 2015 & prospective & $\begin{array}{l}\text { micro-structured beta- } \\
\text { tricalcium phosphate mixes } \\
\text { with autologous blood ( } 65 \% \\
+/-15 \% \text { porous and }>90 \% \text { pure } \\
\text { phase B-TCP) }\end{array}$ & 7 & $\begin{array}{l}6 \\
\text { included }\end{array}$ & $U C L$ & $\begin{array}{l}11 \text { years } \\
1.1 \mathrm{~m}\end{array}$ & $\begin{array}{l}\text { Cone Beam } \\
\text { Computed } \\
\text { Tomography }\end{array}$ \\
\hline \multirow[t]{2}{*}{4} & $\begin{array}{l}\text { Dickinson } \\
\text { et al. }\end{array}$ & 2008 & $\mathrm{RCT}$ & $\begin{array}{l}\text { resorbable collagen matrix } \\
\text { with rhBMP-2 (Infuse Bone } \\
\text { Graft) }\end{array}$ & 9 & 9 & UCL & $16.4(1.5)$ & $\begin{array}{l}\text { Cone Beam } \\
\text { Computed } \\
\text { Tomography }\end{array}$ \\
\hline & & & & $\begin{array}{l}\text { conventional iliac crest bone } \\
\text { graft }\end{array}$ & 12 & 12 & UCL & $15.9(1.9)$ & $\begin{array}{l}\text { Cone Beam } \\
\text { Computed } \\
\text { Tomography }\end{array}$ \\
\hline 5 & $\begin{array}{l}\text { Dieleman } \\
\text { et al. }\end{array}$ & 2004 & prospective & $\begin{array}{l}\text { conventional iliac crest bone } \\
\text { graft }\end{array}$ & 5 & 5 & $U C L$ & $9 y 6 m$ & $\begin{array}{l}\text { conventional } \\
\text { computed } \\
\text { tomography }\end{array}$ \\
\hline
\end{tabular}

\begin{tabular}{|c|c|c|c|c|c|c|c|c|c|}
\hline \multirow[t]{2}{*}{6} & Dissaux et al. & 2016 & retrospective & $\begin{array}{l}\text { conventional iliac crest bone } \\
\text { graft }\end{array}$ & 14 & 15 & $\begin{array}{l}\mathrm{UCL} \\
+ \\
\mathrm{BCL}\end{array}$ & $\begin{array}{l}5 \text { years } 2 \\
\text { month }\end{array}$ & $\begin{array}{l}\text { Cone Beam } \\
\text { Computed } \\
\text { Tomography }\end{array}$ \\
\hline & & & & $\begin{array}{l}\text { conventional iliac crest bone } \\
\text { graft }\end{array}$ & 14 & 16 & $\begin{array}{l}\text { UCL } \\
+ \\
B C L\end{array}$ & 10 years & $\begin{array}{l}\text { Cone Beam } \\
\text { Computed } \\
\text { Tomography }\end{array}$ \\
\hline 7 & $\begin{array}{l}\text { Feichtinger } \\
\text { et al. }\end{array}$ & 2007 & prospective & $\begin{array}{l}\text { conventional iliac crest bone } \\
\text { graft }\end{array}$ & 24 & 24 & $\cup C L$ & $\begin{array}{l}10 \text { year } 6 \\
\text { month }\end{array}$ & $\begin{array}{l}\text { conventional } \\
\text { computed } \\
\text { tomography }\end{array}$ \\
\hline 8 & $\begin{array}{l}\text { Feichtinger } \\
\text { et al. }\end{array}$ & 2008 & prospective & $\begin{array}{l}\text { conventional iliac crest bone } \\
\text { graft }\end{array}$ & 20 & 20 & $U C L$ & 11 year & $\begin{array}{l}\text { conventional } \\
\text { computed } \\
\text { tomography }\end{array}$ \\
\hline
\end{tabular}




\begin{tabular}{|c|c|c|c|c|c|c|c|c|c|}
\hline \multirow{2}{*}{$\begin{array}{l}\text { Method of } \\
\text { Volumetric } \\
\text { Analysis }\end{array}$} & \multirow{2}{*}{$\begin{array}{l}\text { Average } \\
\text { Follow-up }\end{array}$} & \multicolumn{2}{|c|}{ Preoperative Cleft } & \multicolumn{2}{|c|}{ Postoperative Cleft } & \multicolumn{3}{|c|}{ Percentage Bone Fill } & \multirow[t]{2}{*}{$\mathrm{p}$-value } \\
\hline & & $\begin{array}{l}\text { Raw } \\
\text { Volume }\end{array}$ & Raw SD & $\begin{array}{l}\text { Raw } \\
\text { Volume }\end{array}$ & Raw SD & $\begin{array}{l}\text { Raw } \\
\text { Reported \% }\end{array}$ & $\begin{array}{l}\% \text { Bone } \\
\text { Fill }\end{array}$ & SD & \\
\hline $\begin{array}{l}\text { Osirix DICOM } \\
\text { Viewer }\end{array}$ & $6 m+1$ year & $974.8 \mathrm{~mm} 3$ & 236.8 & $\begin{array}{l}\text { mean } \\
\text { defect } \\
247.1 \\
\text { mm3 }\end{array}$ & 112.8 & $\begin{array}{l}\text { bone fill } \\
74.4 \%\end{array}$ & $74.4 \%$ & 10.8 & 0,176 \\
\hline $\begin{array}{l}\text { Osirix DICOM } \\
\text { Viewer }\end{array}$ & $6 m+1$ year & $1052.4 \mathrm{~mm} 3$ & 326.0 & $\begin{array}{l}\text { mean } \\
\text { defect } \\
207.8 \\
\text { mm3 }\end{array}$ & 77.9 & $\begin{array}{l}\text { bone fill } \\
80.2 \%\end{array}$ & $80.2 \%$ & 4.1 & - \\
\hline $\begin{array}{l}\text { Kodak Carestream } \\
\text { PACS }\end{array}$ & $\begin{array}{l}3 \mathrm{~m}, 6 \mathrm{~m}, \\
12 \mathrm{~m}\end{array}$ & $430.4 \mathrm{~mm} 3$ & & - & & & & & \\
\hline $\begin{array}{l}\text { Kodak Carestream } \\
\text { PACS }\end{array}$ & $\begin{array}{l}3 \mathrm{~m}, 6 \mathrm{~m} \\
12 \mathrm{~m}\end{array}$ & $656.9 \mathrm{~mm} 3$ & & $\begin{array}{l}\text { bone } \\
\text { forming } \\
\text { volume } \\
520.5 \\
\text { mm3 }\end{array}$ & 310.1 & $\begin{array}{l}\text { bone } \\
\text { formation } \\
78 \%\end{array}$ & $78 \%$ & 15.1 & 0,937 \\
\hline $\begin{array}{l}\text { Kodak Carestream } \\
\text { PACS }\end{array}$ & $\begin{array}{l}3 \mathrm{~m}, 6 \mathrm{~m} \\
12 \mathrm{~m}\end{array}$ & $471.8 \mathrm{~mm} 3$ & & $\begin{array}{l}\text { bone } \\
\text { forming } \\
\text { volume } \\
354.4 \\
\text { mm3 }\end{array}$ & 130.7 & $\begin{array}{l}\text { bone } \\
\text { formation } \\
75.1 \%\end{array}$ & $75.1 \%$ & 20.6 & \\
\hline $\begin{array}{l}\text { Osirix DICOM } \\
\text { Viewer }\end{array}$ & $1 \mathrm{w}, 6 \mathrm{~m}$ & $0.72 \mathrm{~cm} 3$ & 0.26 & $\begin{array}{l}\text { bone } \\
\text { volume } \\
0.53 \mathrm{~cm} 3\end{array}$ & 0.21 & $\begin{array}{l}\text { Bone volume } \\
73 \%\end{array}$ & $73 \%$ & 6 & \\
\hline $\begin{array}{l}\text { Imagej program } \\
\text { (NIH) }\end{array}$ & 1 year & $5.6 \mathrm{cc}$ & 0.3 & $\begin{array}{l}\text { defect } \\
0.3 c c\end{array}$ & 0.02 & bone fill 95\% & $95 \%$ & & 0.01 \\
\hline $\begin{array}{l}\text { Imagej program } \\
(\mathrm{NIH})\end{array}$ & 1 year & $5.1 \mathrm{cc}$ & 0.4 & 1.9 & 0.2 & bone fill 63\% & $63 \%$ & & \\
\hline $\begin{array}{l}\text { Siemens Somatom } \\
\text { workstation }\end{array}$ & 1 year & $\begin{array}{l}2.15 \mathrm{~cm} 3 \\
\text { (bone vol } \\
\text { immediate } \\
\text { postop) }\end{array}$ & & $\begin{array}{l}1.62 \mathrm{~cm} 3 \\
\text { (bone vol) }\end{array}$ & & $75 \%$ & $75 \%$ & & \\
\hline $\begin{array}{l}\text { Osirix DICOM } \\
\text { Viewer }\end{array}$ & $2105286 \mathrm{~m}$ & $0.965 \mathrm{~cm} 3$ & $(0.7-1.3)$ & $0.6 \mathrm{~cm} 3$ & $\begin{array}{l}(0.36- \\
1.1)\end{array}$ & $\begin{array}{l}\text { Bone volume } \\
63.3 \%\end{array}$ & $63.3 \%$ & & 0.012 \\
\hline $\begin{array}{l}\text { Osirix DICOM } \\
\text { Viewer }\end{array}$ & $2105286 \mathrm{~m}$ & $1.03 \mathrm{~cm} 3$ & $(0.7-1.5)$ & $0.45 \mathrm{~cm} 3$ & $(0-0.7)$ & $46.2 \%$ & $46.2 \%$ & - & - \\
\hline $\begin{array}{l}\text { STN-Zeiss- } \\
\text { Navigation System }\end{array}$ & $1 y, 2 y, 3 y$ & $1.17 \mathrm{~cm} 3$ & 0.31 & $0.56 \mathrm{~cm} 3$ & $\begin{array}{l}0.41 \\
\mathrm{~cm} 3\end{array}$ & $\begin{array}{l}\text { averge } \\
\text { volume graft } \\
50.21\end{array}$ & $50.21 \%$ & 34.59 & - \\
\hline $\begin{array}{l}\text { STN-Zeiss- } \\
\text { Navigation System }\end{array}$ & $1 y, 2 y$ & $1.2 \mathrm{~cm} 3$ & 0.3 & $0.6 \mathrm{~cm} 3$ & 0.4 & $\begin{array}{l}\text { graft volume } \\
50 \%\end{array}$ & $50 \%$ & 37 & \\
\hline
\end{tabular}


Chapter 2

Table S2 Continued.

\begin{tabular}{|c|c|c|c|c|c|c|c|c|c|}
\hline ID & Author & Year & Study Design & Exposure / Intervention & $\begin{array}{l}\mathrm{N} \\
\text { Patients }\end{array}$ & $\mathrm{N}$ Clefts & $\begin{array}{l}\text { Cleft } \\
\text { Type }\end{array}$ & $\begin{array}{l}\text { Average } \\
\text { Age }\end{array}$ & $\begin{array}{l}\text { Imaging } \\
\text { Modality }\end{array}$ \\
\hline \multirow[t]{2}{*}{9} & Herford et al. & 2007 & retrospective & $\begin{array}{l}\text { resorbable type I bovine } \\
\text { collagen matrix with rhBMP-2 } \\
\text { (Infuse Bone Graft) }\end{array}$ & 10 & 10 & $U C L$ & $8 y$ & $\begin{array}{l}\text { conventional } \\
\text { computed } \\
\text { tomography }\end{array}$ \\
\hline & & & & $\begin{array}{l}\text { conventional iliac crest bone } \\
\text { graft }\end{array}$ & 2 & 2 & $\cup C L$ & $10 y$ & $\begin{array}{l}\text { conventional } \\
\text { computed } \\
\text { tomography }\end{array}$ \\
\hline 10 & Honma et al. & 1999 & retrospective & $\begin{array}{l}\text { conventional iliac crest bone } \\
\text { graft }\end{array}$ & 15 & 15 & $U C L$ & 10 y $1 \mathrm{~m}$ & $\begin{array}{l}\text { conventional } \\
\text { computed } \\
\text { tomography }\end{array}$ \\
\hline 11 & Hudak et al. & 2014 & retrospective & $\begin{array}{l}\text { cranial bone graft - shavings + } \\
\text { osteotomes }\end{array}$ & 10 & NS & $\begin{array}{l}\text { UCL } \\
+ \\
\mathrm{BCL}\end{array}$ & 10 year & $\begin{array}{l}\text { limited } \\
\text { computed } \\
\text { tomography }\end{array}$ \\
\hline
\end{tabular}

\begin{tabular}{|c|c|c|c|c|c|c|c|c|c|}
\hline 12 & Hussain & 2013 & retrospective & Tibial graft & 9 & - & & $17.78 \mathrm{yrs}$ & $\begin{array}{l}\text { conventional } \\
\text { computed } \\
\text { tomography }\end{array}$ \\
\hline 13 & $\begin{array}{l}\text { Linderup } \\
\text { et al. }\end{array}$ & 2016 & retrospective & $\begin{array}{l}\text { Mandibular Smphyseal Bone } \\
\text { graft }\end{array}$ & 32 & 32 & UCL & $9 y 6 m$ & $\begin{array}{l}\text { Cone Beam } \\
\text { Computed } \\
\text { Tomography }\end{array}$ \\
\hline
\end{tabular}

\begin{tabular}{|c|c|c|c|c|c|c|c|c|c|}
\hline 14 & $\begin{array}{l}\text { Nagashima } \\
\text { et al. }\end{array}$ & 2014 & retrospective & $\begin{array}{l}\text { conventional iliac crest bone } \\
\text { graft }\end{array}$ & 29 & 29 & $\mathrm{UCL}$ & $10 y 1 \mathrm{~m}$ & $\begin{array}{l}\text { conventional } \\
\text { computed } \\
\text { tomography }\end{array}$ \\
\hline 15 & $\begin{array}{l}\text { Neovius } \\
\text { et al. }\end{array}$ & 2013 & $\mathrm{RCT}$ & $\begin{array}{l}\text { BMP-2 in hydrogel (low } \\
\text { concentration 50microg) }\end{array}$ & 2 & 2 & $\mathrm{UCL}$ & $9 y 6 m$ & $\begin{array}{l}\text { conventional } \\
\text { computed } \\
\text { tomography }\end{array}$ \\
\hline
\end{tabular}

\begin{tabular}{|c|c|c|c|c|c|c|}
\hline RCT & $\begin{array}{l}\text { BMP-2 in hydrogel (high } \\
\text { concentration 250microg) }\end{array}$ & 2 & 2 & UCL & $9 y 6 \mathrm{~m}$ & $\begin{array}{l}\text { conventional } \\
\text { computed } \\
\text { tomography }\end{array}$ \\
\hline RCT & $\begin{array}{l}\text { conventional iliac crest bone } \\
\text { graft }\end{array}$ & 3 & 3 & UCL & $9 y 10 m$ & $\begin{array}{l}\text { conventional } \\
\text { computed } \\
\text { tomography }\end{array}$ \\
\hline
\end{tabular}

\begin{tabular}{|c|c|c|c|c|c|c|c|c|c|}
\hline 16 & Oberoi et al. & 2009 & Prospective & $\begin{array}{l}\text { conventional iliac crest bone } \\
\text { graft }\end{array}$ & 21 & $\begin{array}{l}(4 \times 2+ \\
17)=25\end{array}$ & $\begin{array}{l}\mathrm{UCL} \\
+ \\
\mathrm{BCL}\end{array}$ & $10 y 7 m$ & $\begin{array}{l}\text { Cone Beam } \\
\text { Computed } \\
\text { Tomography }\end{array}$ \\
\hline
\end{tabular}




\begin{tabular}{|c|c|c|c|c|c|c|c|c|c|}
\hline \multirow{2}{*}{$\begin{array}{l}\text { Method of } \\
\text { Volumetric } \\
\text { Analysis }\end{array}$} & \multirow{2}{*}{$\begin{array}{l}\text { Average } \\
\text { Follow-up }\end{array}$} & \multicolumn{2}{|c|}{ Preoperative Cleft } & \multicolumn{2}{|c|}{ Postoperative Cleft } & \multicolumn{3}{|c|}{ Percentage Bone Fill } & \multirow[t]{2}{*}{$\mathrm{p}$-value } \\
\hline & & $\begin{array}{l}\text { Raw } \\
\text { Volume }\end{array}$ & Raw SD & $\begin{array}{l}\text { Raw } \\
\text { Volume }\end{array}$ & Raw SD & $\begin{array}{l}\text { Raw } \\
\text { Reported \% }\end{array}$ & $\begin{array}{l}\% \text { Bone } \\
\text { Fill }\end{array}$ & SD & \\
\hline $\begin{array}{l}\text { IMPAX; Afga- } \\
\text { Gavaert }\end{array}$ & $2105284 m$ & $10.55 \mathrm{~cm} 3$ & & $\begin{array}{l}7.57 \mathrm{~cm} 3 \\
\text { bone } \\
\text { volume }\end{array}$ & & $\begin{array}{l}\text { Bone fill } \\
71.7 \%\end{array}$ & $71.7 \%$ & & \\
\hline $\begin{array}{l}\text { IMPAX; Afga- } \\
\text { Gavaert }\end{array}$ & $2105284 m$ & $17.86 \mathrm{~cm} 3$ & & $13.37 \mathrm{~cm} 3$ & & $78.1 \%$ & $78.1 \%$ & & \\
\hline manual calculation & $3 \mathrm{~m}, 1$ year & $1.1 \mathrm{~cm} 3$ & $0.3 \mathrm{~cm} 3$ & $\begin{array}{l}1.1 \mathrm{~cm} 3 \\
\text { bone } \\
\text { volume }\end{array}$ & $0.5 \mathrm{~cm} 3$ & $\begin{array}{l}\text { Graft volume } \\
99 \%\end{array}$ & $99 \%$ & 38 & - \\
\hline $\begin{array}{l}\text { Amira volume- } \\
\text { rendering software }\end{array}$ & 1 year & $1.19 \mathrm{ml}$ & $\begin{array}{l}0.52- \\
1.82 \mathrm{ml} \\
\text { range }\end{array}$ & $\begin{array}{l}0.19 \mathrm{ml} \\
\text { average } \\
\text { defect } \\
\text { volume } \\
\text { post op }\end{array}$ & $\begin{array}{l}0.06-.57 \\
\mathrm{ml}\end{array}$ & bone fill 85\% & $85 \%$ & & \\
\hline $\begin{array}{l}\text { Siemens Somatam } \\
\text { Espirit inbuilt } \\
\text { software }\end{array}$ & $6 m$ & - & - & - & - & $\begin{array}{l}\text { bone fill } \\
73.15 \%\end{array}$ & $73.15 \%$ & 9.9 & - \\
\hline $\begin{array}{l}\text { Mimics 15.0, } \\
\text { Materialise } \\
\text { Interactive Medical } \\
\text { Image Control } \\
\text { System }\end{array}$ & 1 year & $934 \mathrm{~mm} 3$ & $296 \mathrm{~mm} 3$ & $\begin{array}{l}111 \mathrm{~mm} 3 \\
\text { defect }\end{array}$ & $\begin{array}{l}122 \\
\mathrm{~mm} 3\end{array}$ & bone fill $87 \%$ & $87 \%$ & $14 \%$ & 0.05 \\
\hline $\begin{array}{l}\text { DICOM manager } \\
\text { (ZedView, LEXI Inc., } \\
\text { Tokyo, Japan) }\end{array}$ & $2105286 m$ & $1.16 \mathrm{~cm} 3$ & $0.42 \mathrm{~cm} 3$ & $\begin{array}{l}\text { remaining } \\
\text { bone } 0.56 \\
\mathrm{~cm} 3\end{array}$ & 0.40 & $\begin{array}{l}48.28 \% \\
\text { calculated }\end{array}$ & $48.28 \%$ & - & - \\
\hline $\begin{array}{l}\text { General Electric } \\
\text { Advantage } \\
\text { Windows } \\
\text { workstation } \\
\text { Volume viewer } \\
\text { 4.5_02 }\end{array}$ & $2105286 m$ & $2.20 \mathrm{~cm} 3$ & & $1.91 \mathrm{~cm} 3$ & & $\begin{array}{l}\text { bone fill } \\
12 \%\end{array}$ & $12 \%$ & & \\
\hline $\begin{array}{l}\text { General Electric } \\
\text { Advantage } \\
\text { Windows } \\
\text { workstation } \\
\text { Volume viewer } \\
\text { 4.5_02 }\end{array}$ & $2105286 m$ & $1.59 \mathrm{~cm} 3$ & & $0.79 \mathrm{~cm} 3$ & & bone fill $46 \%$ & $46 \%$ & & \\
\hline $\begin{array}{l}\text { General Electric } \\
\text { Advantage } \\
\text { Windows } \\
\text { workstation } \\
\text { Volume viewer } \\
4.5 \_02\end{array}$ & $2105286 m$ & $1.71 \mathrm{~cm} 3$ & & $0.93 \mathrm{~cm} 3$ & & $\begin{array}{l}\text { bone fill } \\
48.67 \%\end{array}$ & $48.67 \%$ & & \\
\hline $\begin{array}{l}\text { Amira volume- } \\
\text { rendering software } \\
\text { 3.1.1 }\end{array}$ & 1 year & $0.7 \mathrm{~cm} 3$ & $\begin{array}{l}(\mathrm{UCL} \\
0.61 \mathrm{~cm} 3 \\
+\mathrm{BCL} \\
0.82 \mathrm{~cm} 3)\end{array}$ & $\begin{array}{l}(U C L L \\
0.08 \mathrm{~cm} 3 \\
+B C L \\
0.21 \mathrm{~cm} 3)\end{array}$ & & bone fill $84 \%$ & $84 \%$ & & \\
\hline
\end{tabular}


Chapter 2

Table S2 Continued.

\begin{tabular}{|c|c|c|c|c|c|c|c|c|c|}
\hline ID & Author & Year & Study Design & Exposure / Intervention & $\begin{array}{l}\mathrm{N} \\
\text { Patients }\end{array}$ & $\mathrm{N}$ Clefts & $\begin{array}{l}\text { Cleft } \\
\text { Type }\end{array}$ & $\begin{array}{l}\text { Average } \\
\text { Age }\end{array}$ & $\begin{array}{l}\text { Imaging } \\
\text { Modality }\end{array}$ \\
\hline \multirow[t]{2}{*}{17} & Oyama et al. & 2004 & retrospective & ICBG + PRP + Fibrin glue & 7 & 7 & $\cup C L$ & $\begin{array}{l}16 y 1 \\
\text { month }\end{array}$ & $\begin{array}{l}\text { conventional } \\
\text { computed } \\
\text { tomography }\end{array}$ \\
\hline & & & & $\begin{array}{l}\text { conventional iliac crest bone } \\
\text { graft }\end{array}$ & 5 & 5 & $\cup C L$ & $\begin{array}{l}16 y 4 \\
\text { months }\end{array}$ & $\begin{array}{l}\text { conventional } \\
\text { computed } \\
\text { tomography }\end{array}$ \\
\hline 18 & Ozawa et al. & 2007 & retrospective & $\begin{array}{l}\text { conventional iliac crest bone } \\
\text { graft }\end{array}$ & 25 & 35 & $\begin{array}{l}\mathrm{UCL} \\
+ \\
\mathrm{BCL}\end{array}$ & $6 y 8 m$ & $\begin{array}{l}\text { conventional } \\
\text { computed } \\
\text { tomography }\end{array}$ \\
\hline \multirow[t]{2}{*}{19} & Pradel et al. & 2012 & prospective & $\begin{array}{l}\text { autogenous Osteoblasts } \\
\text { cultured on demineralised } \\
\text { bone matrix Osteovit }\end{array}$ & 4 & 4 & $U C L$ & $11 \mathrm{y} 2 \mathrm{~m}$ & $\begin{array}{l}\text { Cone Beam } \\
\text { Computed } \\
\text { Tomography }\end{array}$ \\
\hline & & & prospective & $\begin{array}{l}\text { conventional iliac crest bone } \\
\text { graft }\end{array}$ & 4 & 5 & $\begin{array}{l}\mathrm{UCL} \\
+ \\
\mathrm{BCL}\end{array}$ & $9 y 5 m$ & $\begin{array}{l}\text { Cone Beam } \\
\text { Computed } \\
\text { Tomography }\end{array}$ \\
\hline 20 & Reddy et al. & 2015 & prospective & $\begin{array}{l}\text { conventional iliac crest bone } \\
\text { graft }\end{array}$ & 10 & 10 & & $9 y 7 m$ & $\begin{array}{l}\text { conventional } \\
\text { computed } \\
\text { tomography }\end{array}$ \\
\hline 21 & Rychlik et al. & 2012 & retrospective & $\begin{array}{l}\text { conventional iliac crest bone } \\
\text { graft }\end{array}$ & 62 & 62 & & $11 y$ & $\begin{array}{l}\text { conventional } \\
\text { computed } \\
\text { tomography }\end{array}$ \\
\hline \multirow[t]{2}{*}{22} & Shawky et al. & 2015 & $\mathrm{RCT}$ & $\begin{array}{l}\text { PRF (Platelet rich fibrin) }+ \\
\text { conventional iliac crest bone } \\
\text { graft }\end{array}$ & 12 & 12 & & $10 y 9 m$ & $\begin{array}{l}\text { conventional } \\
\text { computed } \\
\text { tomography }\end{array}$ \\
\hline & & & RCT & $\begin{array}{l}\text { conventional iliac crest bone } \\
\text { graft }\end{array}$ & 12 & 12 & & $10 y 9 m$ & $\begin{array}{l}\text { conventional } \\
\text { computed } \\
\text { tomography }\end{array}$ \\
\hline 23 & Tai et al. & 2000 & prospective & $\begin{array}{l}\text { conventional iliac crest bone } \\
\text { graft }\end{array}$ & $\begin{array}{l}14(11 \\
U C L+3 \\
B L P)\end{array}$ & 17 & & $9 y 1 \mathrm{~m}$ & $\begin{array}{l}\text { conventional } \\
\text { computed } \\
\text { tomography }\end{array}$ \\
\hline \multirow[t]{2}{*}{24} & $\begin{array}{l}\text { Takemaru } \\
\text { et al. }\end{array}$ & 2016 & $\mathrm{RCT}$ & $\begin{array}{l}\text { conventional iliac crest bone } \\
\text { graft }\end{array}$ & 10 & 10 & $\cup C L$ & $8 y 4 m$ & $\begin{array}{l}\text { conventional } \\
\text { computed } \\
\text { tomography }\end{array}$ \\
\hline & & & $\mathrm{RCT}$ & $\begin{array}{l}\text { bioabsorbable hydroxyapatite } \\
\text { and collagen complex (0.5 ml } \\
\text { HA/Col, RIFIT, Hoya Co, Tokyo, } \\
\text { Japan) }\end{array}$ & 5 & 5 & $\cup C L$ & $8 y 6 m$ & $\begin{array}{l}\text { conventional } \\
\text { computed } \\
\text { tomography }\end{array}$ \\
\hline 25 & $\begin{array}{l}\text { Touzet- } \\
\text { Roumazeille } \\
\text { et al. }\end{array}$ & 2015 & retrospective & $\begin{array}{l}\text { conventional iliac crest bone } \\
\text { graft + gengivoperiosteoplasty }\end{array}$ & 37 & 37 & & $5 y 5 m$ & $\begin{array}{l}\text { Cone Beam } \\
\text { Computed } \\
\text { Tomography }\end{array}$ \\
\hline 26 & Zahng et al. & 2012 & prospective & $\begin{array}{l}\text { conventional iliac crest bone } \\
\text { graft }\end{array}$ & 19 & 19 & & $15 y 8 m$ & $\begin{array}{l}\text { Cone Beam } \\
\text { Computed } \\
\text { Tomography }\end{array}$ \\
\hline
\end{tabular}




\begin{tabular}{|c|c|c|c|c|c|c|c|c|c|}
\hline \multirow{2}{*}{$\begin{array}{l}\text { Method of } \\
\text { Volumetric } \\
\text { Analysis }\end{array}$} & \multirow{2}{*}{$\begin{array}{l}\text { Average } \\
\text { Follow-up }\end{array}$} & \multicolumn{2}{|c|}{ Preoperative Cleft } & \multicolumn{2}{|c|}{ Postoperative Cleft } & \multicolumn{3}{|c|}{ Percentage Bone Fill } & \multirow[t]{2}{*}{$\mathrm{p}$-value } \\
\hline & & $\begin{array}{l}\text { Raw } \\
\text { Volume }\end{array}$ & Raw SD & $\begin{array}{l}\text { Raw } \\
\text { Volume }\end{array}$ & Raw SD & $\begin{array}{l}\text { Raw } \\
\text { Reported \% }\end{array}$ & $\begin{array}{l}\% \text { Bone } \\
\text { Fill }\end{array}$ & SD & \\
\hline Adobe Photoshop & $2105286 m$ & $\begin{array}{l}\text { Reported in } \\
\text { pixels }\end{array}$ & - & & & $\begin{array}{l}\text { bone fill } \\
80.19 \%\end{array}$ & $80.19 \%$ & 6.77 & 0.05 \\
\hline Adobe Photoshop & $2105286 m$ & $\begin{array}{l}\text { Reported in } \\
\text { pixels }\end{array}$ & - & - & - & $\begin{array}{l}\text { average } \\
63,67 \%\end{array}$ & $63.67 \%$ & 13.94 & - \\
\hline $\begin{array}{l}\text { SIMPlant } \\
\text { (Materialize, NV) }\end{array}$ & $2105286 m$ & $\begin{array}{l}1.37 \mathrm{ml} \\
\text { (immediate } \\
\text { postop) }\end{array}$ & $0.51 \mathrm{ml}$ & $\begin{array}{l}0.73 \mathrm{ml} \\
\text { (remaining } \\
\text { graft } \\
\text { volume) }\end{array}$ & $.26 \mathrm{ml}$ & $\begin{array}{l}53.28 \% \\
\text { calculated }\end{array}$ & $53.28 \%$ & - & \\
\hline $\begin{array}{l}\text { iPlan 2.6 Cranial, } \\
\text { BrainLab, } \\
\text { Germany) }\end{array}$ & $2105286 \mathrm{~m}$ & $0.93 \mathrm{~cm} 3$ & $0.23 \mathrm{~cm} 3$ & $\begin{array}{l}\text { cleft } \\
\text { volume } \\
0.55 \mathrm{~cm} 3\end{array}$ & $\begin{array}{l}0.24 \\
\mathrm{~cm} 3\end{array}$ & $\begin{array}{l}\text { bone fill } \\
40.9 \%\end{array}$ & $40.9 \%$ & - & - \\
\hline $\begin{array}{l}\text { iPlan 2.6 Cranial, } \\
\text { BrainLab, } \\
\text { Germany) }\end{array}$ & $2105286 m$ & $0.93 \mathrm{~cm} 3$ & $0.36 \mathrm{~cm} 3$ & $\begin{array}{l}\text { cleft } \\
\text { volume } \\
0.59 \mathrm{~cm} 3\end{array}$ & $\begin{array}{l}0.23 \\
\mathrm{~cm} 3\end{array}$ & $\begin{array}{l}\text { bone fill } \\
36.6 \%\end{array}$ & $36.6 \%$ & - & \\
\hline & $2105286 m$ & $0.805 \mathrm{~cm} 3$ & $0.36 \mathrm{~cm} 3$ & $\begin{array}{l}0.546 \mathrm{~cm} 3 \\
\text { (bone } \\
\text { volume) }\end{array}$ & 0.34 & $\begin{array}{l}67.83 \% \\
\text { calculated }\end{array}$ & $67.83 \%$ & - & \\
\hline $\begin{array}{l}\text { Siemens } \\
\text { SOMATOM } \\
\text { Emotion software }\end{array}$ & $2105286 \mathrm{~m}$ & $\begin{array}{l}2.15 \mathrm{~cm} 3 \\
\text { (bone } \\
\text { volume } \\
\text { immediate } \\
\text { postop) }\end{array}$ & 0.75 & $\begin{array}{l}0.56 \mathrm{~cm} 3 \\
\text { (bone } \\
\text { volume) }\end{array}$ & 0.29 & $\begin{array}{l}26 \% \\
\text { calculated }\end{array}$ & $26 \%$ & & \\
\hline $\begin{array}{l}\text { DicomWorks } 1.3 .5 \\
\text { (\{hilippe Puech and } \\
\text { Loic Boussel) }\end{array}$ & $2105286 m$ & $0.96 \mathrm{~cm} 3$ & $\begin{array}{l}0.259 \\
\mathrm{~cm} 3\end{array}$ & $\begin{array}{l}0.18 \mathrm{~cm} 3 \\
\text { (defect } \\
\text { vol) }\end{array}$ & $\begin{array}{l}0.08 \\
\mathrm{~cm} 3\end{array}$ & $\begin{array}{l}\text { new bone } \\
82.61 \%\end{array}$ & $82.61 \%$ & $3,91 \%$ & 0.5 \\
\hline $\begin{array}{l}\text { DicomWorks } 1.3 .5 \\
\text { (\{hilippe Puech and } \\
\text { Loic Boussel) }\end{array}$ & $2105286 m$ & $0.9 \mathrm{~cm} 3$ & $0.28 \mathrm{~cm} 3$ & $0.275 \mathrm{~cm} 3$ & $\begin{array}{l}0.05 \\
\mathrm{~cm} 3\end{array}$ & $\begin{array}{l}\text { new bone } \\
68,38 \%\end{array}$ & $68.38 \%$ & $6,67 \%$ & \\
\hline $\begin{array}{l}\text { Siemens Somatom } \\
\text { workstation }\end{array}$ & 1 year & $\begin{array}{l}2.1 \mathrm{~cm} 3 \\
\text { (immediate } \\
\text { post volume) }\end{array}$ & $0.7 \mathrm{~cm} 3$ & $\begin{array}{l}1.20 \mathrm{~cm} 3 \\
\text { (residual } \\
\text { bone } \\
\text { volume) }\end{array}$ & $0.44 \mathrm{~cm} 3$ & $\begin{array}{l}56.9 \%(43.1 \% \\
\text { volume loss) }\end{array}$ & $56.9 \%$ & & \\
\hline $\begin{array}{l}\text { DICOM manager } \\
\text { (ZedView, LEXI Inc., } \\
\text { Tokyo, Japan) }\end{array}$ & 1 year & $0.869 \mathrm{ml}$ & $0.221 \mathrm{ml}$ & $\begin{array}{l}0.518 \mathrm{ml} \\
\text { (bone } \\
\text { volume) }\end{array}$ & $0.303 \mathrm{ml}$ & $\begin{array}{l}59.6 \% \\
\text { calculated }\end{array}$ & $59.6 \%$ & - & \\
\hline $\begin{array}{l}\text { DICOM manager } \\
\text { (ZedView, LEXI Inc., } \\
\text { Tokyo, Japan) }\end{array}$ & 1 year & $0.884 \mathrm{ml}$ & $0.116 \mathrm{ml}$ & $\begin{array}{l}0.648 \mathrm{ml} \\
\text { (bone } \\
\text { volume) }\end{array}$ & $0.120 \mathrm{ml}$ & $\begin{array}{l}73.3 \% \\
\text { calculated }\end{array}$ & $73.3 \%$ & - & - \\
\hline $\begin{array}{l}\text { Osirix DICOM } \\
\text { Viewer }\end{array}$ & 7.4 months & $1.29 \mathrm{~cm} 3$ & $0.43 \mathrm{~cm} 3$ & $\begin{array}{l}\text { graft } \\
\text { volume } \\
0.73 \mathrm{~cm} 3\end{array}$ & $\begin{array}{l}0.23 \\
\mathrm{~cm} 3\end{array}$ & $\begin{array}{l}\text { bone fill } \\
61.89 \%\end{array}$ & $61.89 \%$ & 18.77 & - \\
\hline $\begin{array}{l}\text { SIMPlant Pro } \\
11.4 \text { (Materialize, } \\
\text { Belgium) }\end{array}$ & $2105286 m$ & $2.52 \mathrm{~cm} 3$ & 0.58 & $\begin{array}{l}1.78 \mathrm{~cm} 3 \\
\text { (bone } \\
\text { volume) }\end{array}$ & $\begin{array}{l}0.23 \\
\mathrm{~cm} 3\end{array}$ & $\begin{array}{l}70.36 \% \\
(29.37 \% \\
\text { bone loss) }\end{array}$ & $70.36 \%$ & - & 0.05 \\
\hline
\end{tabular}


Figure 1. PRISMA flow diagram of the study selection process.
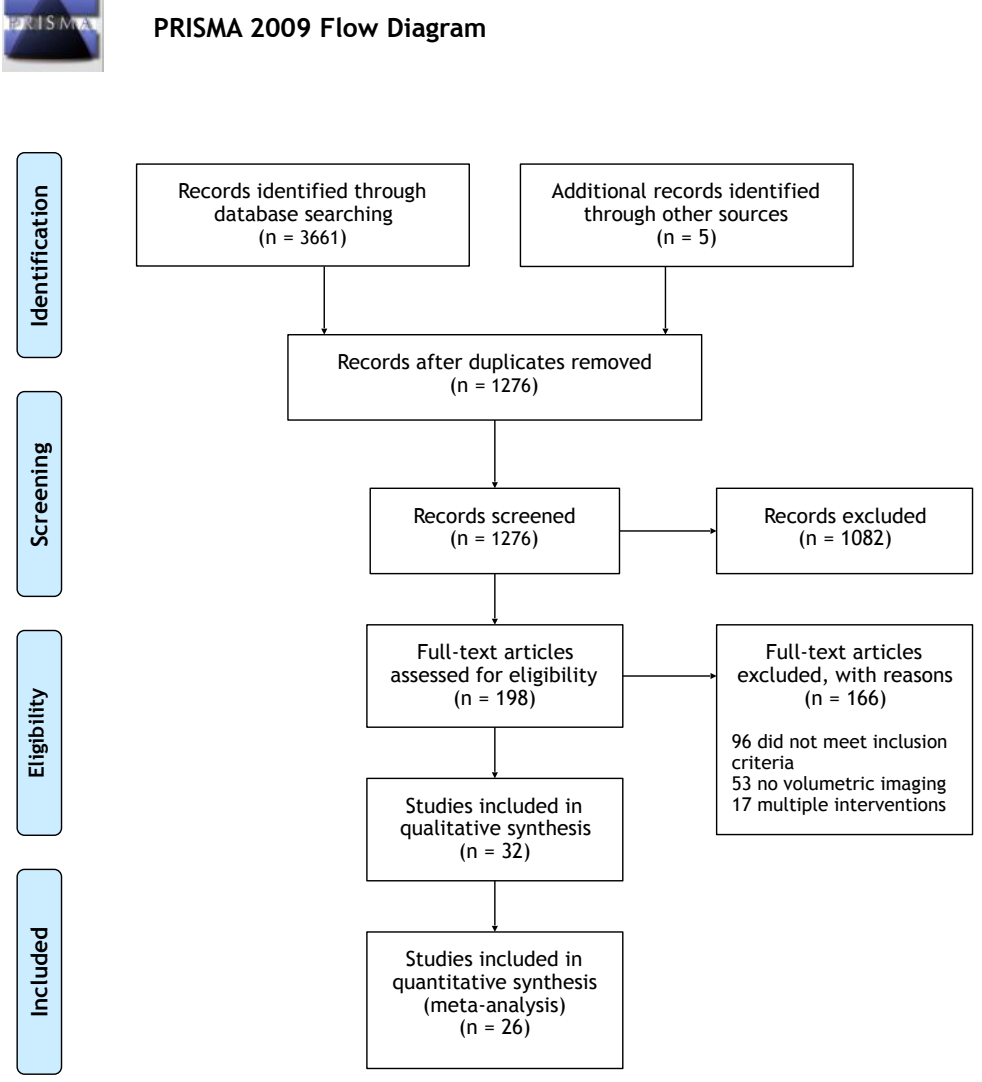

From: Moher D, Liberati A, Tetzlaff J, Altman DG, The PRISMA Group (2009). Preferred Reporting ftems for Systematic Reviews and Meta-Analyses: The PRISMA Statement. PLoS Med 6(7): e1000097. doi:10.1371/journal.pmed1000097

For more information, visit www.prisma-statement.org.

Similarly, overall analysis of investigations $(n=10)$ that used tissue-engineered bone replacement materials (Table 4 ) demonstrated statistically significant reduction in cleft volume (overall SMD = -1.95, 95\% Cl: -2.64, -1.27, P-value < 0.001, $1^{2}=68.7 \%$; Figure 3). On average, percentage bone filling was estimated to be $68.7 \%$ (95\% Cl: 54.5, 82.8) for studies applying tissue-engineered material for bone grafting. None of the 10 studies was classified as being high risk for methodological bias; however, the observed high heterogeneity $\left(R^{2}=68.7 \%\right)$ was attributable to methodological quality (low risk of bias: SMD $=-2.11,95 \%$ Cl: $-3.23,-0.99, P$-value $<0.001, P^{2}=80.3 \%, 6$ studies; moderate risk of bias: $\mathrm{SMD}=-1.81$, 95\% Cl: $-2.40,-1.22, P$-value $<0.001,12=00.0 \%, 4$ studies). 
Table S3 Overview Of The Quality Assessment And Estimated Risk Of Bias In The Studies.

\begin{tabular}{|c|c|c|c|c|c|c|c|c|}
\hline ID & Authors & Year & $\begin{array}{l}\text { Random } \\
\text { Selection } \\
\text { in } \\
\text { Population }\end{array}$ & $\begin{array}{l}\text { Defined } \\
\text { Inclusion } \\
\text { and } \\
\text { Exclusion } \\
\text { Criteria }\end{array}$ & $\begin{array}{l}\text { Loss to } \\
\text { Follow- } \\
\text { Up }\end{array}$ & $\begin{array}{l}\text { Validated } \\
\text { Measure- } \\
\text { ments }\end{array}$ & $\begin{array}{l}\text { Statistical } \\
\text { Analysis }\end{array}$ & $\begin{array}{l}\text { Estimated } \\
\text { Risk of Bias }\end{array}$ \\
\hline 1 & Alonso et al. & 2010 & + & + & + & + & + & Low \\
\hline 2 & Canan Jr et al. & 2012 & + & + & + & + & + & Low \\
\hline 3 & de Ruiter et al. & 2015 & - & + & + & + & + & Moderate \\
\hline 4 & Dickinson et al. & 2008 & + & + & + & + & + & Low \\
\hline 5 & Dieleman et al. & 2004 & - & + & + & + & - & High \\
\hline 6 & Dissaux et al. & 2016 & + & + & + & + & + & Moderate \\
\hline 7 & Feichtinger et al. & 2007 & - & + & - & + & + & High \\
\hline 8 & Feichtinger et al. & 2008 & - & + & - & + & + & High \\
\hline 9 & Herford et al. & 2007 & - & + & + & + & + & Moderate \\
\hline 10 & Honma et al. & 1999 & - & + & - & + & + & High \\
\hline 11 & Hudak et al. & 2014 & - & + & - & + & + & High \\
\hline 12 & Hussain & 2013 & - & + & - & + & + & High \\
\hline 13 & Linderup et al. & 2016 & - & + & - & + & + & High \\
\hline 14 & Nagashima et al. & 2014 & - & + & - & + & + & High \\
\hline 15 & Neovius et al. & 2013 & + & + & + & + & + & Low \\
\hline 16 & Oberoi et al. & 2009 & - & + & + & + & + & Moderate \\
\hline 17 & Oyama et al. & 2004 & - & + & + & + & + & Moderate \\
\hline 18 & Ozawa et al. & 2007 & - & + & - & + & + & High \\
\hline 19 & Pradel et al. & 2012 & - & + & + & + & + & Moderate \\
\hline 20 & Reddy et al. & 2015 & - & + & + & + & + & Moderate \\
\hline 21 & Rychlik et al. & 2012 & - & + & - & + & + & High \\
\hline 22 & Shawky et al. & 2015 & + & + & + & + & + & Low \\
\hline 23 & Tai et al. & 2000 & - & + & + & + & + & Moderate \\
\hline 24 & Takemaru et al. & 2016 & + & + & + & + & + & Low \\
\hline 25 & Touzet-Roumaz. et al. & 2015 & - & + & - & + & + & High \\
\hline 26 & Zhang et al. & 2012 & - & + & + & + & + & Moderate \\
\hline
\end{tabular}


Table 3. Overview of the Analysed Articles in Autogenous Bone Graft group.

\begin{tabular}{|c|c|c|c|c|}
\hline Author (Date) & Type & Graft and donor site & Number of patients & $\%$ Bone fill \\
\hline Alonso et al. (2010) & $\mathrm{RCT}$ & iliac crest & 8 & $80.2 \%$ \\
\hline Canan et al. (2012) & $\mathrm{RCT}$ & iliac crest & 6 & $78 \%$ \\
\hline Dickinson et al. (2008) & $\mathrm{RCT}$ & iliac crest & 12 & $63 \%$ \\
\hline Dieleman et al. (2004) & Prospective & iliac crest & 5 & $75 \%$ \\
\hline Dissaux et al. (2016) & Retrospective & iliac crest & 28 & $54.75 \%$ \\
\hline Feichtinger et al. (2007) & Prospective & iliac crest & 24 & $50.21 \%$ \\
\hline Feichtinger et al. (2008) & Prospective & iliac crest & 20 & $50 \%$ \\
\hline Herford et al. (2007) & Retrospective & iliac crest & 2 & $78.1 \%$ \\
\hline Honma et al. (1999) & Retrospective & iliac crest & 15 & $99 \%$ \\
\hline Hudak et al. (2014) & Retrospective & calvarium & 10 & $85 \%$ \\
\hline Hussain (2013) & Retrospective & Tibia & 9 & $73.15 \%$ \\
\hline Linderup et al. (2016) & Retrospective & Mandibular Smphysis & 32 & $87 \%$ \\
\hline Nagashima et al. (2014) & Retrospective & iliac crest & 29 & $48.28 \%$ \\
\hline Neovius et al. (2013) & $\mathrm{RCT}$ & iliac crest & 3 & $48.67 \%$ \\
\hline Oberoi et al. (2009) & Prospective & iliac crest & 21 & $84 \%$ \\
\hline Oyama et al. (2004) & Retrospective & iliac crest & 5 & $63.67 \%$ \\
\hline Ozawa et al. (2007) & Retrospective & iliac crest & 25 & $53.28 \%$ \\
\hline Pradel et al. (2012) & Prospective & iliac crest & 4 & $36.6 \%$ \\
\hline Reddy et al. (2015) & Prospective & iliac crest & 10 & $67.83 \%$ \\
\hline Rychlik et al. (2012) & Retrospective & iliac crest & 62 & $26 \%$ \\
\hline Shawky et al. (2015) & $\mathrm{RCT}$ & iliac crest & 12 & $68.38 \%$ \\
\hline Tai et al. (2000) & Prospective & iliac crest & 14 & $56.9 \%$ \\
\hline Takemaru et al. (2016) & $\mathrm{RCT}$ & iliac crest & 10 & $59.6 \%$ \\
\hline Touzet-Roumazeille et al. (2015) & Retrospective & iliac crest & 37 & $61.89 \%$ \\
\hline Zhang et al. (2012) & Prospective & iliac crest & 19 & $70.36 \%$ \\
\hline
\end{tabular}

Moreover, results of analysis testing whether the estimated pooled effect sizes (i.e., SMDs) are different across studies using autogenous bone grafts (overall SMD = -1.91) and investigations using tissue-engineered material (overall SMD = -1.95) showed that there is no statistically significant difference between the two major intervention groups ( $P$-value $=$ 0.901). Hence, indicating that both treatment approaches yield similar bone filling capabilities.

In subgroups analysis, studies were grouped based on similarities of the material used to fill the clefts. There were four treatment groups within the studies which used autogenous bone (Table 5) and three treatment groups within the studies which used tissue-engineered materials (Table 6). Results of this analysis showed that all treatment subgroups statistically significantly reduced the cleft volume. In regard to studies using autogenous bone grafts, there was a statistically significant difference in the magnitude of cleft reduc- 
tion (i.e., SMDs) across the treatment subgroups ( $p$-value $=0.009$; Table 5). This difference was mainly driven by a statistically significant difference $(P$-value $=0.004)$ between studies using iliac crest (SMD $=-1.78,95 \% \mathrm{Cl}:-2.11,-1.45)$ and a study using mandibular symphyseal grafts (SMD $=-3.12,95 \% \mathrm{Cl}$ : $-3.95,-2.28$; Table 5). Similarly, reduction in cleft volume was more pronounced, though not statistically significant ( $P$-value $=0.069$ ), in a study using cranial bone grafts (SMD $=-3.22,95 \% \mathrm{Cl}$ : $-4.75,-1.70)$ compared to studies using iliac crest bone). In contrast, studies using different tissue-engineered materials did not differ in regard to cleft filling capability ( $P$-value $=0.362$, Table 6$)$.

Figure 2. Forest plot of random effects meta-analysis summarizing the standardized mean difference of the studies with regard to reduction in postoperative cleft volume using autogenous bone grafts.

\begin{tabular}{|c|c|c|c|c|c|c|c|}
\hline Author (year) & $\begin{array}{c}\text { Hedges' } g \\
\text { SMD }\end{array}$ & $\begin{array}{r}95 \% \\
\text { Lower }\end{array}$ & $\begin{array}{l}6 \mathrm{Cl} \\
\text { Upper }\end{array}$ & $P$-value & Weight \% & Hedges' $g$ SMD and $95 \% \mathrm{Cl}$ & \\
\hline Alonso et al. (2010) & -2.54 & -3.94 & -1.15 & 0.000 & 2.95 & & \\
\hline Canan et al. (2012) & -2.19 & -3.60 & -0.78 & 0.002 & 2.91 & & \\
\hline Dickinson et al. (2008) & -3.64 & -5.18 & -2.09 & 0.000 & 2.64 & & \\
\hline Dieleman et al. (2004) & -0.75 & -1.59 & 0.09 & 0.081 & 4.33 & & \\
\hline Dissaux et al. (2016) & -2.59 & -3.35 & -1.82 & 0.000 & 4.53 & & \\
\hline Feichtinger et al. (2007) & -1.22 & -1.74 & -0.70 & 0.000 & 5.19 & & \\
\hline Feichtinger et al. (2008) & -1.60 & -2.25 & -0.95 & 0.000 & 4.85 & & \\
\hline Herford et al. (2007) & -1.95 & -3.65 & -0.26 & 0.024 & 2.38 & & \\
\hline Homma et al. (1999) & -2.59 & -3.63 & -1.55 & 0.000 & 3.78 & & \\
\hline Hudak et al. (2014) & -3.22 & -4.75 & -1.70 & 0.000 & 2.69 & & \\
\hline Hussain (2013) & -2.46 & -3.74 & -1.18 & 0.000 & 3.20 & & \\
\hline Linderup et al. (2016) & -3.12 & -3.95 & -2.28 & 0.000 & 4.34 & & \\
\hline Nagashima et al. (2014) & -1.50 & -2.02 & -0.97 & 0.000 & 5.18 & & \\
\hline Neovius et al. (2013) & -0.91 & -1.88 & 0.06 & 0.067 & 3.97 & & \\
\hline Oberoi et al. (2009) & -2.08 & -2.83 & -1.33 & 0.000 & 4.57 & & \\
\hline Oyama et al. (2004) & -1.87 & -3.23 & -0.52 & 0.007 & 3.03 & & \\
\hline Ozawa et al. (2007) & -1.73 & -2.35 & -1.12 & 0.000 & 4.95 & & \\
\hline Pradel et al. (2012) & -0.78 & -1.68 & 0.11 & 0.087 & 4.18 & & \\
\hline Reddy et al. (2015) & -1.04 & -1.77 & -0.31 & 0.005 & 4.64 & & \\
\hline Rychlik et al. (2012) & -0.72 & -0.99 & -0.44 & 0.000 & 5.69 & & \\
\hline Shawky et al. (2015) & -2.25 & -3.29 & -1.21 & 0.000 & 3.78 & & \\
\hline Tai et al. (2000) & -1.93 & -2.80 & -1.06 & 0.000 & 4.25 & & \\
\hline Takemaru et al. (2016) & -2.47 & -3.70 & -1.25 & 0.000 & 3.33 & & \\
\hline Touzet-Roumazeille et al. (2015) & -1.91 & -2.45 & -1.38 & 0.000 & 5.14 & & \\
\hline Zhang et al. (2012) & -3.38 & -4.54 & -2.22 & 0.000 & 3.49 & & \\
\hline Overall (Random effects) & -1.91 & -2.25 & -1.57 & 0.000 & & & \\
\hline \multicolumn{7}{|c|}{ Heterogeneity: $\mathrm{Q}$-value $=105.7, \mathrm{df}=24, P$-value $<0.001 ; P^{2}=77.3 \%$} & 6.00 \\
\hline
\end{tabular}

Results show stałjstically significant large reduction in cleft volume (overall SMD $=-1.91,95 \% \mathrm{Cl}:-2.25,-1.57$, $P$-value $<0.001,1=77.3 \%)$.

In an additional analysis, we tested whether cleft volume reduction is different across studies using iliac crest grafts (SMD = -1.78; Table 5) and studies using growth factors (SMD $=-2.34$; Table 6). Results of this analysis did not show any statistically significant difference between these two major subgroups ( $P$-value $=0.324)$. 
Table 4 Overview of the Analysed Articles in Tissue-Engineered Bone Replacement group.

\begin{tabular}{|c|c|c|c|c|}
\hline Author (Date) & Type & Graft and donor site & $\begin{array}{l}\text { Number of } \\
\text { patients }\end{array}$ & \% Bone fil \\
\hline Alonso et al. (2010) & $\mathrm{RCT}$ & $\begin{array}{l}\text { resorbable collagen matrix with rhBMP-2 } \\
\text { (Infuse Bone Graft) }\end{array}$ & 8 & $74.4 \%$ \\
\hline Canan et al. (2012) & $\mathrm{RCT}$ & $\begin{array}{l}\text { resorbable collagen sponge with } \\
\text { rhBMP-2 (Infuse Bone Graft) }\end{array}$ & 6 & $75.1 \%$ \\
\hline de Ruiter et al. (2015) & Prospective & $\begin{array}{l}\text { micro-structured beta-TCP mixes with } \\
\text { autologous blood ( } 65 \% \text { porous and }>90 \% \\
\text { pure phase B-TCP) }\end{array}$ & 6 & $73 \%$ \\
\hline Dickinson et al. (2008) & $\mathrm{RCT}$ & $\begin{array}{l}\text { resorbable collagen matrix with rhBMP-2 } \\
\text { (Infuse Bone Graft) }\end{array}$ & 9 & $95 \%$ \\
\hline Herford et al. (2007) & Retrospective & $\begin{array}{l}\text { resorbable type I bovine collagen matrix } \\
\text { with rhBMP-2 (Infuse Bone Graft) }\end{array}$ & 10 & $71.7 \%$ \\
\hline Neovius et al. (2013) & $\mathrm{RCT}$ & BMP-2 in hydrogel & 4 & $46 \%$ \\
\hline Oyama et al. (2004) & Retrospective & ICBG + PRP + Fibrin glue & 7 & $80.19 \%$ \\
\hline Pradel et al. (2012) & Prospective & $\begin{array}{l}\text { autogenous Osteoblasts cultured on } \\
\text { demineralised bone matrix Osteovit }\end{array}$ & 4 & $40.9 \%$ \\
\hline Shawky et al. (2015) & $\mathrm{RCT}$ & $\begin{array}{l}\text { PRF (Platelet rich fibrin) + conventional } \\
\text { iliac crest bone graft }\end{array}$ & 12 & $82.61 \%$ \\
\hline Takemaru et al. (2016) & $\mathrm{RCT}$ & $\begin{array}{l}\text { bioabsorbable hydroxyapatite and } \\
\text { collagen complex (0.5 ml HA/Col) }\end{array}$ & 5 & $73.3 \%$ \\
\hline
\end{tabular}

Figure 3. Forest plot of random effects meta-analysis summarizing the standardized mean difference of the studies with regard to reduction in postoperative cleft volume using tissue-engineered bone substitute materials.

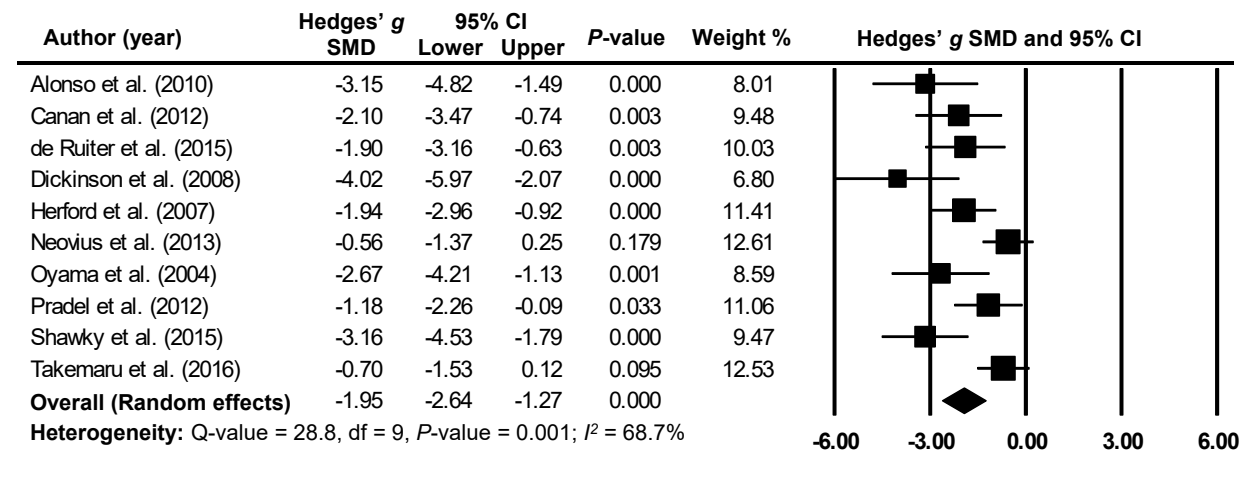

Results słow statistically significant reduction in cleft volume (overall SMD $=-1.95,95 \% \mathrm{Cl}:-2.64,-1.27, P$-value $<0.001,1=68.7 \%)$. 
Table 5 Subgroup analysis of studies using autogenous bone graft: results of random effects meta-analysis.

\begin{tabular}{|c|c|c|c|c|c|}
\hline Bone graft material & $\begin{array}{c}\text { Number of } \\
\text { Studies }\end{array}$ & $\begin{array}{l}\text { Number } \\
\text { of Subjects }\end{array}$ & $\begin{array}{c}\text { Hedges' g SMD* } \\
(95 \% \mathrm{Cl})\end{array}$ & SE & $\begin{array}{c}\text { Within group } \\
P \text {-value }\end{array}$ \\
\hline Iliac crest & 22 & 371 & $-1.78(-2.11,-1.45)$ & 0.169 & $<0.001$ \\
\hline Mandibular symphyseal & 1 & 32 & $-3.12(-3.95,-2.28)$ & 0.426 & $<0.001$ \\
\hline Cranial & 1 & 10 & $-3.22(-4.75,-1.70)$ & 0.777 & $<0.001$ \\
\hline Tibial & 1 & 9 & $-2.46(-3.74,-1.18)$ & 0.654 & $<0.001$ \\
\hline Between groups $P$-value ${ }^{\ddagger}$ & & & 0.009 & & \\
\hline
\end{tabular}

SMD: Standardized mean difference; SE: Standard error; Cl: Confidence interval

* Refers to the standardized difference in mean cleft volume pre- and post-operatively.

${ }^{\dagger}$ Testing whether the treatment statistically significantly reduced cleft volume.

₹ Testing whether there is a statistically significant difference in SMD between the four treatment groups.

Table 6 Subgroup analysis of studies using tissue-engineered bone replacement materials: results of random effects meta-analysis.

\begin{tabular}{|c|c|c|c|c|c|}
\hline Bone graft material & $\begin{array}{l}\text { Number } \\
\text { of Studies }\end{array}$ & $\begin{array}{c}\text { Number } \\
\text { of Subjects }\end{array}$ & $\begin{array}{l}\text { Hedges' } g \text { SMD* } \\
(95 \% \mathrm{CI})\end{array}$ & SE & $\begin{array}{c}\text { Within group } \\
P_{\text {-value }}^{\dagger}\end{array}$ \\
\hline Growth factors & 6 & 49 & $-2.34(-3.39,-1.28)$ & 0.540 & $<0.001$ \\
\hline Enhanced scaffolds and cell therapy & 2 & 11 & $-1.82(-3.27,-0.37)$ & 0.742 & 0.014 \\
\hline $\begin{array}{l}\text { Bio-composites and hemostatic } \\
\text { agents }\end{array}$ & 2 & 11 & $-1.20(-2.35,-0.05)$ & 0.587 & 0.041 \\
\hline Between groups $P$-value & & & 0.362 & & \\
\hline \multicolumn{6}{|c|}{$\begin{array}{l}\text { SMD: Standardized mean difference; SE: Standard error; Cl: Confidence interval } \\
{ }^{*} \text { Refers to the standardized difference in mean cleft volume pre- and post-operatively. } \\
{ }^{\dagger} \text { Testing whether the treatment statistically significantly reduced cleft volume. }\end{array}$} \\
\hline
\end{tabular}

\section{Publication bias}

Funnel plots were used to assess the presence of publication bias. Among both treatment groups, small studies reporting small effect sizes (negative studies) are missing (Figure S1 and Figure S2). Moreover, there is a lack of large studies reporting the effectiveness of tissue-engineered materials. 
Chapter 2

Figure S1. Funnel plot of standard error by standardized difference in mean in studies using autogenous bone grafts after removing the two outlier studies.

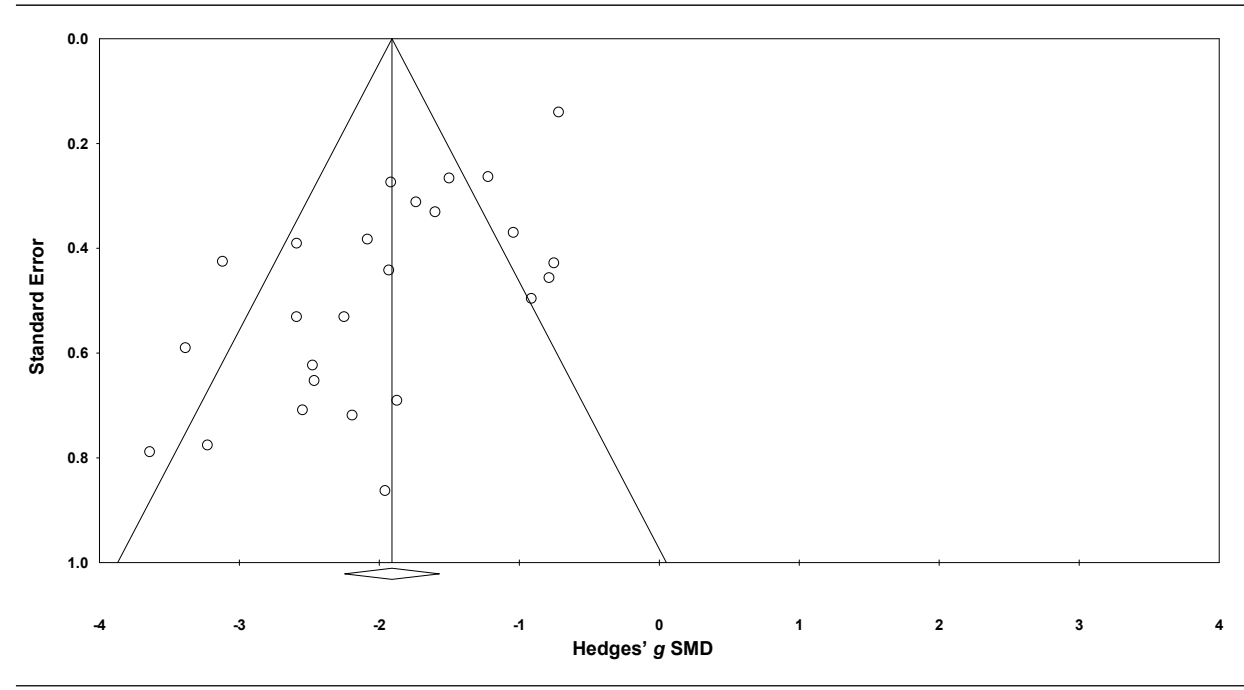

Figure S2. Funnel plot of standard error by standardized difference in mean in studies using tissue-engineered bone substitute materials after removing the outlier study.

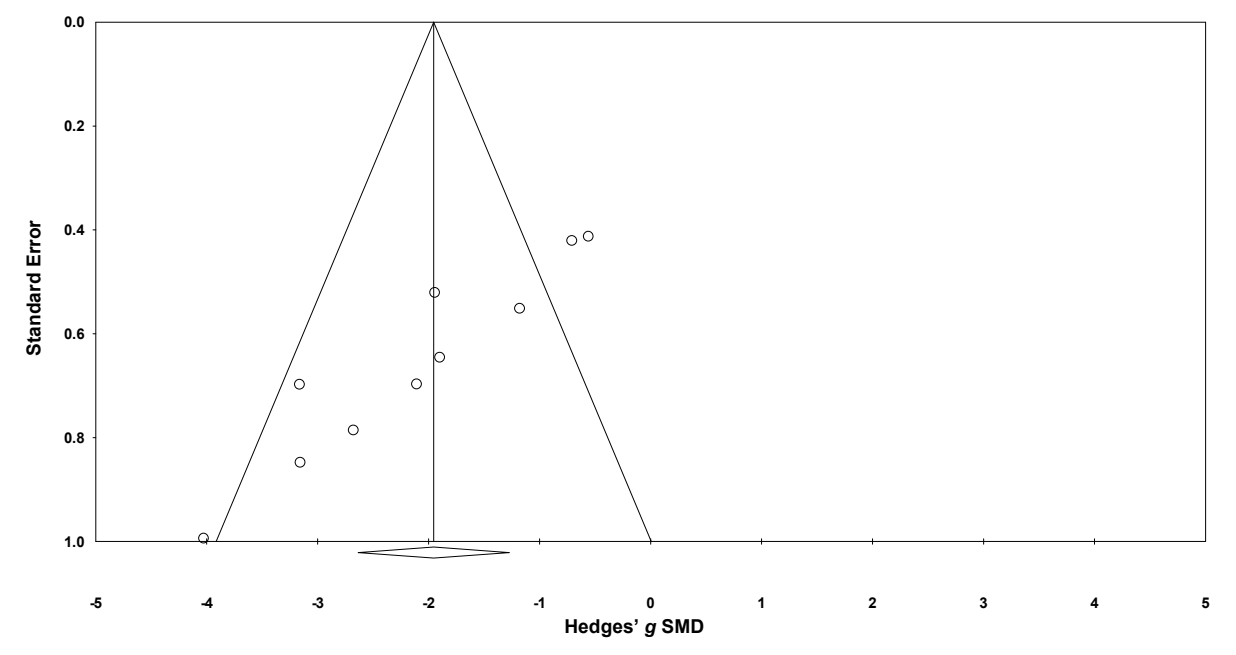




\section{DISCUSSION}

Autogenous bone most commonly harvested from the iliac crest remains to be the standard of care to graft alveolar cleft deformities, yet alternative sites have also been used such as the calvaria, tibia, and mandibular symphysis. 3, 7, 8, 10 However, an autogenous graft is associated with significant drawbacks inclulding limited supply of bone, the need for an additional donor site and its associated postoperative morbidities such as pain, hematoma, and delayed ambulation, and its inherent susceptibility to resorption in the long term. 3, 7, 8, 10, 50,51 For this reason, several tissue-engineered bone substitutes have been used as alternative materials to promote bone formation and eliminate donor site morbidities. ${ }^{4-9}$

Despite the presence of multiple systematic reviews in the literature evaluating alveolar bone grafting interventions, the literature lacks a meta-analysis of volumetric 3D outcome of these materials. ${ }^{7,8,52,53}$ Previous 2D assessment of grafting outcome using autogenous bone according to the Bergland grading system reported success rates ranging from $67 \%$ to $95 \%$. ${ }^{13,54}$ In recent years, computed tomography (CT) scans have been widely utilized for the outcome assessment of secondary bone grafting procedures in cleft patients. Some studies reported that conventional 2D assessment of grafting procedures have overestimated the number of successful bone grafts by $17 \%$ based on dental radiographs compared to computed tomography, which was can be explained by the loss of dimensional assessment related to depth and overlying structures. ${ }^{13,15,55}$

Results of this comprehensive meta-analysis suggest that both, autogenous bone (average percentage bone fill $=62.0 \%$ ) and tissue-engineered bone substitutes (average percentage bone fill $=68.7 \%)$, yielded similar effectiveness in filling alveolar clefts ( $P$-value $=0.901)$. The tissue-engineering materials utilized expressed a considerable amount of heterogeneity. The majority of the regenerative materials were growth factors such as BMP-2, likely because of their longer presence in clinical practice compared to novel scaffolds and cell therapy. A previous meta-analysis of BMP-2 by Kelly et al reported significant increase in bone height in localized alveolar ridge augmentation in contrast to the control group with an overall SMD of 0.56 (Cl, 0.20-0.92) in favor of BMP. ${ }^{56}$ In addition, a network meta-analysis by Papageorgiou et al comparing various grafts histomorphically prior to dental implantation showed no significant differences in the percentage of new bone formation between synthetic bone and autogenous bone, with synthetic substitute group showing percentage new bone of 5.5\% lower than autogenous bone. ${ }^{57}$

Another meta-analysis by Klijn compared various donor sites of autogenous bone in maxillary sinus floor augmentation et al histomorphometrically and showed that graft harvested 
from the iliac crest resulted in a significantly lower total bone volume when compared with intraoral donor sites, namely the mandibular symphysis and ramus. ${ }^{58}$

This coincides with our results, which show standardized difference in mean cleft volume of $-1.78(-2.11,-1.45)$ for the autogenous iliac crest graft, which is lower than the mandibular symphysis, -3.12 (-3.95, -2.28), cranium, -3.22 (-4.75, -1.70), and tibia grafts, $-2.46(-3.74$, -1.18). Additional studies in the literature reported that iliac crest bone graft is inherently prone to resorption of up to $43.1 \%$ in the first year after grafting. ${ }^{8,50,51}$

The majority of the studies used autogenous bone obtained from the iliac crest (22 studies) in contrast to calvarial, tibial, and mandibular symphysis grafts. This is understandable given it is still the gold standard and the large amount of bone it provides. Our literature review suggests a lack of good quality RCTs evaluating alveolar cleft repair. This meta-analysis provides evidence that regenerative bone substitute materials show promising results in alveolar cleft repair.

\section{CONCLUSIONS}

Our meta-analysis results indicate that tissue-engineered bone substitutes are as effective as autogenous bone in reducing cleft volume creating the viable option of eliminating the need for a second surgical site its associated postoperative morbidities.

\section{DISCLOSURE}

The authors have no financial interest regarding the content of this article.

\section{CONFLICTS OF INTEREST}

None.

\section{FUNDING}

This research did not receive any specific grant from funding agencies in the public, commercial, or not-for-profit sectors. 


\section{REFERENCES}

1. Rychlik D, Wójcicki P, Koźlik M. Osteoplasty of the alveolar cleft defect. Adv Clin Exp Med2012. p. 255-62.

2. Santiago PE, Schuster LA, Levy-Bercowski D. Management of the alveolar cleft. Clin Plast Surg2014. p. 219-32.

3. Seifeldin SA. Is alveolar cleft reconstruction still controversial? (Review of literature). Saudi Dent J2016. p. 3-11.

4. Chung VH, Chen AY, Jeng LB, Kwan CC, Cheng SH, Chang SC. Engineered autologous bone marrow mesenchymal stem cells: alternative to cleft alveolar bone graft surgery. The Journal of craniofacial surgery. 2012;23:1558-63.

5. Gładysz D, Hozyasz KK. Stem cell regenerative therapy in alveolar cleft reconstruction. Arch Oral Biol2015. p. 1517-32.

6. Kawata T, Kohno S, Fujita T, Sugiyama H, Tokimasa C, Kaku M, et al. New biomaterials and methods for craniofacial bone defect: chondroid bone grafts in maxillary alveolar clefts. Journal of craniofacial genetics and developmental biology. 2000;20:49-52.

7. Janssen NG, Weijs WL, Koole R, Rosenberg AJ, Meijer GJ. Tissue engineering strategies for alveolar cleft reconstruction: a systematic review of the literature. Clinical oral investigations. 2014;18:219-26.

8. Khojasteh A, Kheiri L, Motamedian SR, Nadjmi N. Regenerative medicine in the treatment of alveolar cleft defect: A systematic review of the literature. Journal of cranio-maxillo-facial surgery: official publication of the European Association for Cranio-Maxillo-Facial Surgery2015. p. 1608-13.

9. KolkA, Handschel J, Drescher W, Rothamel D, Kloss F, Blessmann M, et al. Current trends and future perspectives of bone substitute materials - from space holders to innovative biomaterials. Journal of cranio-maxillo-facial surgery : official publication of the European Association for Cranio-Maxillo-Facial Surgery. 2012;40:706-18.

10. Ma'amon AR, Telfah $\mathrm{H}$. Secondary alveolar bone grafting: the dilemma of donor site selection and morbidity. British Journal of Oral and Maxillofacial Surgery. 2008;46:665-70.

11. Sharif F, Rehman IU, Muhammad N, MacNeil S. Dental materials for cleft palate repair. Materials Science and Engineering: C. 2016;61:1018-28.

12. Bergland $\mathrm{O}$, Semb G, Abyholm FE. Elimination of the residual alveolar cleft by secondary bone grafting and subsequent orthodontic treatment. The Cleft palate journal. 1986;23:175-205.

13. Oberoi S, Chigurupati R, Gill P, Hoffman WY, Vargervik K. Volumetric Assessment of Secondary Alveolar Bone Grafting Using Cone Beam Computed Tomography. The Cleft Palate-Craniofacial Journal. 2009;46:503-11.

14. Waitzman AA, Posnick JC, Armstrong DC, Pron GE. Craniofacial skeletal measurements based on computed tomography: Part II. Normal values and growth trends. The Cleft Palate-Craniofacial Journal. 1992;29:118-28.

15. Meij vd, J A, Baart, A J, Prahl-Andersen, B, et al. Bone volume after secondary bone grafting in unilateral and bilateral clefts determined by computed tomography scans. Oral surgery, oral medicine, oral pathology, oral radiology, and endodontics. 2001;92:136-41.

16. Moher D, Liberati A, Tetzlaff J, Altman DG, Group P. Preferred reporting items for systematic reviews and meta-analyses: the PRISMA statement. PLoS med. 2009;6:e1000097.

17. Al-Moraissi EA, Ellis E. Surgical treatment of adult mandibular condylar fractures provides better outcomes than closed treatment: a systematic review and meta-analysis. Journal of Oral and Maxillofacial Surgery. 2015;73:482-93.

18. Liberati A, Altman DG, Tetzlaff J, Mulrow C, Gøtzsche PC, loannidis JP, et al. The PRISMA statement for reporting systematic reviews and meta-analyses of studies that evaluate health care interventions: explanation and elaboration. PLoS Med. 2009;6:e1000100.

19. Stroup DF, Berlin JA, Morton SC, Olkin I, Williamson GD, Rennie D, et al. Meta-analysis of observational studies in epidemiology: a proposal for reporting. Jama. 2000;283:2008-12.

20. Vandenbroucke JP, Von Elm E, Altman DG, Gøtzsche PC, Mulrow CD, Pocock SJ, et al. Strengthening the Reporting of Observational Studies in Epidemiology (STROBE): explanation and elaboration. PLoS Med. 2007;4:e297. 
21. Borenstein M, Hedges LV, Higgins JPT, Rothstein HR. Introduction to Meta-Analysis: Wiley; 2009.

22. Higgins JP, Green S. Cochrane handbook for systematic reviews of interventions: John Wiley \& Sons; 2011.

23. Higgins J, Thompson SG, Deeks J], Altman DG. Measuring inconsistency in meta-analyses [journal article as teaching resource, deposited by John Flynn]. British medical journal. 2003;327:557-60.

24. Hudak K, Hettinger P, Denny A. Cranial bone grafting for alveolar clefts: A 25-year review of outcomes. Cleft Palate-Craniofacial Journal2014. p. e38.

25. Alonso; N; Tanikawa; Y DFS, R; Canan Jr; L; Ozawa; O, T; Rocha; L, D. Evaluation of maxillary alveolar reconstruction using a resorbable collagen sponge with recombinant human bone morphogenetic protein-2 in cleft lip and palate patients. Tissue Eng Part C Methods2010. p. 1183-9.

26. Canan Jr LWdSF, Renato; Alonso, Nivaldo; Tanikawa, Daniela Yukie Sakai; Rocha, Diógenes Laércio; Coelho, Julio Cezar Uili. Human Bone Morphogenetic Protein-2 Use for Maxillary Reconstruction in Cleft Lip and Palate Patients. The Journal of craniofacial surgery2012. p. 1627-33.

27. de Ruiter AJ, Nard; van Es, Robert; Frank, Michael; Meijer, Gert; Koole, Ron; Rosenberg, Toine. Micro-structured Beta-Tricalcium Phosphate for Repair of the Alveolar Cleft in Cleft Lip and Palate Patients: A Pilot Study. The Cleft Palate-Craniofacial Journal2015. p. 336-40.

28. Dickinson BPA, Rebekah K; Wasson, Kristy L; O'Hara, Catherine; Gabbay, Joubin; Heller, Justin B; Bradley, James P. Reduced Morbidity and Improved Healing with Bone Morphogenic Protein-2 in Older Patients with Alveolar Cleft Defects. Plast Reconstr Surg2008. p. 209-17.

29. Dieleman FJ, Tump P, Baart JA, Tuinzing DB, Bezemer PD. A computed tomographic evaluation of change in bone volume after secondary bone grafting over the first postoperative year. Plastic and reconstructive surgery. 2004;114:738-42.

30. Dissaux CB, Frédéric; Grollemund, Bruno; Bridonneau, Thomas; Kauffmann, Isabelle; Mattern, Jean-François; Bruant-Rodier, Catherine. Evaluation of success of alveolar cleft bone graft performed at 5 years versus 10 years of age. Journal of Cranio-Maxillofacial Surgery2016. p. 21-6.

31. Feichtinger MZ, Wolfgang; Mossböck, Rudolf; Kärcher, Hans. Three-dimensional evaluation of secondary alveolar bone grafting using a 3D- navigation system based on computed tomography: a two-year follow-up. $\mathrm{Br}$ J Oral Maxillofac Surg2008. p. 278-82.

32. Feichtinger M, Mossbock R, Karcher $\mathrm{H}$. Assessment of bone resorption after secondary alveolar bone grafting using three-dimensional computed tomography: a three-year study. Cleft Palate Craniofac J2007. p. 142-8.

33. Herford ASB, Philip J; Rawson, Rick; Williams, Roland P. Bone Morphogenetic Protein-Induced Repair of the Premaxillary Cleft. Journal of Oral and Maxillofacial Surgery2007. p. 2136-41.

34. Honma KK, Tadaharu; Nakajima, Tamio; Hayasi, Takafumi. Computed tomographic evaluation of bone formation after secondary bone grafting of alveolar clefts. Journal of Oral and Maxillofacial Surgery1999. p. 1209-13.

35. Hussain S. Evaluation of alveolar grafting with tibial graft in adolescent patients. Indian J Dent Res2013. p. 659

36. Linderup BWC, Paolo M; Jensen, John; Küseler, Annelise. Mandibular Symphyseal Bone Graft for Reconstruction of Alveolar Cleft Defects: Volumetric Assessment With Cone Beam Computed Tomography 1-Year Postsurgery. The Cleft Palate-Craniofacial Journal2016. p. 64-72.

37. Nagashima HS, Yoshiaki; Ogata, Hisao; Miyamoto, Junpei; Yazawa, Masaki; Kishi, Kazuo. Evaluation of Bone Volume After Secondary Bone Grafting in Unilateral Alveolar Cleft Using Computer-Aided Engineering. The Cleft Palate-Craniofacial Journal2014. p. 665-8.

38. Neovius; E; Lemberger; M; Skogh DC, A; Hilborn; J; Engstrand; T. Alveolar bone healing accompanied by severe swelling in cleft children treated with bone morphogenetic protein-2 delivered by hydrogel. J Plast Reconstr Aesthet Surg2013. p. 37-42.

39. Oberoi SC, Radhika; Gill, Pawandeep; Hoffman, William Y; Vargervik, Karin. Volumetric Assessment of Secondary Alveolar Bone Grafting Using Cone Beam Computed Tomography. The Cleft Palate-Craniofacial Journal2009. p. 503-11.

40. Oyama TN, Soh; Tsugawa, Tomoe; Shimizu, Fumiaki. Efficacy of platelet-rich plasma in alveolar bone grafting. Journal of Oral and Maxillofacial Surgery2004. p. 555-8. 
41. Ozawa TO, Susumu; Fukuyama, Eiji; Matsui, Yoshiro; Torikai, Katuyuki; Fujita, Kiyohide. Factors Influencing Secondary Alveolar Bone Grafting in Cleft Lip and Palate Patients: Prospective Analysis Using CT Image Analyzer. The Cleft Palate-Craniofacial Journal2007. p. 286-91.

42. Pradel WL, Günter. Tissue-engineered bone grafts for osteoplasty in patients with cleft alveolus. Annals of Anatomy - Anatomischer Anzeiger2012. p. 545-8.

43. Reddy; G MBR, V; Rao; E, V; Chaitanya; J, J; Allareddy; S; C, C. Prospective analysis of secondary alveolar bone grafting in cleft lip and palate patients. Journal of international oral health : JIOH2015. p. 73-7.

44. Rychlik DW, Piotr. Bone Graft Healing in Alveolar Osteoplasty in Patients With Unilateral Lip, Alveolar Process, and Palate Clefts. The Journal of craniofacial surgery2012. p. 118-23.

45. Shawky; H; Seifeldin; A S. Does Platelet-Rich Fibrin Enhance Bone Quality and Quantity of Alveolar Cleft Reconstruction? Cleft Palate Craniofac J2015.

46. Tai C-CES, I Scott; McFadden, Leland. Prospective analysis of secondary alveolar bone grafting using computed tomography. Journal of Oral and Maxillofacial Surgery2000. p. 1241-9.

47. Takemaru MS, Yoshiaki; Sakamoto, Teruo; Kishi, Kazuo. Assessment of bioabsorbable hydroxyapatite for secondary bone grafting in unilateral alveolar cleft. Journal of Plastic, Reconstructive \&amp; Aesthetic Surgery2016. p. 493-6.

48. Touzet-Roumazeille SV-F, Brigitte; Kadlub, Natacha; Genin, Michaël; Dissaux, Caroline; Raoul, Gwenaël; Ferri, Joël; Vazquez, Marie-Paule; Picard, Arnaud. Osseous and dental outcomes of primary gingivoperiosteoplasty with iliac bone graft: A radiological evaluation. Journal of Cranio-Maxillofacial Surgery2015. p. 950-5.

49. Zhang W, Shen G, Wang X, Yu H, Fan L. Evaluation of alveolar bone grafting using limited cone beam computed tomography. Oral surgery, oral medicine, oral pathology and oral radiology. 2012;113:542-8.

50. Le BT, Woo I. Alveolar cleft repair in adults using guided bone regeneration with mineralized allograft for dental implant site development: a report of 2 cases. Journal of Oral and Maxillofacial Surgery. 2009;67:1716-22.

51. Schultze-Mosgau S, Nkenke E, Schlegel AK, Hirschfelder U, Wiltfang J. Analysis of bone resorption after secondary alveolar cleft bone grafts before and after canine eruption in connection with orthodontic gap closure or prosthodontic treatment. Journal of oral and maxillofacial surgery. 2003;61:1245-8.

52. Guo J, Li C, Zhang Q, Wu G, Deacon SA, Chen J, et al. Secondary bone grafting for alveolar cleft in children with cleft lip or cleft lip and palate. The Cochrane Library. 2011.

53. van Hout WM, van der Molen ABM, Breugem CC, Koole R, Van Cann EM. Reconstruction of the alveolar cleft: can growth factor-aided tissue engineering replace autologous bone grafting? A literature review and systematic review of results obtained with bone morphogenetic protein-2. Clinical Oral Investigations. 2011;15:297-303.

54. da Silva Filho OG, Teles SG, Ozawa TO, Filho LC. Secondary bone graft and eruption of the permanent canine in patients with alveolar clefts: literature review and case report. The Angle Orthodontist. 2000;70:174-8.

55. Lee C, Crepeau RJ, Williams HB, Schwartz S. Alveolar Cleft Bone Grafts: Results andImprecisions of the Dental Radiograph. Plastic and reconstructive surgery. 1995;96:1534-8.

56. Kelly MP, Vaughn OLA, Anderson PA. Systematic review and meta-analysis of recombinant human bone morphogenetic protein-2 in localized alveolar ridge and maxillary sinus augmentation. Journal of Oral and Maxillofacial Surgery. 2016;74:928-39.

57. Papageorgiou SN, Papageorgiou PN, Deschner J, Götz W. Comparative effectiveness of natural and synthetic bone grafts in oral and maxillofacial surgery prior to insertion of dental implants: Systematic review and network meta-analysis of parallel and cluster randomized controlled trials. Journal of dentistry. 2016;48:1-8.

58. Klijn RJ, Meijer GJ, Bronkhorst EM, Jansen JA. Sinus floor augmentation surgery using autologous bone grafts from various donor sites: a meta-analysis of the total bone volume. Tissue Engineering Part B: Reviews. 2010;16:295-303. 


$$
3
$$




\section{"Comparison of Bone Grafts from Various Donor Sites in Human Bone Specimens"}

Mohammad Kamal; Felix Gremse; Stefanie Rosenhain;

Alexander K. Bartella; Frank Hölzle; Peter Kessler; Bernd Lethaus

Published in the Journal of Craniofacial Surgery. 2018 May 14. doi 10.1097/SCS.0000000000004586 


\section{ABSTRACT}

The objective of the current study was to compare the three-dimensional morphometric microstructure in human cadaveric bone specimens taken from various commonly utilized donor sites for autogenous bone grafting. Autogenous bone grafts can be harvested from various anatomical sites and express heterogeneous bone quality with a specific three-dimensional microstructure for each site. The long-term structural integrity and susceptibility to resorption of the graft depend on the selected donor bone. Micro-computed tomography generates high-resolution datasets of bone structures and calcifications making this modality versatile for microarchitecture analysis and quantification of the bone. Six bone specimens, $10 \mathrm{~mm}$ in length, where anatomically possible, were obtained from various anatomical sites from 10 human dentate cadavers ( 4 men, 6 women, mean age 69.5 years). Specimens were scanned using a Micro-computed tomography device and volumetrically reconstructed. A virtual cylindrical inclusion was reconstructed to analyze the bone mineral density and structural morphometric analysis using bone indices: relative bone volume, surface density, trabecular thicknesses, and trabecular separation. Calvarial bone specimens showed the highest mineral density, followed by the chin, then mandibular ramus then the tibia, whereas iliac crest and maxillary tuberosity had lower bone mineral densities. The pairwise comparison revealed statistically significant differences in the bone mineral density and relative bone volume index in the calvaria, mandibular ramus, mandibular symphysis groups when compared to those in the iliac crest and maxillary tuberosity, suggesting higher bone quality in the former groups than in the latter; tibial specimens expressed variable results. 


\section{INTRODUCTION}

Bone grafting is a widely-used procedure for the reconstruction of bony defects in the craniofacial region. Bone augmentation procedures aim primarily at expanding the volume of viable bone along with the establishment of a functional skeletal biological medium which allows for an optimal physiologic integration and predictable healing. Autogenous bone grafts from intramembranous or endochondral embryonic origins can be harvested from various donor sites, each offering heterogeneous bone quality and distinctive biomechanical features and structural integrity making them selectively prone to resorption and loss of graft volume over time. ${ }^{1-5}$ Thus, the long-term structural integrity and tissue quality of the grafted bone depends on the type of the grafting material and the autogenous bone donor site. ${ }^{2,3}$ Additional considerations must be taken into account when planning for a bone grafting procedure from various intraoral and distant donor sites. These include the limited bone supply at each distinct anatomical region, subsequent donor site morbidity and its effect on the patient's quality of living, the reliability of the anatomical landmarks, and most importantly patient's acceptance and satisfaction about the procedure. ${ }^{1-3,5,6}$

Micro-computed tomography $(\mu \mathrm{CT})$ is a modern imaging modality which is used to generate three-dimensional (3D) high-resolution data sets of the in-vivo laboratory animals or excised organs. 7,8 The volumetric $\mu \mathrm{CT}$ data is suitable for visualization and quantitative image analysis due to the isotropic voxel sizes and well-calibrated voxel units. ${ }^{9}$ Bone structures and calcifications provide a particularly strong native contrast which make this modality versatile for the analyze the microarchitecture of the skeletal tissue and to quantify bone, while analysis of blood vessels requires the application of contrast agents. 10, 11 Various morphometric structural features have been reported in the literature to influence the augmentation ability of a specific bone type, including grafted bone particle sizes, porosity, biomechanical stress capacity, plasticity, and cortical bone content. 2,3,12,13

We sought to compare the skeletal morphometric microstructure of human cadaveric bone specimens taken from the commonly utilized donor sites for autogenous bone grafting ( $\mathrm{ABG}$ ) using $\mathrm{MCT}$. Images obtained are analyzed after surface rendering of the bone specimen's microstructure and quantification of the bone mineral density along with bone quality parameters. 


\section{MATERIALS AND METHODS}

\section{Human subjects and bone specimens}

Human cadavers from 10 Caucasian subjects were obtained for the study at the Institute of Anatomy, RWTH University in Aachen, Germany. Collection of these human tissue specimens was conducted by strictly following the protocol established by the Human Ethics Committee of Aachen University Hospital. Date of birth of each subject was provided, but no data were available regarding previous medical diseases, prior medical or surgical interventions, or the cause of death of these individuals. Specimen were obtained from dentate cadavers (4 males, 6 females). The age ranged from 57.3 to 81.2 years (mean 69.5 years). A trephine bur of $8 \mathrm{~mm}$ internal diameter was employed to harvest bone cores at multiple intraoral and distant extraoral donor sites from anatomical areas routinely utilized in non-vascularized bone grafting procedures in oral and maxillofacial surgery. These anatomical donor sites were: calvarium (CA), maxillary tuberosity (MT), mandibular ramus (MR), mandibular symphysis (MS), anterior iliac crest (IC), and tibia (TB). One bone specimen, $10 \mathrm{~mm}$ in length where anatomy permitted, was obtained from each of the six anatomic locations for each of 10 cadavers (10 specimens from each anatomic location). The bone specimens were thoroughly cleansed of any soft tissue material and immersed in $0.16 \%$ chlorhexidine solution and subsequently dried. A total of 60 edentulous bone block specimens from various skeletal regions were obtained and prepared for imaging.

\section{Acquisition of $\mu C T$ scans and image analysis}

Specimens were scanned using a $\mathrm{CCT}$ device (Tomoscope DUO, CT-Imaging, Erlangen, Germany), as previously described. ${ }^{14}$ The dual energy $\mu \mathrm{CT}$ acquired 720 projections with $1032 \times 1012$ pixels for each of the two detectors during one full rotation. Scanning time was $90 \mathrm{~s}$. Volumetric data was reconstructed at $35 \mu \mathrm{m}$ isotropic voxel size using a Feldkamp algorithm. The voxel intensities were calibrated for Hounsfield units using a water phantom. A virtual cylindrical inclusion with a 4-mm diameter was inserted into the center of the bone specimen using the Imalytics Preclinical Software (Philips Technologie GmbH, Aachen, Germany) to analyze the bone mineral density. ${ }^{9,14}$ Then, quantitative bone quality parameters were computed for the entire cylindrical inclusion as well as along the z-axis of the inclusion. Specimen results from each respective location in all ten cadavers were pooled together. Mean values and standard deviations of all the recorded parameters were statistically compared across all groups.

\section{Assessment of micro-architecture and bone quality parameters and statistical analysis}

The 3D structural images produced by the analytical software were used to obtain the results of the morphometric analysis. These structural bone quality parameters included: bone mineral density (BMD) presented as kHU bone volume fraction (BV/TV), which rep- 
resents the ratio of volume of binary objects inside a volume of interest (VOI) to total volume (TV) of VOI expressed as percentage; object surface to volume ratio (BS/BV) in $\mathrm{mm}^{-1}$, which represents the ratio of surface area of binary objects to volume of these objects to characterize the thickness and complexity of a 3D structure; trabecular thickness (Tb.Th) in mm, which represents the local thickness of an individual trabecula in a 3D-structure; and trabecular separation (Tb.Sp) in mm, which represents the local thickness of voids in in a porous structure. ${ }^{15,16}$

Statistical comparison was performed using one-way-ANOVA with Tukey posttest. Statistical pairwise significances among different types of bone grafts were displayed as a visual matrix (green indicated statistical significance $p<0.05$ ). 


\section{RESULTS}

\section{Comparison of 3D morphometric parameters among the anatomical sites}

Table 1 demonstrates the 3D characteristics of harvested specimens from various regions. Noteworthy is that the lowest amount of bone height was obtained from the maxillary tuberosity specimens. Surface rendering of the bone specimen showed variable bone mineral density (BMD) along the reconstructed virtual cylinder along the bone grafts, as seen in Figure 1. Biphasic pattern of increased BMD was noted in specimens from the $C A, M R$, and MS, and monophasic increased BMD in the TB group. The lowest BMD was noted in the MT and IC groups, as demonstrated in Figure 2 and 3.

Figure 1. CT data and image analysis.
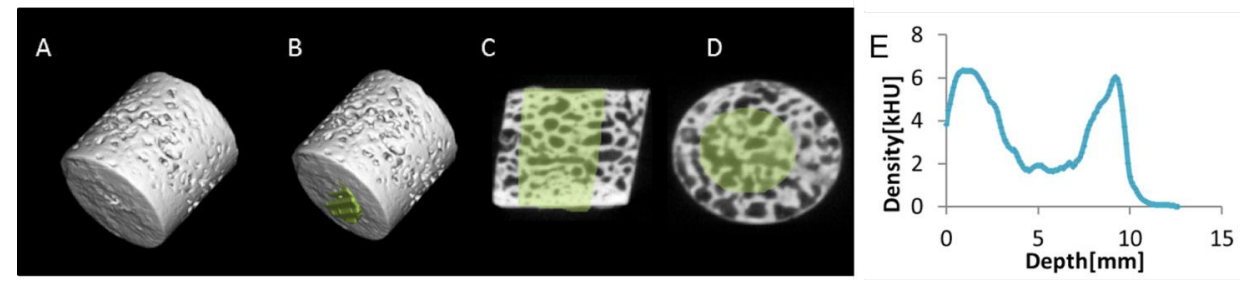

A) Surface rendering of a bone specimen from mandibular symphysis showing the trabecular bone structure. B) A cylindrical region was inserted into the specimen for analysis. C) Represents a longitudinal view of the rendered slice through the center of the specimen whereas D) represents a cross-sectional view, showing lower bone mineral density in the center. E) Bone mineral density along the cylinder.

Table 1. Characteristics of harvested specimens from various anatomical positions.

\begin{tabular}{lccc}
\hline Bone Specimen Site & Number of Specimens $(\mathrm{n})$ & Width & Average height (mean and SD) \\
\hline Calvarium (Parietal) & 10 & $8 \mathrm{~mm}$ & $7.22 \mathrm{~mm} \pm 1.57$ \\
Maxillary Tuberosity & 10 & $8 \mathrm{~mm}$ & $4.86 \mathrm{~mm} \pm 2.05$ \\
Mandibular Ramus & 10 & $8 \mathrm{~mm}$ & $7.6 \mathrm{~mm} \pm 3.33$ \\
Mandibular Symphysis (Chin) & 10 & $8 \mathrm{~mm}$ & $9.8 \mathrm{~mm} \pm 3.35$ \\
Iliac Crest (Anterior) & 10 & $8 \mathrm{~mm}$ & $11.85 \mathrm{~mm} \pm 3.34$ \\
Tibia (Lateral Tubercle) & 10 & $8 \mathrm{~mm}$ & $11.2 \mathrm{~mm} \pm 4.22$ \\
\hline
\end{tabular}

Table 2 demonstrates the morphometric bone parameters (mean and standard deviations) of the specimens from various anatomical positions. When assessing cancellous bone mass parameters, we noticed that CA, MS, and MR specimens expressed significantly higher BV/TV values than MT and IC specimens. The TB specimens expressed a wider range of values with slightly improved values over the IC and MT, and were significantly different from CA specimens. Tb.Sp values yielded significantly lower trabecular separation 
values in the CA group than in the MT, IC, and TB groups, and in the MS group than in the MT group (Figure 4). The findings of the structural indices overall suggest more favorable values representing BMD and BV/TV mainly in the CA group, followed by MS, then MR group than in the TB, MT, and IC groups (Figure 4).

Figure 2. Bone mineral density of different specimens.
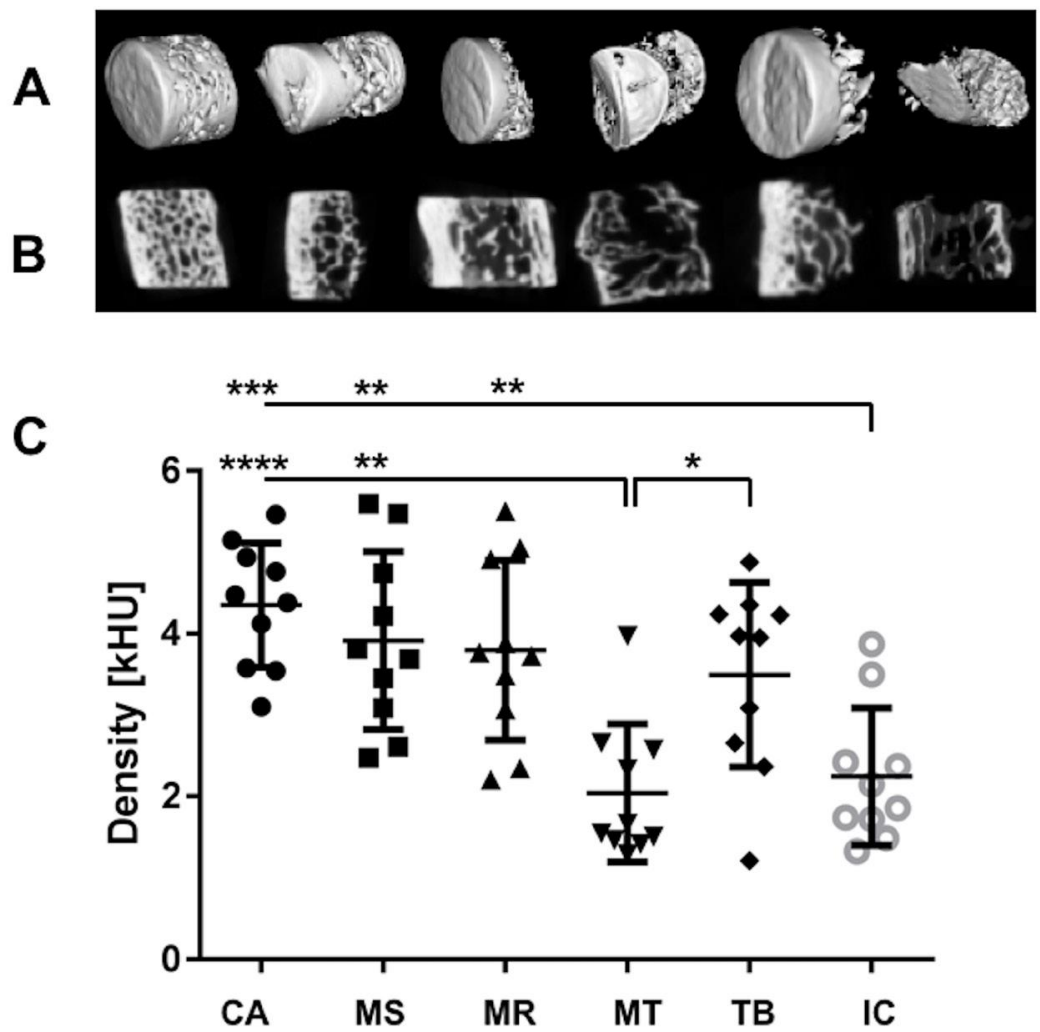

A) Surface rendering of representative samples; left-to-right: CA: calvarial, MT: maxillary tuberosity, MR: mandibular ramus, MS: mandibular symphysis, IC: iliac crest, TB: tibia. B) CT slices through the representative samples. C) Bone mineral densities quantified by $\mu C T$ in means and standard deviations. Statistical comparison was performed using one-way-ANOVA with Tukey posttest. The pairwise significances are visualized as bars among groups (* $p \leq 0.05$; ** $p \leq 0.01$; *** $p \leq 0.001$ ), i.e., CA vs. MT, CA vs. IC, MS vs. MT, MS vs. IC, MR vs. MT, MR vs. IC, and MT vs. TB were significantly different.

Figure 5 shows post hoc pairwise significances $(p<0.05)$ as a visualized matrix between the various groups. Bones from CA, MS, and MR demonstrated significantly higher BMD when compared to those of bones from IC and MT (Figure 5). Notable is that BMD values from 
CA, MS, and MR groups were not statistically different from those from TB, making it higher than IC and MT. Trabecular thickness (Tb.Th) values were largely non-different between groups, except between MT and TB. However, trabecular separation (Tb.Sp) values were significant when comparing CA to the MT, IC and TB, in addition to MS to MT (Figure 5).

Table 2. Morphometric bone parameters of specimens from various anatomical positions (mean \pm standard deviation)

\begin{tabular}{lccccc}
\hline & $B M D(\mathrm{kHU})$ & BV/TV $(\%)$ & $B S / B V\left(\mathrm{~mm}^{-1}\right)$ & Tb.Th $(\mathrm{mm})$ & Tb.Sp $(\mathrm{mm})$ \\
\hline Calvarium & $4.35 \pm 0.76$ & $71.59 \pm 13.60$ & $106.63 \pm 40.99$ & $0.023 \pm 0.010$ & $0.015 \pm 0.006$ \\
Mandibular Symphysis & $3.91 \pm 1.09$ & $48.19 \pm 23.22$ & $188.09 \pm 136.85$ & $0.022 \pm 0.011$ & $0.023 \pm 0.006$ \\
Iliac Crest & $2.25 \pm 0.84$ & $16.74 \pm 13.08$ & $1290.55 \pm 1152.69$ & $0.011 \pm 0.009$ & $0.029 \pm 0.008$ \\
Mandibular Ramus & $3.80 \pm 1.10$ & $40.28 \pm 11.81$ & $187.16 \pm 105.22$ & $0.024 \pm 0.009$ & $0.028 \pm 0.005$ \\
Maxillary Tuberosity & $2.04 \pm 0.85$ & $23.39 \pm 7.97$ & $245.18 \pm 106.10$ & $0.012 \pm 0.007$ & $0.038 \pm 0.019$ \\
Tibia & $3.49 \pm 1.13$ & $36.53 \pm 19.54$ & $431.31 \pm 446.83$ & $0.027 \pm 0.019$ & $0.032 \pm 0.008$ \\
\hline
\end{tabular}

Figure 3. Bone mineral density curves.
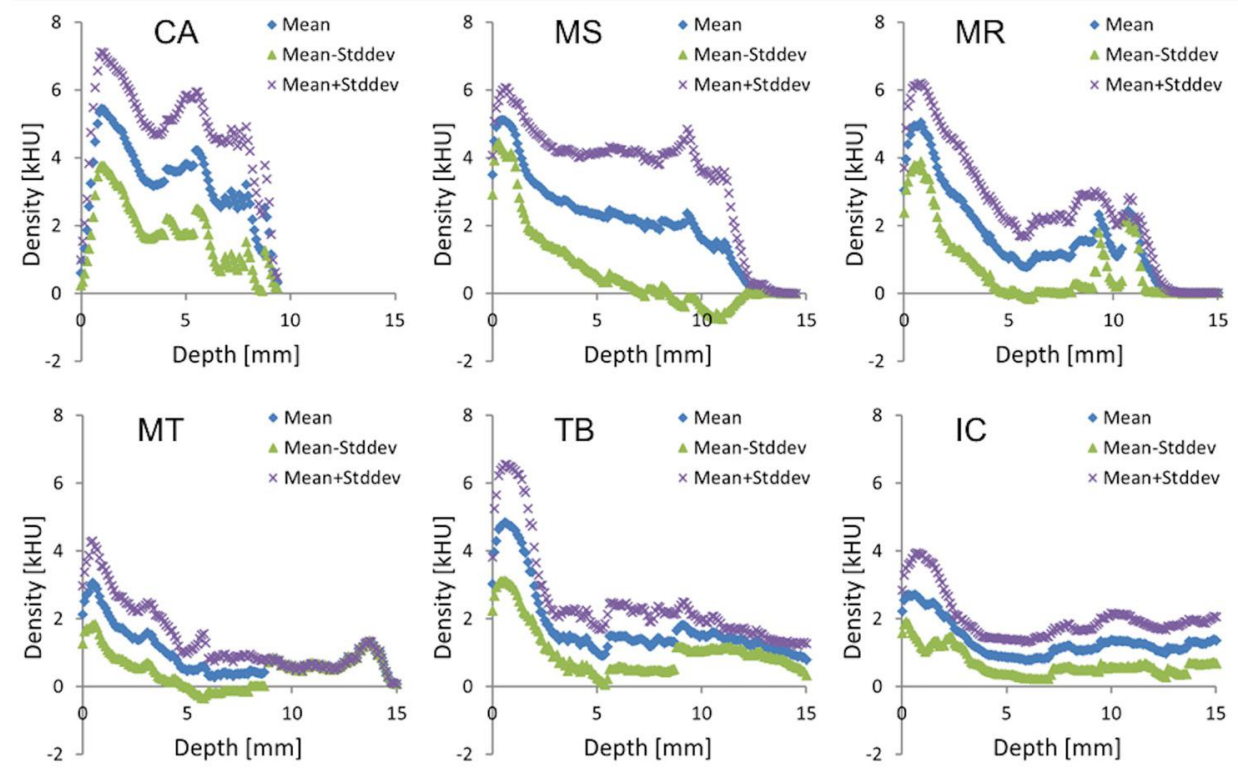

Density curves along the z-axis were determined for all 10 specimens of each kind. The mean curves are shown in blue together with curves for mean + standard deviation and mean-standard deviation. 
Figure 4. Morphometric bone parameters of specimens from various anatomical positions.
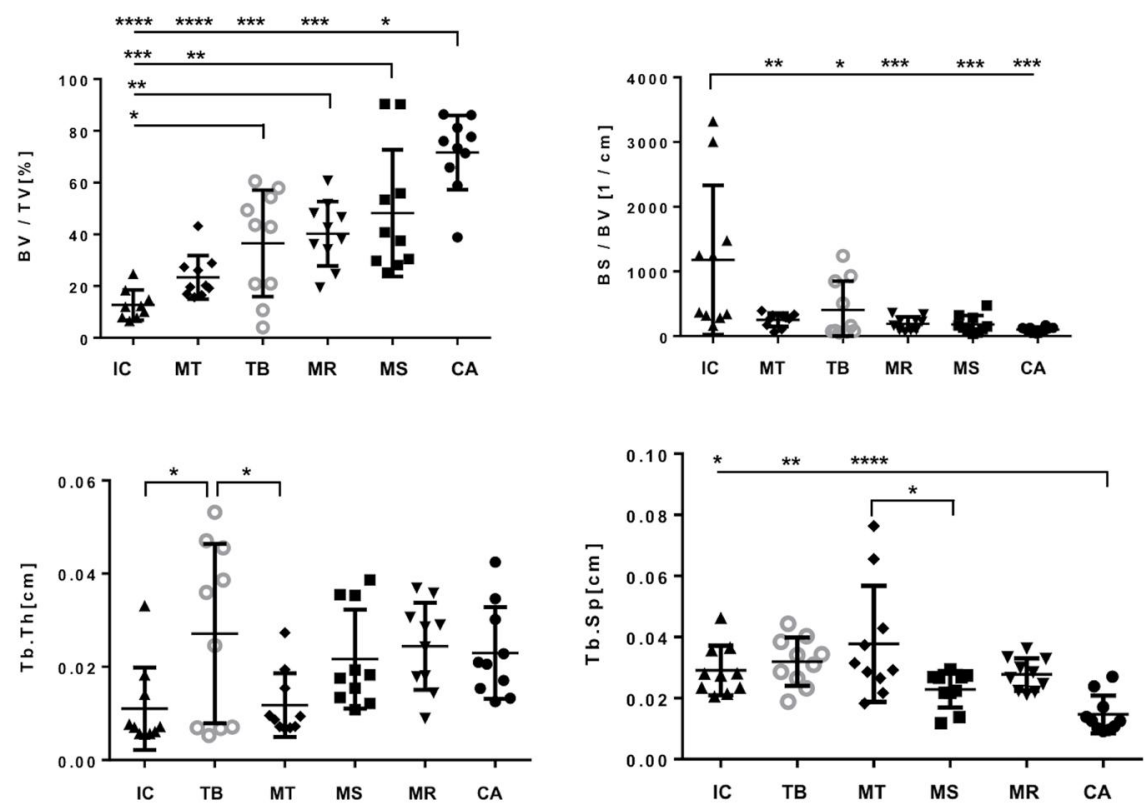

Statistical comparison was performed using one-way-ANOVA with Tukey posttest. The pairwise significances are visualized as bars (* $p<0.05 ;$ ** $p<0.01$; ** $p<0.001$ ). 
Figure 5. Pairwise significances.

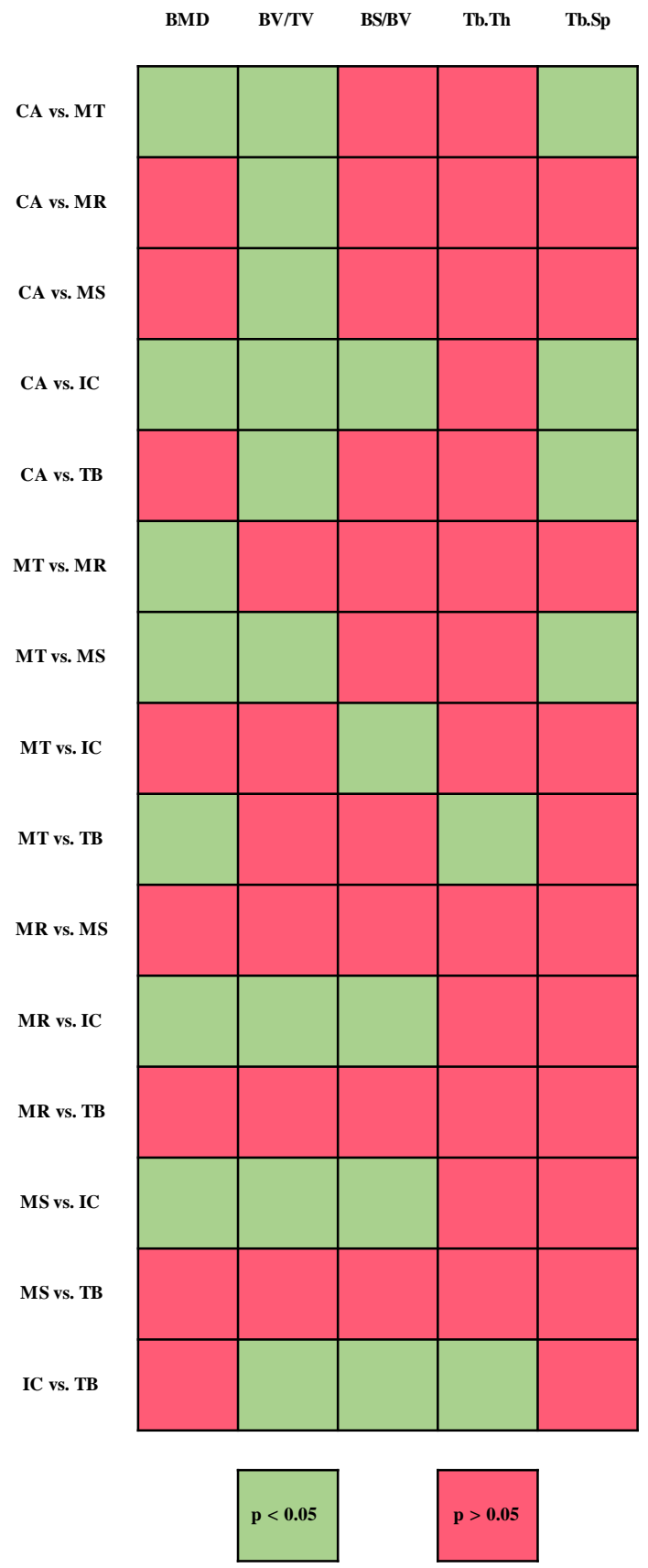

Statistical comparison was performed using one-way-ANOVA with Tukey posttest. The pairwise significances are visualized as a matrix (green means $p \leq 0.05$ ). 


\section{DISCUSSION}

Despite recent technological advances in the development of bone substitute materials for utilization in bone grafting, conventional autogenous non-vascularized bone obtained from several anatomical donor areas remains to be the current gold standard for grafting many types of bone defects in the craniofacial region. ${ }^{3}$ The selection of the proper autogenous bone grafting donor site is thus of high clinical significance and surgeons require careful recognition of the properties offered by each graft type to achieve maximal level of structural stability and desirable wound healing. ${ }^{1-5}$ Structural bone qualities (macro- and micro-architecture) along with the biomaterial characteristics such as bone mineral density represent the mechanical strength of bone, and commonly evaluated as the main predictor of bone quality in bone grafting. 2,3,12,17,18 Current advancement in imaging technology lead to significant improvement of imaging resolution of skeletal structural architecture in vivo and in ex vivo structures. $\mathrm{MCT}$ has particularly added a deeper insight into evaluating calcified tissue and been used in the quantitative analysis bone formation in human and animal research to evaluate bone graft healing, in contrast to conventional histology which reveals more on the cellular properties of the calcified tissue. 12, 15, 19 In addition to bone mineral density, several morphometric parameters have been proposed to assess the mechanical characteristics of bone quality using 3D $\mu \mathrm{CT}$ images. ${ }^{12,15,16}$

Despite that most previous studies in the field on dental implantology have highlighted the role of cortical bone in mechanical bone strength, given its physical stiffness, leading to achieving early primary stabilization during implant insertion, several recent studies have also evaluated the effect of the 3D spatial architecture of trabecular bone on the overall biomechanical strength and stress-bearing characteristic of bone. ${ }^{12,13,15,18,20}$ Further studies evaluated the qualitative and quantitative structural morphology for evaluating bone grafts and regenerative bone substitute materials during healing bone healing and integration of the grafts to answer questions related to the heterogeneous bone graft resorption and maintenance of bone volume between intramembranous and endochondral bone. 21, 22 Previous studies have found that bone grafts volume maintenance was affected by the dynamic microstructural interactions, along with local grafting site mechanical factors like intact periosteum, mechanical fixation of the graft, and similarity between grafted and recipient bone to allow graft incorporation and controlled rate of resorption. ${ }^{21-23}$ Vinci et. al. evaluated calvarial bone grafts morphologically using $\mu C T$ to assess their biomechanical and healing properties and demonstrated full graft incorporation, enhanced bone density, and dense trabecular connections similar in structure to native bone with 38.50\% BV/TV. ${ }^{21}$ In an in-vivo study by Lu and Rabie evaluating bone healing of endochondral versus intramembranous bone grafts in mandibular defects in rabbits using $\mu \mathrm{CT}$, they demonstrated enhanced bone volume at 55.3\% with intramembranous bone grafts compared with endochondral 
bone $(P<0.0001)$ with improved maintenance of graft volume and graft resorption rate of 16.2\% compared to 38.3\% in the endochondral group. ${ }^{24}$ Another study by Kim and Henkin investigated the bone quality from maxilla and mandible in 34 human cadavers by using $\mu C T$ to evaluate BMD and BV/TV. Their results showed a wide spectrum of density across the maxillary and mandibular bone specimens, with all mandibular morphometric values exceeding the maxillary values by 3.3 fold representing superior bone quality. ${ }^{25}$

In this study, we analyzed 60 human cadaveric bone specimens sampled from donor sites commonly used in ABG procedures with $\mu \mathrm{CT}$ for BMD, trabecular morphology and architecture. Our results show great variation in bone densities and 3D morphometric values across different donor sites. Parameters evaluating bone quality were highest with the calvarial bone, followed by the mandibular symphysis, mandibular ramus, tibia, and least in the maxillary tuberosity and anterior iliac crest as seen in the pairwise significance test (Figure 2, 4, 5). With regard to BMD, our data demonstrated biphasic morphology of the bony microarchitecture representing compact bone relative to the trabecular bone as evaluated from the superficial bone surface toward the bone marrow, with notably increased biphasic BMD in the specimens from the calvarium, mandibular ramus, and mandibular synthesis, and increased monophasic BMD in the tibia group, with the lowest BMD noted in the maxillary tuberosity and iliac crest groups (Figure 3). Our results show that bone grafts obtained from these sites are likely to be more compact, bicortical in nature with higher mineral density value and overall better trabecular bone quality parameters. This seems to be consistent with several reports in the literature reporting that bone obtained from these sites tend to demonstrate better bone quality, retain their volume longer, and are less prone to resorption over time. 1, 2,5,21,24 Our results also demonstrated that cancellous bone mass assessment through BV/TV index yielded higher values for the CA, MS and MR groups than for the MT and IC, while trabecular separation values (Tb.Sp) were significantly lower for the CA group than for the tuberosity, IC, and TB groups and for the MS values than for the MT (Figure 4). (Table 2, Figure 4). This significant difference in BMD, cancellous bone mass, and microarchitecture structural indices may be responsible for the lower rate of graft volume maintenance and subsequent resorption.

We believe that our results highlight the various microstructural properties of various bone grafts commonly used in bone grafting, and may help clinicians in better selection their bone grafts for reconstruction of the craniofacial skeleton. This study provides an insight into the structural differences among various bone graft donor sites. Further research needs to be performed to compare bone tissues in a larger study with more subjects, along with obtaining detailed medical history if possible, to correlate the imaging findings with the medical history and medicinal uptake to better assess the bone qualities and provide a better clinical report. 


\section{ACKNOWLEDGMENT:}

The authors would like to thank Professor Andreas Prescher, Department of Anatomy at RWTH Aachen University, for the supervision of the cadaveric surgery. Mohammad Kamal and Felix Gremse contributed equally to this paper.

\section{CONFLICT OF INTEREST:}

We have no conflicts of interest. 


\section{REFERENCES}

1. Nkenke E, Neukam FW. Autogenous bone harvesting and grafting in advanced jaw resorption: morbidity, resorption and implant survival. Eur J Oral Implantol. 2014;7:S203-17.

2. Kon K, Shiota M, Ozeki M, Yamashita Y, Kasugai S. Bone augmentation ability of autogenous bone graft particles with different sizes: a histological and micro-computed tomography study. Clinical Oral Implants Research: Blackwell Publishing Ltd; 2009. p. 1240-6.

3. Kolk A, Handschel J, Drescher W, Rothamel D, Kloss F, Blessmann M, et al. Current trends and future perspectives of bone substitute materials - from space holders to innovative biomaterials. J Craniomaxillofac Surg2012. p. 706-18.

4. Nauth A, Lane J, Watson JT, Giannoudis P. Bone Graft Substitution and Augmentation. Journal of Orthopaedic Trauma2015. p. S34-S8.

5. Sakkas A, Wilde F, Heufelder M, Winter K, Schramm A. Autogenous bone grafts in oral implantology -is it still a "gold standard"? A consecutive review of 279 patients with 456 clinical procedures. International Journal of Implant Dentistry. 2017;3:23.

6. Jensen AT, Jensen SS, Worsaae N. Complications related to bone augmentation procedures of localized defects in the alveolar ridge. A retrospective clinical study. Oral and maxillofacial surgery. 2016;20:115-22.

7. Ehling J, Babickova J, Gremse F, Klinkhammer BM, Baetke S, Knuechel R, et al. Quantitative Micro-Computed Tomography Imaging of Vascular Dysfunction in Progressive Kidney Diseases. Journal of the American Society of Nephrology : JASN. 2016;27:520-32.

8. Gremse F, Theek B, Kunjachan S, Lederle W, Pardo A, Barth S, et al. Absorption reconstruction improves biodistribution assessment of fluorescent nanoprobes using hybrid fluorescence-mediated tomography. Theranostics. 2014;4:960-71.

9. Gremse F, Stark M, Ehling J, Menzel JR, Lammers T, Kiessling F. Imalytics Preclinical: Interactive Analysis of Biomedical Volume Data. Theranostics. 2016;6:328-41.

10. Ehling J, Bartneck M, Wei X, Gremse F, Fech V, Mockel D, et al. CCL2-dependent infiltrating macrophages promote angiogenesis in progressive liver fibrosis. Gut. 2014;63:1960-71.

11. Gremse F, Grouls C, Palmowski M, Lammers T, de Vries A, Grull H, et al. Virtual elastic sphere processing enables reproducible quantification of vessel stenosis at CT and MR angiography. Radiology. 2011;260:709-17.

12. Kim J-E, Shin J-M, Oh S-O, Yi W-J, Heo M-S, Lee S-S, et al. The three-dimensional microstructure of trabecular bone: analysis of site-specific variation in the human jaw bone. Imaging science in dentistry. 2013;43:227-33.

13. Katranji A, Misch K, Wang H-L. Cortical bone thickness in dentate and edentulous human cadavers. Journal of periodontology. 2007;78:874-8.

14. Gremse F, Doleschel D, Zafarnia S, Babler A, Jahnen-Dechent W, Lammers T, et al. Hybrid microCT-FMT imaging and image analysis. Journal of visualized experiments : JoVE. 2015:e52770.

15. Ulrich D, Van Rietbergen B, Laib A, Ruegsegger P. The ability of three-dimensional structural indices to reflect mechanical aspects of trabecular bone. Bone. 1999;25:55-60.

16. Hildebrand T, Laib A, Müller R, Dequeker J, Rüegsegger P. Direct three-dimensional morphometric analysis of human cancellous bone: microstructural data from spine, femur, iliac crest, and calcaneus. J Bone Miner Res: John Wiley and Sons and The American Society for Bone and Mineral Research (ASBMR); 1999. p. 1167-74.

17. Fanuscu MI, Chang T-L. Three-dimensional morphometric analysis of human cadaver bone: microstructural data from maxilla and mandible. Clinical Oral Implants Research2004. p. 213-8.

18. Pothuaud L, Van Rietbergen B, Mosekilde L, Beuf O, Levitz P, Benhamou CL, et al. Combination of topological parameters and bone volume fraction better predicts the mechanical properties of trabecular bone. Journal of Biomechanics. 2002;35:1091-9. 
19. Lappalainen O-P, Karhula SS, Haapea M, Kauppinen S, Finnilä M, Saarakkala S, et al. Micro-CT Analysis of Bone Healing in Rabbit Calvarial Critical-Sized Defects with Solid Bioactive Glass, Tricalcium Phosphate Granules or Autogenous Bone. Journal of oral \& maxillofacial research. 2016;7.

20. Misch CE, Qu Z, Bidez MW. Mechanical properties of trabecular bone in the human mandible: implications for dental implant treatment planning and surgical placement. Journal of oral and maxillofacial surgery : official journal of the American Association of Oral and Maxillofacial Surgeons. 1999;57:700-6; discussion 6-8.

21. Vinci R, Rebaudi A, Capparè $P$, Gherlone E. Microcomputed and histologic evaluation of calvarial bone grafts: a pilot study in humans. International Journal of Periodontics and Restorative Dentistry. 2011;31:389.

22. Ozaki W, Buchman SR. Volume maintenance of onlay bone grafts in the craniofacial skeleton: micro-architecture versus embryologic origin. Plastic and reconstructive surgery. 1998;102:291-9.

23. Phillips JH, Rahn BA. Fixation effects on membranous and endochondral onlay bone graft revascularization and bone deposition. Plastic and reconstructive surgery. 1990;85:891-7.

24. Lu M, Rabie A. Quantitative assessment of early healing of intramembranous and endochondral autogenous bone grafts using micro-computed tomography and Q-win image analyzer. International journal of oral and maxillofacial surgery. 2004;33:369-76.

25. Kim YJ, Henkin J. Micro-computed tomography assessment of human alveolar bone: bone density and three-dimensional micro-architecture. Clin Implant Dent Relat Res2015. p. 307-13. 


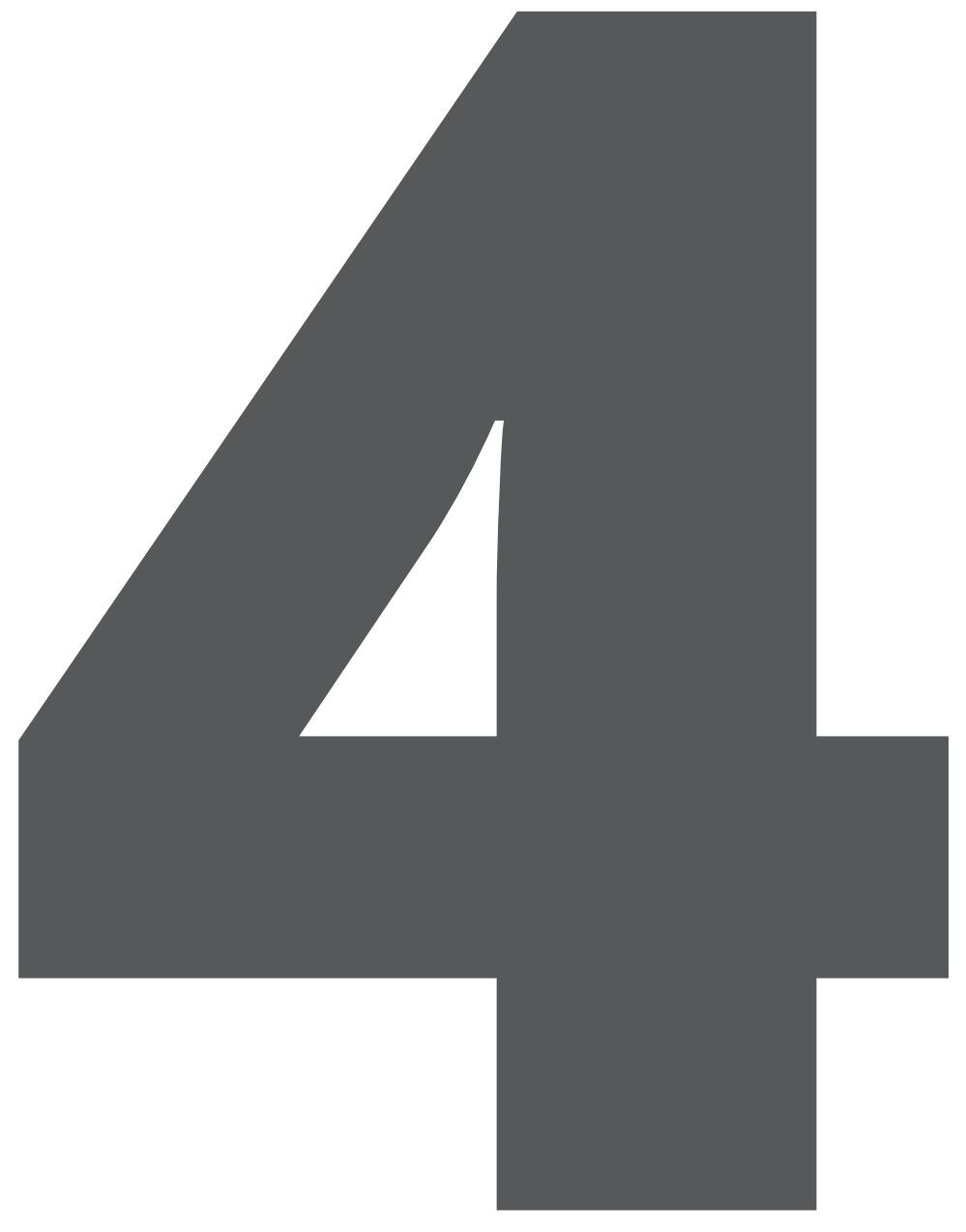




\section{"New Bone Formation Around Xenogenic Dentin Grafts to Rabbit Tibia Marrow"}

Adel Al-Asfour; Lars Andersson; Mohammad Kamal; Bobby Joseph

Published in Dental Traumatology. 2013 Dec; 29:455-460.

doi: 10.1111/edt.12045 


\section{ABSTRACT}

Purpose: From traumatology, it is well known that dentoalveolar ankylosis results in osseous replacement and formation of new bone. This principle is used after decoronation for preservation of the height and width of the alveolar bone crest after trauma. Dentin possesses bone-forming properties and may possibly also be used as a bone augmentation material prior to implant placement. The aim of this study was to investigate whether xenogenic dentin particles inserted into the marrow space of rabbit tibia, a space where there is no solid bone tissue initially, would contribute to new bone formation.

Materials and Methods: Dentin chips from human teeth were inserted into tibias of ten New Zealand rabbits. The tibial bones were processed for histology after 6 months, and new bone formation was quantified.

Results: Bone formation was ranging from 0 to $86 \%$ on the dentin fragments, and there was minor inflammation. Bone formation was seen to a larger extent on dentin grafts located close to the native tibial bone wall. There was a significant correlation ( $r=0.579$, $P<0.001$ ) between the amount of bone formation around the dentin graft and distance to the tibial cortical wall.

Conclusion: Dentin promotes new bone formation when located close to native cortical bone and may have a potential as a bone augmentation material. 


\section{INTRODUCTION}

It has been demonstrated in many experimental and clinical studies in traumatology that teeth replanted after delay with a non-viable periodontal membrane will ankylose with the bone ${ }^{1-5}$. The dentin of such teeth will gradually be replaced by bone, also called replacement resorption or osseous replacement ${ }^{1-5}$. This is considered to be mainly a bone remodeling process ${ }^{1-3}$. In adults, such teeth can be left to be replaced by bone, hence preserving the height and width of the alveolar bone crest. In growing patients, such ankylosed teeth are today recommended to be decoronated and maintained in the alveolar process. The root dentin is left for replacement resorption to preserve the width and height of the alveolar bone crest and to avoid inhibition of growth of the alveolar process 6-8. In recent experimental studies, it was shown that dentin xenografts in rabbits had a potential to be incorporated in bone without inflammation and were gradually resorbed and replaced by new bone ${ }^{9,10}$.

Augmentation of bone crest deficiencies with grafting is often carried out prior to treatment with osseointegrated titanium implants ${ }^{11}$. Autogenous bone graft has been considered best because of its osteoinductive and osteoconductive properties and immunogenic compatibility ${ }^{11}$. Small autogenous bone grafts can be harvested from intra-oral sites such as lateral mandible and chin ${ }^{12}$. However, finding sufficient volumes of autogenous bone to harvest within the oral cavity may sometimes be difficult. In some situations, larger volumes of bone are required making bone grafting from extra-oral sites unavoidable. Autogenous bone can be harvested from the iliac crest but is associated with higher morbidity such as gait disturbances, pain and numbness ${ }^{13}$. Some morbidity has been reported when taking large intra-oral autogenous bone grafts from the chin region 12,14,15. Moreover, autogenous bone grafts are prone to bone resorption, which sometimes can be substantial ${ }^{11,16}$. For this reason, xenogenic bone replacement materials are today sometimes used as osteoconductive scaffolds such as hydroxyapatite, calcium triphosphate and deproteinized bovine bone either replacing the bone graft or used in combination with smaller volumes of intra-orally harvested autogenous bone ${ }^{16,17}$. Although osteoconductive, there is no osteoinductivity in these materials. For this reason, osteoinductive growth factors like bone morphogenic protein (BMP), mainly BMP-2 and BMP-7, have been in use to further promote bone healing ${ }^{18,19}$. However, the ideal carrier for BMP has not yet been found ${ }^{20}$.

Human dentin possesses osteoinductive properties possibly related to its content of bone morphogenic protein (BMP) ${ }^{21-25}$ and is therefore a carrier of BMP. However, we do not know whether dentin possesses the properties to form bone in spaces where there is no bone initially such as in the marrow space of rabbit tibia, which could be of clinical value 
for bone augmentation prior to implant placement, or whether the bone formation is mainly related only to the osteoconductive properties seen when dentin is close to bone. Our hypothesis was that dentin implanted centrally in the bone marrow will form less new bone than dentin implanted close to the native cortical bone.

Hence, the aim of this study was to further investigate whether transplanted xenogenic dentin chips inserted into the marrow space of rabbit tibia, a space where there is no solid bone initially, would contribute to bone formation and to study the pattern of such bone formation. 


\section{MATERIALS AND METHODS}

\section{Animals and anesthesia}

Ten 6-month-old New Zealand male white rabbits were used in the experiments. The experiments were carried out at the Animal Research Centre, Health Sciences Centre, Kuwait University. Thirty minutes prior to the experimental surgery, the rabbits were sedated with xylazine $\mathrm{HCl}$ (Rompun, Bayer, Leverkusen, Germany) $5 \mathrm{mg} \mathrm{kg}$ by intramuscular injection. Animals were anesthetized by intravenous injection of $35 \mathrm{mg} \mathrm{kg}^{1}$ of ketamine $\mathrm{HCl}$ (Tekan, Hikma, Amman, Jordan). The animals were kept in separate cages and fed pellets and water ad libitum throughout the duration of the study. The protocol for animal experimentation by the Animal Research Centre of the Health Sciences Center, Kuwait, was strictly adhered. Moreover, to assure a high standard, a veterinarian was administering sedation, anesthesia, and caretaking of the animals following an already used methodology ${ }^{9}, 10$.

\section{Surgical procedure}

Preparation of dentin chips for grafting

Human teeth, extracted for orthodontic reasons and then stored dry, were prepared by first removing the crowns by cutting horizontally. Then, the roots were sectioned vertically, so the pulp and root canal were exposed. The periodontal ligament and pulp were removed mechanically, and dentin chips 2-3 mm in diameter were prepared by crushing the remaining dentin in a mortar and passing it through a 2-mm grid to maximize the size of the chips. The chips were cleaned from smaller dentin particles by soaking them in $1 \%$ chlorhexidine for $5 \mathrm{~min}$ and then stored dry. The dentin chips were later inserted in the recipient tibia marrow space.

\section{Preparation of recipient sites in tibia}

The surgical areas were shaved and washed with iodine $7.5 \%$ solution, and the animals were prepared for surgery. As a supplement to general anesthesia and for vasoconstriction purposes, local anesthesia $1 \mathrm{ml}$ lidocaine hydrochloride $1 \%$ with epinephrine $5 \mathrm{lg} \mathrm{ml}{ }^{1}$ (Xylocain-adrenalin, Astra Zeneca, Södertälje, Sweden) was administered in each experimental area. Incisions were made through the skin over the superior anterior tibia bilaterally, and the bone was exposed ${ }^{9}{ }^{10}$. Bilateral tibias of ten rabbits were used in the experiment. In each tibia, a cortical bone defect was made, in which cortical bone was removed in a standardized way using a round bur and hand piece rotating 2000 rpm 9, 10. The defects were made in standardized sizes ( $6 \mathrm{~mm}$ diameter) engaging the full thickness of cortical bone until the marrow space was reached 9,10 . The bone was continuously irrigated with sterile saline during cutting to reduce thermal damage. Dentin chips were inserted through the defect into the bone marrow space until the defect was completely filled with chips. 
The incisions were closed in two layers by resorbable sutures Vicryl 4-0. Antibiotics were applied locally in the wound when suturing. Moreover, antibiotics were administered intramuscularly once daily during the first three days after surgery. The rabbits recovered in a cage with one animal per cage. The rabbits were under frequent surveillance during the postoperative period. The animals tolerated the experiments well and all survived the healing period except for one rabbit which did not survive for reasons not related to the experiment. The rabbits were sacrificed after 6 months by an overdose of ketamine. The tibia bones were dissected free from soft tissue, radiographs were taken, and histological procedures were carried out.

\section{Radiographic procedure}

Radiographs occlusal films (Kodak Insight; Eastman Kodak, Rochester, NY, USA) were taken of each tibia and were analyzed using a light viewer to localize the site in the tibia where dentin had been placed.

\section{Histological procedure}

Following surgical dissection, the samples were immersed and fixed for $48 \mathrm{~h}$ in $10 \%$ neutral buffered formalin. The samples were then decalcified in neutral EDTA for 3 months, dehydrated in alcohol and embedded in paraffin under vacuum using standard histological methods. Serial sections were cut at $5 \mathrm{Im}$ thickness and were mounted on polylysine-coated slides and then stained with hematoxylin and eosin and examined using light microscopy. The best sections, comprising both cortex and marrow areas, were selected and evaluated. Sections were evaluated with regard to tissue morphology, signs of inflammation, and formation of new bone.

\section{Quantification of bone formation}

Dentin fragments were evaluated with regard to bone formation, and the bone-dentin contact area was evaluated for each dentin fragment. The circumference area where bone was in contact with dentin was measured using a software program (Leica application suite v3.1, Leica Microsystems, Switzerland). The total circumference was then measured in micrometer around each dentin fragment. Bone formation for each dentin graft was expressed as a quotient between total bone contact area and total circumference area and expressed as a percentage. Using the same software application, the distance from each dentin fragment to the most adjacent cortical bone wall was measured.

\section{Statistical analysis}

The analysis was performed using SPSS 17.0 (SPSS, Chicago, IL, USA) for data analysis and graphical presentation. The variables, distance to closest cortical bone and percent bone-dentin fusion, were examined for normality of data with Kolmogorov-Smirnov test, 
and descriptive statistics presented as mean standard error (SE) or median with interquartile (IQ) range. The percent bone-dentin was categorized into three groups: 0, 1-50 and $>50 \%$ to find any significant difference in the mean distance for respective amount of bone formation, applying Kruskal-Wallis test. The relationship between the degree of bone formation and the distance to most adjacent cortical bone wall was ascertained with nonparametric Spearman's correlation (rho). The two-tailed probability value $P<0.05$ was considered statistically significant. 


\section{RESULTS}

Radiographs taken immediately after sacrifice verified the position of the grafted area and that in all tibias, the dentin grafts had stayed in place and were filling out the marrow spaces all the way through the bone marrow from one cortical bone wall to the to the opposite cortical bone wall at the site of grafting (Figure 1)

Figure 1. Radiograph of tibia showing the experimental area 6 months after grafting of dentin to the marrow space of tibia.

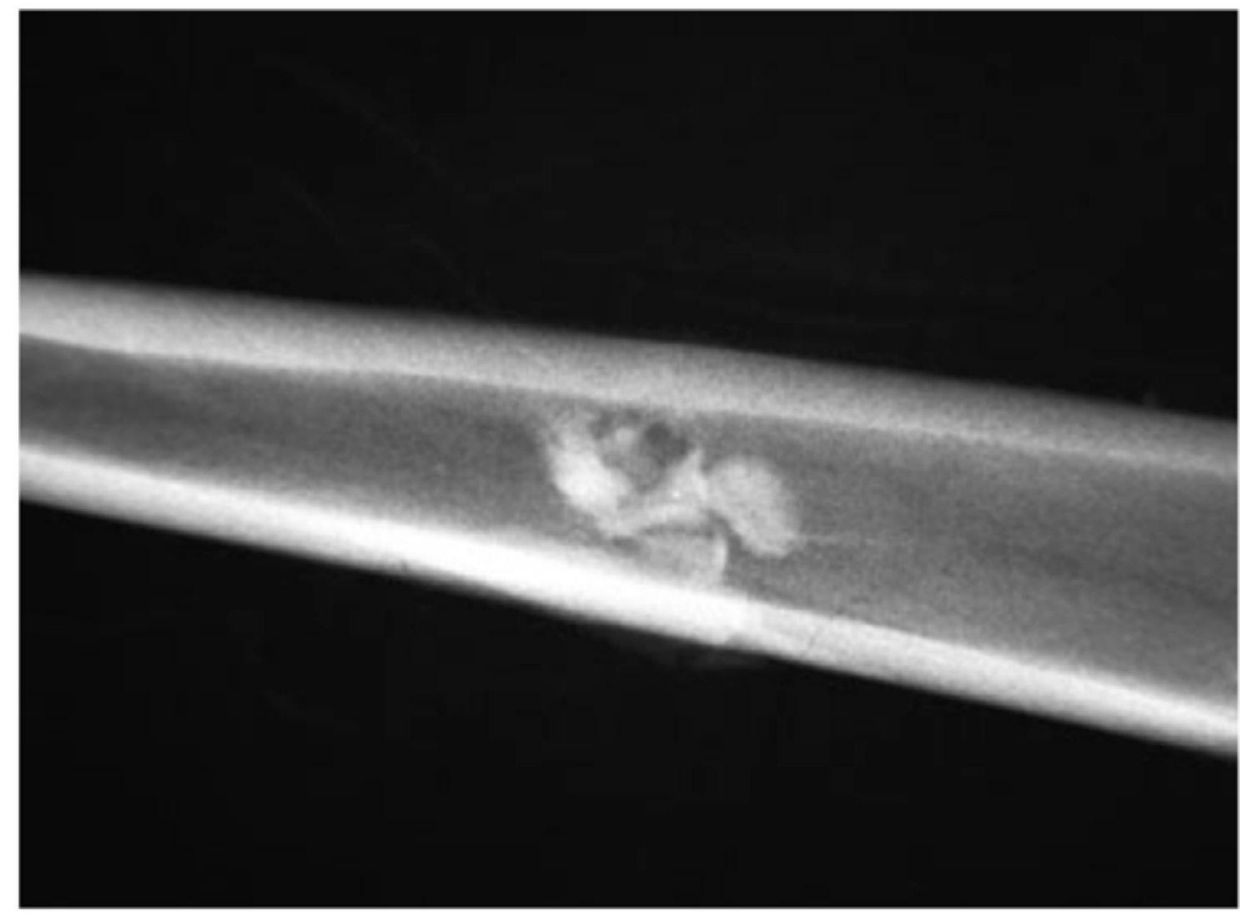

Dentin grafts can be seen in the marrow space between the cortical walls of tibia.

\section{Histological findings}

Dentin grafts were visible both in the center of the tibial marrow space as well as toward the periphery, close to the cortical bone (Figs 2 and 3). The tissue morphology was intact to clearly visualize the different cellular components, dentin grafts, and cortical bone. The inflammatory infiltrate was very minimal in the sections examined. In the areas where dentin grafts were visible in the center of the tibial marrow space, there was a lack of bone formation around the dentin grafts (Figure 2). However, fragments of calcified mate- 
rial consistent with bone were seen adjacent to dentin in certain areas with evidence of osteoclastic activity. The inflammatory component was very minimal in these areas. In areas where the dentin grafts were in close proximity to the cortical bone, new viable bone appeared to encircle the whole dentin chip (Figure 3). The newly formed bone showed both osteoblastic and osteoclastic activity.

Figure 2. Histological picture of a dentin graft centrally located in the marrow space 6 months after grafting of dentin to the marrow space.

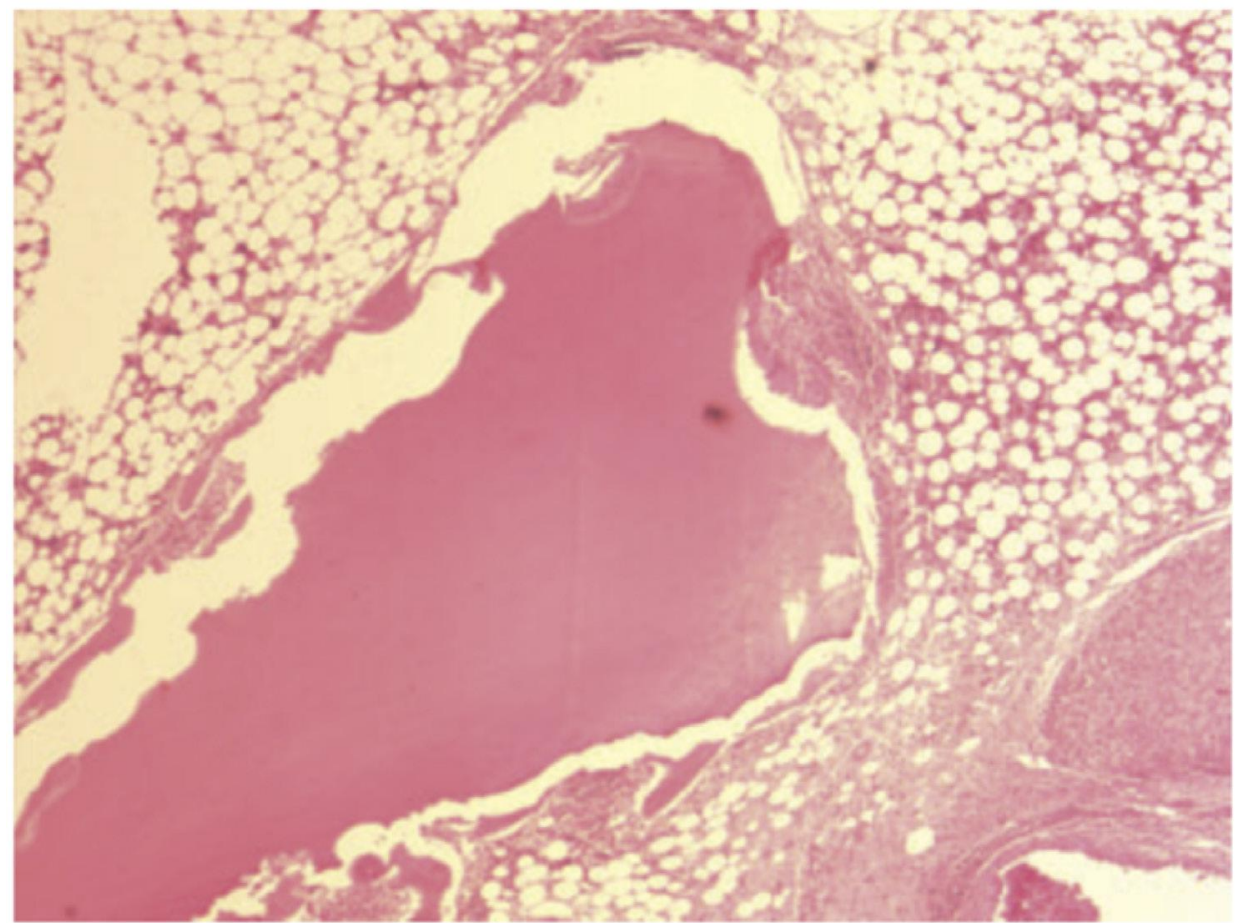

Resorption of the dentin graft can be seen. Minor or no inflammatory reaction is seen around the dentin. No new bone formation can be detected.

\section{Quantification of bone formation}

Forty-four dentin grafts in 16 tibia sections showed sufficient enough quality to be evaluated with regard to bone formation. Bone formation was ranging from 0 to $86 \%$ on the dentin fragments. Average bone formation was 22.5\% 4.36 with a median value of $5.5 \%$ (IQ 0-42\%). Bone formation was seen to a larger extent on dentin grafts located close to the tibia bone wall. Dentin grafts located centrally showed less bone formation or no bone formation at all. The distance from the dentin grafts to the nearest cortical bone wall 
ranged from $49 \mathrm{IM}$ to $2940 \mathrm{IM}(\mathrm{M}=1145, \mathrm{SE1} 1)$ and median as 1056 (IQ 387-1849). The mean distance was maximum $1630 \mathrm{IM}$ for grafts with no bone formation, while the mean distance was $614 \mathrm{IM}$ when the bone formation was more than $50 \%(P<0.001)$ (Table 1). In some of these dentin grafts, bone had been formed around the dentin graft (Figure 3). Spearman's correlation also revealed a significant negative correlation ( $r=0.579, P<$ 0.001 ) between the amount of bone formation on the dentin graft and distance to the tibia cortical wall (Figure 4).

Figure 3. Dentin graft located peripherally in the tibia marrow space 6 months after grafting.

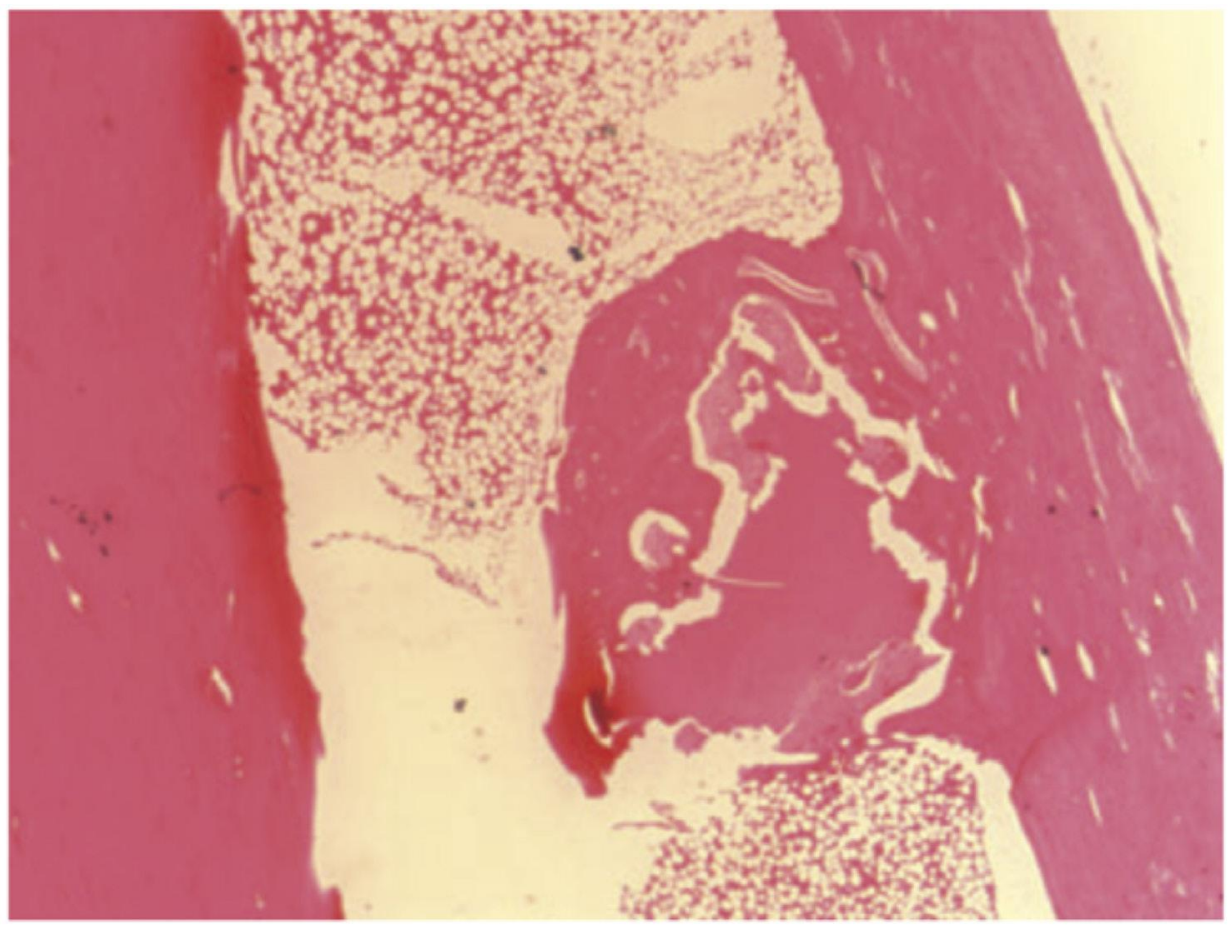

The dentin has been partially resorbed and replaced by new bone formation, which is seen surrounding the dentin graft and the new bone is in contact with the cortical wall of the tibia. 
Table 1. Bone contact around dentin chips (expressed in \%) related to distance (IM) to the nearest native cortical bone wall (expressed in mean, SE, and range)

\begin{tabular}{llll}
\hline Bone-dentin (\%) & N & Mean \pm SE & Range \\
\hline $\mathbf{0}$ & 20 & $1630 \pm 103$ & $919-2594$ \\
$\mathbf{1 - 5 0}$ & 14 & $833 \pm 239 *$ & $49-2940$ \\
$>\mathbf{5 0}$ & 10 & $614 \pm 216 *$ & $78-2356$ \\
Total & 44 & $1145 \pm 121$ & $49-2940$ \\
\hline
\end{tabular}

*0 vs $(1-50 \%)$ and $(>50 \%), P<0.001$.

Figure 4. Relation between the degree of bone formation and the distance to most adjacent cortical bone wall using nonparametric Spearman's correlation (rho), $r=0.579, P<0.001$.

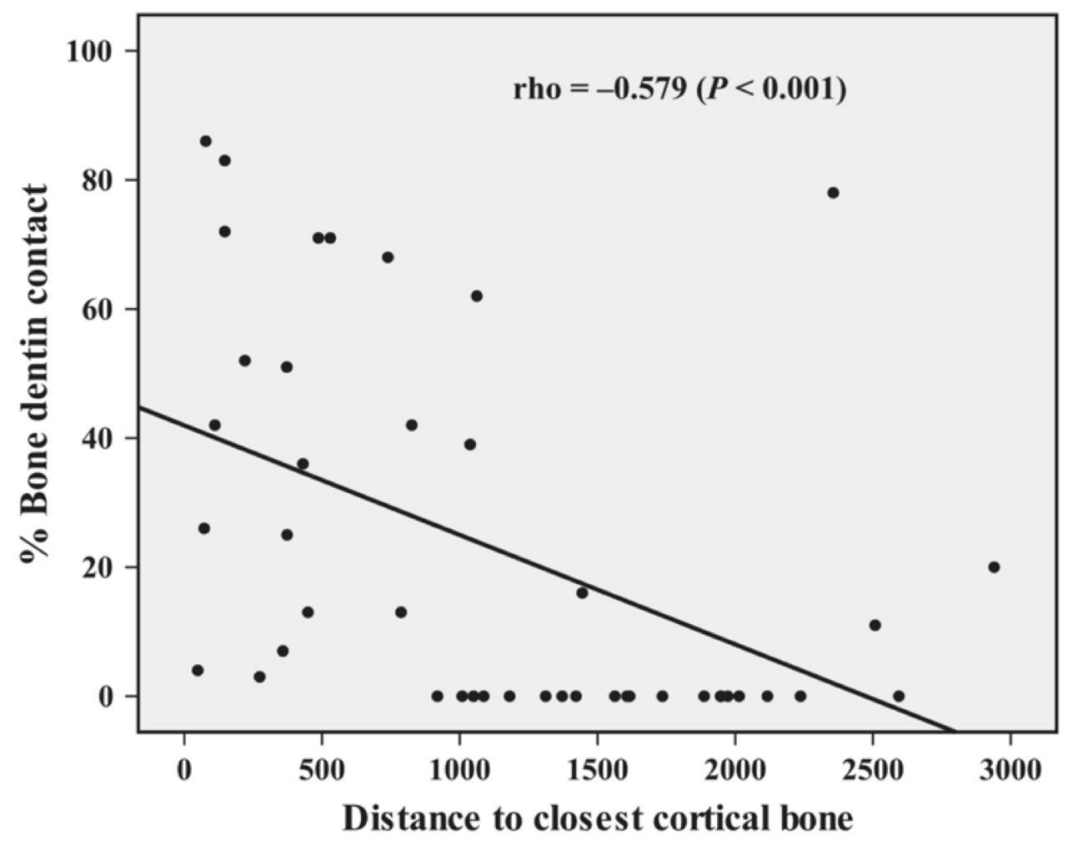




\section{DISCUSSION}

The results of the present study showed that xenogenic dentin possesses properties to form new bone when grafted into the marrow space of tibia, a space where there is no solid bone. The formation of new bone was correlated to the distance of adjacent cortical bone wall of tibia. More bone formation was seen when the dentin graft was located close to the cortical wall of tibia.

These findings may have a potential for dentin used as a bone augmentation material, for example, prior to implant placement in bone-deficient areas.

The experimental procedure was designed to study whether dentin possesses properties to form new bone when placed in a place where there is no bone initially. The tibia is a long bone consisting of a substantial marrow space where there is no bone except for the smooth native cortical walls of the tibia bone. There is an advantage in choosing this experimental model because it is easy to evaluate whether there is any bone formation inside the well-defined tibia cortex walls. Furthermore, by placing dentin grafts centrally in the marrow space, we can study properties of osteoinductivity of the dentin, whereas dentin fragments placed close to the cortex may also be subjected to osteoconductivity. Moreover, the dentin grafts are not subjected to any influence of muscular activity as if they would have been placed as onlay grafts outside the bone. Mobility due to muscular activity followed by fibrous encapsulation has been discussed in earlier studies suggested to be a factor for fibrous encapsulation when dentin was implanted in the mandibular ramus ${ }^{10}$. This may also be the reason for fibrous encapsulation of dentin grafts reported in some experiments where particulated onlay grafts on the crest underneath the periosteum in goats and dogs 26,27 . In the present study, the dentin grafts were implanted inside the tibia, hence enabling the study of bone formation on the dentin grafts protected from mobility due to muscular activity.

Radiographs were taken immediately after sacrifice with the purpose to determine the position of where the dentin chips had earlier been implanted, hence facilitating finding the correct region for the subsequent histological sectioning. However, the radiographs also gave us information that all dentin chips were seen together in the same area, and hence, no migration had taken place. Although implanted in loose marrow tissue, this tissue was apparently sufficient to keep the chips located in the same region during the experimental period.

In this study, dentin was implanted without any prior demineralization and new bone formation was found around the grafted dentin. This has earlier been reported when dentin 
has been implanted close to a muscle ${ }^{28,29}$. In those studies, both non-mineralized and mineralized dentin were used ${ }^{28,29}$. One of the studies reported that bone formation was seen 3 months later in non-demineralized dentin compared with demineralized dentin. Hence, it seems that a long time is required before new bone is formed on non-demineralized dentin grafts. In a recent study in rats, it was reported that no new bone was seen after one month in non-demineralized dentin as compared to demineralized dentin where new bone formation was seen after one month ${ }^{30}$. In our study, the experimental time was extended to 6 months, and bone formation was seen covering many of the dentin grafts.

In the present model, the new bone formation in the defect itself may also have an influence on the activity of the bone formation. This corresponds well to the clinical situation when bone or bone replacement material is used. Such materials are in clinical praxis placed adjacent to a surgical site. Furthermore, surgeons often penetrate the native cortical recipient site bone surface by making small holes in the bone in several spots to create bleeding into the grafted area to promote bone healing ${ }^{11}$. Nevertheless, more bone was formed on dentin chips and also on the cortical native bone opposite the surgical site. This finding suggests that close relation to native bone is a more important factor than being located close to the surgical defect site.

The grafts did not induce inflammatory response suggesting that immunogenicity of dentin is not an important factor. This was probably due to the fact that all soft tissues were removed from the grafted teeth before dentin particles were cut. The resorption appears to be more of a bone remodeling type similar to the replacement resorption that dentin is subjected to when replanted teeth are ankylosed ${ }^{1-5}$. This might be advantageous because in such remodeling process, dentin will be resorbed and gradually replaced by bone, hence acting as a bone replacement material. Whether replacement resorption of dentin is a solely osteoconductive or also an osteoinductive process related to the content of BMP in dentin has not been shown.

Some of the dentin chips showed more bone formation than others, and during the evaluation, we could observe a pattern in that dentin grafts located centrally in the tibia showed less new bone formation than dentin grafts located closer to the cortical bone and we decided to measure not only bone formation but also the distance to the nearest cortical wall of tibia. We found a significant negative correlation between bone formation and distance to the cortical bone wall of tibia. However, one must be aware of that in this study, we based our analysis of single sections and we cannot say anything about the area outside the section. However, in spite of this, significantly more bone was seen when chips were found more closely located to native bone in the sections examined. One reason for this may be that we are dealing mainly with osteoconductivity rather than osteoinductivity, 
namely that adjacent bone with osteoblasts is a prerequisite for bone formation so cells covering the surface of the adjacent bone more easily can reach dentin fragments located closely. However, if osteoinductivity of the dentin graft would have been a major factor, we would probably have seen more bone formed also around centrally placed dentin graft, which was not the case. There is a need for further studies aiming at assessing the role of osteoinductivity of dentin when implanted in tissue where there is no adjacent bone.

The dentin grafts located close to the cortical wall were sometimes completely surrounded by new bone (Figure 3). New bone was formed while the dentin was resorbed. This is probably a process similar to replacement resorption also seen after ankylosis of replanted teeth, which is considered a bone remodeling process ${ }^{1-5}$. It seems as if we possibly can take advantage of the replacement resorption of the dentin also when augmenting bone.

\section{CONCLUSION}

Dentin promotes new bone formation especially when located close to native cortical bone and may have a potential as a bone augmentation material.

\section{ACKNOWLEDGEMENTS}

We would like to express our gratitude to Dr. Severino Gabato for excellent sedation and taking good care of the animals and to Lovely James for excellent histological processing and assistance. We also want to thank Dr. Prem Sharma for statistical consultation. This research was funded by a research grant DS01/10 from Research Administration, Kuwait University. 


\section{REFERENCES}

1. Andersson L, Blomlöf L, Lindskog S, Feiglin B, Hammarström L. Tooth ankylosis: clinical, radiographic and histological assessments. International journal of oral surgery. 1984;13:423-31.

2. Andersson L, Bodin I, Sörensen S. Progression of root resorption following replantation of human teeth after extended extraoral storage. Dental Traumatology. 1989;5:38-47.

3. Andersson L, Lindskog S, Blomlöf L, Hedström KG, Hammarström L. Effect of masticatory stimulation on dentoalveolar ankylosis after experimental tooth replantation. Dental Traumatology. 1985;1:13-6.

4. Andreasen JO, Hjørting-Hansen E. Replantation of teeth. I. Radiographic and clinical study of 110 human teeth replanted after accidental loss. Acta Odontologica Scandinavica. 1966;24:263-86.

5. Trope M. Avulsion of permanent teeth: theory to practice. Dental Traumatology. 2011;27:281-94.

6. Andersson L, Jens O, DiAngelis AJ, David J, Kenny AS, Bourguignon C, et al. I nternational Association of Dental Traumatology guidelines for the management of traumatic dental injuries 2. Avulsion of permanent teeth. Dent Traumatol. 2012;28:88-96.

7. Andersson L, Malmgren B. The problem of dentoalveolar ankylosis and subsequent replacement resorption in the growing patient. Australian Endodontic Journal. 1999;25:57-61.

8. MALMGREN B, CVEK M, LUNDBERG M, FRYKHOLM A. Surgical treatment of ankylosed and infrapositioned reimplanted incisors in adolescents. European Journal of Oral Sciences. 1984;92:391-9.

9. Andersson L. Dentin xenografts to experimental bone defects in rabbit tibia are ankylosed and undergo osseous replacement. Dental Traumatology. 2010;26:398-402.

10. Andersson L, Ramzi A, Joseph B. Studies on dentin grafts to bone defects in rabbit tibia and mandible; development of an experimental model. Dental Traumatology. 2009;25:78-83.

11. Kahnberg K-E. Treatment of Bone-deficient Ridges in Implant Rehabilitation. Oral and Maxillofacial Surgery Chichester, West Sussex: John Wiley \& Sons. 2012:405-14.

12. Andersson $L$. Patient self-evaluation of intra-oral bone grafting treatment to the maxillary frontal region. Dental Traumatology. 2008;24:164-9.

13. Sàndor GK, Nish IA, Carmichael RP. Comparison of conventional surgery with motorized trephine in bone harvest from the anterior iliac crest. Oral Surgery, Oral Medicine, Oral Pathology, Oral Radiology and Endodontics. 2003;95:150-5

14. Nkenke E, Schultze-Mosgau S, Kloss F, Neukam F, Radespiel-Tröger M. Morbidity of harvesting of chin grafts: a prospective study. Clinical Oral Implants Research. 2001;12:495-502.

15. Raghoebar GM, Louwerse C, Kalk WW, Vissink A. Morbidity of chin bone harvesting. Clinical oral implants research. 2001;12:503-7.

16. Hallman $M$, Sennerby $L$, Lundgren S. A clinical and histologic evaluation of implant integration in the posterior maxilla after sinus floor augmentation with autogenous bone, bovine hydroxyapatite, or a 20: 80 mixture. International Journal of Oral and Maxillofacial Implants. 2002;17:635-43.

17. Lindgren C, Mordenfeld A, Hallman M. A Prospective 1-Year Clinical and Radiographic Study of Implants Placed after Maxillary Sinus Floor Augmentation with Synthetic Biphasic Calcium Phosphate or Deproteinized Bovine Bone. Clinical implant dentistry and related research. 2012;14:41-50.

18. Herford AS, Boyne PJ. Reconstruction of mandibular continuity defects with bone morphogenetic protein-2 (rhBMP-2). Journal of Oral and Maxillofacial Surgery. 2008;66:616-24.

19. Yudell RM, Block MS. Bone gap healing in the dog using recombinant human bone morphogenetic protein-2. Journal of oral and maxillofacial surgery. 2000;58:761-6.

20. Schmidmaier G, Schwabe P, Strobel C, Wildemann B. Carrier systems and application of growth factors in orthopaedics. Injury. 2008;39:S37-S43. 
Chapter 4

21. Hassan AH, Evans CA, Zaki AM, George A. Use of bone morphogenetic protein-2 and dentin matrix protein-1 to enhance the osteointegration of the Onplant system. Connective tissue research. 2003;44:30-41.

22. Ike M, Urist MR. Recycled dentin root matrix for a carrier of recombinant human bone morphogenetic protein. Journal of oral Implantology. 1998;24:124-32.

23. PINHOLTE, BANG G, HAANAES HR. Alveolar ridge augmentation by osteoinduction in rats. European Journal of Oral Sciences. 1990;98:434-41.

24. Steiglitz BM, Ayala M, Narayanan K, George A, Greenspan DS. Bone morphogenetic protein-1/Tolloid-like proteinases process dentin matrix protein-1. Journal of Biological Chemistry. 2004;279:980-6.

25. Urist M. Bone histogenesis and morphogenesis in implants of demineralized enamel and dentin. J Oral Surg. 1971;29:88-102

26. Pinholt E, Haanaes H, Donath K, Bang G. Titanium implant insertion into dog alveolar ridges augmented by allogenic material. Clinical oral implants research. 1994;5:213-9.

27. Pinholt EM, Haanaes HR, Roervik M, Donath K, Bang G. Alveolar ridge augmentation by osteoinductive materials in goats. European Journal of Oral Sciences. 1992;100:361-5.

28. Bang G, Urist MR. Bone induction in excavation chambers in matrix of decalcified dentin. Archives of Surgery. 1967;94:781-9.

29. Yeomans J, Urist M. Bone induction by decalcified dentine implanted into oral, osseous and muscle tissues. Archives of Oral Biology. 1967;12:999-IN16.

30. Mordenfeld A, Hallman M, Lindskog S. Tissue reactions to subperiosteal onlays of demineralized xenogenous dentin blocks in rats. Dental Traumatology. 2011;27:446-51. 



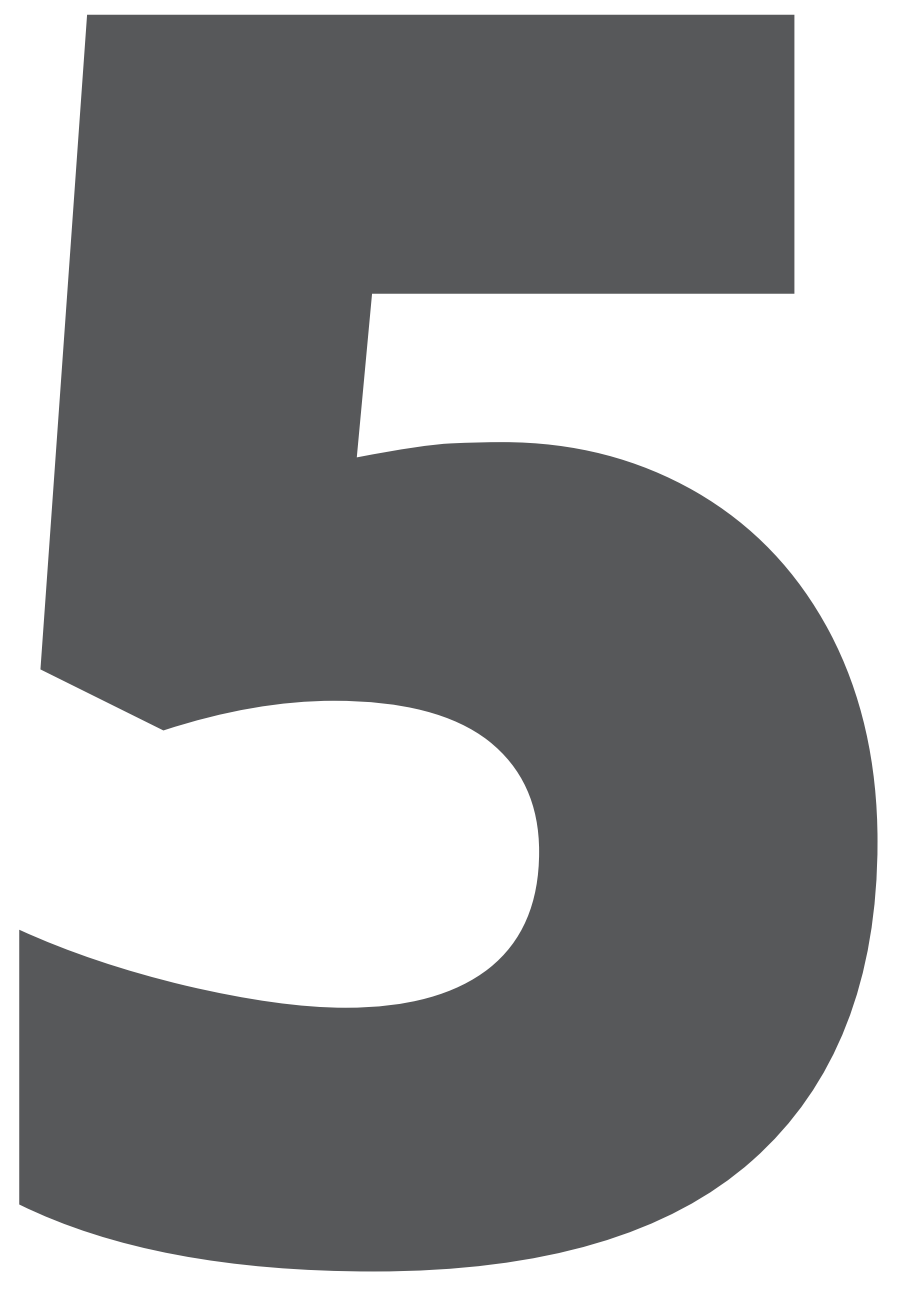




\section{"Bone Regeneration in Rabbit Calvarial Critical-Sized Defects Filled with Composite In-Situ Formed Xenogenic Dentin and Biphasic Tricalcium Phosphate/ Hydroxyapatite Mixture"}

Mohammad Kamal; Lars Andersson; Adel Al-Asfour; Alexander K. Bartella; Felix Gremse; Stefanie Rosenhain; Severino Gabato; Frank Hölzle; Peter Kessler; Bernd Lethaus

Published in the Journal of Biomedical Materials Research Part B: Applied Biomaterials. 2018. doi: 10.1002/jbm.b.34171 


\section{ABSTRACT}

Objectives: The purpose of this study was to evaluate bone healing in calvarial defects using two bone graft substitute materials; biphasic beta-tricalcium phosphate/hydroxyapatite in hydrogel (ß-TCP/HA) versus composite non-demineralized xenogenic dentin with B-TCP/HA mixture.

Material and Methods: Full thickness critical-sized defects were created bilaterally in 10 New Zealand male rabbits. Seven defects were left empty, six filled with biphasic tricalcium phosphate putty, and seven were filled with composite non-demineralized xenogenic dentin with biphasic tricalcium phosphate. Animals were sacrificed at eight weeks postoperatively and the healing of the biomaterial-filled defects was compared radiographically and by histomorphometry. Micro-computed tomography $(\mu \mathrm{CT})$ was utilized to analyze the osteogenesis and healing patterns of the defects. Quantitative analysis of volume fraction (\%) of the newly formed bone and remaining graft material (FV=filling volume/TV=tissue volume) and mean intensity [HU] in the defects were evaluated.

Results: Defects filled with composite dentin with biphasic tri-calcium phosphate showed volume fraction (FV/TV) in the order of $55.81 \% \pm 17.72 \%$, whereas defects filled with only biphasic tricalcium phosphate showed a fraction of $39.84 \% \pm 16.06 \%$, which represent the ratio of remaining graft material and new bone formation to the tissue volume. The empty negative control defects showed a volume fraction of $19.14 \% \pm 8.787 \%$. Histological analysis showed significant percentage increase in bone formation and residual graft with the composite Dentin/ß-TCP group after 8 weeks.

Conclusions: The findings suggest that composite xenogenic dentin with biphasic tricalcium phosphate showed improved osteogenesis when compared to biphasic tricalcium phosphate without the addition of non-demineralized dentin. 


\section{INTRODUCTION:}

Bone grafting is a surgical procedure widely utilized in craniofacial surgery to fill skeletal voids and reconstruct bone defects, and to promote biological wound healing. Autogenous bone graft is the current gold standard, but several factors have limited its use such as the need for a second surgery for harvesting of the bone graft with donor bone supply limitation, surgical donor site morbidity, reported inherent resorption of the bone graft and unpredictable graft volume stability, and increased surgical operation time and costs to patients. ${ }^{1-7}$

Lately, large number of biodegradable bone replacement materials have been developed as a biological alternative to conventional bone grafting given the recent technological advances in biomedical science to overcome these issues. Several resorbable and non-resorbable bone substitute materials have been utilized as suitable replacement materials for craniofacial reconstruction including processed allografts 8,9 , xenografts ${ }^{10}$, and alloplasts such as bioactive ceramics like hydroxyapatite and tricalcium phosphate 2, 8, 11, 12, and polymer-based materials like polyether ether ketone and fiber-reinforced bioglass. 3,12

Calcium phosphate ceramics have largely been used in clinical settings as bone replacement materials given their biocompatibility and osteoconductive properties, and have been shown to promote new bone formation in various interventional studies in human and translational animal models. ${ }^{2,8,9,11-14}$ Various forms of calcium phosphate bone substitute materials exist, however, calcium hydroxyapatite (HA) and beta-tricalcium phosphate ( $\beta$-TCP) are commonly utilized in biphasic form as bone graft substitute. Both materials are proportionally combined to obtain a suitable balance between the predictable biodegradability offered by $\beta$-TCP, and the resiliency with improved mechanical properties offered by HA. 2, 14

Dentin, a natural organic component of teeth, has also been investigated as an effective bone grafting substitute material. It has been shown in several human and in-vivo models that dentin promotes new bone regeneration with inherent osteoinductive and osteoconductive properties. 10,15-22 Several forms of allogenic and xenogenic dentin forms have been investigated as a potential bone replacement material including processed bovine dentin, allogenic freeze-dried dentin, demineralized dentin, and dried particulate dentin (chips and blocks). ${ }^{22}$

Interventional in-vitro and in-vivo testing of novel bone substitute materials requires reliable biological models to evaluate bone healing and compare clinical effectiveness of 
osteogenesis. In-vivo calvarial critical-size defects animal model is a robust and reliable model to test for the healing capacity and osteogenesis pattern of novel bone replacement materials. 3, 9, 12, 23 The embryonic origin of the rabbit calvarium develops from a membranous precursor mimicking the intramembranous ossification of the facial bones, making the calvarial critical-sized defects a suitable model for translational studies in craniofacial research. ${ }^{8,24,25}$

Our aim in this study was to assess the impact of composite in-situ formed non-demineralized xenogenic dentin particles and biphasic calcium phosphate putty carrier (60\% HA /40\% ß-TCP ratio; Maxresorb inject, Botiss Biomaterials, Berlin, Germany) on graft resorption, bone healing, and osteogenesis in a rabbit calvarial model. 


\title{
MATERIALS AND METHODS:
}

\author{
Animals, Anesthesia, and Housing
}

The procedure was conducted at the Animal Research Centre, Health Sciences Centre, Kuwait University. All animals were handled in compliance with European Communities Council Directive of 24 November 1986 (86/609/EEC) for the care of laboratory animals in experimental procedures and ethical guidelines for animal research. ${ }^{26}$ All methods were carried out in accordance with the approved guidelines and regulations of the Kuwait Health Sciences Center Ethical Committee for the use of Laboratory Animals in Teaching and in Research, Kuwait University.

Ten 8-week-old male New Zealand White rabbits weighing 2.7 to 3.0 kilograms were used for the study. The rabbits were sedated thirty minutes prior to the experimental surgery with Xylazine HCl 5 mg/kg (Rompun, Bayer, Leverkusen, Germany) by intramuscular injection and subsequently anesthetized by intravenous injection of $35 \mathrm{mg} / \mathrm{kg}$ of ketamine $\mathrm{HCl}$ (Tekan, Hikma, Amman, Jordan). To ensure a high standard of animal care, a veterinarian was administering the sedation, anesthesia, and care-taking of the animals following an already used methodology. ${ }^{15,19}$ The animals were kept in separate cages and fed pellets and water throughout the duration of the study. The rabbits were cared for accordingly per protocol and observed by veterinarian until the end of the study.

\section{Preparation of grafting materials}

Xenogenic dentin was prepared from human teeth that were extracted for orthodontic reasons. Teeth were prepared by first by drying then sectioning the crowns and roots in a vertical way to expose the root canal. The remaining periodontal ligament and pulp material were mechanically removed. Dentin was further processed into finer chips 2-3 $\mathrm{mm}$ in diameter by crushing them in a mortar and a 2-mm grid. The processed dentin was cleansed from dentin debris by soaking them in 1\% chlorhexidine for 5 min and then stored dry. For the control grafting intervention and formation of the composite graft, a commercially available biphasic ß-TCP/HA injectable putty bone-substitute material made of $60 \%$ HA/40\% ß-TCP ratio was utilized and prepared per manufacturer's protocol (Maxresorb inject, Botiss Biomaterials, Berlin, Germany). ß-TCP/HA putty and dentin chips were then mixed together intraoperatively by the same operator to ensure consistency of preparation and volume proportion, and to create a moldable grafting mixture. The mixture was made using 0.5 cc of Maxresorb inject (60\% hydroxyapatite and $40 \% \beta$-tricalcium phosphate, Botiss Biomaterials, Berlin, Germany) with predetermined volume of dentin blocks obtained using the same dental scoop excavator (Figure 1). 
Figure 1: Calvarial defects surgery and insertion of testing materials.
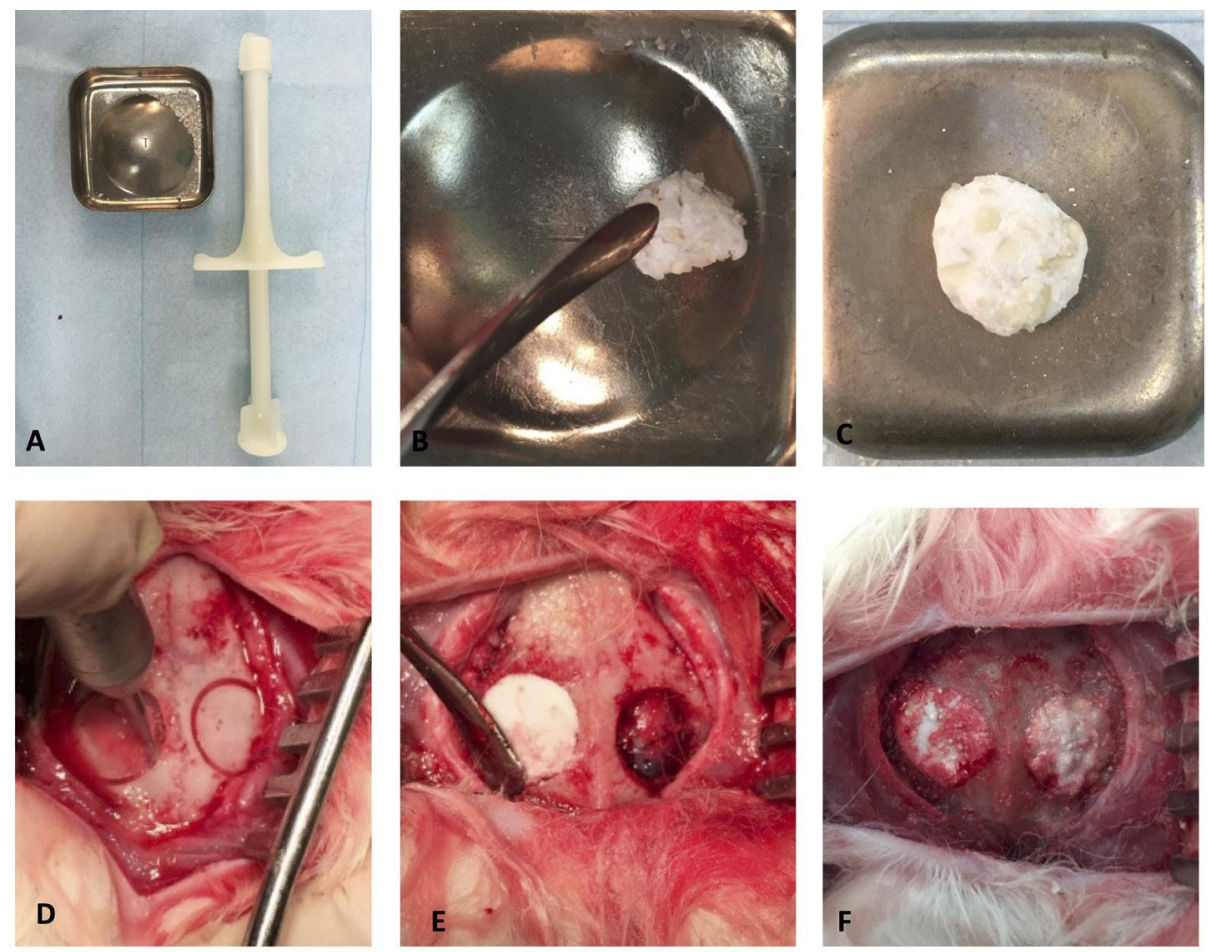

(A) Non-demineralized xenogenic human dentin chips and injectable B-TCP Mixture (Maxresorb Inject). (B) In-situ mixing phase. (C) Formed composite materials. (D) Creation of calvarial defects. (E) Insertion of grafting intervention. (F) Two calvarial defects filled with two different intervention prior to wound closure.

\section{Preparation of surgical sites in Calvarium}

A vertical incision $4 \mathrm{~cm}$ in length was performed down to the periosteum using scalpel along the midsagittal suture. The periosteum was then incised vertically using the scalpel and carefully pushed to the lateral direction using periosteal elevator to expose the calvarial bone. $13 \mathrm{~mm}$ full thickness critical-sized defects were created bilaterally in the calvarium of NZW rabbits using $1.7 \mathrm{~mm}$ Steiger carbide bur with round end (Stryker Instruments, Kalamazoo, Michigan, USA) and a surgical handpiece drill operating at 1,500 r.p.m under copious irrigation with physiological saline to avoid thermal and traumatic injury to the underlying dura. 7 defects were left empty as negative control, 6 filled with $\beta$-TCP putty (Maxresorb inject, Botiss Biomaterials, Berlin, Germany), and 7 were filled with composite in-situ formed non-demineralized xenogenic dentin mixed with biphasic $\beta$-TCP (Figure 1). Multi-layers wound closure was performed by carefully suturing the galea aponeurotica then the skin using resorbable sutures (Vicryl®, Ethicon Inc., New Jersey, USA). 


\section{Animal sacrifice and qualitative evaluation}

Animals were sacrificed at 8 weeks after the operation with an intravenous lethal dose of T61 Euthanasia Solution (Embutramide, Mebezonium iodide, Tetracaine hydro-chloride; Hoechst GmbH, Frankfurt, Germany) after sedation with an intramuscular injection of Xylazine $\mathrm{HCl} 5 \mathrm{mg} / \mathrm{kg}$. Block sections of the calvarial specimens were obtained using an oscillating saw maintaining the adjacent galea and soft tissue and fixed in 10\% neutral-buffered formalin. The specimens were grossly inspected for inflammation. Specimens were subsequently scanned with micro-computed tomography $(\mu \mathrm{CT})$ before histological processing. Healing of the biomaterial-filled defects was compared radiographically and by histomorphometry. $\mathrm{HCT}$ was utilized to analyze the graft resorption, osteogenesis, and healing patterns of the defects with different interventions. Quantitative analysis filling volume fraction (\%) of the remaining graft material and new bone formation (FV=filling volume/TV=tissue volume) and its mean radio-density in the defects filled with each group was evaluated.

\section{Acquisition of $\mu C T$ scans, segmentation, and statistical analysis}

After animal sacrifice, the calvarial shelf with defects filled with various materials were harvested for ex vivo $\mu \mathrm{CT}$ imaging. To acquire the $\mu \mathrm{CT}$ images, skulls were imaged ex vivo in HCT (Tomoscope 30s Duo, CT-Imaging, Erlangen, Germany) according to a protocol previously described. ${ }^{27}$ After positioning in a multimodal bed, skulls were scanned with the $\mu C T$, a dual energy scan (HQD-6565-360-90) which acquires 720 projections with $1032 \times 1012$ pixels with scanning time $90 \mathrm{sec}$ per subscan was used. To cover one skull two subscans were acquired. Both tubes of the dual source $\mu \mathrm{CT}$ were operated with voltage of $65 \mathrm{kV}$ and current of $1 \mathrm{~mA}$. The $\mu \mathrm{CT}$ data was reconstructed at an isotropic voxel size of $35 \mu \mathrm{m}$ using a Feldkamp type algorithm and a smooth kernel and the Hounsfield units were calibrated with a water phantom. For analysis, the $\mu C T$ data was down-sampled using binning to a voxel size of $70 \mu \mathrm{m}$. To determine the filling volume fraction and mean density the $70 \mu \mathrm{m} \mu \mathrm{CT}$ file was analyzed with the Imalytics Preclinical Software (Gremse-IT GmbH, Aachen, Germany) (Figure 2). ${ }^{28}$ Two cylindrical stencils (diameter $10 \mathrm{~mm}$, height $5 \mathrm{~mm}$ ) were used to segment both defect areas (Figure 3). Each cylinder was created separately to avoid overlapping at the inside. A threshold operation below -500 HU was applied to remove air and a threshold operation over $600 \mathrm{HU}$ was performed to segment the bones (remaining from surgery and newly formed) and the filling material inside the cylinder (Figure 4). Relative volume (filling material and bone /tissue volume) and mean radio-density of the bone and bone graft material (as HU-calibrated density score) inside the defect area were determined. The statistical data was analyzed using GraphPad Prism (Version 7.0 for Windows, GraphPad Software, La Jolla, California, USA). To compare the measurements between TCP, composite and control, one-way ANOVAs were performed in combination with Tukey post-tests. A p-value below 0.05 was considered to represent statistical significance. 
Figure 2: Bone regeneration in both defect areas was evaluated using $\mu \mathrm{CT}$ imaging taken at 8 weeks after intervention (3D images).
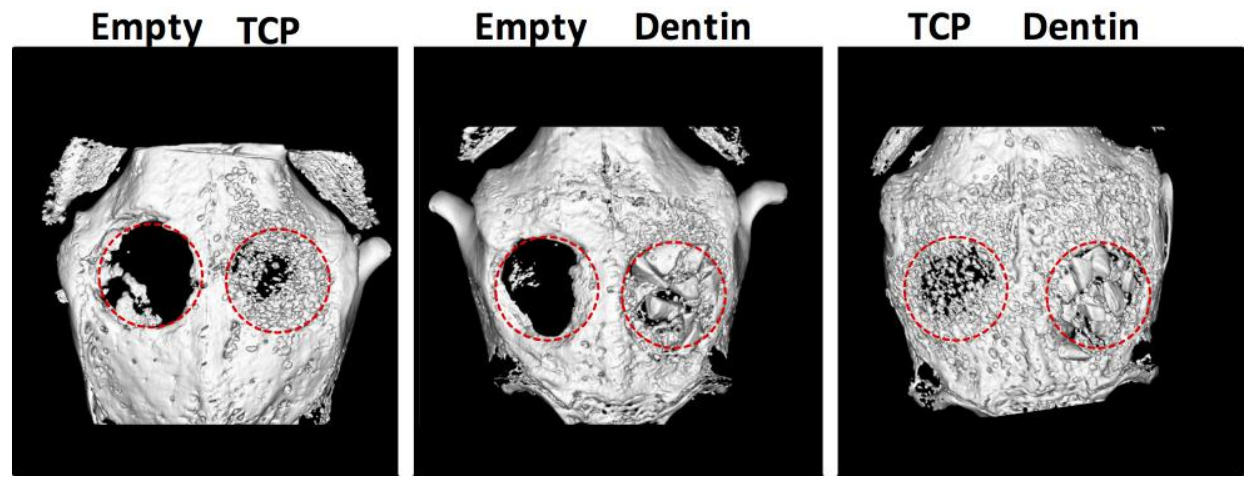

Figure 3: Segmentation algorithm to determine bone volume and bone brightness via two cylinder-stencils.

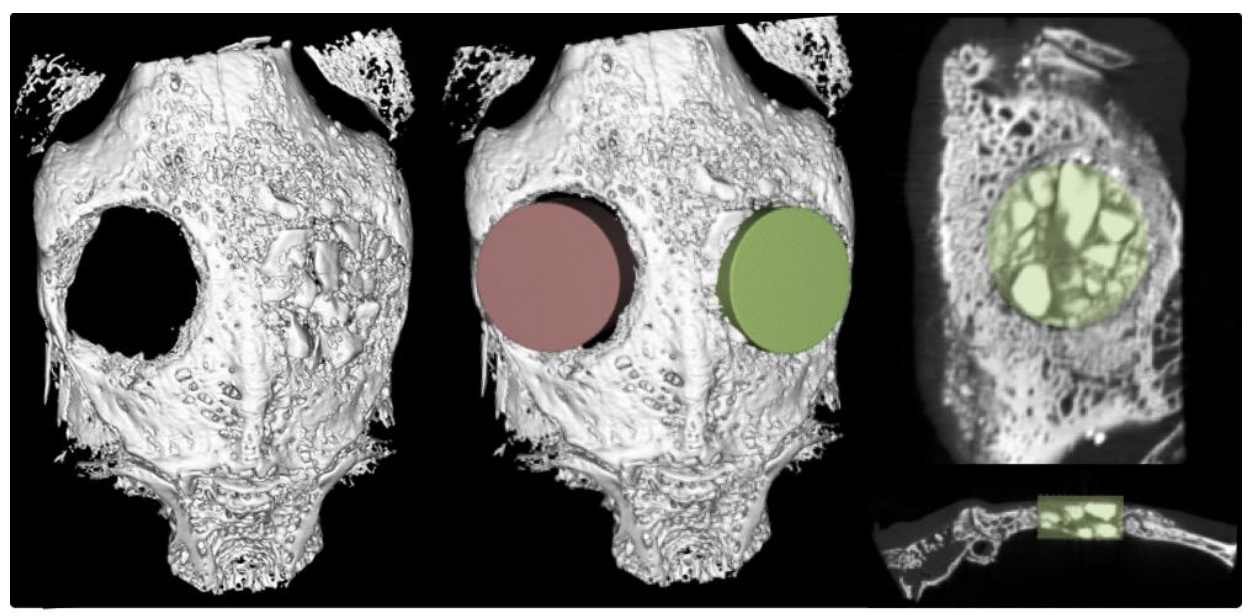

The red cylinders analyze the left defect area, here it is empty representing the control, and the green cylinder measures the right defect area, here dentin. Right images depict coronal and sagittal $\mu C T$ slices.

Histologic preparation histomorphometry, and statistical analysis

After $\mu \mathrm{CT}$ image acquisition, the specimens were dehydrated using ascending ethanol gradient (50-100\%) prior to embedding in methylmethacrylate resin (Technovit 9100, Heraeus Kulzer $\mathrm{GmbH}$, Germany). Coronal sections of the embedded undecalcified specimens were obtained at a thickness of about $200 \mu \mathrm{m}$ using the EXAKT cutting unit (EXAKT Technologies Inc., Oklahoma City, Oklahoma, USA), then thinned and polished manually to a final thickness of about 50-70 $\mu \mathrm{m}$. Final specimens were stained with Masson's Trichrome (MTC) 
and Toluidine blue (TB) according to protocol, and were analyzed using light microscopy (Leitz, Wetzlar, Germany) at a magnification of 1.25. Histomorphometric evaluation for performed with Image software (ImageJ 1.51p, National Institute of Health, Bethesda, Maryland, USA) by manually measuring the compartments of new bone area, and remaining grafting materials. Mean percentage of bone formation per total defect volume $(\%=$ new bone area / total defect area) and standard deviation (SD) were used for each group. Statistical analysis conducted using GraphPad Prism and one-way ANOVA was performed in combination with a Tukey post-test. A p-value below 0.05 was considered to represent statistical significance. 


\section{RESULTS:}

\section{Micro-computed tomography imaging}

The results from $\mu \mathrm{CT}$ showed that the empty defects expressed only partial bone filling with bone, mostly from the margin of the defects (Figure 4). This indicates that after 8 weeks of healing, ossification did not complete in these defects. However, the two intervention groups with B-TCP and composite B-TCP /Dentin showed bridging of bone and filling of the defects at the 8-week time. Statistical analysis of the radiographic parameters showed that there is significance difference in the Hounsfield unit (HU) between the empty negative control defects $(241.9 \pm 171)$ and B-TCP filled defects $(858.3 \pm 441)$ versus the composite (Dentin/ß-TCP) filled defects (1609 \pm 552.1), which had the significant highest values (Figure 4, Table 1). Likewise, when comparing the percentage filling volume Fraction (FV/TV), which represents the percentage of bone (remaining cortical bone and newly formed bone) and bone graft material in relation to the tissue, there was a statistically significant difference across all groups, with the significant highest percentage of bone and bone graft material in the composite Dentin/ß-TCP filled defects $(55.81 \% \pm 17.72)$ when compared to the ß-TCP filled defects (39.84 \pm 16.06$)$ and the empty, negative control defects (19.14 \pm 8.787) (Figure 4, Table 1). Additional findings were that Dentin was found outside of the right defect area in one single case, whereas ß-TCP materials outside of the right defect was present in most skulls (Figure 5).

Table 1. Quantification of the $\mu \mathrm{CT}$ measurements:

\begin{tabular}{lccc}
\hline & N & Filling Fraction (FV/TV) [\%] & Mean Density [HU] \\
\hline Control & 7 & $19.14 \pm 8.787$ & $241.9 \pm 171$ \\
B-TCP & 6 & $39.84 \pm 16.06$ & $858.3 \pm 441$ \\
Composite (Dentin/B-TCP) & 7 & $55.81 \pm 17.72$ & $1609 \pm 552.1$ \\
\hline
\end{tabular}

Filling volume fraction (FV/TV), and mean radio-density (HU) in control and defect groups, (means \pm standard deviations).

\section{Histomorphometric analysis}

Histological analysis after 8 weeks showed bone healing pattern which correlated well with the radiographic findings (Figure 6, Table 2). Both ß-TCP and Dentin/ß-TCP filled cavities showed new bone formation (NBA/TDA). Histomorphometric analysis showed higher percentage $(p<0.05)$ of bone formation volume in Dentin/ß-TCP group $(59,61 \% \pm 7,3)$ compared to ß-TCP group $(36,65 \% \pm 6,16)$, and the empty filling group $(8,43 \% \pm 2,15)$. Present of residual grafts was significantly higher $(p<0.0001)$ in Dentin/ß-TCP groups with grossly visible dentin chips (Figure 6, Table 2). On higher magnification, dentin was fused with bone (ankylosis) with the presence of resorption cavities mostly within the ß-TCP material (Figure 7). 
Figure 4: Filling ratio of bone and graft material and density in both defect areas was evaluated using $\mu \mathrm{CT}$ imaging.
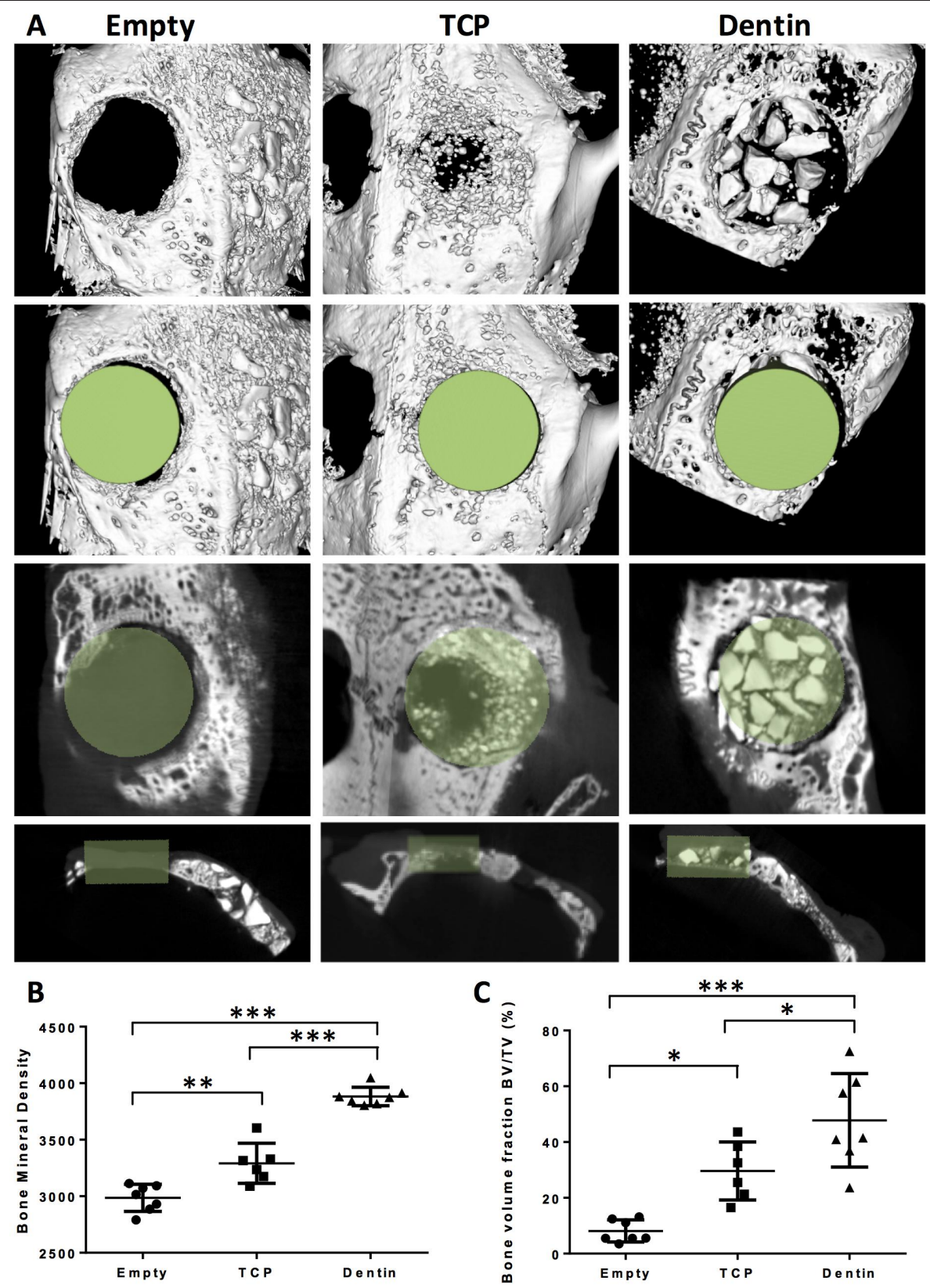

(A) $\mu C T$ images of bone regeneration taken at 8 weeks after intervention (top to bottom: 3D image, segmentation with cylinder stencil in the defect area, coronal plane, and axial plane). (B) Quantitative analysis of filling volume fraction (FV= volume of graft material and bone / TV=tissue volume) in the defect areas of each group. (C) Radio-density (in Hounsfield Units) of the tissue inside the defect region including remaining and newly formed bones, filling material, and excluding air. Significant differences are indicated by, ${ }^{*} p<0.05,{ }^{*} p<0.01$, ${ }^{* * *} p<$ 0.0001). 
Figure 5: $\mu \mathrm{CT}$ images 8 weeks after intervention of dislocated bone growth on the calvarial surface outside the defect areas, different perspectives.
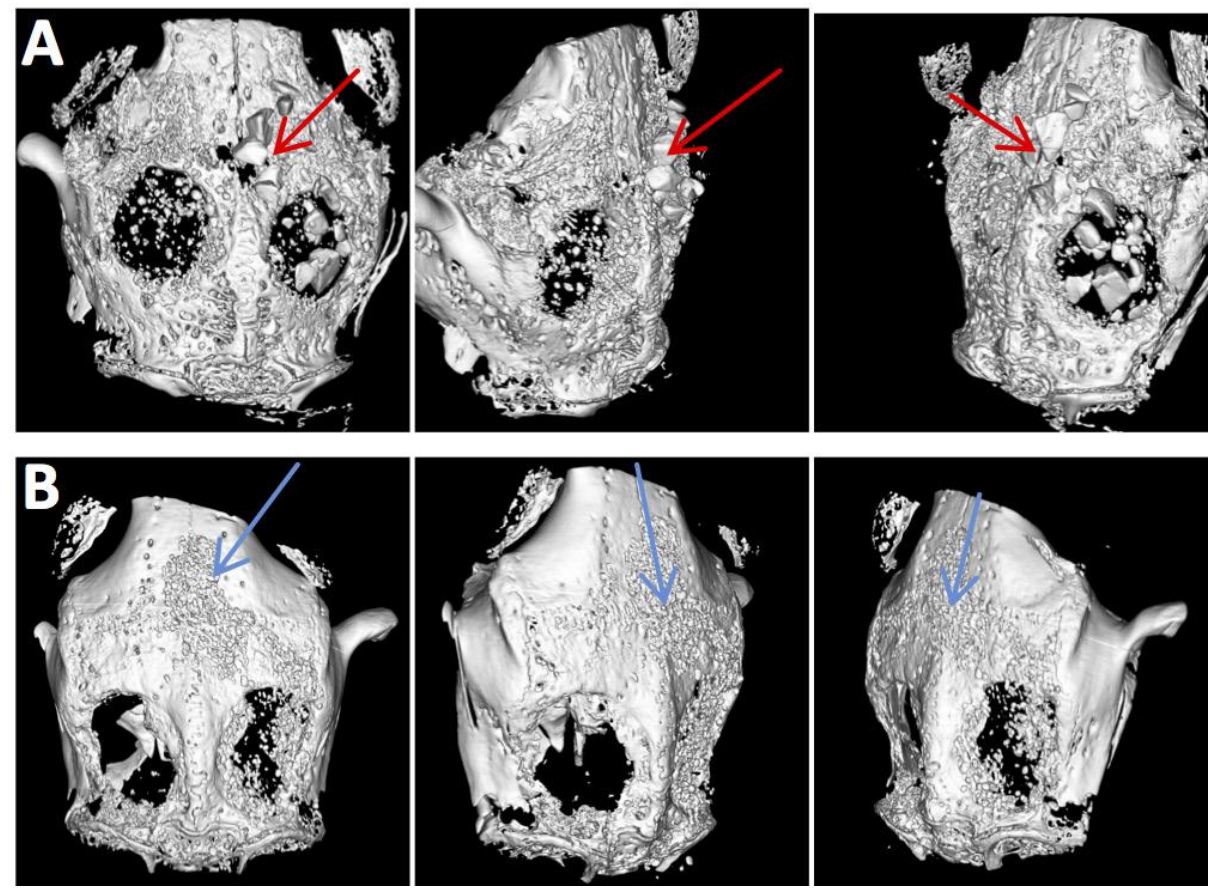

(A) Dentin outside of the right defect area was found only in one single case (red arrows). (B) TCP outside of the right defect area was present in most skulls (blue arrows).

Table 2. Results of histomorphometry measurements

\begin{tabular}{lccc}
\hline & N & \% Bone Formation & \% Residual Graft \\
\hline Control & 7 & $8,43 \pm 2,15$ & - \\
B-TCP & 6 & $36,65 \pm 6,16$ & $18,37 \pm 4,09$ \\
Composite (Dentin/B-TCP) & 7 & $59,61 \pm 7,3$ & $48,11 \pm 8,11$ \\
\hline
\end{tabular}

(NBA: new bone area, TDA: total defect area, RGA: residual graft area). \% Bone Formation=NBA/TDA×100 and \% Residual Graft=RGA/TDA in defect groups, (means \pm standard deviations) 
Figure 6:

A

Masson's Trichrome
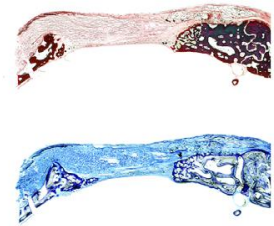

Toluidine blue

ß-TCP
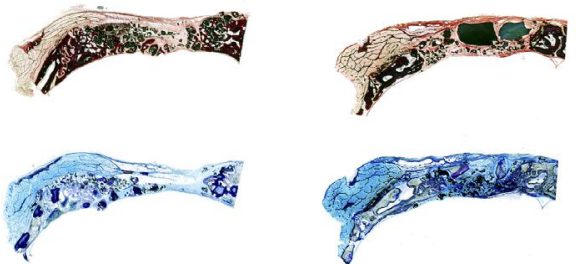

B
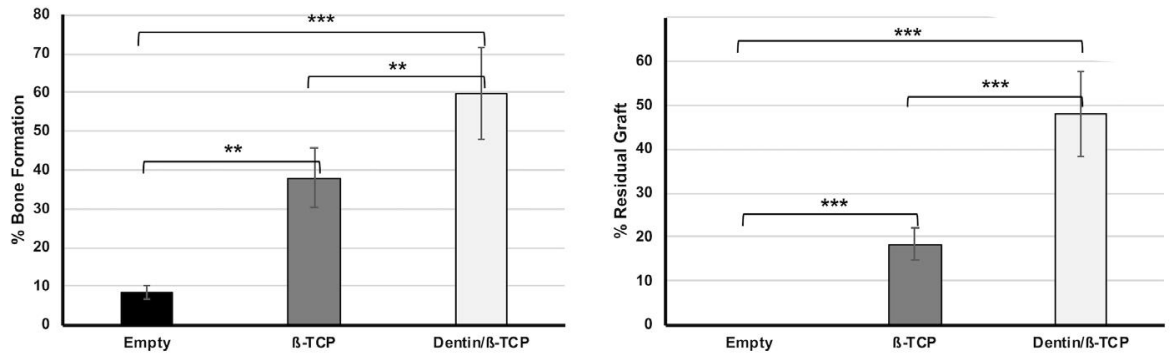

(A) Histological representation of the filled defects from each group. (B) Histomorphometric analysis representing percentage bone formation and percentage residual grafts in the defect area after 8 weeks (\% Bone Formation= new bone area / total defect area $\times 100 ; \%$ Residual Graft= residual graft area / total defect area). Significant difference between groups as indicated: $* P<0.05, * * P<0.001, * * * P<0.0001$ (ANOVA with post hoc test).

Figure 7: Histological representation of the filled calvarial defects. Histological sections of a healed calvarial defect 8 weeks after insertion of the grafting intervention using composite dentin/B-TCP stained with Toluidine Blue (A) and Masson's Trichrome (B).

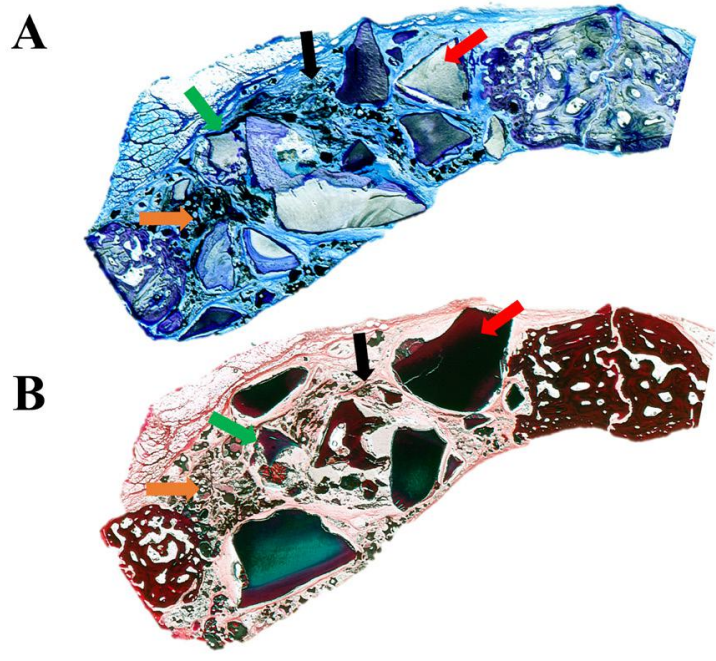

Red arrow: residual dentin; black arrow: newly formed bone; green arrow: fused dentin with bone; orange arrow: resorption lacunae associated with ß-TCP. Original magnification $\times 10$. 


\section{DISCUSSION:}

In our present comparative study of bone healing and osteogenesis in calvarial defects in rabbits, the $\mu \mathrm{CT}$ and histomorphometric analysis results showed improved graft stability and higher radio-density after eight weeks in the composite Dentin/ ß-TCP group, indicating that this hybrid biomaterials have higher osteoconductive properties compared to the ß-TCP alone.

Numerous bone graft substitutes are available in clinical practice including xenogenic bone and alloplastic grafts. There is a heterogeneous group of synthetic biomaterial substitutes, most common is calcium sulfate, tricalcium phosphate and the recently introduced beta phase TCP (B-TCP). ${ }^{29,30}$ Bone substitute materials based on B-TCP have been further developed in powder granules, blocks, and as injectable paste. A mixture of ß-TCP granules in a water-based gel with nano-micro HA granules has been developed to create a non-hardening and a moldable putty-like paste, like Maxresorb (Maxresorb inject, Botiss Biomaterials, Berlin, Germany). Since they also lack osteoindutive properties, alloplastic materials are often subject to prolonged healing time. Moreover, preservation of the volume of the graft is dependent on the level of resorbability of the bone substitute materials and replacement by bone. ${ }^{30-32}$

To alter the resorption properties of $\beta$-TCP bone substitutes, the material can be synthesized by changing the mixing ratio of $\mathrm{HA}$ and $\beta-\mathrm{TCP}$, and many studies reported a HA/ $\beta-\mathrm{TCP}$ ratio between 65:35 and 55:45 to be clinically appropriate and allows for a balanced combination of resorption rate with simultaneous ingrowth of bone formation. ${ }^{29,33-35}$

The HA is less susceptible to resorption and acts as structural scaffold for ingrowing osteogenesis and angiogenesis while the dissolution of $\beta$-TCP during grafting procedures yields $\mathrm{Ca}$ and $\mathrm{PO} 4$ ions, which have also been suggested to stimulate bone formation. ${ }^{29,30,36}$

Several previous experimental and interventional studies have demonstrated that delayed replantation of teeth with non-viable periodontal ligaments will fuse with bone in a process called ankylosis. ${ }^{15,37-41}$ The ankylosed teeth, which are mostly made of dentin, will gradually resorb and subsequently undergo osseous replacement in a process called replacement resorption, and it is mostly attributed to bone remodeling process. 15, 17-19, 37, 39, 40 Several studies have focused on this biological phenomenon to preserve alveolar ridge height and width by delayed replantation of teeth or decoronation of the tooth for retention in the alveolar process to be slowly replaced by bone, thus maintaining the volume of alveolar process and preventing resorption of the bone. ${ }^{38,40,42,43}$ 
Recent experimental studies in rabbits showed that xenogenic dentin block grafts were healed and integrated into native bone with minimal inflammatory reaction, and subsequently were slowly remodeled and replaced by new bone. ${ }^{18,19}$ Fusion of bone with dentin was seen in $86 \%$ of the dentin surface after 3 months and $98 \%$ after 6 months. Resorption of the dentin and remodeling was also seen after 6 months with osseous replacement in the resorption cavities, however, the resorption process was gradual with subsequent replace of dentin with new bone. The lack of inflammation during healing noted in several studies can be suggestive of the low level of immunogenicity with dentin, and it's not a determining factor in graft healing. ${ }^{15,18,19}$

In addition, it has been shown in previous studies that human dentin carries osteoinductive properties, likely to the inherent reservoir of bone morphogenic protein (BMP) and functions as a carrier of these growth factors. ${ }^{15,44-46}$

Another study by Al-Asfour et. al. evaluated the osteoinductivity of dentin, their results showed that xenogenic non-demineralized dentin also has the potential to form new bone when inserted in the marrow space of rabbits tibia. ${ }^{15}$ Their results also showed that more bone was found on the graft when the graft was placed close to native bone, suggesting osteoconductive properties are more important than osteoinductive properties. ${ }^{15}$ It was also shown in another study by the same research group that when dentin was implanted in non-osteogenic environment very little bone was formed suggesting that ostoinductivity is low in non-demineralized dentin. ${ }^{17}$

Mordenfeld et. al. evaluated the effect of demineralization of xenogenic porcine dentin on bone formation in an osteoconductive environment in a rat model. ${ }^{47}$ Their study demonstrated higher rate of resorption of the dentin grafts with increasing level of pre-grafting demineralization of dentin. ${ }^{47}$ On interest was their finding that increasing the degree of demineralization also resulted in a significant increase in bone formation, given that inflammatory reaction around the dentin graft was minimal. This suggests that inflammatory reaction during grafting with dentin plays a crucial role during healing and bone regeneration process and dentin can be incorporated in bone when inflammation is absent, so that it can gradually resorb and be replaced with bone. ${ }^{15,18,19,32,47}$ Additional finding reported by Mordenfeld et. al. was that when dentin blocks were loosely implanted under the periosteum of rat skull, fibrous encapsulation compromising graft uptake was seen as a result of the micromobility during the osteogenic phase of bone healing. However, when dentin blocks were placed in a tighter pocket limiting their mobility of the graft, better graft incorporation was seen. ${ }^{47}$ This is consistent with other studies demonstrating that dentin blocks undergo resorption without inducing bone formation when implanted in animal non-osteogenic environment, such as in the muscle or the subcutaneous tissue, 
due to mobility of the graft and leading to fibrous tissue formation. ${ }^{15,17,48,49}$ Therefore, local grafting environment, stability of the graft, and susceptibility to inflammatory reactions seems to play a crucial role with regard to incorporation of the dentin grafts during healing phase. ${ }^{15,17-19,38,47,48}$ Additional study by Al-Asfour et. al. compared only bone regeneration pattern in rabbit's tibia between autogenous bone blocks and xenogenic demineralized dentin blocks. Their results demonstrated similar resorption rate and characteristics of the xenogenic demineralized dentin blocks to the autogenous bone over 12 weeks period, with notable new bone formation through replacement of the integrated dentin at the native bone/dentin graft interphase. ${ }^{16}$ By forming a mixture of dentin blocks and a ß-TCP/ HA putty, we aimed at improving the surgical handling of the dentin blocks and allowing more stability of the composite in the defect to promote the osteogenic potential associated with dentin.

Our results show that calvarial defects filled with composite Dentin/ß-TCP showed the highest radio-density (Hounsfield Units:1609 \pm 552.1) and percentage bone and graft volume (FV/TV) at $55.81 \pm 17.72$ (Figure 4, Table 1). This indicates that both Dentin and ß-TCP/HA mixture successfully healed with the native bone. It should be noted that our HU values should be higher than values measured with clinical devices, because our high resolution preclinical scanner operates at lower $x$-ray voltages which leads to higher calcium-based signal intensities in HU compared to clinical CT scanners. The higher \% filling volume fraction can be likely attributed to the dentin grafts, which show a slower rate of resorption and may possess inherent osteoinductive properties. This correlated well with previous finding reported by Al-Asfour, Andersson, and Mordenfeld groups. ${ }^{15-19,47}$ Our histological findings correlated well with our radiographic findings, suggesting enhanced bone formation in the composite groups in addition to higher percentage of residual graft in the defects (Figure 6, Table 2). Noteworthy was the presence of a higher percentage residual graft in the Dentin/ß-TCP group (48.11 \pm 8.11$)$ compared to the ß-TCP group (18,37 \pm 4,09 ) after a healing period of 8 weeks duration (Table 2). This is consistent with previous studies which have demonstrated that dentin exhibits a slower resorption rate during graft healing and leading to enhanced maintenance of the graft volume. 15, 16, 18, 19, 32, 47, 48 In our study, the ß-TCP/HA mixture was non-hardening, and served the purpose of stabilized the dentinal granules to allow osteogenesis and expression of any osteoinductive properties. 


\section{CONCLUSION:}

Our results show superior graft stability, bone healing and osteogenesis in calvarial critical-sized defects in rabbits filled with composite Dentin/B-TCP over B-TCP and control groups. The results suggest that adding non-demineralized dentin to $B$-TCP improves healing properties and promotes bone new bone formation. This may be of importance in the clinic when using bone replacement materials.

\section{ACKNOWLEDGMENTS:}

The authors are grateful to Professor George Sandor from the department of Oral and Maxillofacial Surgery, Oulu University Hospital, Oulu, Finland for sharing his experience on calvarial surgery procedures in rabbits. 


\section{REFERENCES}

1. Nyström $E$, Ahlqvist J, Legrell PE, Kahnberg K-E. Bone graft remodelling and implant success rate in the treatment of the severely resorbed maxilla: a 5-year longitudinal study. International journal of oral and maxillofacial surgery. 2002;31:158-64.

2. Saikia K, Bhattacharya T, Bhuyan S, Talukdar D, Saikia S, Jitesh P. Calcium phosphate ceramics as bone graft substitutes in filling bone tumor defects. Indian journal of orthopaedics. 2008;42:169.

3. Lappalainen O-P, Karhula S, Haapea M, Kyllönen L, Haimi S, Miettinen S, et al. Bone healing in rabbit calvarial critical-sized defects filled with stem cells and growth factors combined with granular or solid scaffolds. Child's Nervous System. 2016;32:681-8.

4. Nyström E, Ahlqvist J, Kahnberg K-E, Rosenquist J. Autogenous onlay bone grafts fixed with screw implants for the treatment of severely resorbed maxillae: Radiographic evaluation of preoperative bone dimensions, postoperative bone loss, and changes in soft-tissue profile. International journal of oral and maxillofacial surgery. 1996;25:351-9.

5. Nyström E, Ahlqvist J, Gunne J, Kahnberg K-E. 10-year follow-up of onlay bone grafts and implants in severely resorbed maxillae. International journal of oral and maxillofacial surgery. 2004;33:258-62.

6. Gordh MA, Per. Some basic factors essential to autogeneic nonvascularized onlay bone grafting to the craniofacial skeleton. Scandinavian journal of plastic and reconstructive surgery and hand surgery. 1999;33:129-46.

7. Gordh M, Alberius P, Johnell O, Lindberg L, Linde A. Osteopromotive membranes enhance onlay integration and maintenance in the adult rat skull. International journal of oral and maxillofacial surgery. 1998;27:67-73.

8. Clokie CM, Moghadam H, Jackson MT, Sandor GK. Closure of critical sized defects with allogenic and alloplastic bone substitutes. Journal of Craniofacial Surgery. 2002;13:111-21.

9. Haddad AJ, Peel SA, Clokie CM, Sándor GK. Closure of rabbit calvarial critical-sized defects using protective composite allogeneic and alloplastic bone substitutes. Journal of Craniofacial Surgery. 2006;17:926-34.

10. Su-Gwan K, Hak-Kyun K, Sung-Chul L. Combined implantation of particulate dentine, plaster of Paris, and a bone xenograft (Bio-Oss ${ }^{\circledR}$ ) for bone regeneration in rats. Journal of Cranio-Maxillofacial Surgery. 2001;29:282-8.

11. Zhou AJ-J, Peel SA, Clokie CM. An evaluation of hydroxyapatite and biphasic calcium phosphate in combination with Pluronic F127 and BMP on bone repair. Journal of Craniofacial Surgery. 2007;18:1264-75.

12. Lappalainen O-P, Karhula SS, Haapea M, Kauppinen S, Finnilä M, Saarakkala S, et al. Micro-CT Analysis of Bone Healing in Rabbit Calvarial Critical-Sized Defects with Solid Bioactive Glass, Tricalcium Phosphate Granules or Autogenous Bone. Journal of oral \& maxillofacial research. 2016;7.

13. Janssen NG, de Ruiter AP, van Hout WM, van Miegem V, Gawlitta D, Groot FB-d, et al. Microstructured $\beta$-Tricalcium Phosphate Putty Versus Autologous Bone for Repair of Alveolar Clefts in a Goat Model. The Cleft Palate-Craniofacial Journal. 2016.

14. Bittermann GKP, Janssen NG, van Leeuwen M, van Es RJ. One-Year Volume Stability of Human Facial Defects Filled With a $\beta$-Tricalcium Phosphate-Hydroxyl Apatite Mixture (Atlantik). Journal of Craniofacial Surgery. 2014;25:372-4.

15. Al-Asfour A, Andersson L, Kamal M, Joseph B. New bone formation around xenogenic dentin grafts to rabbit tibia marrow. Dent Traumatol. 2013;29:455-60.

16. Al-Asfour A, Farzad P, Al-Musawi A, Dahlin C, Andersson L. Demineralized Xenogenic Dentin and Autogenous Bone as Onlay Grafts to Rabbit Tibia. Implant dentistry. 2017;26:232-7.

17. Al-Asfour A, Farzad P, Andersson L, Joseph B, Dahlin C. Host tissue reactions of non-demineralized autogenic and xenogenic dentin blocks implanted in a non-osteogenic environment. An experimental study in rabbits. Dent Traumatol. 2014;30:198-203 
18. Andersson L. Dentin xenografts to experimental bone defects in rabbit tibia are ankylosed and undergo osseous replacement. Dent Traumatol. 2010;26:398-402.

19. Andersson L, Ramzi A, Joseph B. Studies on dentin grafts to bone defects in rabbit tibia and mandible; development of an experimental model. Dent Traumatol. 2009;25:78-83.

20. Fugazzotto P, De Paoli S, Benfenati S. The use of allogenic freeze-dried dentin in the repair of periodontal osseous defects in humans. Quintessence international (Berlin, Germany: 1985). 1986;17:461.

21. Gomes MF, dos Anjos MJ, Nogueira TO, Guimaraes SA. Histologic evaluation of the osteoinductive property of autogenous demineralized dentin matrix on surgical bone defects in rabbit skulls using human amniotic membrane for guided bone regeneration. The International journal of oral \& maxillofacial implants. 2001;16:563-71.

22. Moharamzadeh K, Freeman C, Blackwood K. Processed bovine dentine as a bone substitute. British Journal of Oral and Maxillofacial Surgery. 2008;46:110-3.

23. Humber CC, Sándor G, Davis JM, Peel SA, Brkovic BM, Kim YD, et al. Bone healing with an in situ-formed bioresorbable polyethylene glycol hydrogel membrane in rabbit calvarial defects. Oral Surgery, Oral Medicine, Oral Pathology, Oral Radiology, and Endodontology. 2010;109:372-84.

24. Hussain I, Moharamzadeh K, Brook IM, José de Oliveira Neto P, Salata LA. Evaluation of osteoconductive and osteogenic potential of a dentin-based bone substitute using a calvarial defect model. International journal of dentistry. 2012;2012.

25. Schmitz JP, Hollinger $\mathrm{JO}$. The critical size defect as an experimental model for craniomandibulofacial nonunions. Clinical orthopaedics and related research. 1986;205:299-308.

26. Directive C. 86/609/EEC of 24 November 1986 on the approximation of laws, regulations and administrative provisions of the Member States regarding the protection of animals used for experimental and other scientific purposes. Off J Eur Commun. 1986;29:L358.

27. Gremse F, Doleschel D, Zafarnia S, Babler A, Jahnen-Dechent W, Lammers T, et al. Hybrid HCT-FMT imaging and image analysis. Journal of visualized experiments: JoVE. 2015.

28. Gremse F, Stärk M, Ehling J, Menzel JR, Lammers T, Kiessling F. Imalytics Preclinical: interactive analysis of biomedical volume data. Theranostics. 2016;6:328.

29. Daculsi G. Biphasic calcium phosphate concept applied to artificial bone, implant coating and injectable bone substitute. Biomaterials. 1998;19:1473-8.

30. Lindgren C, Sennerby L, Mordenfeld A, Hallman M. Clinical histology of microimplants placed in two different biomaterials. International Journal of Oral and Maxillofacial Implants. 2009;24:1093-100.

31. Hallman $\mathrm{M}$, Thor $\mathrm{A}$. Bone substitutes and growth factors as an alternative/complement to autogenous bone for grafting in implant dentistry. Periodontology 2000. 2008;47:172-92.

32. Mordenfeld A, Hallman M, Johansson CB, Albrektsson T. Histological and histomorphometrical analyses of biopsies harvested 11 years after maxillary sinus floor augmentation with deproteinized bovine and autogenous bone. Clinical Oral Implants Research. 2010;21:961-70.

33. Ducheyne P, Radin S, King L. The effect of calcium phosphate ceramic composition and structure on in vitro behavior. I. Dissolution. Journal of Biomedical Materials Research Part A. 1993;27:25-34.

34. Gauthier O, Bouler J-M, Aguado E, Legeros R, Pilet P, Daculsi G. Elaboration conditions influence physicochemical properties and in vivo bioactivity of macroporous biphasic calcium phosphate ceramics. Journal of Materials Science: Materials in Medicine. 1999;10:199-204.

35. Schwartz $C$, Liss $P$, Jacquemaire $B$, Lecestre $P$, Frayssinet $P$. Biphasic synthetic bone substitute use in orthopaedic and trauma surgery: clinical, radiological and histological results. Journal of Materials Science: Materials in Medicine. 1999;10:821-5.

36. Jensen SS, Broggini N, Hjørting-Hansen E, Schenk R, Buser D. Bone healing and graft resorption of autograft, anorganic bovine bone and $\beta$-tricalcium phosphate. A histologic and histomorphometric study in the mandibles of minipigs. Clinical oral implants research. 2006;17:237-43. 
37. Andersson L. Dentoalveolar ankylosis and associated root resorption in replanted teeth. Experimental and clinical studies in monkeys and man. Swedish dental journal Supplement. 1988;56:1-75.

38. Maslamani M, Almusawi A, Joseph B, Gabato S, Andersson L. An experimental model for studies on delayed tooth replantation and ankylosis in rabbits. Dental Traumatology. 2016;32:443-9.

39. Andersson L, Blomlöf L, Lindskog S, Feiglin B, Hammarström L. Tooth ankylosis: clinical, radiographic and histological assessments. International journal of oral surgery. 1984;13:423-31.

40. Andersson L, Bodin I, Sörensen S. Progression of root resorption following replantation of human teeth after extended extraoral storage. Dental Traumatology. 1989;5:38-47.

41. Trope M. Avulsion of permanent teeth: theory to practice. Dental Traumatology. 2011;27:281-94.

42. Malmgren B. Ridge preservation/decoronation. Journal of endodontics. 2013;39:S67-S72.

43. Malmgren B, Tsilingaridis G, Malmgren O. Long-term follow up of 103 ankylosed permanent incisors surgically treated with decoronation-a retrospective cohort study. Dental Traumatology. 2015;31:184-9.

44. Ike M, Urist MR. Recycled dentin root matrix for a carrier of recombinant human bone morphogenetic protein. Journal of Oral Implantology. 1998;24:124-32.

45. Steiglitz BM, Ayala M, Narayanan K, George A, Greenspan DS. Bone morphogenetic protein-1/Tolloid-like proteinases process dentin matrix protein-1. Journal of Biological Chemistry. 2004;279:980-6.

46. Urist M. Bone histogenesis and morphogenesis in implants of demineralized enamel and dentin. Journal of oral surgery (American Dental Association: 1965). 1971;29:88.

47. Mordenfeld A, Hallman M, Lindskog S. Tissue reactions to subperiosteal onlays of demineralized xenogenous dentin blocks in rats. Dental Traumatology. 2011;27:446-51.

48. Machado M, Souza A, Araujo V. Histological evaluation of the osteoinduction capability of human dentine. International endodontic journal. 2006;39:855-9.

49. Morris ML. The implantation of decalcified human dentin and cementum into the subcutaneous tissues of the rat. Journal of periodontal research. 1967;2:273-81. 



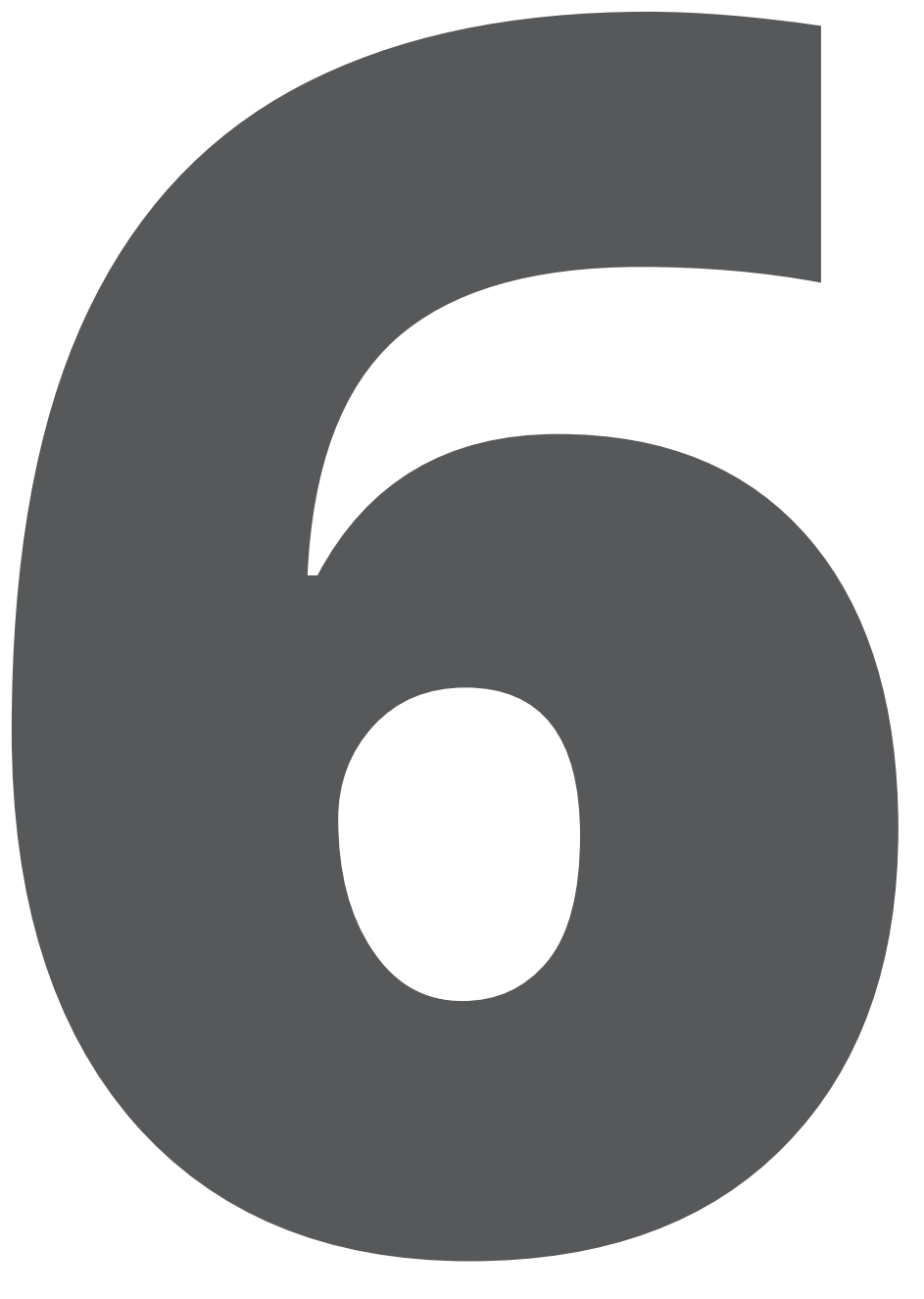




\section{"A Rabbit Model for Experimental Alveolar Cleft Grafting"}

Mohammad Kamal; Lars Andersson; Rene Tolba; Alexander Bartella; Felix Gremse; Frank Hölzle; Peter Kessler; Bernd Lethaus

Published in the Journal of Translational Medicine. 2017 Feb 24;15(1):50. doi: 10.1186/s12967-017-1155-2 


\section{ABSTRACT}

Objectives: The purpose of the present study was to develop an animal model for creating alveolar cleft defects with properly simulated clinical defect environment for tissue-engineered bone-substitute materials testing without compromising the health of the animal. Cleft creation surgery was aimed at creating a complete alveolar cleft with a wide bone defect with an epithelial lining (oral mucosa) overlying the cleft defect.

Methods: A postmortem skull of a New Zealand White (NZW) rabbit skull (Oryctolagus cuniculus) underwent an osteological and imaging survey. A pilot postmortem surgery was conducted to confirm the feasability of a surgical procedure and the defect was also radiologically confirmed and illustrated with micro-computed tomography. Then, a surgical in vivo model was tested and evaluated in sixteen $(n=16) 8$-week-old New Zealand White (NZW) rabbits to create in-vivo alveolar cleft creation surgery.

Results: Clinical examination and imaging analysis 8 weeks after cleft creation surgery revealed the establishment of a wide skeletal defect extending to the nasal mucosa simulating alveolar clefts in all of the rabbits.

Conclusions: Our surgical technique was successful in creating a sizable and predictable model for bone grafting material testing. The model allows for simulating the cleft site environment and can be used to evaluate various bone grafting materials in regard to efficacy of osteogenesis and healing potenital without compromising the health of the animal. 


\section{BACKGROUND}

Congenital alveolar cleft is a malformation occurring as a result of non-fusion of primary palate during weeks 4 to 12 of gestation. The goal of alveolar cleft repair is to establish bony continuity of the alveolar ridge in the maxilla, seal the communication oro-nasal communication, and create a favorable anatomy for dental rehabilitation. ${ }^{1-3}$ Reconstruction of the these defects is done via the alveolar cleft bone grafting procedure using autologous bone, allogenic and xenogeneic bone grafting materials, along with various tissue-engineered bone replacement materials. ${ }^{48}$

Optimising the quality of the existing bone grafting materials and looking for novel and better bone-substitute materials is crucial in improving the clinical outcome. Experimental testing of various grafting materials requires the pre-establishment of a proper biological model to conduct experimental studies and evaluate the clinical effect with respect to osteogenesis and healing. Animal models with simulated alveolar clefts are considered appropriate as an experimental model for testing of clinical interventions. Several animal models have been utlized for testing of alveolar cleft grafting materials including mice, rats, rabbits, cats, dogs, swines, goats, sheep and monkeys. ${ }^{4,9-24}$

Development of alveolar clefts in experimental animals can be achieved, either surgically created or congenitally induced in utero during embryonic development. 19, 25-27 Previous studies on in utero congenitally induced models reported increased need of technical expertise, concurrent multiple fetal malformations, and an increased incidence of intrauterine fetal death and abortions. In addition, several studies reported that newborn animal models with lip defects were less cared by their mothers and some being subjected to cannibalism. ${ }^{28-30}$ Next to congenitally induced models, surgically created alveolar clefts in animals also seem suitable to experimental studies regarding histologic and biomechanical properties of bone grafting material.

Moreover, it is essential to allow proper timing for healing of the defect and establish an alveolar cleft of appropriate width mimicking the human scenario of a skeletal defect extending to the nasal mucosa and the adjacent teeth and be suitable for clinical testing. Some earlier reported cleft models do not correspond to the clinical situation since a bone defect is created and filled in the same session. This is not in accordance to the real situation in which the defect is covered by epithelial lining. Hence, it is important to achive a bony cleft with its surfaces covered with healthy mucosal tissue at the time of placement of graft. For this reason it is necessary to first create the bone defect and then in a second stage surgery, after healing with mucosal lining of the cleft has been achieved, place the grafting material, otherwise the defect is not corresponding to the real clinical 
situation. Moreover, compared with congenitally induced alveolar clefts, surgically created cleft models in animals can easily be created and allow for controlling the size and extent of the bony cleft and properly position the overlying soft tissue to serve the purpose of the model.

Animals used in biomaterial bone research include small animals, such as mice, rats, guinea pigs, and rabbits, and large animal category mostly goats, dogs, and primates. 31-33 Rodent models have inherent limitations when compared to larger models, including rabbits. Rodents have smaller long-bones, more fragile cortex, and do not show Haversian-type remodeling in the cortex. ${ }^{31}$ Rabbits are considered the largest animals in the small animals category, and hence less susceptible to elaborate and exhaustive additional clearance requirements usually implemented by the central ethical committees. They are non-aggressive, easy to observe, have quicker vital capacity in terms of gestation and maturity, and can be locally bread. ${ }^{31-33}$ The histology of bone in rabbits is not quite similar to bone in humans, and composes of dense Haversian bone and layers primarily vascular longitudinal canals. ${ }^{31-33}$ However, similarities in bone mineral density and fracture toughness between rabbits and human have been reported in the literature. ${ }^{32-34}$ An essential issue with rabbits that they express rapid skeletal metabolism and increased bone turnover rate, mostly cortical remodeling when compared to primates and some rodents. 31-33 To properly simulate human in-vivo environment, the rabbit model is an appropriate animal model for alveolar cleft experimental studies because the rabbit is reproducible, accurate, easy to house and handle, relatively easily anaesthetised, provides large enough area for testing and properly sized mammalian that can bear the trauma of surgery. ${ }^{32,33}$

The aim of this study was to develop a model in New Zealand White (NZW) rabbits, Oryctolagus cuniculus, to enable surgically creating of a healed skeletal alveolar defect extending to the nasal mucosa and the adjacent tooth structure as seen in human patients. 


\section{METHODS}

\section{Osteological survey of New Zealand White rabbit skull (Oryctolagus cuniculus):}

A Skull of a NZW rabbit was obtained from the animal stock library at RWTH Aachen University Hospital (Germany) for inspection of the anatomical landmarks. (Figure 1)

\section{Microfocus Computed Tomography (Micro-CT) for imaging survey:}

Imaging with Micro-CT of a rabbit skull were obtained of radiographic analysis of the skeletal anatomy for assessment of the feasibility of creating an alveolar cleft defect and planning of the cleft creation surgery. Images were evaluated using cross-sectional slices and rendered three-dimentional reconstruction. (Figure 2)

Figure 1: Osteological survey of New Zealand White rabbit skull.
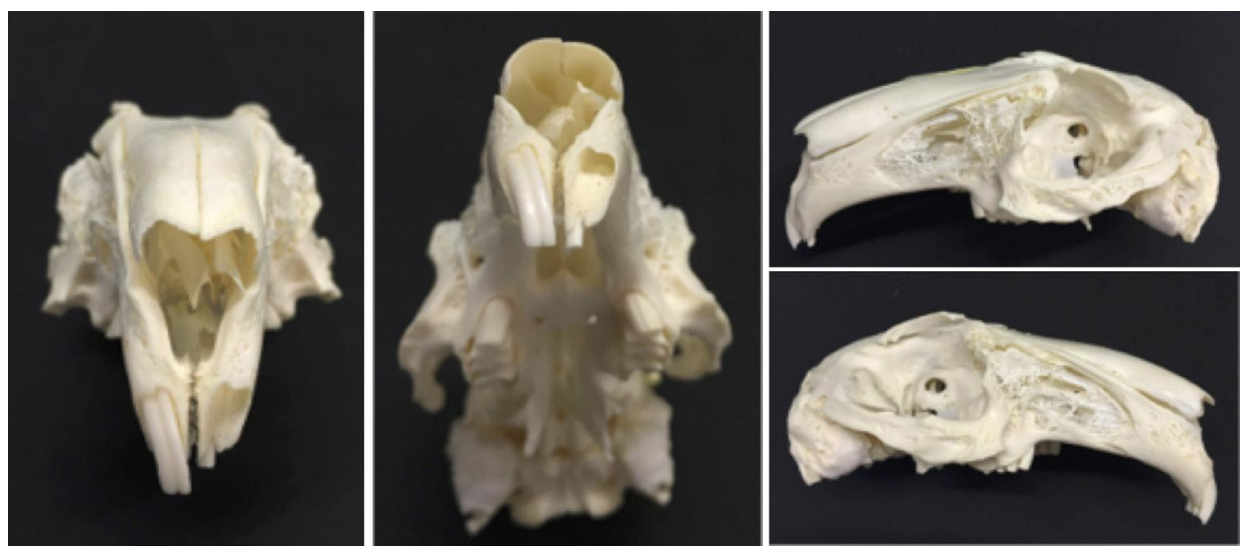

Preserved skull of a NZW rabbit (Oryctolagus cuniculus) showing the depth and orientation of the maxillary incisor alveolus after removing the central incisor. The alveolus penetrates into the maxillary bone laterally with strong curvature toward the palatal shelf. The tooth extends just shorts of the extensively aerated maxillary sinus.

\section{Postmortem pilot alveolar cleft creation surgery and Micro-CT imaging:}

A pilot surgical operation of the planned procedure was conducted on a sacrificed rabbit head. There was no need for an animal ethical committee approveal given that the procedure was performed on a sacrificed animal which was previously been used in another animal testing project. The cleft creation surgery was conducted according to the proposed procedure. (Figure 3) Post-operative Microfocus Computed Tomography (Micro-CT) was obtained of the rabbit skull to evaluate the created postmortem defect. (Figure 4) 

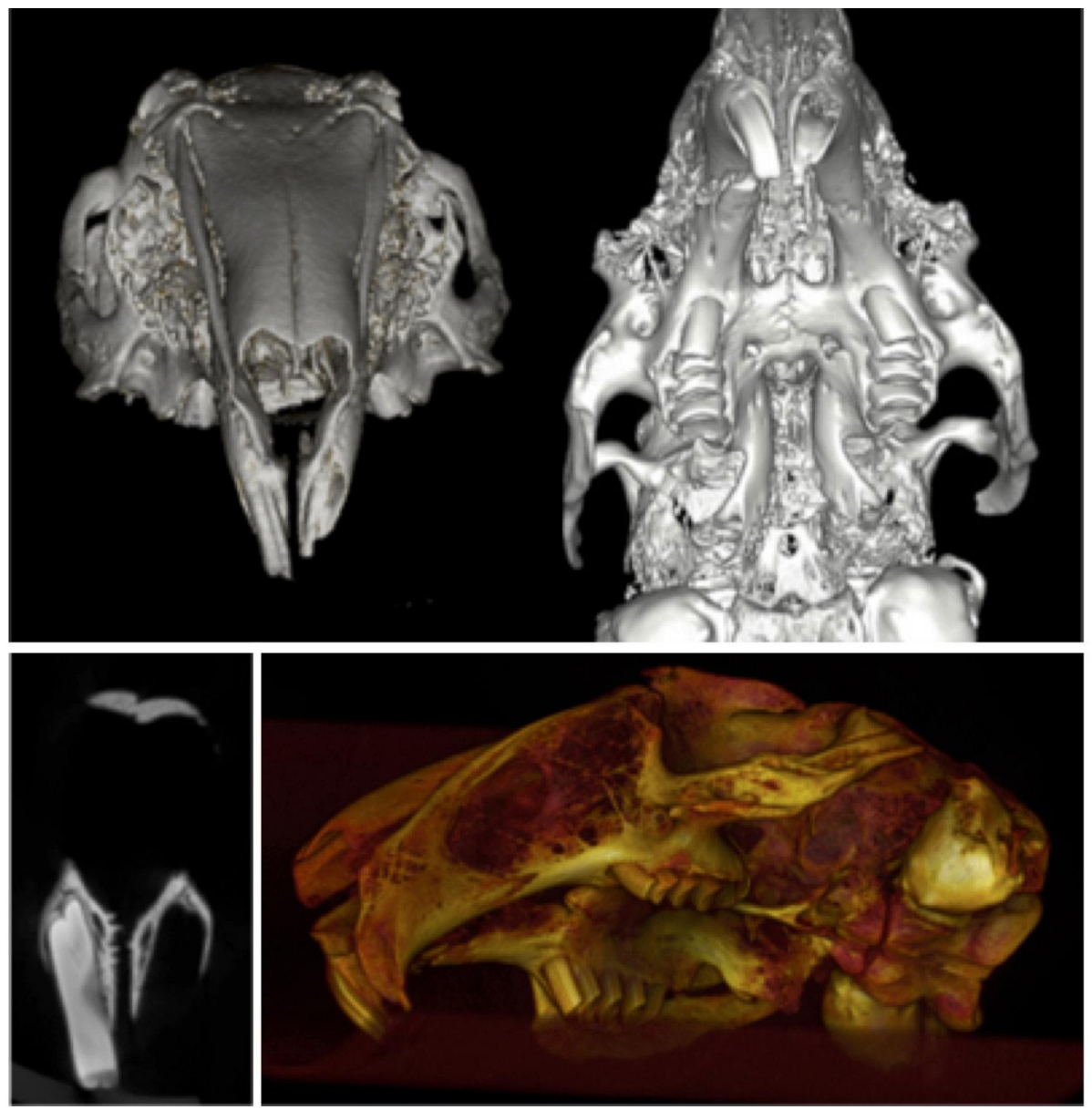

Micro-CT three-dimensional reconstruction of a preserved skull of a NZW rabbit showing the depth and morphology of the extraction socket. Coronal sections at the level of the central incisors show thin bony plates separating the tooth from the nasal cavity.

\section{In-vivo alveolar cleft creation surgery: Animals, Anesthesia, and Housing}

After evaluation of the correct position and volume of the cleft in the post-mortem pilot sixteen 8-week-old New Zealand White rabbits weighing 2.6 to 3.0 kilograms were operated in the same manner to prove the model in an in vivo setting. The procedure was conducted at the Animal Research Centre, Health Sciences Centre, Kuwait University. The project was subjected to strict animal testing protocol and the approval by the animal ethical committee at Animal Research Centre, Health Sciences Centre, Kuwait University. The rabbits were sedated thirty minutes prior to the experimental surgery with Xylazine $\mathrm{HCl} 5$ $\mathrm{mg} / \mathrm{kg}$ by intramuscular injection and subsequently anesthetized by intravenous injection 
of $35 \mathrm{mg} / \mathrm{kg}$ of ketamine $\mathrm{HCl}$. To ensure a high standard of animal care, a veterinarian was administering the sedation, anesthesia, and care-taking of the animals following an already used methodology. 35, 36 The animals were kept in separate cages and fed pellets and water throughout the duration of the study. The rabbits were cared of accordingly per protocol and observed by veterinarian until the end of the study.

Figure 3: Postmortem pilot alveolar cleft creation surgery.
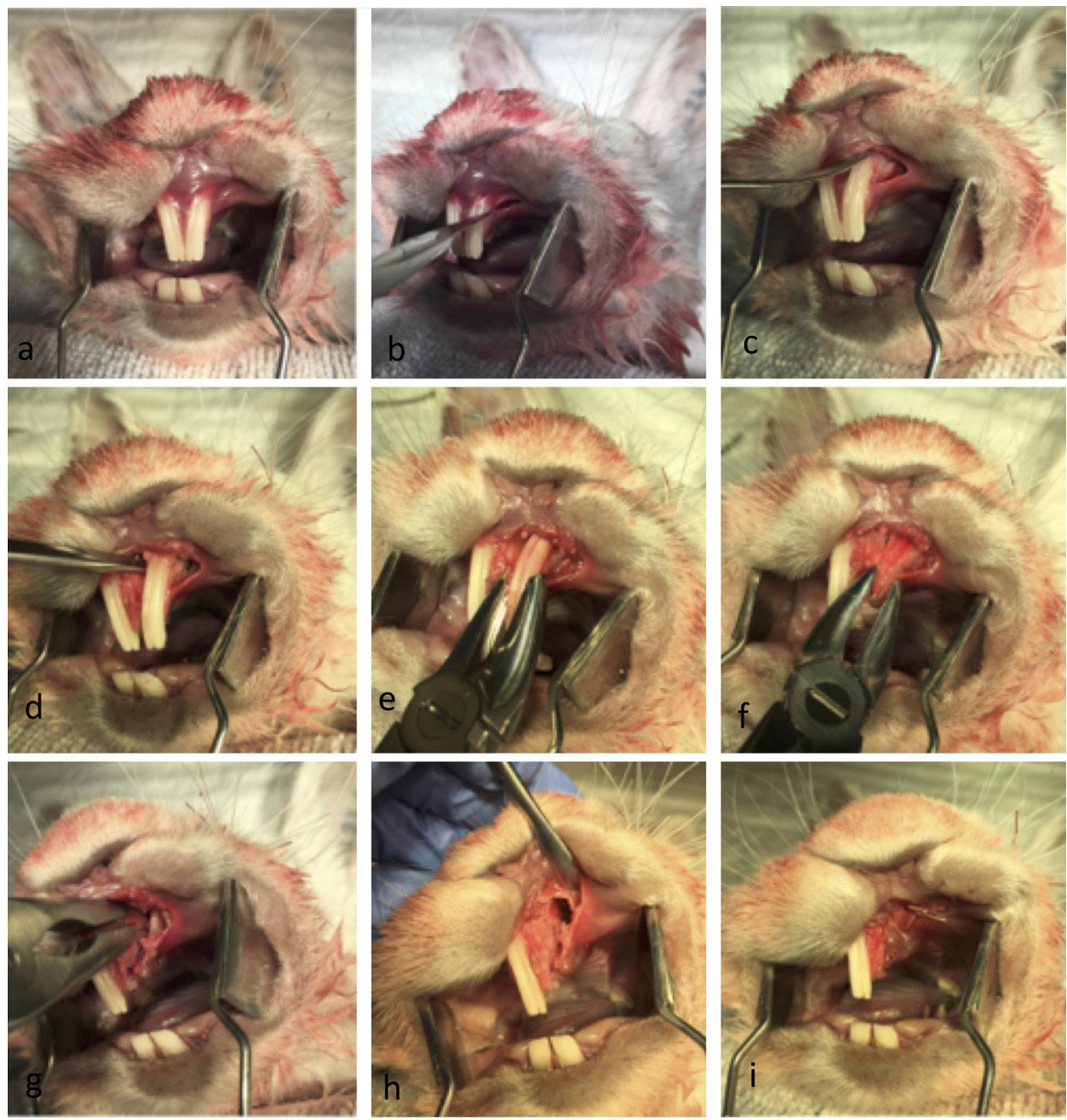

(a) to (i) Postmortem pilot alveolar cleft creation surgery of a sacrificed NZW rabbit showing the modification of the extraction socket to expose the nasal lining. The defect can be completely covered by mucosa to allow healing and enable mucosal coverage of the cleft surfaces. 


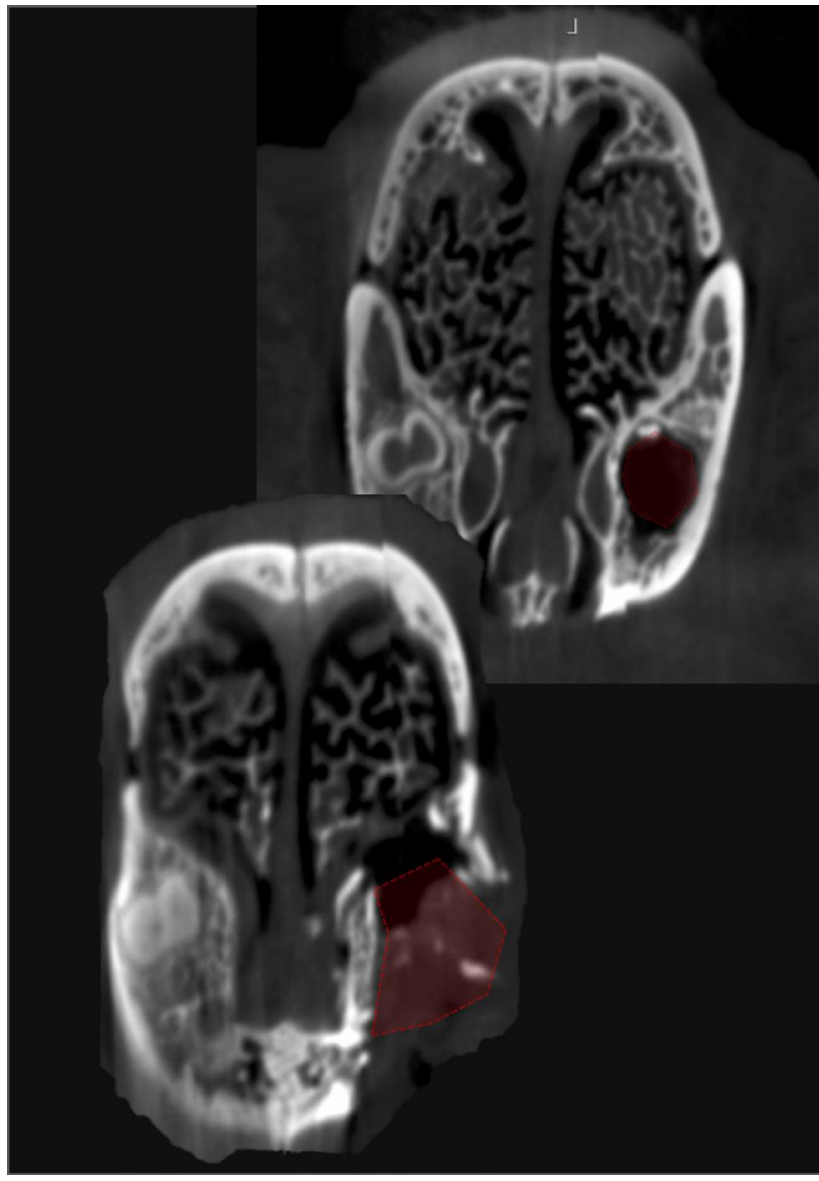

Micro-CT imaging of the created cleft in a pilot postmortem surgery. Coronal view at the level of the anterior part of the extraction socket shows a complete cleft $8 \mathrm{~mm}$ wide extending from the oral cavity to the nasal cavity. Coronal view at the apical region of the extracted central incisor shows a residual defect with all bony walls intact.

\section{In-vivo alveolar cleft creation surgery in NZW rabbits $(n=16)$}

Preparation of surgical sites in Maxillary Alveolus:

The surgery was performed with the rabbits in a supine position and under sterile conditions. A linear mucosal incision approximately was performed $2 \mathrm{~cm}$ lateral to the left central incisor and along the curvature of the tooth, extending to the distobuccal angle of the left central incisor, and then extended into the gingival margin of the left central incision on the facial aspect to the midline papilla. The gingiva and soft tissue were to expose the maxillary alveolus and the periodontal attachment of the central incisor tooth. Subsequently a flap was raised subperiosteally to expose the inferior nasal aperture. The nasal mucosa was freely protected and elevated using curved periosteal elevator taking 
care not to perforate the nasal mucosa. Lateral osteotomy along the lateral curvature of the left central incisor was performed using a rotary instrument with a round carbide bur to create a window exposing the root of the central incisor. The central incisor was then gently luxated toward the weakened lateral wall using a small dental elevator and eventually extracted using a veterinarian dental extraction forceps for rodents. Further osteotomy was carried to remove the superior and inferior bony plates with rongeur forceps to expose the nasal mucosa without injuring the mucosa. Bone wax then applied to the bony walls of the defect and the oral mucosa was approximated and sutured with 5 zeros resorbable Vicryl suture (Vicryl, Ethicon, USA) only on the medial and lateral sides. The central part of the wound was left open creating a pocket overlooking the bony defect. The defect was packed at the end with oxidised cellulose (Surgicel, USA). The nasal mucosa was left intact throughout the surgical procedure. The animals were allowed a period of 8 weeks for healing of the defect and the creation of maxillary alveolar defect.

The same surgical preparation and the strict protocol was carried at the second stage to expose the alveolar defect in preparation of bone grafting. A submarginal incision is made in the alveolar defect to separate the oral from the nasal mucosa. The Bony defect was exposed and bony walls were lightly freshened with a round diamond bur. The rabbits were fed soft diet ad lib directly after the surgery. The rabbits were cared of accordingly per protocol and watched by veterinarian until the end of the study after 3 months.

\section{Post-operative Cone Beam Computed Tomography of the alveolar clefts:}

Postoperative imaging with Cone Beam CT of the rabbit skull was obtained 8 weeks after cleft creation surgery. (Figure 8) 
Figure 5: In-vivo alveolar cleft creation surgery.
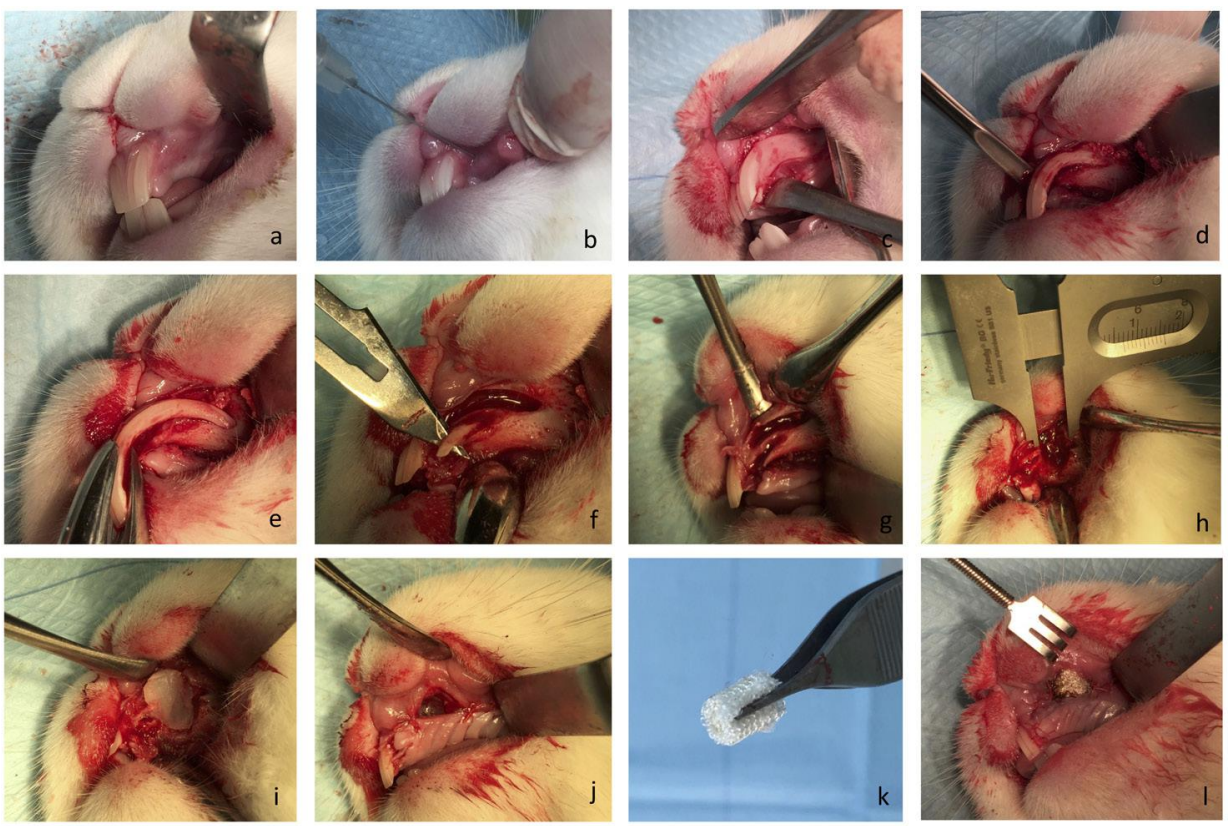

(a) to (I) In-vivo cleft creation surgery showing the modification of the extraction socket to expose the nasal lining. Distance material of bone wax and oxidized cellulose were used to keep the defect open and allow the mucosa to cover the cleft surfaces. 


\section{RESULTS}

\section{Osteological survey of New Zealand White rabbit skull (Oryctolagus cuniculus):}

A visual inspection of a skull of NZW rabbit revealed that in the area of interest to our study, the anterior maxilla of a NZW rabbit harboured two paired central incisors and two paired accessory palatal incisors. The central incisor was prominent and is in the form of half a circle and the accessory palatal incisor was smaller and roughly half the length of the central incisor. Examining a skull with an extracted central incisor revealed that the prominent tooth traversed the maxillary bone just below the nasal aperture leaving a thin layer of bone separating the alveolar socket from the nasal mucosa. The alveolar socket of an extracted central incisor formed a pocket-like cavity with a dimention of 7-8 mm made it an ideal model for alveolar cleft studies once the superior and inferior bony plate was resected to create a continuous defect simulating what is commonly seen in patients with congenital alveolar cleft. (Figure 1)

\section{Microfocus Computed Tomography (Micro-CT) for imaging survey:}

Three-Dimessional Micro-CT reconstruction of the rabbit skull and the Micro-CT imaging revealed high level of accuracy and clear view of the maxillary alveolar socket for volume analysis and measurement of bony landmarks. (Figure 2)

\section{Postmortem pilot alveolar cleft creation surgery and Micro-CT imaging:}

Simulation of the alveolar cleft surgery on a sacrificed rabbit skull revealed the feasabilty of creating an adequately sized defect. The defect could be easily extended to the nasal mucosa to simulate a real clinical defect. Soft tissue closure and suturing of the mucosa was readily performed. (Figure 3) Three-Dimessional reconstruction of the defect revealed a triangular alveolar cleft defect with a width of $8 \mathrm{~mm}$ that extended to the nasal cavity with the apex of the defect posting posteriorly and toward the depth of the alveolar socket. This simulates the clinical findings in patients with alveolar cleft defect. (Figure 4)

\section{In-vivo alveolar cleft creation surgery in NZW rabbits}

The surgery for each rabbit took between 15 to 30 min, and all procedures could be carried out during Ketamine anesthesia without endotracheal intubation. (Figure 5) In a few cases an extra injection of Ketamine was required. Bleeding was minor and not a clinical issue during or after the cleft creation surgery. The animals were active and behaved adequately immediately after the surgery. They all started eating already during the first days after surgery. The animals were fed ad libitum well throughout the study. All the rabbits survived during the 8 weeks postoperatively until day of the sacrifice. (Figure 6 and 7) The methodology and results of this in vivo-study will be reported more in detail in later separate papers. 
Figure 6: Wound healing.
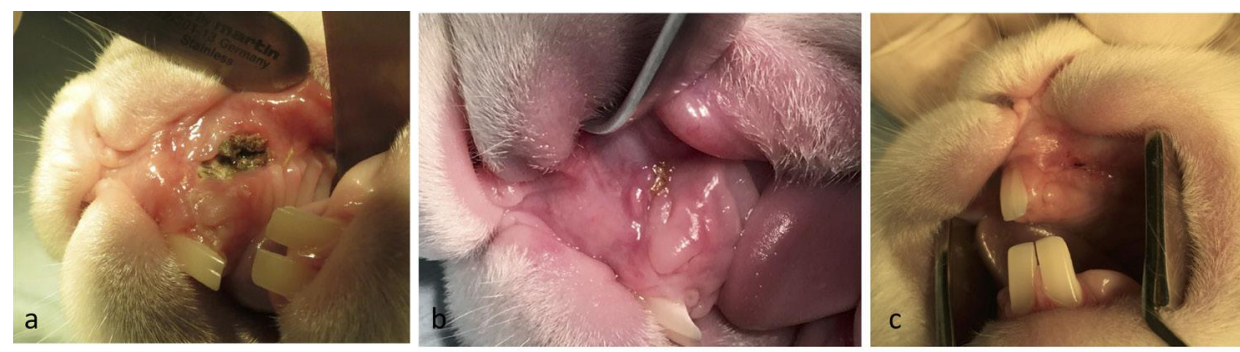

Healing of the surgery site one week after surgery (a) and 3 weeks after surgery (b). (c) Healed cleft site 8 weeks after surgery and before exposure of the alveolar cleft for bone grafting. Note the fistula overlying the cleft side indicating the establishment of an underlying bone defect.

Figure 7: Grafting of the defect.
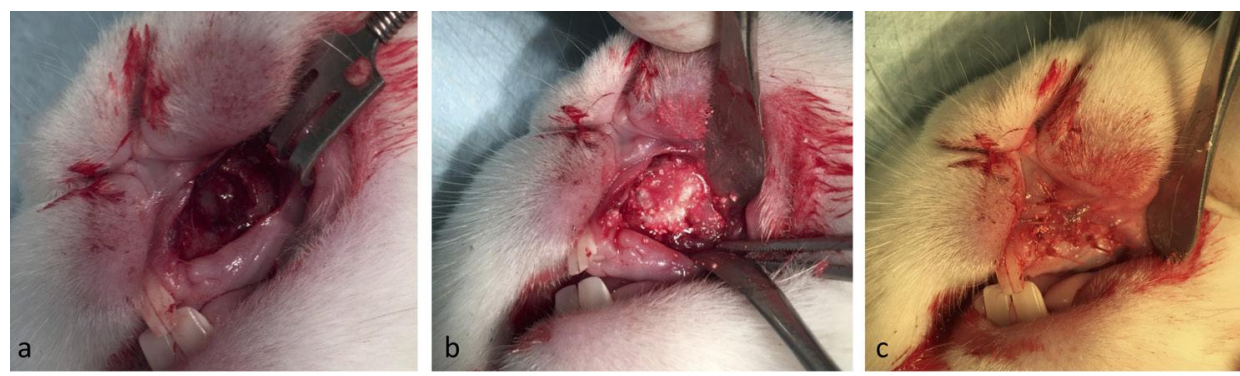

(a) Healed alveolar cleft site 8 weeks after surgery and before grafting intervention. (b) Grafting intervention in the established alveolar cleft. (c) Final coverage of the cleft with oral mucosa. Complete coverage of the defect is achieved without wide mobilization of the soft tissue.

\section{Post-operative Computed Tomography of the alveolar clefts:}

After 8 weeks healing, computed tomography was carried out in one animal. A three-dimensional reconstruction of the defect revealed a triangular alveolar cleft defect with a width of $8 \mathrm{~mm}$ and extending to nasal cavity with the apex of the defect posting posteriorly and toward the depth of the alveolar socket was verified. (Figure 8) 


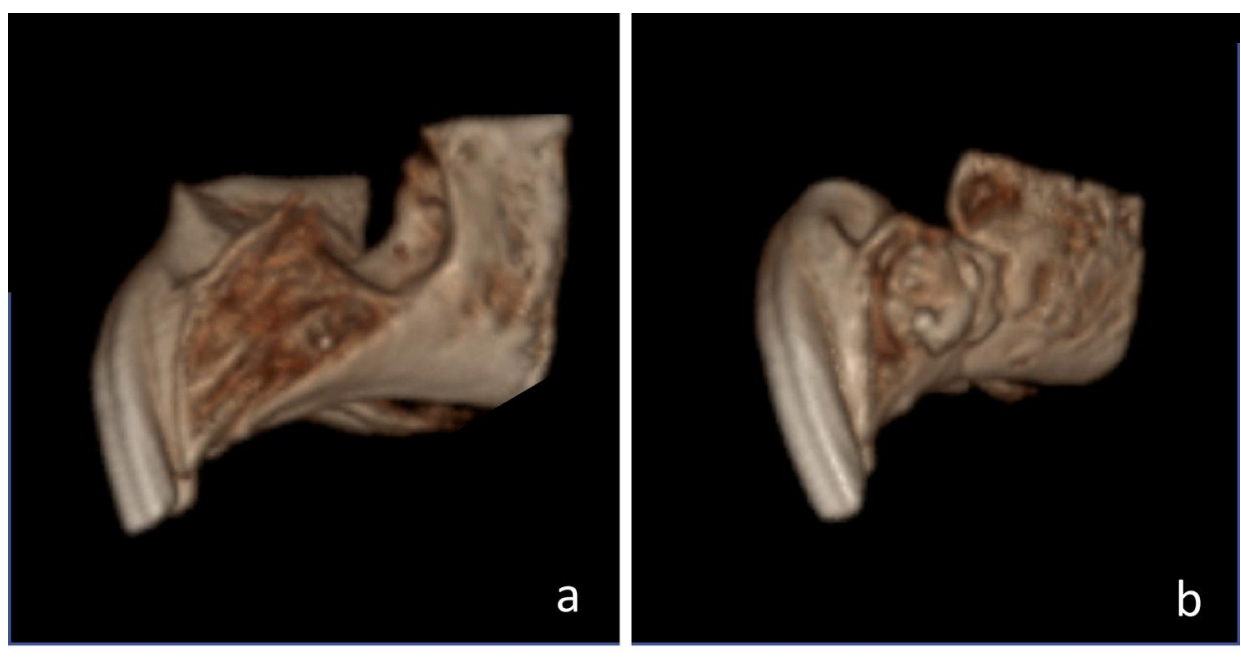

(a) Cone Ceam CT imaging of the created alveolar cleft after 8 weeks of healing shows a complete cleft extending from the oral cavity to the nasal cavity. (b) Alveolar cleft 8 weeks after grafting the defect with bone substitute material. 


\section{DISCUSSION}

Alveolar maxillary defects are unique defects with regard to their overlying soft tissues; the oral mucosa inside the mouth and the nasal mucosa as the nasal floor lining. The topography of such defect makes it susceptible to two biologically distinctive anatomical compartments each with a particular bio-environment. The aim of alveolar bone grafting is to obtain a proper reestablishment of these anatomical structures and provide a new bone structure that are acceptable in regard to volume and bone quality. Utilization of an animal model with a healed alveolar cleft defect, which mimics the three dimensional morphology in human patients with cleft lip and palate and extending to the nasal mucosa, would be best model to test the healing pattern of bone graft materials and the establish the proper anatomical structure.

Proper grafting of alveolar cleft deformities is an essential step to re-establish the dental arch in patients with cleft palate. This depends on the type of the grafting material that aims to restore form, volume and the functional establishment of a skeletal biological medium that would allow the eruption of the permanent teeth. Recent advances in tissue-engineered bone substitute materials and biomedical science have prompt further improvements in existing testing animal models to better evaluate the osteogenic efficacy and healing efficiency of the new grafting materials. ${ }^{4-8,37}$

Several models have been proposed as alveolar cleft model for testing of tissue-engineered bone replacement material. These ranging from mice, rats, rabbits, cats, dogs, swines, goats, sheep and monkeys. 4, 9-24 Prior description on rats models were able to create defects simulating alveolar defects because of their ease of handling and cost effectiveness, however, these defects tend to be significantly smaller in volume than human alveolar defects making it technically challenging to properly perform the grafting testing procedure. $13,17,18,21,24$

The first description in the litature on an animal model for creating an alveolar cleft was reported by Harvold. He described the creation of an alveolar and palatal cleft $2 \mathrm{~mm}$ wide in 2 Rhesus monkeys. ${ }^{38}$ The skeletal metabolism and bony macrostructure is small rodents tends to be far more active than bigger mammals and this may compromise the applicability of the animal testing findings. Thus bigger animals were considered a closer models to the human counterparts and able of mimicking skeletal defects and several attempts have been trying to describe further surgical techniques and modifications on previously reported methodologies mostly on monkeys and dog models. ${ }^{11,12}$ In recent years, attempts have been made to create more effective, economical and smaller animals to create acceptable alveolar cleft defects. Despite the increased descriptions of several animal models for alveolar 
bone grafting materials testing, most of the in vivo animal models using small animals were not able to function as reliable alveolar cleft model simulating that in human. This is mostly because of the limited anatomical size in these models which lead to difficulties in handling intraoral surgical procedures. This results in a compromise in designing and establishing proper sizable defects mimicking three-dimensional triangular defect extending to the nasal mucosa and the adjacent tooth as seen in human patients. ${ }^{12,18,39}$

Most current reports in the literature illustrated the creation of simple non-anatomical defects in the maxilla of small animals by creating a maxillary or palatal window to establish a communication between the oral cavity and the nasal cavity, as described by Nguyen et. al., Raposo-Amaral et. al., Mostafa et. al., Takano-Yamamoto et. al. and Kim et. al. in rats, and by Sawada et. al. and Puumanen et. al. in rabbits. ${ }^{17,18,21,22,39-41}$ Xu et. al. described the establishment of a cleft model in rats by extracting a molar tooth and applying bone wax. ${ }^{24}$ Their model has succeeded in controlling the osseos healing process but the anatomical location of the defect does not correspond to a tridimentional maxillary alveolar cleft defects as encountered clinically. In some studies the defects were created and were not allowed to heal to establish a true non-healing critical defect and the insertion of the grafting material was performed simultaneously during the cleft creation surgery as seen in the models described by Takano-Yamamoto et. al, Sawada et. al., Puumanen et. al., Pilanci, and Kim et. al. ${ }^{20,22,39-41}$ The immediate grafting of the created defect leads to masking the effect of the native bone healing, and thus it would be difficult to attribute the new bone formation to the potential of the inserted graft rather than the native bone healing. El-Bokle et. al. described a rabbit model for creating an alveolar cleft defect by extracting the central and lateral incisor and creating a wide defect and splitting the nasal mucosa to suture it the oral mucosa and leaving $1 \mathrm{~cm}$ defect. ${ }^{11}$ In our opinion, a wide skeletal defect is plausible in creating alveolar cleft but $1 \mathrm{~cm}$ oronasal communication rarely correlates with clinical scienarios. A meta-analysis by Bykowski et. al. evaluated the rate of oronasal fistula after primary cleft repair surgery and reported that most of the significant oro-nasal fistulas leading to clinical symptoms occurred posterior to the incisive foramen with the highest incidence at the soft-hard palate junction, and the lowest incidence of the fistula occurring at the maxillary alveolus. ${ }^{42}$ In addition, splitting and suturing the nasal mucosa in a rabbit model is challenging given the anatomical limitation and would significantly incerase the operating time and may compromise the health of the animal due to incerased anesthetic requirement, compromise oral intake postoperatively, and increase the risk of aspiration and bleeding into the nasal cavity.

In our model we were able to achieve a reliable and consistent alveolar cleft in the maxillary front region and extending to the nasal mucosa, along with a nearby tooth which simulates the local anatomy of maxillary alveolar cleft in cleft lip and palate patients. Extracting an 
incisor by removing bone lateral to the incisor and luxate the tooth laterally has recently been described by Maslamani et al in an experiemental tooth replantation study. ${ }^{43}$ Due to the extremely curved rabbit incisor it is not possible to extract through the longitudinal axis of the tooth but instead through lateral luxation after some bone removal.

Preparation of the cleft by keeping the nasal mucosa intact was possible and the surgery can be carried in 15-20 minutes and without general inhalation anesthesia or intubation, and without major risk of aspiration. The control of the space preventing it from bony ingrowth with simple bone wax seems to be consistently efficent in limiting bone healing, and the oxidized cellulose help in maintaing a spaced and aiding in prevent the collapse of the defect by extensive fibrous tissue. Allowing 8 weeks for cleft creation surgery seems to be enough to assume critically sized defect.

Our study shows that it is possible to produce a reliable and predictable animal model in rabbits to perform alveolar bone grafting. The surgical site area is of similar size and in the same region as that of pediatric population in human subjects, and the procedure does not require magnifying apparatus or micro instruments.

\section{CONCLUSIONS}

A simple and predictable rabbit model with alveolar cleft for the clinical testing of e.g. tissue-eingineering bone substitute materials can be created by following the existing anatomy and extraction of a central incisor tooth, modification of the extraction socket by extending it to the nasal mucosa, and the application of simple bone wax and oxidized cellulose material to help modulating the healing phase in the cleft area and avoid rapid bone generation and filling of the defect. Allowing 8 weeks of healing yields a predictable and good sized defect that can be used for later grafting procedures.

\section{ABBREVATIONS:}

NZW: New Zealand White rabbit.

\section{AUTHORS' CONTRIBUTIONS:}

$\mathrm{MK}, \mathrm{BL}$, and PK designed the study. MK, AB and RT performed the post-mortem procedure and defined the protocol. MK, LA, and $B L$ were responsible for the in-vivo surgery 
and performing the procedure. FG was responsible for imaging acquisition and analysis. MK and BL prepared the manuscript. RT, FH, PK, and LA were responsible for revising the manuscript critically for important intellectual content. All Authors read and approved the final manuscript.

\section{ACKNOWLEDGEMENTS:}

The authors are grateful to Dr Severino Gabato for assisting in the in-vivo surgery and taking excellent care of the animals. We are also grateful to Animal Resources Center Health Sciences Center for providing postmortem material for the pilot study.

\section{COMPETING INTERESTS:}

The authors report no conflicts of interest related to this study.

\section{AVAILABILITY OF DATA AND MATERIAL:}

The data/material analysed during the current study available from the curresponding author on request.

\section{CONSENT FOR PUBLICATION:}

Not applicable

\section{ETHICS APPROVAL AND CONSENT TO PARTICIPATE:}

All animals were handled in compliance with European Communities Council Directive of 24 November 1986 (86/609/EEC) for the care of laboratory animals in experimental procedures and ethical guidelines for animal research. ${ }^{44}$ All methods were carried out in accordance with the approved guidelines and regulations of the HSC Ethical Committee for the use of Laboratory Animals in Teaching and in Research, Kuwait University. 
Chapter 6

\section{FUNDING:}

This study was supported by the resources of Health Sciences Centre, Kuwait University and RWTH Aachen University, Germany. 


\section{REFERENCES}

1. Rychlik D, Wójcicki P, Koźlik M. Osteoplasty of the alveolar cleft defect. Adv Clin Exp Med2012. p. 255-62.

2. Santiago PE, Schuster LA, Levy-Bercowski D. Management of the alveolar cleft. Clin Plast Surg2014. p. 219-32.

3. Seifeldin SA. Is alveolar cleft reconstruction still controversial? (Review of literature). Saudi Dent J2016. p. 3-11.

4. Chung VH, Chen AY, Jeng LB, Kwan CC, Cheng SH, Chang SC. Engineered autologous bone marrow mesenchymal stem cells: alternative to cleft alveolar bone graft surgery. The Journal of craniofacial surgery. 2012;23:1558-63.

5. Gładysz D, Hozyasz KK. Stem cell regenerative therapy in alveolar cleft reconstruction. Arch Oral Biol2015. p. 1517-32.

6. Janssen NG, Weijs WLJ, Koole R, Rosenberg AJWP, Meijer GJ. Tissue engineering strategies for alveolar cleft reconstruction: a systematic review of the literature. Clin Oral Investig: Springer Berlin Heidelberg; 2014. p. 219-26.

7. Kawata T, Kohno S, Fujita T, Sugiyama H, Tokimasa C, Kaku M, et al. New biomaterials and methods for craniofacial bone defect: chondroid bone grafts in maxillary alveolar clefts. Journal of craniofacial genetics and developmental biology. 2000;20:49-52.

8. Khojasteh A, Kheiri L, Motamedian SR, Nadjmi N. Regenerative medicine in the treatment of alveolar cleft defect: A systematic review of the literature. Journal of cranio-maxillo-facial surgery : official publication of the European Association for Cranio-Maxillo-Facial Surgery2015. p. 1608-13.

9. Caballero M, Morse JC, Halevi AE, Emodi O, Pharaon MR, Wood JS, et al. Juvenile Swine Surgical Alveolar Cleft Model to Test Novel Autologous Stem Cell Therapies. Tissue Eng Part C Methods: Mary Ann Liebert, Inc. 140 Huguenot Street, 3rd Floor New Rochelle, NY 10801 USA; 2015. p. 898-908.

10. de Ruiter A, Meijer G, Dormaar T, Janssen N, van der Bilt A, Slootweg P, et al. $\beta$-TCP versus autologous bone for repair of alveolar clefts in a goat model. Cleft Palate Craniofac J: Allen Press Publishing Services; 2011. p. 654-62.

11. el-Bokle D, Smith SJ, Germane N, Sharawy M. New technique for creating permanent experimental alveolar clefts in a rabbit model. Cleft Palate Craniofac J1993. p. 542-7.

12. El-Deeb M, Horswell B, Waite DE. A primate model for producing experimental alveolar cleft defects. J Oral Maxillofac Surg1985. p. 523-7.

13. Gritli-Linde A. The mouse as a developmental model for cleft lip and palate research. Front Oral Biol: S. KARGER AG; 2012. p. 32-51.

14. Ishii Y. [Experimental study of secondary bone graft of alveolar clefts using bone morphogenetic protein (BMP)]. Kokubyo Gakkai zasshi The Journal of the Stomatological Society, Japan. 2001;68:111-24.

15. Liang L, Liu C. Trans-sutural distraction osteogenesis for alveolar cleft repair: an experimental canine study. Cleft Palate Craniofac J: Allen Press Publishing Services; 2012. p. 701-7.

16. Liao LS, Tan Z, Zheng Q, Wu J, Shi B, He X, et al. Animal experimental study on repairing alveolar clefts by using rectilinear distraction osteogenesis. Journal of plastic, reconstructive \& aesthetic surgery : JPRAS. 2009;62:1573-9.

17. Mostafa NZ, Doschak MR, Major PW, Talwar R. Reliable critical sized defect rodent model for cleft palate research. Journal of cranio-maxillo-facial surgery : official publication of the European Association for Cranio-Maxillo-Facial Surgery2014. p. 1840-6.

18. Nguyen PD, Lin CD, Allori AC, Ricci JL, Saadeh PB, Warren SM. Establishment of a critical-sized alveolar defect in the rat: a model for human gingivoperiosteoplasty. Plastic and reconstructive surgery. 2009;123:817-25. 
19. Papadopoulos MA, Papadopulos NA, Jannowitz C, Boettcher P, Henke J, Stolla R, et al. Three-dimensional cephalometric evaluation of maxillary growth following in utero repair of cleft lip and alveolar-like defects in the mid-gestational sheep model. Fetal diagnosis and therapy. 2006;21:105-14.

20. Pilanci O, Cinar C, Kuvat SV, Altintas M, Guzel Z, Kilic A. Effects of Hydroxyapatite on Bone Graft Resorption in an Experimental Model of Maxillary Alveolar Arch Defects. Archives of Clinical and Experimental Surgery (ACES). 2013;2:170-5.

21. Raposo-Amaral CE, Kobayashi GS, Almeida AB, Bueno DF, Freitas FRdSe, Vulcano LC, et al. Alveolar osseous defect in rat for cell therapy: preliminary report. Acta Cir Bras2010. p. 313-7.

22. Sawada Y, Hokugo A, Nishiura A, Hokugo R, Matsumoto N, Morita S, et al. A trial of alveolar cleft bone regeneration by controlled release of bone morphogenetic protein: an experimental study in rabbits. Oral surgery, oral medicine, oral pathology, oral radiology, and endodontics. 2009;108:812-20.

23. Wu L-I, Zhao Y, Chen C. [Establishment of the animal model with unilateral alveolar cleft and its effect on the nose growth]. Zhonghua Zheng Xing Wai Ke Za Zhi2010. p. 39-42.

24. Xu Y, Sun J, Chen Z. Establishment of a rat model for alveolar cleft with bone wax. Journal of oral and maxillofacial surgery : official journal of the American Association of Oral and Maxillofacial Surgeons. 2015;73:733. e1-10.

25. Harling TR, Stelnicki EJ, Hedrick MH, Longaker MT. In utero models of craniofacial surgery. World journal of surgery. 2003;27:108-16.

26. Hedrick MH, Rice HE, Vander Wall KJ, Adzick NS, Harrison MR, Siebert J, et al. Delayed in utero repair of surgically created fetal cleft lip and palate. Plastic and reconstructive surgery. 1996;97:900-5.

27. Wenghoefer MHO, Deprest J, Goetz W, Kuijpers-Jagtman AM, Bergé S. Prenatal cleft lip and maxillary alveolar defect repair in a 2-step fetal lamb model. Journal of Oral and Maxillofacial Surgery. 2007;65:2479-86.

28. IGAWA HH, OHURA T, IWAO F, YAMAMOTO Y, FUJIOKA H. Intrauterine Repair of Cleft Lip in Mouse Fetuses. Congenital Anomalies. 1991;31:95-100.

29. Leitao JM, Pereira LA, Gonçalves FL, Schmidt AF, Sbragia L. The ideal timing for experimental cleft lip creation. The Cleft Palate-Craniofacial Journal. 2011;48:38-43

30. Schardein J, Petrere J, Hentz D, Camp R, Kurtz S. Cannibalistic traits observed in rats treated with a teratogen. Laboratory animals. 1978;12:81-3.

31. Li Y, Chen S-K, Li L, Qin L, Wang X-L, Lai Y-X. Bone defect animal models for testing efficacy of bone substitute biomaterials. Journal of Orthopaedic Translation. 2015;3:95-104.

32. Mapara M, Thomas BS, Bhat K. Rabbit as an animal model for experimental research. Dental research journal. 2012;9.

33. Pearce A, Richards R, Milz S, Schneider E, Pearce S. Animal models for implant biomaterial research in bone: a review. Eur Cell Mater. 2007;13:1-10

34. Wang X, Mabrey JD, Agrawal CM. An interspecies comparison of bone fracture properties. Biomedical Materials and Engineering. 1998;8:1-10

35. Al-Asfour A, Andersson L, Kamal M, Joseph B. New bone formation around xenogenic dentin grafts to rabbit tibia marrow. Dental traumatology : official publication of International Association for Dental Traumatology. 2013;29:455-60.

36. Andersson L, Ramzi A, Joseph B. Studies on dentin grafts to bone defects in rabbit tibia and mandible; development of an experimental model. Dental traumatology : official publication of International Association for Dental Traumatology. 2009;25:78-83.

37. KolkA, Handschel J, Drescher W, Rothamel D, Kloss F, Blessmann M, et al. Current trends and future perspectives of bone substitute materials - from space holders to innovative biomaterials. Journal of cranio-maxillo-facial surgery : official publication of the European Association for Cranio-Maxillo-Facial Surgery. 2012;40:706-18.

38. Harvold E. Cleft palate, an experiment. Acta Odontologica Scandinavica. 1950;9:84-7. 
39. Kim J-H, Moon H-J, Kim T-H, Jo J-M, Yang SH, Naskar D, et al. A novel in vivo platform for studying alveolar bone regeneration in rat. Journal of tissue engineering. 2013;4:2041731413517705.

40. Puumanen K, Kellomaki M, Ritsila V, Bohling T, Tormala P, Waris T, et al. A novel bioabsorbable composite membrane of Polyactive 70/30 and bioactive glass number 13--93 in repair of experimental maxillary alveolar cleft defects. Journal of biomedical materials research Part B, Applied biomaterials. 2005;75:25-33.

41. Takano-Yamamoto T, Kawakami M, Sakuda M. Defects of the rat premaxilla as a model of alveolar clefts for testing bone-inductive agents. J Oral Maxillofac Surg1993. p. 887-91.

42. Bykowski MR, Naran S, Winger DG, Losee JE. The rate of oronasal fistula following primary cleft palate surgery: a meta-analysis. The Cleft Palate-Craniofacial Journal. 2015;52:e81-e7.

43. Maslamani M, Almusawi A, Joseph B, Gabato S, Andersson L. An experimental model for studies on delayed tooth replantation and ankylosis in rabbits. Dental traumatology : official publication of International Association for Dental Traumatology. 2016;32:443-9.

44. Directive C. 86/609/EEC of 24 November 1986 on the approximation of laws, regulations and administrative provisions of the Member States regarding the protection of animals used for experimental and other scientific purposes. Off J Eur Commun. 1986;29:L358. 


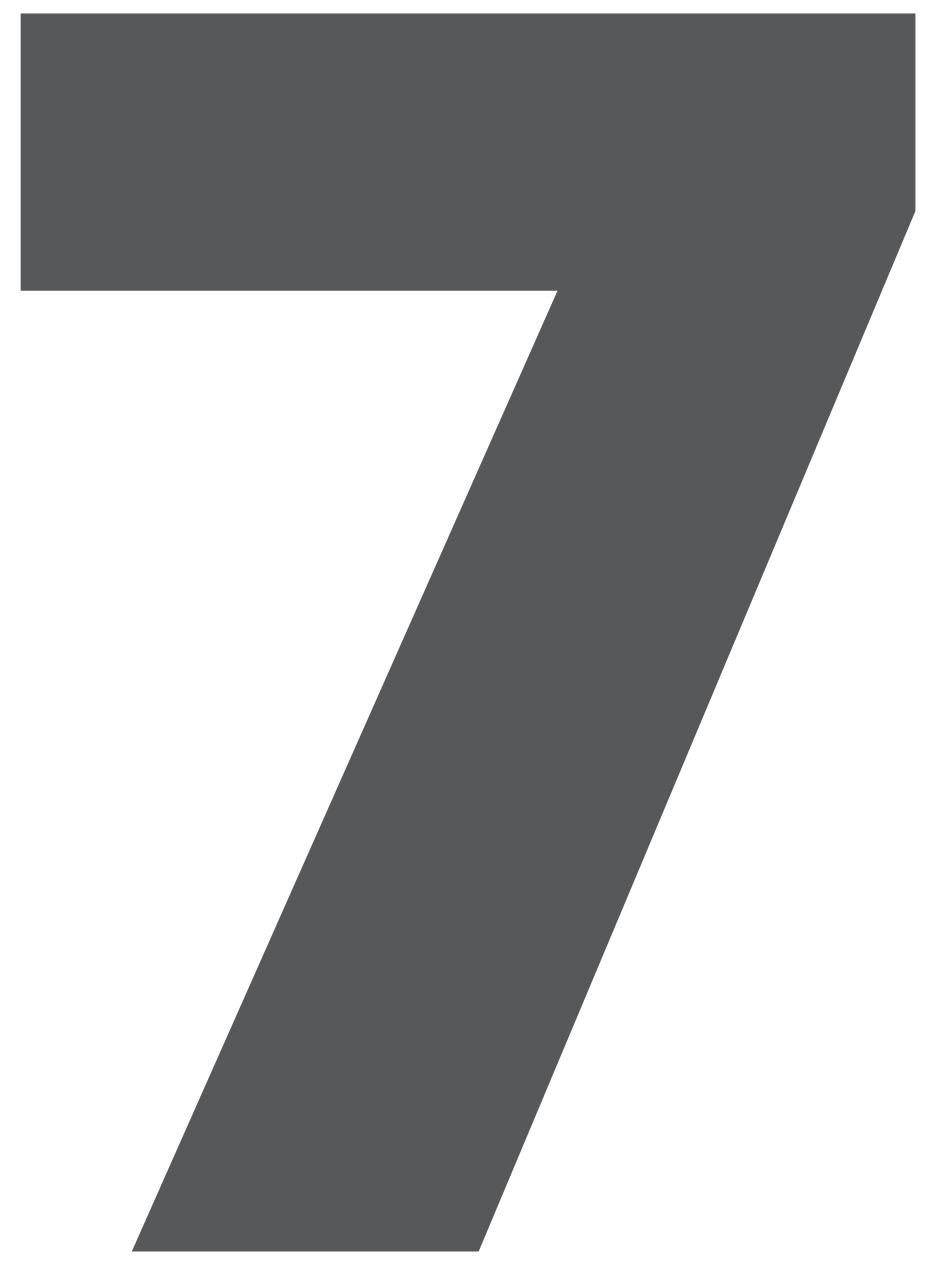




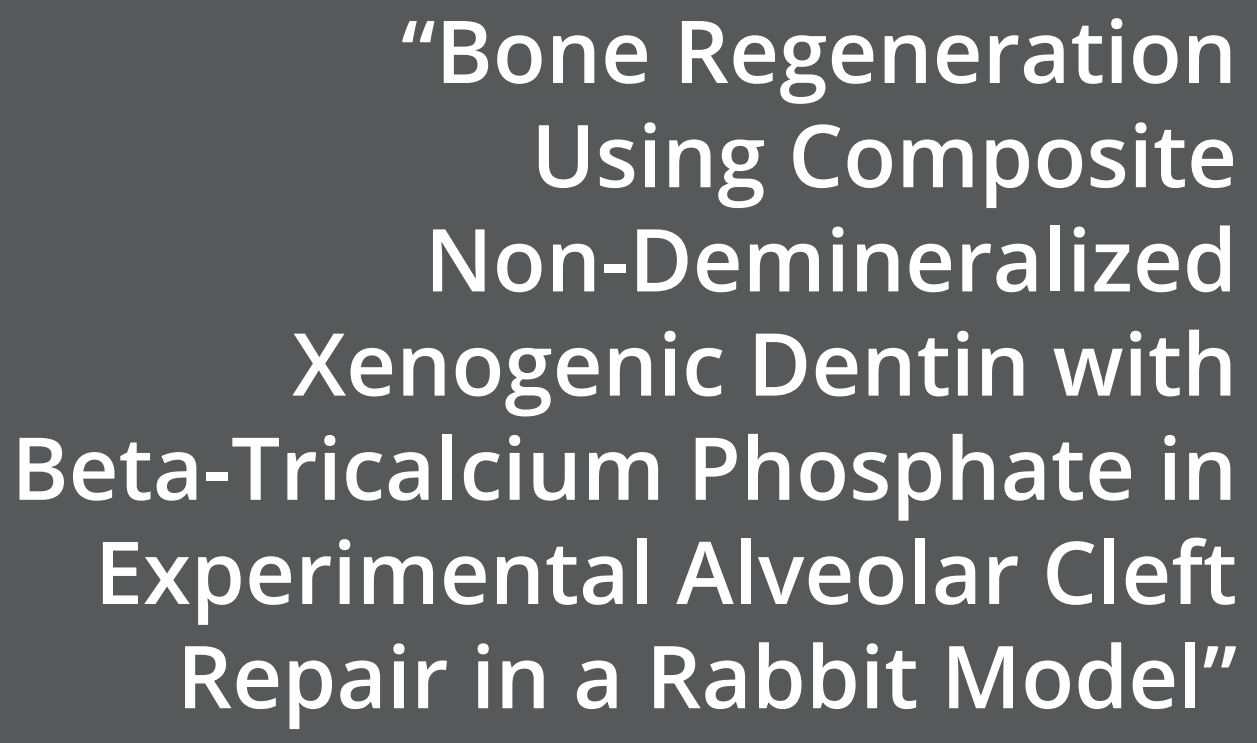

Mohammad Kamal; Lars Andersson; Rene Tolba; Adel Al-Asfour;

Alexander K. Bartella; Felix Gremse; Stefanie Rosenhain; Frank Hölzle; Peter Kessler; Bernd Lethaus

Published in the Journal of Translational Medicine. 2017 Dec 23;15(1):263. doi: 10.1186/s12967-017-1369-3 


\section{ABSTRACT}

Background: Alveolar cleft repair is performed via bone grafting procedure to restore the dental arch continuity. A suitable bone substitute material should possess osteoinductive and osteoconductive properties, to promote new bone formation, along with a slowly resorbable scaffold that is subsequently replaced with functionally viable bone. Calcium phosphate biomaterials have long proved their efficacy as bone replacement materials. Dentin in several forms has also demonstrated its possibility to be used as bone graft replacement material in several studies. The purpose of this study was to evaluate bone regeneration pattern and quantify bone formation after grafting pre-established expimental alveolar clefts defects model in rabbits using composite xenogenic dentin and B-TCP in comparison to ß-TCP alone.

Methods: Unilateral alveolar cleft defects were created in 16 New Zealand rabbits according to previously described methodology. Alveolar clefts were allowed 8 weeks healing period. 8 defects were filled with B-TCP, whereas 8 defects filled with composite xenogenic dentin with B-TCP. Bone regeneration of the healed defects was compared at the 8 weeks after intervention. Quantifiation of bone formation was analyzed using Micro-computed tomography $(\mu \mathrm{CT})$ and histomorphometic analysis.

Results: $\mu C T$ and histomorphometric analysis revealed that defects filled with composite Dentin/ß-TCP showed statistically higher bone volume fraction, bone mineral density and percentage residual graft volume wgeb compared to ß-TCP alone. An improved surgical handling of the composite dentin/ß-TCP graft was also noted.

Conclusions: Composite xenogenic Dentin/ß-TCP putty expresses enhanced bone regeneration compared to ß-TCP alone in the reconstruction of rabbit alveolar clefts defects. 


\section{BACKGROUND}

Maxillary alveolar cleft is a congenital malformation occuring during fetal development leading to a compromised dental arch anatomy and disrupted sequnce teeth eruption. Alveolar cleft repair is performed during childhood via bone grafting procedure with the goal to restore the bony continuity of the dental arch, seal the oro-nasal communication, and create a favorable anatomy for dental rehabilitation. ${ }^{1-5}$ Alveolar bone cleft is rounitley grafted with autogneous bone obtained from various anatomical donor sites, however, autogenous bone is associated with inherent technical limitations such as donor site supply limitation and surgical morbidity, increased operative time and costs, and several clinical reports on its increased resorption rate with unpredictable graft stability. ${ }^{5-12}$

To overcome these shortcomings with autogenous bone, various regenerative bone substitute materials have been developed for skeletal grafting including processed xenogeneic bone ${ }^{13}$, allogenic bone ${ }^{14,15}$, several biodegradable alloplastic materials such as polymer based polyether ketone and fiber-reinforced bioactive glass materials 16,17 , and various degradable bioactive ceramics like tricalcium phosphate and hydroxyapetite. $6,9,11,12,15,18,19$

Biocompatible calcium phosphate ceramics have shown in in several human and animal studies to express osteoconductive properties leading to enhanced bone formation and the ability to function as a bone grafting substitute materials. 3, 14, 15, 17-20 Several types of calcium phosphate currently exist for clinical applications, with biphasic beta tri-calcium phosphate ( $\beta$-TCP) and calcium hydroxyapatite (HA) being the most common utilized. $\beta$-TCP and HA are mixed in a proportionate manner to combine the resiliency and improved mechanical properties offered by hydroxyapatite $(\mathrm{HA})$, along with the reliable biodegradability offered by tri-calcium phosphate ( $\beta$-TCP) to yield a suitable bone replacement materials with balanced clinical efficacy. 18,20

Dentin, a natural hard tissue component of teeth and similar in its chemical composition to bone, has likewise been explored as a bone replacement material. Several human and in-vivo studies have demonstrated the ability of dentin to promote bone formation due to its innate osteoconductive and osteoinductive properties. 13,21-28 The osteoconductive potential of dentin was evaluated in its non-demineralized form in an animal model by implanting 2-3 mm dentin blocks into bone marrow of rabbits, and subsequently leading bone formation close to the native tibia bone wall indicating the ability of the dentin blocks to induce osteogenesis. ${ }^{21}$ In addition to its known clinical effect in promoting bone formation and fusing with bone in a process of ankylosis as seen in several studies evaluating the replantation of avulsed teeth in human without the process of demineralization. ${ }^{23,24,29: 31}$ Several studies also demonstrated such onsteoinductivity potential of dentin when used in 
demineralized form, however, higher degree of dentin resorption rate and thus lower graft volume maintenance rate was report in these studies. ${ }^{22,27,32,33}$ Dentin has also been studied in various allogenic and xenogenic forms to explore its efficacy as bone replacement material including mineralized dried dentin as particulate chips and blocks, demineralized dentin, freeze-dried allogenic dentin, and processed bovine dentin. 13, 22, 25-28, 34

Despite recent biotechnological developments of bone substitute materials, optimising the quality of the existing regenerative materials and looking for novel and more effective materials is essential in developing a clinically suitable material. The establishment of a proper in-vivo biological model simulating alveolar clefts is essential in order to conduct experimental testing of bone grafting materials and evaluate the clinical effect with respect to osteogenesis and healing, and thus far several animal models have been developed in mice, rats, rabbits, cats, dogs, swines, goats, sheep and monkeys. 2, 6, 35-49

In a recent paper, an in-vivo rabbit model with surgically created alveolar clefts for the clinical testing was presented (45). The advantage with this model is that the cleft is kept open during the healing to avoid rapid bone generation and filling of the defect, enabling conditions similar to clefts seen in clinical practice. ${ }^{50}$

Giving the prevoiusly reported healing capacity of non-deminiralized dentin when used as bone replacement material, we wanted to investigate the effect of combining non-deminiralized dentin with the well-established B-TCP, and report on any ehancement in bone volume fraction, which may have caused by the adding dentin to ß-TCP. We propose that adding dentin would enhance the overall bone formation in the defect, including the fusion of the dentin particles to the healed graft though osseos integration with the newly formed bone, and undergoing a gradual and slow resorption process, thus maintaining a higher BV/TV ratio and improved graft volume maintenace rate. The aim of the current study was to evaluate bone regeneration potential and quantify the overall bone formation after grafting pre-established expimental alveolar clefts defects in a rabbit model using in-situ formed composite xenogenic non-demineralized dentin and ß-TCP in comparison to ß-TCP alone. 


\section{METHODS}

\section{Animals, Anesthesia, and Housing}

A New Zealand White rabbit model for alveolar cleft testing was previously described by the authors in detail (Figure 1). ${ }^{50}$ The experimental procedure was conducted at the Animal Research Centre, Health Sciences Centre, Kuwait University. The project was subjected to strict animal testing protocol and the approval by the animal ethical committee at Animal Research Centre, Health Sciences Centre, Kuwait University. Sixteen 8-weekold New Zealand White rabbits weighing 2.6 to 3.0 kilograms were operated in the same manner to prove the in-vivo model. The rabbits were sedated with Xylazine $\mathrm{HCl} 5 \mathrm{mg} / \mathrm{kg}$ by intramuscular injection thirty minutes prior to operation and subsequently anesthetized with $35 \mathrm{mg} / \mathrm{kg}$ of ketamine $\mathrm{HCl}$ by intravenous injection. To ascertain the highest standard of animal care, a veterinarian was administering the sedation, anesthesia, and care-taking of the animals according to previously used methodology. ${ }^{21,25,50}$ Throughout the duration of the study, rabbits were kept in separate cags and fed rabbit pellets and water mixture, and were cared of accordingly and observed by veterinarian until the completion of the study.

\section{Preparation of grafting material}

Human teeth, extracted for orthodontic reasons and then stored dry, were prepared by first removing the crowns then sectioning the roots vertically to expose the pulp and root canal. The periodontal ligament and pulp were removed mechanically, and dentin chips 2-3 mm in diameter were prepared by crushing the remaining dentin in a mortar and passing it through a 2-mm grid to maximize the size of the chips. The chips were cleaned from smaller dentin particles by soaking them in 1\% chlorhexidine for 5 min and then stored dry. For the comparative grafting intervention, a commercially available hydroxyapatite/biphasic tricalcium phosphate injectable putty bone-substitute material made of 60\% HA /40\% ß-TCP ratio (Maxresorb inject, Botiss Biomaterials, Berlin, Germany) was utilized and prepared per manufacturer's protocol. Dentin particles and B-TCP/HA putty were subsequently mixed together intraoperatively by the same operator for all interventions to ensure consistency of preparation and volume proportion and to create a moldable grafting mixture (Figure 2). The mixture was made using 0.5 cc of Maxresorb inject (60\% hydroxyapatite and 40\% $\beta$-tricalcium phosphate, Botiss Biomaterials, Berlin, Germany) with predetermined volume of dentin blocks obtained using the same dental scoop excavator. 
Figure 1: Alveolar cleft model.
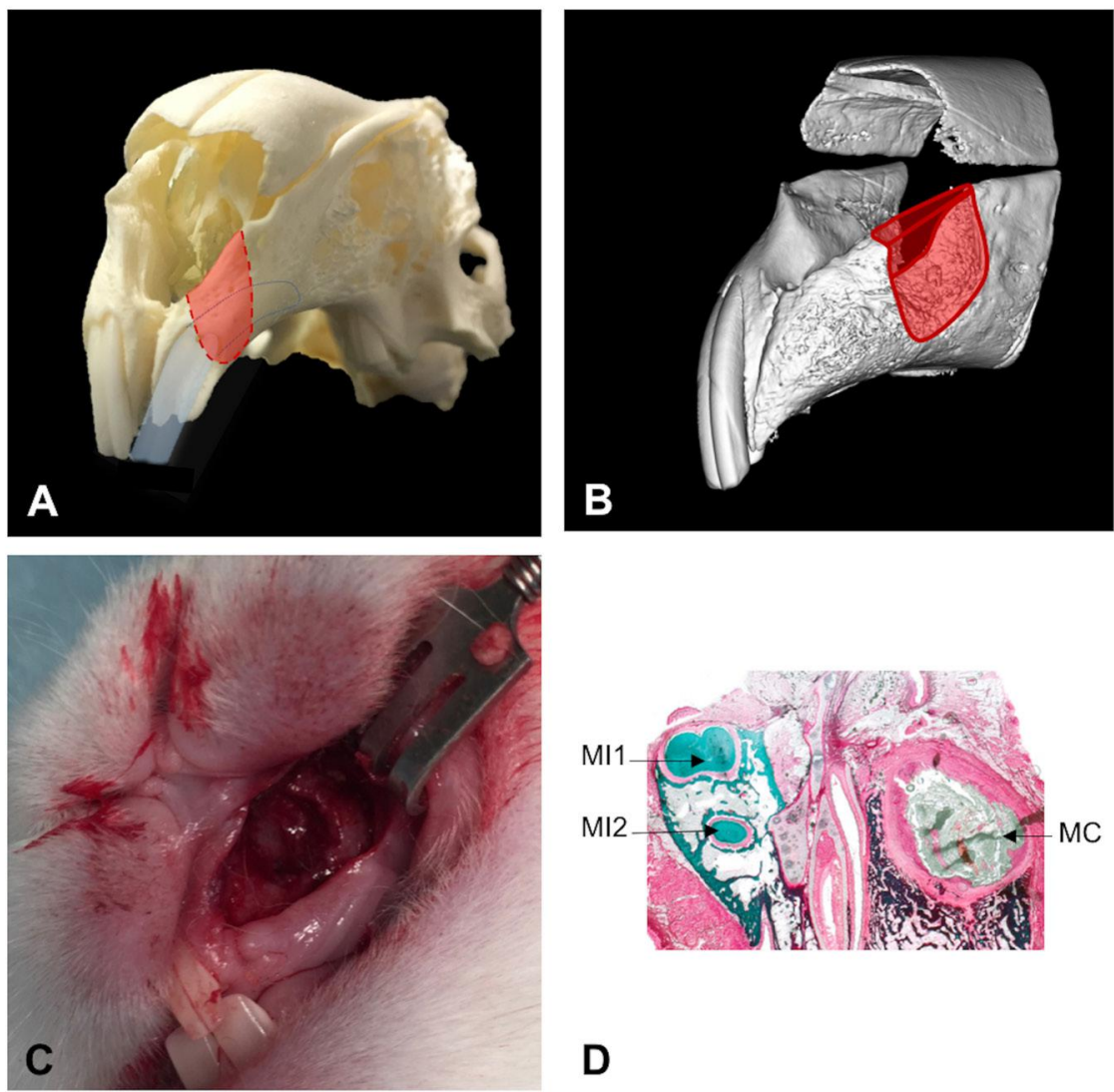

D

(A) Osteological representation of the created alveolar cleft extending to the nasal mucosa on a New Zealand White rabbit skull. (B) Micro-CT imaging of the created cleft 8 weeks after cleft creation surgery repesenting the three-dimensional morphology of the healed residual defect. (C) Surgical exposure of the healed alveolar cleft for insertion of the grafting intervention 8 weeks after cleft creation surgery. (D) Coronal histological representation of the healed alveolar cleft 8 weeks after cleft creation surgery with cleft area fully lined by mucosal tissue (MI1: first maxillary incisor; MI2: second maxillary incisor; MC: maxillary cleft).

\section{Preparation of surgical sites in Maxillary Alveolus}

The surgery was conducted under sterile conditions with the rabbits in a supine position. A buccal gingival flap was raised subperiosteally to expose the periodontal attachment of the central incisor tooth and the inferior nasal aperture as previously described in our model. ${ }^{50}$ Osteotomy was performed along the lateral curvature of the left central incisor using a rotary instrument to expose the root of the central incisor, then tooth was gently luxated laterally and extracted using extraction forceps. Further osteotomy was carried under protection of the nasal mucosa to remove the superior and inferior bony plates and 
create an oral-nasal defect with intact nasal mucosa. Bone wax (Ethicon Inc., Somerville, New Jersey, USA) then applied to the bony walls of the defect and the oral mucosa was approximated and sutured on the medial and lateral sides with 5 zeros resorbable Vicryl suture (Ethicon Inc., New Jersey, USA) leaving the central part of the wound open to create a pocket into the cleft defect, into which Surgicel oxidised cellulose (Johnson \& Johnson, New Brunswick, New Jersey, USA) was packed. The animals were allowed a period of 8 weeks for healing of the defect and the creation of maxillary alveolar defect. ${ }^{50}$

Figure 2: Graft preparation.
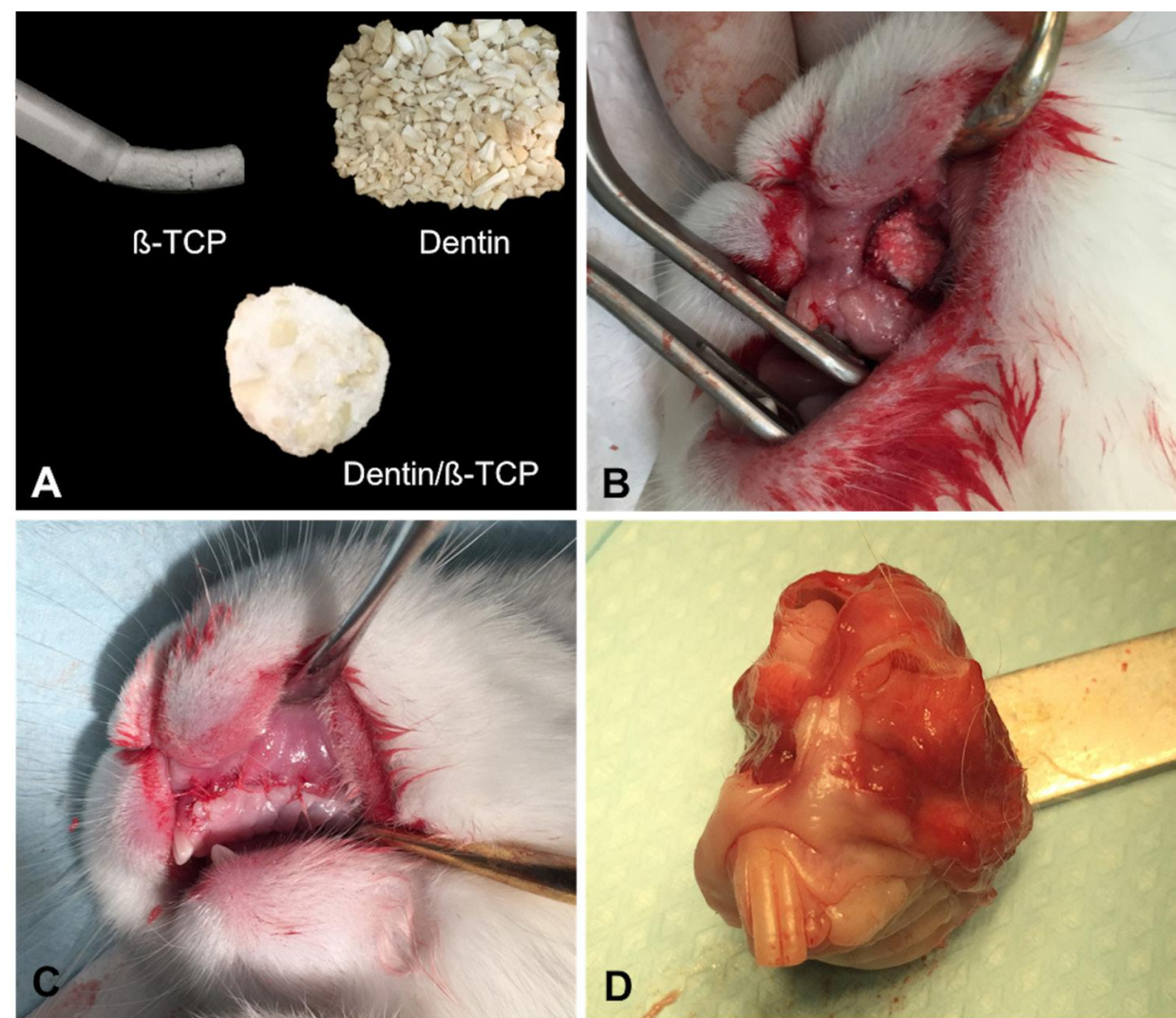

(A) Injectable ß-TCP/HA putty (Maxresorb inject, Botiss Biomaterials, Berlin, Germany), dentin blocks, and the in-situ formed composite dentin/ß-TCP mixture in preparation for grafting intervention. (B) Insertion of the grafting intervention in the alveolar cleft defect at 8 weeks after cleft creation surgery. (C) Tension-free closure of the oral mucosa over the grafted alveolar cleft defect. (D) Block sections of the maxilla specimens contained the repaired alveolar clefts 8 weeks after insertion of the grafting intervention. 
Exposure of the maxillary alveolus defects and insertion of the bone substitute materials:

The same surgical preparation and the strict protocol was carried during the second surgery to expose the alveolar defect in preparation of bone grafting. An incision in the non-keratinized mucosa is made in the alveolar defect area to separate the oral from the nasal mucosa. The bony defect was exposed and bony walls were lightly freshened with a round diamond bur. In 8 rabbits the defects were filled with composite xenogenic dentin with beta-tricalcium phosphate (intervention), whereas in 8 rabbits the defects were filled with beta-tricalcium phosphate alone (control) as seen in Figure 2. The grafting material was lightly placed into defect without compression. Primary wound closure was performed using 5 zeros resorbable Vicryl suture (Ethicon Inc., Somerville, New Jersey, USA). The rabbits were fed soft diet ad lib directly after the surgery. The rabbits were cared of accordingly per protocol and watched by veterinarian until the end of the study 3 months.

\section{Animal sacrifice and qualitative evaluation}

Animals were sacrificed at 8 weeks posoperatively with an intravenous lethal dose of T61 Euthanasia Solution (Embutramide, Mebezonium iodide, Tetracaine hydrochloride) after sedation with an intramuscular injection of Xylazine $\mathrm{HCl} 5 \mathrm{mg} / \mathrm{kg}$. Block sections of the maxilla specimens were obtained using oscillating saw maintaining the adjacent soft tissue and fixed in 10\% neutral-buffered Formalin (Figure 2). The specimens were grossly inspected for inflammation. Specimen were subsequently scanned with $\mu \mathrm{CT}$ before histological processing. Healing of the biomaterial-filled defects was compared radiographically and by histomorphometry. Micro-computed tomography $(\mu \mathrm{CT})$ was utilized to analyze the osteogenesis and healing patterns of the defects with different interventions. Quantitative analysis of bone mineral density (BMD) and bone volume fraction (\%) of the new bone formation ( $B V=$ bone volume/TV=tissue volume) was evaluated in the defects filled with each group.

\section{Acquisition of micro-CT scans, segmentation, and statistical analysis}

After animal sacrifice, the maxillas including the created alveolar defects with filling materials were harvested for ex vivo micro-CT imaging and histological analysis. To acquire the micro-CT images, maxillas were imaged ex vivo in $\mu \mathrm{CT}$ (Tomoscope 30s Duo, CT-Imaging, Erlangen, Germany) according to a protocol previously described by authors. ${ }^{51}$ After positioning in a multimodal holder, mandibles were scanned with the $\mu \mathrm{CT}$. A dual energy scan (HQD-6565-360-29) which acquires 720 projections with $1032 \times 1012$ pixels with scanning time 90 sec per subscan was used. To cover one maxilla, two subscans were acquired. Both tubes of the dual source $\mu \mathrm{CT}$ were operated with voltage of $65 \mathrm{kV}$ and current of 1 $\mathrm{mA}$. The $\mu \mathrm{CT}$ data was reconstructed at an isotropic voxel size of $35 \mu \mathrm{m}$ using a Feldkamp

type algorithm and a smooth kernel. For analysis, the $\mu \mathrm{CT}$ data was down-sampled using binning to a voxel size of $70 \mu \mathrm{m}$. To determine the bone mineral density score and the 
new formed bone volume the $70 \mu \mathrm{m} \mu \mathrm{CT}$ file was analyzed with the Imalytics Preclinical software. ${ }^{52}$ To segment the alveolar cleft, scribbles were drawn slice-wise to delineate the boundaries of the fillings and newly formed bones. A threshold over $600 \mathrm{HU}$ was applied to segment the bones inside the area. Relative bone volume and mean intensity (as bone density score) inside the segmented class were determined as volumetric ratio. As an added measure to reduce bias in the measurement readings of the defect size on $\mu C T$ images, measurements of defect size determination were scored independently by two imaging specialists and confirmed by the operator. The statistical data was analyzed using GraphPad Prism (Version 6.0 for Windows, GraphPad Software, La Jolla, California, USA)). To compare the measurements between TCP and dentin, unpaired t-test was performed in combination with a Tukey post-test. A p-value below 0.05 was considered to represent statistical significance.

\section{Histologic preparation histomorphometry, and statistical analysis}

After $\mu \mathrm{CT}$ image acquisition, the specimens were dehydrated using ascending ethanol gradient (50-100\%) prior to embedding in Methylmethacrylate resin (Technovit 9100, Heraeus Kulzer GmbH, Frankfurt, Germany). Coronal sections of the embedded undecalcified specimens were obtained at a thickness of about $200 \mu \mathrm{m}$ using the EXAKT cutting unit (EXAKT Technologies Inc., Oklahoma City, Oklahoma, USA), then thinned and polished manually to a final thickness of about 50-70 $\mu \mathrm{m}$. Final specimens were stained with Masson's Trichrome (MTC) and Toluidine blue (TB) according to protocol, and were analyzed using light microscopy. Three slides for each defect was obtained in the coronal section through the center grafted defect after visual inspection of the embedded specimens. The middle slice was selected for analysis in all the cases. Histomorphometric evaluation for performed with ImageJ software (Image 1.51 p, National Institute of Health, Bethesda, Maryland, USA) by manually measuring the compartments of new bone area, and remaining grafting materials. Mean percentage of bone formation per total defect volume $(\%=$ new bone area / total defect area) and standard deviation (SD) were used for each group. Statistical analysis conducted using GraphPad Prism (Version 6.0 for Windows, GraphPad Software, La Jolla, California, USA) using unpaired t-test was performed in combination with a Tukey post-test. A p-value below 0.05 was considered to represent statistical significance. 


\section{RESULTS}

\section{Surgical procedure and experimental observations}

All rabbits survived the surgical procedure. In a few cases an extra injection of Ketamine was required. Handling of the putty B-TCP/HA during surgery was very effective and facilitated the in-situ mixing with dentin chips and insertion of the composite graft in the alveolar defect without the need of any additional handling, such as wetting or drying. The graft showed acceptable dimensional stability and retained in the defect adequately. Intraoperative and postoperative bleeding was minimal and did not result in any clinical issues. The animals were active and behaved adequately immediately after the surgery. They all started eating already during the first day after surgery. The animals were fed soft pellets ad libitum throughout the study and gained weight. All the rabbits survived during the 8 weeks postoperatively until day of the sacrifice.

Figure 3: $\mu C T$ Imaging.

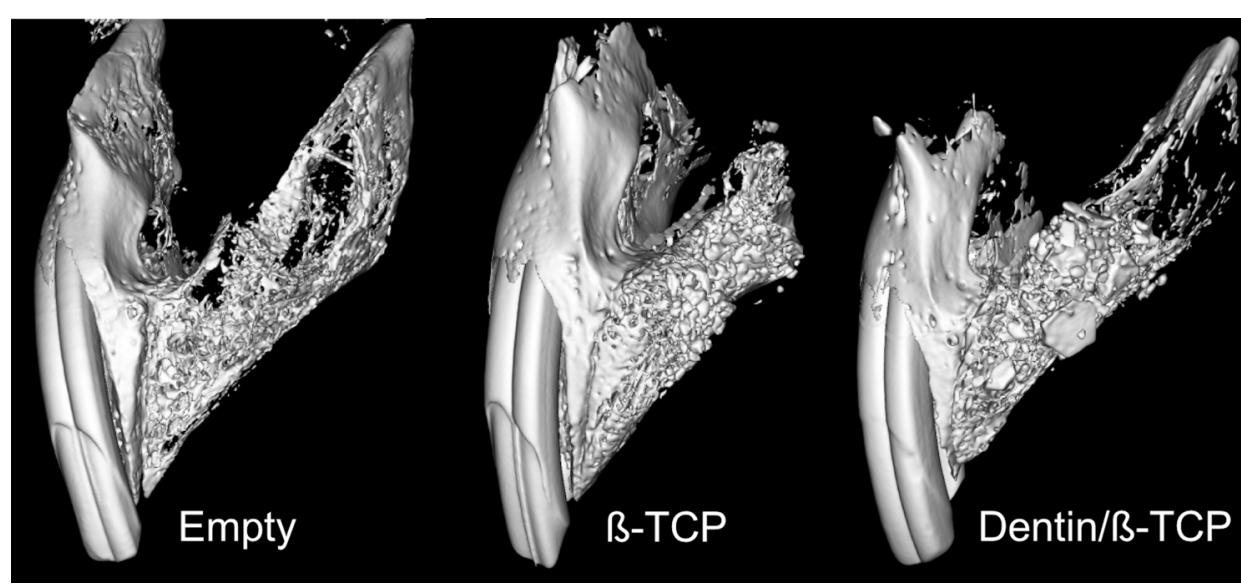

$\mu \mathrm{CT}$ imaging reconstruction of the created alveolar clefts 8 weeks after cleft creation surgery, and 8 weeks after insertion of the grafting intervention with ß-TCP and Dentin/ß-TCP composite.

HCT imaging of the filled alveolar defects:

The results from $\mu C T$ imaging showed that in both defect groups there were bridging of bone and filling of the defects at the 8-week time (Figure 3). Statistical analysis of the imaging parameters (Table 1) showed that there was significance difference in the Hounsfield unit (HU) between clefts filled with Dentin/B-TCP (3455 \pm 150.3 ) versus defects filled with B-TCP (3421 \pm 103.2$)$. Furthermore, there was a statistically significant higher amount of percentage bone volume fraction (BV/TV)noted in the composite Dentin/B-TCP filled defects by $\mu \mathrm{CT}(78.46 \pm 4.16)$ compared to ß-TCP group (60.39 \pm 4.5$)$, likely representing 
higher bone formation activity (Figure 4). The Bone Mineral Density (BMD) comparison correlated also with these radiographic findings with significantly higher values in the composite Dentin/ß-TCP filled defects (1185 \pm 34.68) when compared to the ß-TCP filled defects (1028 \pm 41.87) (Figure 4).

Table 1. Results of the $\mu \mathrm{CT}$ measurements

\begin{tabular}{lccccc}
\hline & $\begin{array}{c}\text { Nr. of } \\
\text { Clefts }\end{array}$ & Defect Size $\mathbf{m m}^{3}$ & $\begin{array}{c}\text { Hounsfield Unit } \\
(\mathbf{H U})\end{array}$ & $\begin{array}{c}\text { \% Bone Volume } \\
\text { Fraction (BV/TV) }\end{array}$ & $\begin{array}{c}\text { Bone Mineral } \\
\text { Density (BMD) }\end{array}$ \\
\hline B-TCP & 8 & $119.84 \pm 32.88$ & $3421 \pm 103.2$ & $60.39 \pm 4.5$ & $1028 \pm 41.87$ \\
Dentin/B-TCP & 8 & $151.38 \pm 29.53$ & $3455 \pm 150.3$ & $78.46 \pm 4.16$ & $1185 \pm 34.68$ \\
Mean Difference & & $-31.54 \pm 15.62$ & $33.95 \pm 182.3$ & $18.07 \pm 6.13$ & $156.8 \pm 53.9$ \\
P-Value & 0.063 & & 0.0121 * & 0.0122 * \\
95\% Cl of MD & & -65.05 to 1.97 & & 4.726 to 31.42 & 40.4 to 273.30 \\
\hline
\end{tabular}

Mean Hounsfield unit (HU), \% Bone Volume Fraction (BV/TV), and Bone Mineral Density (BMD) in defect groups, (means \pm standard deviations), Cl: Confidence Interval, MD=mean difference.

* statistically significant.

\section{Histomorphometric analysis}

Histological analysis after 8 weeks showed bone healing pattern which correlated well with the $\mu \mathrm{CT}$ findings. Both ß-TCP and Dentin/ß-TCP filled cavities showed enhanced new bone formation. Histomorphometric analysis showed higher percentage of bone volume fraction ( $p<0.05)$ in Dentin/ß-TCP group $(55,06 \% \pm 7,20)$ compared to $ß$-TCP group $(30,08 \%$ $\pm 9,08$ ) (Table 2, Figure 5, Figure 6). Percentage residual graft was also significantly higher $(p<0.001)$ in Dentin/ß-TCP groups $(47,44 \% \pm 6,72)$ with grossly visible dentin chips compared to the ß-TCP group $(22,46 \% \pm 2,80)$, which also showed a lower graft area on gross examination of the slides (Figure 5). Areas of fused dentin with bone (ankylosis) was noted, and resorption cavities in the dentin were also noted (Figure 6).

Table 2. Results of histomorphometry measurements

\begin{tabular}{lccc}
\hline & N & \% Bone Formation & \% Residual Graft \\
\hline B-TCP & 8 & $30,08 \pm 9,08$ & $22,46 \pm 2,80$ \\
Dentin/B-TCP & 8 & $55,06 \pm 7,20$ & $47,44 \pm 6,72$ \\
Mean Difference & $-24.98 \pm 3.71$ & $-28.17 \pm 2.92$ \\
P-Value & $0.0114 *$ & 0.0017 * \\
95\% Cl of MD & & -32.93 to -17.02 & -37.24 to -20.73 \\
\hline
\end{tabular}

$\%$ Bone Formation=NBA/TDA $\times 100$ and \% Residual Graft=RGA/TDA in defect groups, (means \pm standard deviations)

NBA: new bone area; TDA: total defect area; RGA: residual graft area); Cl: Confidence Interval; MD, mean difference.

* statistically significant. 


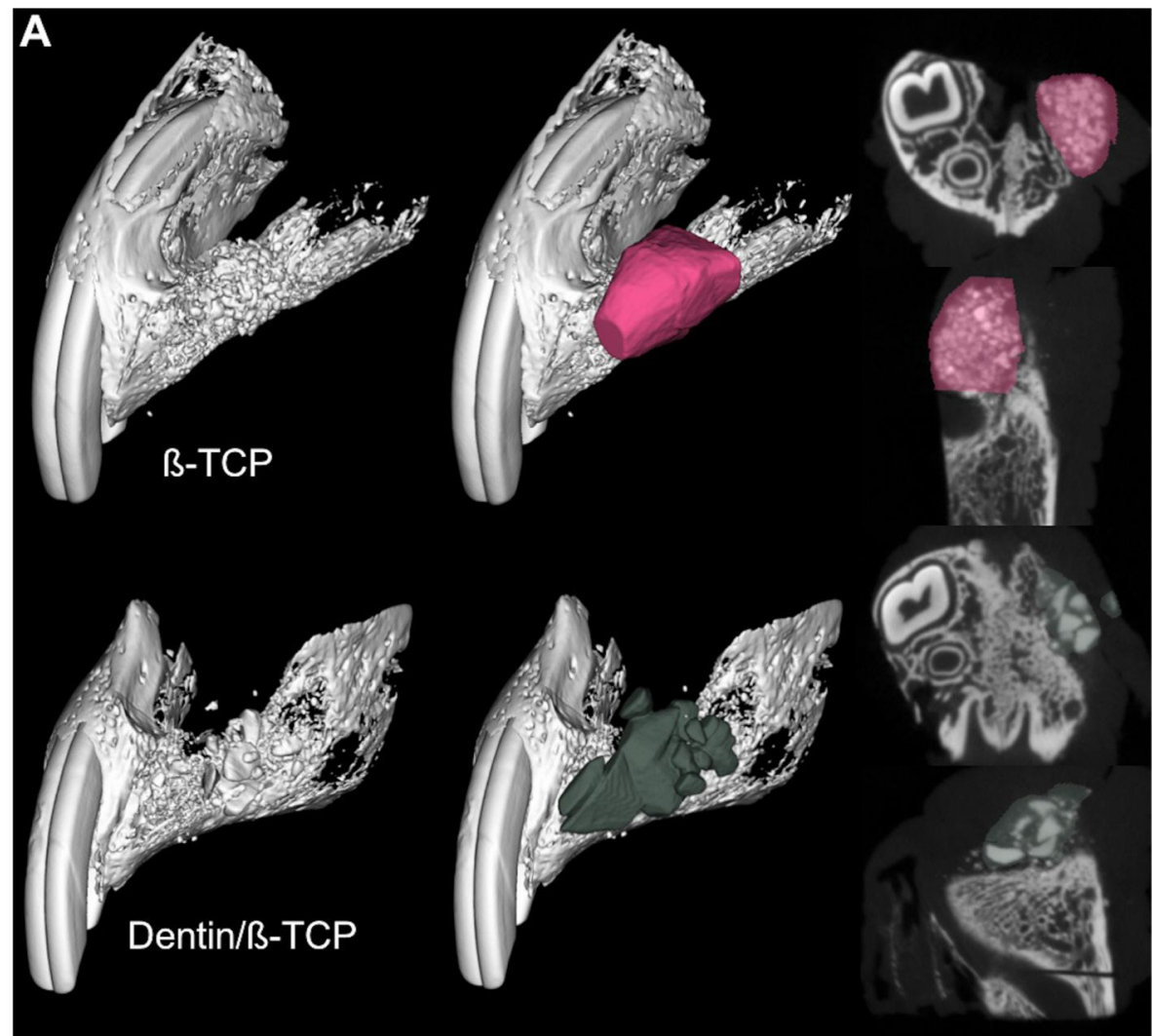

B

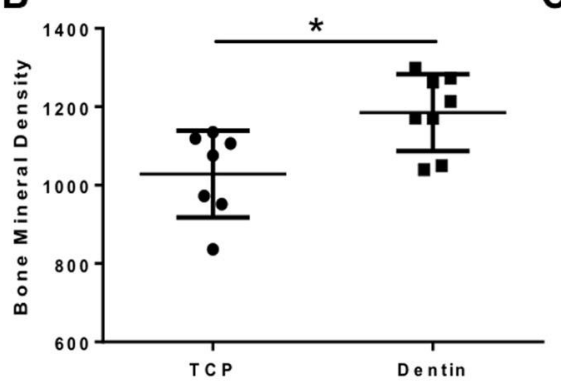

C

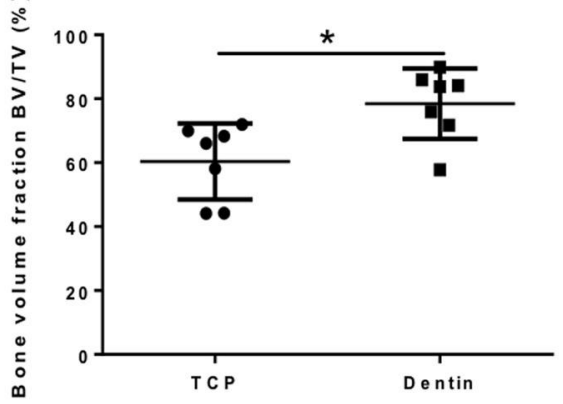

(A) Healed alveolar cleft 8 weeks after insertion of the grafting intervention with ß-TCP and Dentin/ß-TCP, color outlining the residual volume of the grafted area. (B) Quantitative analysis of bone mineral density (BMD) of defect areas filled with B-TCP or Dentin/ß-TCP. (C) Bone volume fraction (\%) of the bone volume fraction (BV=bone volume/TV=tissue volume) in the defect area of each group. (* indicates $p<0.05$ ) 
Figure 5: Histological representation of the repaired alveolar clefts.

A

B-TCP

Masson's Trichrome

Toluidine blue

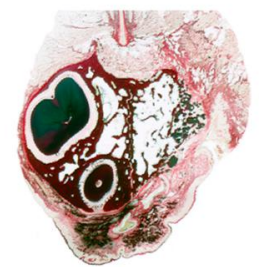

B
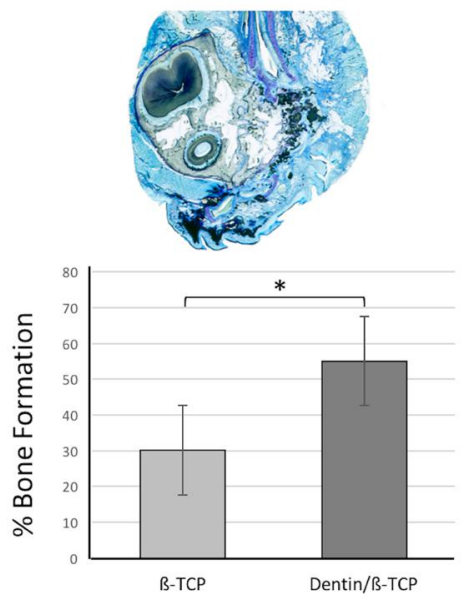

Dentin/ß-TCP
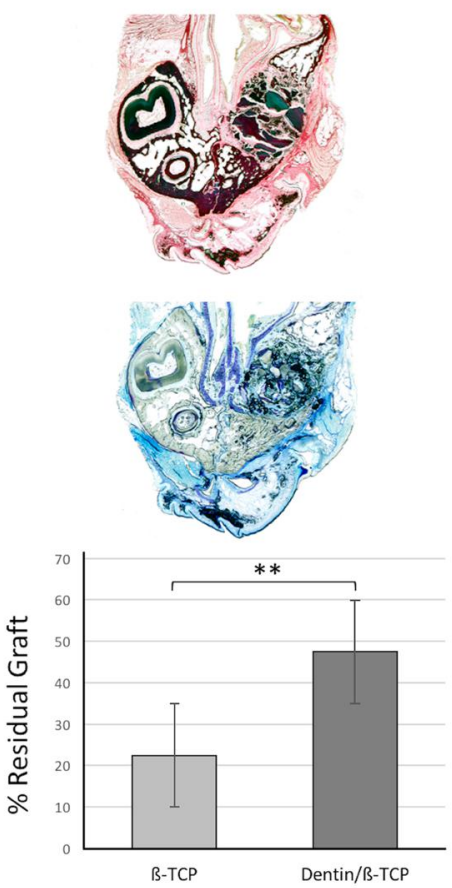

(A) Coronal sections of the repaired alveolar clefts 8 weeks after insertion of the grafting intervention stained with Masson's Trichrome and Toluidine Blue. (B) Quantitative analysis of percentage bone formation and percentage residual grafts of defect areas filled with B-TCP or Dentin/ß-TCP. (* indicates $p<0.05$, ** $p<0.01$ ) 
Figure 6: Histological representation of the repaired alveolar clefts.
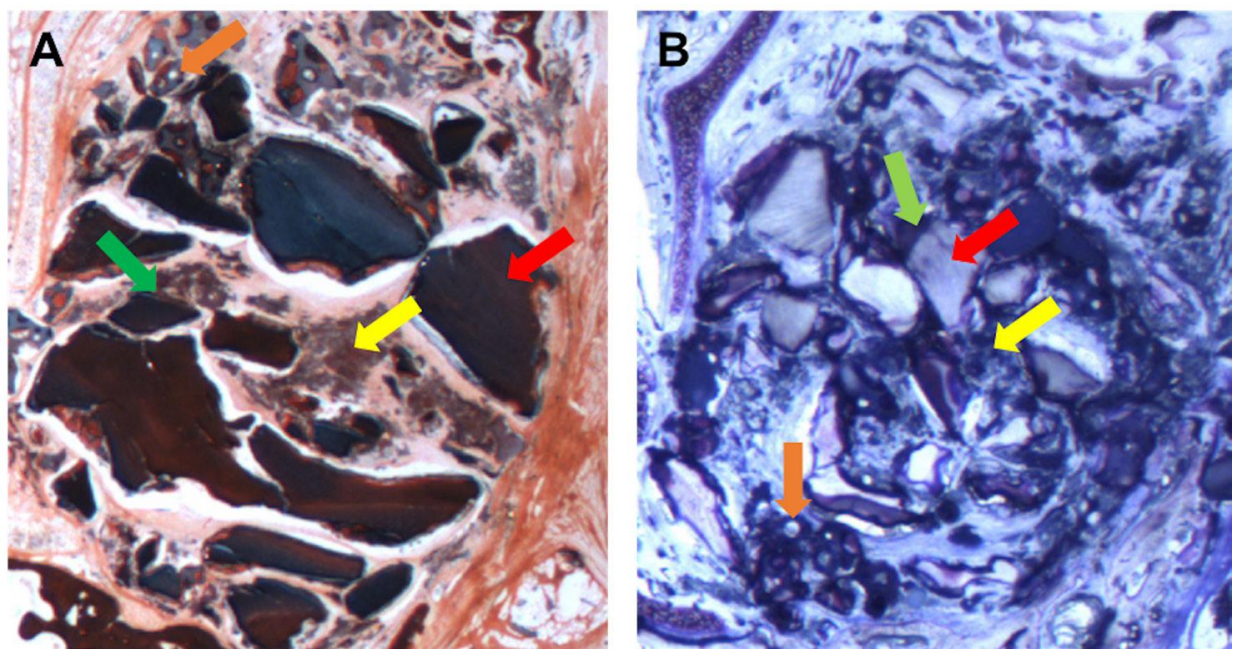

Histological sections of the repaired alveolar clefts 8 weeks after insertion of the grafting intervention stained with Masson's Trichrome (A) and Toluidine Blue (B). Red arrow: residual dentin; yellow arrow: newly formed bone; fused dentin with bone; orange arrow: resorption lacunae associated with B-TCP. 


\section{DISCUSSION}

Maxillary alveolar defects are anatomically unique with regard to their overlying soft tissues; the oral mucosa inside the mouth and the nasal mucosa as the nasal floor lining, each with distinct bio-environment. This study demonstrated the feasibility of establishing a reliable skeletal defect in rabbits prior to the later grafting of the defect in a two stage surgery to study alveolar cleft repair healing pattern using various novel biomaterials in three-dimensional form similar in morphology to human patients. ${ }^{50}$ Many previous studies in the literature have only filled a created a bone defect in a one stage surgery 45, 47, 53-55, which is not in accordance with closing a cleft clinically where oral and nasal epithelium is lining the defect.

A suitable bone substitute grafting material for alveolar cleft repair should have osteoinductive and osteoconductive properties, to promote new bone formation, in addition to reliably resorbable scaffold that is subsequently replaced with functionally viable bone that would allow teeth movement into the defect and eruption of teeth. 2, 3, 6, 9-12,56 The major issue encountered in alveolar cleft repair is the inherent resorption rate associated with autogenous bone grafts, therefore, several studies have focused on attempting to develop new bone substitute materials for bone regeneration that would be less susceptible to bone resorption through several methods including cell therapy with enhanced tissue-engineered scaffolds using mesenchymal stem cells (MSC) or osteoblasts (OB), application of growth factors such as bone morphogenic proteins (BMP), platelet rich plasma (PRP), platelet derived growth factors (PDGF), or various alloplastic biocomposites including calcium phosphate (TCP), hydroxyapatite (HA), or bioglass. 4, 8, 11, 12, 56, 57

Calcium phosphate ceramics are widely utilized in clinical practice as bone substitute materials and contain various forms of alloplastic biomaterials, mostly as calcium sulfate, tricalcium phosphate and the recently introduced biphasic TCP (ß-TCP). ${ }^{58,59}$ ß-TCP is available as bone grafting materials in powder granules, blocks, and as injectable paste with nano-micro hydroxyapatite granules to create a non-hardening a moldable mixture, like Maxresorb (Botiss Biomaterials, Berlin, Germany). Calcium phosphate ceramics are often subject to prolong healing time given their lack of osteoindutive properties and their graft volume maintenance depends on the level of resorbability of these alloplastic materials and their replacement rate by bone. ${ }^{59-61}$ For modification of the $\beta$-TCP resorption properties, a balanced ratio of HA and $\beta$-TCP is created to achieve a suitable resorption rate to allow bone formation by replacement, with most studies reporting optimal clinical HA/ $\beta$-TCP ratio between 65:35 and 55:45. 58,62-64 
Since HA particles are less susceptible to resorption and acts as structural scaffold for ingrowing osteogenesis and angiogenesis while the dissolution of $\beta$-TCP during grafting procedures yields Ca and $\mathrm{PO} 4$ ions, which have also been suggested to stimulate bone formation. 58,59, 65 De Ruiter et. al. has compared bone healing with B-TCP and autogenous bone in alveolar cleft repair in a goat model and reported that B-TCP resulted in bone healing similar to iliac crest bone. ${ }^{2}$ They reported increased histologic bone formation area in B-TCP group (22.90\%, SD 5.62) compared to autogenous iliac crest (20.87\%; 5.40), however, no statistical difference was found between the results. ${ }^{2}$ A later study by Janssen et. al. evaluated microstructured B-TCP granules, embedded in a carboxymethyl cellulose in glycerol (CMCG) putty as alternative grafting material in comparison to autogenous iliac crest bone for alveolar cleft repair in a goat bilateral alveolar cleft model. ${ }^{3}$ They reported an improved surgical handling of the ß-TCP material during alveolar cleft repair with volumetric $\mu C T$ and histomorphometric analyses demonstrating integration of both autogenous and B-TCP/CMCG with no significant differences between both groups in percentages of bone formation on the histological sections or reconstructed bone volume with $\mu \mathrm{CT} .{ }^{3}$

Previous studies on teeth replantation have demonstrated that delayed replantation of teeth, where the periodontal ligament has become injured and necrotic e.g. by extensive drying, the dentin will fuse with bone and undergo ankylosis. ${ }^{21,29-31,66,67}$ The ankylosed teeth, which made mostly of dentin, undergo gradual resorption process and later go through osseous replacement in a process called replacement resorption where the dentin is replaced by bone. . $1,23-25,29,30,66$ This has prompt further investigations into dentin as bone grafting materials. Additional studies have utilized this clinical phenomenon to preserve alveolar ridge height and width through decoronation of tooth or delayed replantation of teeth into the alveolar process to be slowly replaced by newly formed bone, thus maintaining the volume of alveolar process and preventing its resorption. ${ }^{31,66,68,69}$

Further experimental studies in rabbits demonstrated the ability of human xenogenic dentin blocks grafts to integrate into native bone and then slowly replaced by new bone with minimal inflammatory reaction. ${ }^{24,25}$ They reported integration of the dentin grafts with bone in $86 \%$ of the dentin surface after 3 months and $98 \%$ after 6 months. Interesting was their findings of showing continuous resorption of dentin with bony remodelling after 6 months with osseous replacement in the resorption cavities in a gradual process with subsequent replacement of dentin with new bone. Several studies demonstrated the lack of inflammation during healing which can be suggestive of the low level of immunogenicity with dentin. ${ }^{21,24,25}$ indicating that this is mere a remodelling resorption process than infective related. ${ }^{29,66}$ 
Additional studies have shown that human dentin possesses osteoinductive properties, likely explained by its inherent reservoir of bone morphogenic protein (BMP) and its function as a carrier of these growth factors. ${ }^{21,32,70,71}$ The osteoinductivity potential of dentin was further investigated in a study by study by Al-Asfour et al, they demonstrated that xenogenic non-demineralized dentin can promote new bone formation when placed in the marrow space of rabbits tibia. ${ }^{21}$ In their study, more bone formation was seen on the dentin when the graft was placed close proximity to native bone, suggesting a bigger role of osteoconductive properties of dentin during graft healing in comparison to the osteoinductive properties. ${ }^{21}$ Additional work by the same research group demonstrated minimal bone formation when dentin was implanted in non-osteogenic environment suggesting a decrease role ostoinductivity non-demineralized dentin. ${ }^{23}$

Mordenfeld et. al. investigated the effect of demineralization of xenogenic porcine dentin on bone formation in an osteoconductive environment in a rat model and noted a higher rate of resorption of the dentin grafts with increasing level of graft demineralization. ${ }^{33}$ Their results also demonstrated a significant increase in bone formation with increasing the degree of demineralization of the dentin, as long as minimal inflammatory process was present around the dentin graft. This suggest that bone regeneration and healing of the dentin graft was closely related to the inflammatory process during healing phase, and that incorporation of dentin with native bone was seen when inflammation is absent, with subsequent gradual resorption and replacement with bone. 21, 24, 25, 33, 61 Mordenfeld et. al. study also demonstrated that grafting dentin resulted in fibrous encapsulation and compromised graft healing when dentin blocks were placed loosely beneath the periosteum of rat skull, likely as a result of the micromovements caused by non-fixation of the dentin graft during bone healing phase. On the other hand, superior graft healing and incorporation into bone was seen when dentin blocks were inserted into a tighter pocket, which restricted the mobility of the graft. ${ }^{33}$ These results are consistent with findings from other animal studies which demonstrated that dentin blocks undergo resorption when implanted in a non-osteogenic environment, such as in the muscle or the subcutaneous tissue, due to mobility of the graft leading to fibrous tissue reaction and inability to induce bone formation. ${ }^{21,23,34,72}$ For this reason, the stability of the dentin graft, local tissue environment around the graft, and the susceptibility to inflammatory process seems to have an essential role in healing of the dentin grafts and its incorporation into bone. ${ }^{21,23-25,31,33,34}$ Further comparative study of bone regeneration pattern in rabbit's tibia between autogenous onlay bone blocks and xenogenic deminerlaized dentin onaly blocks were conducted by Al-Asfour et. al, and demonstrated notable new bone formation through replacement of the integrated dentin at the native bone/dentin graft interphase with similar resorption rate and pattern of the dentin blocks to the autogenous bone over 12 weeks period. ${ }^{22}$ By constructing a composite putty ß-TCP/HA and dentin blocks mixture, we aimed at improv- 
ing the handling properties of the dentin blocks during graft insertion into alveolar clefts and restricting dentin grafts mobility, hence achieving better graft stability in the defect to evaluate of the regenerative potential of dentin/ ß-TCP graft on osteogenesis.

Our results show that alveolar clefts defects filled with composite Dentin/ß-TCP showed the highest number of bone volume fraction (BV/TV) at (78.46\% \pm 4.16$)$, and bone mineral density (BMD) $(1185 \pm 34.68)$ when compared to the alveolar defects filled with B-TCP alone. (Table 1) These differences were statistically different (alpha <0.05) suggesting that the composite Dentin and B-TCP/HA mixture has successfully healed with the native bone. The higher \% Bone Volume Fraction and BMD can be likely attributed to the residual dentin grafts, which show a slower rate of resorption and may possess inherent osteoinductive properties. This correlated well with previous finding reported by Al-Asfour, Andersson, and Mordenfeld groups. ${ }^{21-25,33}$ Our histological findings correlated well with our radiographic findings with composite Dentin/ß-TCP demonstrating statistically higher (alpha $<0.05)$ bone volume fraction $(55,06 \% \pm 7,20)$ compared to ß-TCP group $(30,08 \% \pm 9,08)$, suggesting superior overall bone volume formation in the composite group. Of note was the significantly higher percentage residual graft in the in addition to higher percentage of residual grafts in the Dentin/ß-TCP group $(47,44 \% \pm 6,72)$ compared to the $ß$-TCP group $(22,46 \% \pm 2,80)$ after 8 weeks healing period (Table 2$)$, which is consistent with previous studies reporting slower resorption rate of dentin grafts which leads to better graft volume maintenance. ${ }^{21,22,24,25,33,34,61}$ In our study, the ß-TCP/HA mixture was non-hardening, and served the purpose of stabilized the dentinal blocks to allow osteogenesis and expression of any osteoinductive properties.

The results of this study demonstrated a reliable alveolar cleft animal model of testing of novel bone substritute materials after successull grafting and bone formation, and that alveolar cleft defects repair with Dentin/ß-TCP achieved higher graft residual volume and bone volume fraction when evaluated by $\mu \mathrm{CT}$ and histomorphometry. 


\title{
CONCLUSIONS
}

Grafting of alveolar cleft defects in rabbits with composite xenogenic dentin with beta-tricalcium phosphate achieved supererior bone volume fraction and residual graft volume to clefts repaired with beta-tricalcium phosphate alone when evaluated radiographically and histologically.

\section{ABBREVATIONS:}

\author{
BMD: Bone mineral density \\ B-TCP: Beta-Tricalcium Phosphate. \\ BV/TV: \% Bone Volume Fraction \\ HA: Hydroxyapetite \\ HU: Hounsfield unit \\ $\mu \mathrm{CT}$ : Micro-CT \\ NZW: New Zealand White rabbit. \\ SD: Standard deviation
}

\section{AUTHORS' CONTRIBUTIONS:}

$M K, B L, A B$ and $P K$ designed the study. MK, LA and AA prepared the grafting material and defined the protocol. MK, LA, and BL were responsible for the in-vivo surgery and performing the procedure. FG and SR were responsible for imaging acquisition and analysis. $M K, A B, B L$ prepared the manuscript. LA, FH, PK, AA and LA were responsible for revising the manuscript critically for important intellectual content. All Authors read and approved the final manuscript.

\section{ACKNOWLEDGEMENTS:}

The authors are grateful to Dr. Severino Gabato for assisting in the in-vivo surgery and taking excellent care of the animals. The authors would also like to thank Ms. Nicole Bataille for her generous help with preparing the histology slide and light microscopy. 


\section{COMPETING INTERESTS:}

The authors report no conflicts of interest related to this study.

\section{AVAILABILITY OF DATA AND MATERIAL:}

The data/material analysed during the current study available from the curresponding author on request.

\section{CONSENT FOR PUBLICATION:}

Not applicable

\section{ETHICS APPROVAL AND CONSENT TO PARTICIPATE:}

All animals were handled in compliance with European Communities Council Directive of 24 November 1986 (86/609/EEC) for the care of laboratory animals in experimental procedures and ethical guidelines for animal research. ${ }^{73}$ All methods were carried out in accordance with the approved guidelines and regulations of the HSC Ethical Committee for the use of Laboratory Animals in Teaching and in Research, Kuwait University.

\section{FUNDING:}

This study was supported by the resources of Health Sciences Centre, Kuwait University and RWTH Aachen University, Germany. 


\section{REFERENCES}

1. Rychlik D, Wójcicki P, Koźlik M. Osteoplasty of the alveolar cleft defect. Adv Clin Exp Med2012. p. 255-62.

2. de Ruiter A, Meijer G, Dormaar T, Janssen N, van der Bilt A, Slootweg P, et al. $\beta$-TCP versus autologous bone for repair of alveolar clefts in a goat model. Cleft Palate Craniofac J: Allen Press Publishing Services; 2011. p. 654-62.

3. Janssen NG, de Ruiter AP, van Hout WM, van Miegem V, Gawlitta D, Groot FB-d, et al. Microstructured $\beta$-Tricalcium Phosphate Putty Versus Autologous Bone for Repair of Alveolar Clefts in a Goat Model. The Cleft Palate-Craniofacial Journal. 2016.

4. Yuanzheng C, Yan G, Ting L, Yanjie F, Peng W, Nan B. Enhancement of the repair of dog alveolar cleft by an autologous iliac bone, bone marrow-derived mesenchymal stem cell, and platelet-rich fibrin mixture. Plast Reconstr Surg2015. p. 1405-12.

5. Seifeldin SA. Is alveolar cleft reconstruction still controversial? (Review of literature). Saudi Dent J2016. p. 3-11.

6. Chung VH, Chen AY, Jeng LB, Kwan CC, Cheng SH, Chang SC. Engineered autologous bone marrow mesenchymal stem cells: alternative to cleft alveolar bone graft surgery. The Journal of craniofacial surgery. 2012;23:1558-63.

7. Ma'amon AR, Telfah H. Secondary alveolar bone grafting: the dilemma of donor site selection and morbidity. British Journal of Oral and Maxillofacial Surgery. 2008;46:665-70.

8. Jensen AT, Jensen SS, Worsaae N. Complications related to bone augmentation procedures of localized defects in the alveolar ridge. A retrospective clinical study. Oral and maxillofacial surgery. 2016;20:115-22.

9. Gładysz D, Hozyasz KK. Stem cell regenerative therapy in alveolar cleft reconstruction. Arch Oral Biol2015. p. 1517-32.

10. Kawata T, Kohno S, Fujita T, Sugiyama H, Tokimasa C, Kaku M, et al. New biomaterials and methods for craniofacial bone defect: chondroid bone grafts in maxillary alveolar clefts. Journal of craniofacial genetics and developmental biology. 2000;20:49-52.

11. Janssen NG, Weijs WLJ, Koole R, Rosenberg AJWP, Meijer GJ. Tissue engineering strategies for alveolar cleft reconstruction: a systematic review of the literature. Clin Oral Investig: Springer Berlin Heidelberg; 2014. p. 219-26.

12. Khojasteh A, Kheiri L, Motamedian SR, Nadjmi N. Regenerative medicine in the treatment of alveolar cleft defect: A systematic review of the literature. J Craniomaxillofac Surg2015. p. 1608-13.

13. Su-Gwan K, Hak-Kyun K, Sung-Chul L. Combined implantation of particulate dentine, plaster of Paris, and a bone xenograft (Bio-Oss $®$ ) for bone regeneration in rats. Journal of Cranio-Maxillofacial Surgery. 2001;29:282-8.

14. Clokie CM, Moghadam H, Jackson MT, Sandor GK. Closure of critical sized defects with allogenic and alloplastic bone substitutes. Journal of Craniofacial Surgery. 2002;13:111-21.

15. Haddad AJ, Peel SA, Clokie CM, Sándor GK. Closure of rabbit calvarial critical-sized defects using protective composite allogeneic and alloplastic bone substitutes. Journal of Craniofacial Surgery. 2006;17:926-34.

16. Lappalainen O-P, Karhula S, Haapea M, Kyllönen L, Haimi S, Miettinen S, et al. Bone healing in rabbit calvarial critical-sized defects filled with stem cells and growth factors combined with granular or solid scaffolds. Child's Nervous System. 2016;32:681-8.

17. Lappalainen O-P, Karhula SS, Haapea M, Kauppinen S, Finnilä M, Saarakkala S, et al. Micro-CT Analysis of Bone Healing in Rabbit Calvarial Critical-Sized Defects with Solid Bioactive Glass, Tricalcium Phosphate Granules or Autogenous Bone. Journal of Oral \& Maxillofacial Research. 2016;7.

18. Saikia K, Bhattacharya T, Bhuyan S, Talukdar D, Saikia S, Jitesh P. Calcium phosphate ceramics as bone graft substitutes in filling bone tumor defects. Indian journal of orthopaedics. 2008;42:169. 
19. Zhou AJ-J, Peel SA, Clokie CM. An evaluation of hydroxyapatite and biphasic calcium phosphate in combination with Pluronic F127 and BMP on bone repair. Journal of Craniofacial Surgery. 2007;18:1264-75.

20. Bittermann GKP, Janssen NG, van Leeuwen M, van Es RJ. One-Year Volume Stability of Human Facial Defects Filled With a $\beta$-Tricalcium Phosphate-Hydroxyl Apatite Mixture (Atlantik). Journal of Craniofacial Surgery. 2014;25:372-4.

21. Al-Asfour A, Andersson L, Kamal M, Joseph B. New bone formation around xenogenic dentin grafts to rabbit tibia marrow. Dental traumatology : official publication of International Association for Dental Traumatology. 2013;29:455-60.

22. Al-Asfour A, Farzad P, Al-Musawi A, Dahlin C, Andersson L. Demineralized Xenogenic Dentin and Autogenous Bone as Onlay Grafts to Rabbit Tibia. Implant dentistry. 2017;26:232-7.

23. Al-Asfour A, Farzad P, Andersson L, Joseph B, Dahlin C. Host tissue reactions of non-demineralized autogenic and xenogenic dentin blocks implanted in a non-osteogenic environment. An experimental study in rabbits. Dental traumatology : official publication of International Association for Dental Traumatology. 2014;30:198203.

24. Andersson L. Dentin xenografts to experimental bone defects in rabbit tibia are ankylosed and undergo osseous replacement. Dental traumatology : official publication of International Association for Dental Traumatology. 2010;26:398-402.

25. Andersson L, Ramzi A, Joseph B. Studies on dentin grafts to bone defects in rabbit tibia and mandible; development of an experimental model. Dental traumatology : official publication of International Association for Dental Traumatology. 2009;25:78-83.

26. Fugazzotto P, De Paoli S, Benfenati S. The use of allogenic freeze-dried dentin in the repair of periodontal osseous defects in humans. Quintessence international (Berlin, Germany: 1985). 1986;17:461.

27. Gomes MF, dos Anjos MJ, Nogueira TO, Guimaraes SA. Histologic evaluation of the osteoinductive property of autogenous demineralized dentin matrix on surgical bone defects in rabbit skulls using human amniotic membrane for guided bone regeneration. The International journal of oral \& maxillofacial implants. 2001;16:563-71.

28. Moharamzadeh K, Freeman C, Blackwood K. Processed bovine dentine as a bone substitute. British Journal of Oral and Maxillofacial Surgery. 2008;46:110-3.

29. Andersson L. Dentoalveolar ankylosis and associated root resorption in replanted teeth. Experimental and clinical studies in monkeys and man. Swedish dental journal Supplement. 1988;56:1-75.

30. Andersson L, Blomlöf L, Lindskog S, Feiglin B, Hammarström L. Tooth ankylosis: clinical, radiographic and histological assessments. International journal of oral surgery. 1984;13:423-31.

31. Maslamani M, Almusawi A, Joseph B, Gabato S, Andersson L. An experimental model for studies on delayed tooth replantation and ankylosis in rabbits. Dental Traumatology. 2016;32:443-9.

32. Urist M. Bone histogenesis and morphogenesis in implants of demineralized enamel and dentin. Journal of oral surgery (American Dental Association: 1965). 1971;29:88.

33. Mordenfeld A, Hallman M, Lindskog S. Tissue reactions to subperiosteal onlays of demineralized xenogenous dentin blocks in rats. Dental Traumatology. 2011;27:446-51.

34. Machado M, Souza A, Araujo V. Histological evaluation of the osteoinduction capability of human dentine. International endodontic journal. 2006;39:855-9.

35. Caballero M, Morse JC, Halevi AE, Emodi O, Pharaon MR, Wood JS, et al. Juvenile Swine Surgical Alveolar Cleft Model to Test Novel Autologous Stem Cell Therapies. Tissue Eng Part C Methods: Mary Ann Liebert, Inc. 140 Huguenot Street, 3rd Floor New Rochelle, NY 10801 USA; 2015. p. 898-908.

36. el-Bokle D, Smith SJ, Germane N, Sharawy M. New technique for creating permanent experimental alveolar clefts in a rabbit model. Cleft Palate Craniofac J1993. p. 542-7.

37. El-Deeb M, Horswell B, Waite DE. A primate model for producing experimental alveolar cleft defects. J Oral Maxillofac Surg1985. p. 523-7. 
38. Gritli-Linde A. The mouse as a developmental model for cleft lip and palate research. Front Oral Biol: S. KARGER AG; 2012. p. 32-51.

39. Ishii Y. [Experimental study of secondary bone graft of alveolar clefts using bone morphogenetic protein (BMP)]. Kokubyo Gakkai zasshi The Journal of the Stomatological Society, Japan. 2001;68:111-24.

40. Liang L, Liu C. Trans-sutural distraction osteogenesis for alveolar cleft repair: an experimental canine study. Cleft Palate Craniofac J: Allen Press Publishing Services; 2012. p. 701-7.

41. Liao LS, Tan Z, Zheng Q, Wu J, Shi B, He X, et al. Animal experimental study on repairing alveolar clefts by using rectilinear distraction osteogenesis. Journal of plastic, reconstructive \& aesthetic surgery : JPRAS. 2009;62:1573-9.

42. Mostafa NZ, Doschak MR, Major PW, Talwar R. Reliable critical sized defect rodent model for cleft palate research. Journal of cranio-maxillo-facial surgery : official publication of the European Association for Cranio-Maxillo-Facial Surgery2014. p. 1840-6.

43. Nguyen PD, Lin CD, Allori AC, Ricci JL, Saadeh PB, Warren SM. Establishment of a critical-sized alveolar defect in the rat: a model for human gingivoperiosteoplasty. Plastic and reconstructive surgery. 2009;123:817-25.

44. Papadopoulos MA, Papadopulos NA, Jannowitz C, Boettcher P, Henke J, Stolla R, et al. Three-dimensional cephalometric evaluation of maxillary growth following in utero repair of cleft lip and alveolar-like defects in the mid-gestational sheep model. Fetal diagnosis and therapy. 2006;21:105-14.

45. Pilanci O, Cinar C, Kuvat SV, Altintas M, Guzel Z, Kilic A. Effects of Hydroxyapatite on Bone Graft Resorption in an Experimental Model of Maxillary Alveolar Arch Defects. Archives of Clinical and Experimental Surgery (ACES). 2013;2:170-5.

46. Raposo-Amaral CE, Kobayashi GS, Almeida AB, Bueno DF, Freitas FRdSe, Vulcano LC, et al. Alveolar osseous defect in rat for cell therapy: preliminary report. Acta Cir Bras2010. p. 313-7.

47. Sawada Y, Hokugo A, Nishiura A, Hokugo R, Matsumoto N, Morita S, et al. A trial of alveolar cleft bone regeneration by controlled release of bone morphogenetic protein: an experimental study in rabbits. Oral surgery, oral medicine, oral pathology, oral radiology, and endodontics. 2009;108:812-20.

48. Wu L-I, Zhao Y, Chen C. [Establishment of the animal model with unilateral alveolar cleft and its effect on the nose growth]. Zhonghua Zheng Xing Wai Ke Za Zhi2010. p. 39-42.

49. Xu Y, Sun J, Chen Z. Establishment of a rat model for alveolar cleft with bone wax. Journal of oral and maxillofacial surgery : official journal of the American Association of Oral and Maxillofacial Surgeons. 2015;73:733. e1-10.

50. Kamal M, Andersson L, Tolba R, Bartella A, Gremse F, Holzle F, et al. A rabbit model for experimental alveolar cleft grafting. Journal of translational medicine. 2017;15:50.

51. Gremse F, Doleschel D, Zafarnia S, Babler A, Jahnen-Dechent W, Lammers T, et al. Hybrid microCT-FMT imaging and image analysis. Journal of visualized experiments : JoVE. 2015:e52770.

52. Gremse F, Stark M, Ehling J, Menzel JR, Lammers T, Kiessling F. Imalytics Preclinical: Interactive Analysis of Biomedical Volume Data. Theranostics. 2016;6:328-41.

53. Kim J-H, Moon H-J, Kim T-H, Jo J-M, Yang SH, Naskar D, et al. A novel in vivo platform for studying alveolar bone regeneration in rat. Journal of tissue engineering. 2013;4:2041731413517705.

54. Puumanen K, Kellomaki M, Ritsila V, Bohling T, Tormala P, Waris T, et al. A novel bioabsorbable composite membrane of Polyactive 70/30 and bioactive glass number 13--93 in repair of experimental maxillary alveolar cleft defects. Journal of biomedical materials research Part B, Applied biomaterials. 2005;75:25-33.

55. Takano-Yamamoto T, Kawakami M, Sakuda M. Defects of the rat premaxilla as a model of alveolar clefts for testing bone-inductive agents. J Oral Maxillofac Surg1993. p. 887-91.

56. KolkA, Handschel J, Drescher W, Rothamel D, Kloss F, Blessmann M, et al. Current trends and future perspectives of bone substitute materials - from space holders to innovative biomaterials. Journal of cranio-maxillo-facial surgery : official publication of the European Association for Cranio-Maxillo-Facial Surgery. 2012;40:706-18. 
57. Schultze-Mosgau S, Nkenke E, Schlegel AK, Hirschfelder U, Wiltfang J. Analysis of bone resorption after secondary alveolar cleft bone grafts before and after canine eruption in connection with orthodontic gap closure or prosthodontic treatment. Journal of oral and maxillofacial surgery. 2003;61:1245-8.

58. Daculsi G. Biphasic calcium phosphate concept applied to artificial bone, implant coating and injectable bone substitute. Biomaterials. 1998;19:1473-8.

59. Lindgren C, Sennerby L, Mordenfeld A, Hallman M. Clinical histology of microimplants placed in two different biomaterials. International Journal of Oral and Maxillofacial Implants. 2009;24:1093-100.

60. Hallman M, Thor A. Bone substitutes and growth factors as an alternative/complement to autogenous bone for grafting in implant dentistry. Periodontology 2000. 2008;47:172-92.

61. Mordenfeld A, Hallman M, Johansson CB, Albrektsson T. Histological and histomorphometrical analyses of biopsies harvested 11 years after maxillary sinus floor augmentation with deproteinized bovine and autogenous bone. Clinical Oral Implants Research. 2010;21:961-70.

62. Ducheyne P, Radin S, King L. The effect of calcium phosphate ceramic composition and structure on in vitro behavior. I. Dissolution. Journal of Biomedical Materials Research Part A. 1993;27:25-34.

63. Gauthier O, Bouler J-M, Aguado E, Legeros R, Pilet P, Daculsi G. Elaboration conditions influence physicochemical properties and in vivo bioactivity of macroporous biphasic calcium phosphate ceramics. Journal of Materials Science: Materials in Medicine. 1999;10:199-204.

64. Schwartz C, Liss P, Jacquemaire B, Lecestre P, Frayssinet P. Biphasic synthetic bone substitute use in orthopaedic and trauma surgery: clinical, radiological and histological results. Journal of Materials Science: Materials in Medicine. 1999;10:821-5.

65. Jensen SS, Broggini N, Hjørting-Hansen E, Schenk R, Buser D. Bone healing and graft resorption of autograft, anorganic bovine bone and $\beta$-tricalcium phosphate. A histologic and histomorphometric study in the mandibles of minipigs. Clinical oral implants research. 2006;17:237-43.

66. Andersson L, Bodin I, Sörensen S. Progression of root resorption following replantation of human teeth after extended extraoral storage. Dental Traumatology. 1989;5:38-47.

67. Trope M. Avulsion of permanent teeth: theory to practice. Dental Traumatology. 2011;27:281-94.

68. Malmgren B. Ridge preservation/decoronation. Journal of endodontics. 2013;39:S67-S72.

69. Malmgren B, Tsilingaridis G, Malmgren O. Long-term follow up of 103 ankylosed permanent incisors surgically treated with decoronation-a retrospective cohort study. Dental Traumatology. 2015;31:184-9.

70. Ike M, Urist MR. Recycled dentin root matrix for a carrier of recombinant human bone morphogenetic protein. Journal of Oral Implantology. 1998;24:124-32.

71. Steiglitz BM, Ayala M, Narayanan K, George A, Greenspan DS. Bone morphogenetic protein-1/Tolloid-like proteinases process dentin matrix protein-1. Journal of Biological Chemistry. 2004;279:980-6.

72. Morris ML. The implantation of decalcified human dentin and cementum into the subcutaneous tissues of the rat. Journal of periodontal research. 1967;2:273-81.

73. Directive C. 86/609/EEC of 24 November 1986 on the approximation of laws, regulations and administrative provisions of the Member States regarding the protection of animals used for experimental and other scientific purposes. Off J Eur Commun. 1986;29:L358. 



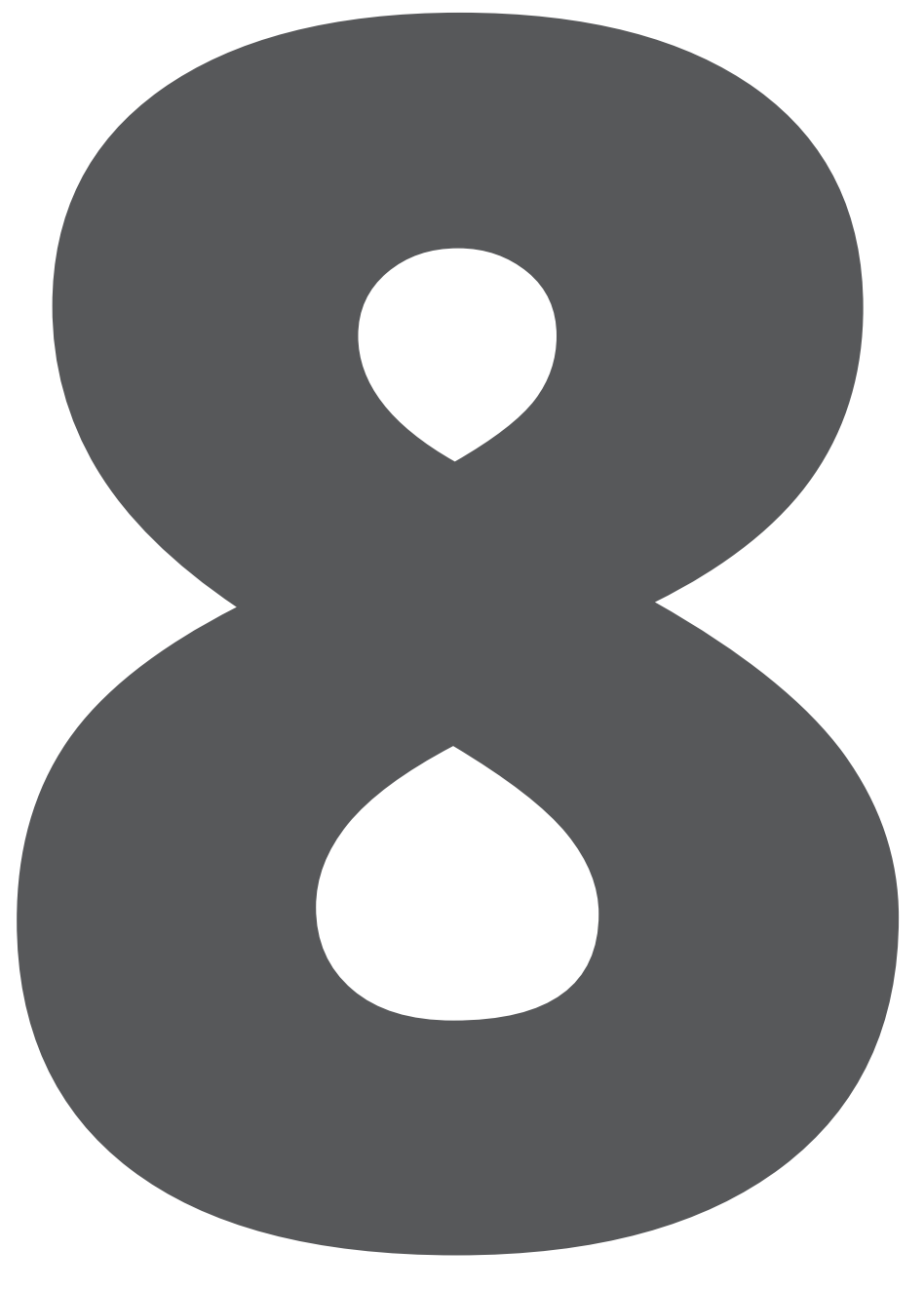




\section{Epilogue \\ General Discussion and Future Perspectives}





\section{EPILOGUE}

\section{Dentofacial Deformities - Global Healthcare Burden}

Treatment of alveolar cleft deformities requires a thorough understanding of the local tissue environment, the expected functional need and an elaborate appraisal of future oral rehabilitation options, mainly consisting of biomechanical orthodontic therapy or endosseal dental implant placement to restore occlusion. The clinical manifestation of these deformities extends beyond the deformation of the facial skeleton. An increasing number of epidemiological and public health studies have investigated this topic to reveal the burden of this deformity on society at large. Given the multi-stage repair required in genetically determined deformities, the social and behavioural ramification must be evaluated over an extended time frame from early childhood to adulthood. Not to mention, patients with cleft lip and palate deformities presenting with complex orofacial malformations, which require sequential treatment and interdisciplinary care among several specialities, cause a tremendous burden on the family, the society, and the healthcare system. Throughout our study, we found an increase in healthcare resources being allocated for the improvement of patients with cleft lip and palate across all continents. There seems to be consensus, that improving the health resources for patients with this congenital deformity can provide a positive contribution to the society overall, when evaluating the condition from a psychological and economical standpoint. The funding of medical research to improve the clinical outcomes for novel interventional therapies in this discipline seems to be on the rise, given the increased number of clinical and preclinical studies exploring the whole spectrum of medical technology from utilizing biocompatible materials to biologic active materials and stem cell therapies.

\section{Current Bone Grafting Options and \\ Technological Advancements in the Field of Biomaterials}

Our systematic review revealed a plethora of autogenous and bone substitute materials being used in humans for alveolar cleft bone grafting. We limited our analysis to studies reporting standardized outcomes to improve analysis and reportability. The majority of the cases were treated with autogenous bone, being the current gold standard in bone grafting, yet multiple types of donor bone were utilized. Many comparative studies were also noted from several decades back, which points out to the earlier recognition for the need to advance this field of therapeutic medicine. There was plausible evidence in the literature suggesting that autogenous bone, other than the iliac crest, can be used with success in bone grafting and resulted in better volume maintenance. In particular calvarial and symphysis bone grafts showed a promising outcome. This improvement of graft volume in craniofacial grafting could be related to the origin of calvarial and symphyseal bone, which is intramembranous in nature, similar to the craniofacial skel- 
eton. The varying outcome of grafting success obtained by each bone source provides hope for the use of other bone grafts to further improve the graft stability and longterm volume retention. In our cadaveric observational study evaluating bone specimens from various anatomical locations, we found significantly higher structural bone indices including bone mineral density in bone specimens obtained from the calvarium, mandibular ramus, mandibular symphysis, and the tibia when compared to the iliac crest bone and maxillary tuberosity. This is also reported in the literature and some of these bone grafts with better structural indices are currently being used in multiple centers with expertise in bone grafting.

In our systematic review, we also analyzed the utilization of various bone grafting substitutes; these materials ranged from growth factors, hematogenic factors, processed allogenic or xenogenic bone, tissue-engineered cells on scaffolds, to various alloplastic materials like calcium phosphate, calcium sulfate, bio-polymers, nanocomposites, and bio-glass. Our compiled meta-analysis showed no significant difference in bone filling capacity of bone substitute materials when compared to autogenous bone grafts. This shows promising results with regard to novel bone replacement materials being utilized in alveolar bone grafting. Further interventional studies testing more biomaterials for grafting are needed to improve our understanding of graft healing. Calcium phosphate biomaterials have shown great success in grafting, and have been used as a solo material, or in combination with other bone materials in alveolar cleft bone grafting. Dentin, on the other hand, was also used in several interventional and clinical studies as a grafting replacement material with encouraging results. However, there were no studies in the literature with composite Dentin/ß-TCP mixture as bone grafting materials.

\section{Experimental Animal Testing - The Cornerstone of Translational Medicine}

Even though the current literature showed the availability of many animal models with alveolar cleft defects simulating those in humans for testing bone grafting materials, some models were either difficult or costly to create, small in its size, not reproducible, or the animal was not suitable for our purpose of study. Earlier models have mainly used a direct, one stage, approach. To achieve a more valid situation, a proper three-dimensional skeletal platform has to be reconstructed with viable bone and cellular components to allow for a dynamic and physiologic reconstruction of the maxillary arch continuity. The development of an animal model in New Zealand White rabbits proved to be effective in creating a robust model. It can reliably be used for alveolar cleft grafting interventions and would result in normal healing and assessable bone regeneration by $\mu \mathrm{CT}$ or histomorphometry parameters, as we saw it in our 16 animals. In addition, we were successful in performing both the alveolar cleft creation surgery and the cleft exposure with interventional grafting under intravenous anesthesia and without the need for endotracheal intubation. 
Our animals tolerated both procedures well without any perioperative or postoperative complications, and were fed, alive, and functioned normally until the end of the study. This makes this animal model valid for reproducible testing, useful for short operations, and does not pose any major risks to the animals, which is attributed mostly to the short duration of the procedure and maintenance of the nasal mucosa. Standardization of the defect size seems to be difficult to achieve due the rapid healing phase after the cleft creation surgery. Further work needs to be done to standardize the size of these defects to eliminate bias during interventional testing.

The calvarial critical-size defects proved itself to be an effective tool in assessing bone regeneration under standardized conditions. We have successfully utilized this model to assess the osteogenic potential for our intervention in combination with the cleft defects. This adds scientific value to the experiment being tested in two separate sites, enabling a better evaluation of the healing potential of the testing materials in two anatomically different locations with distinct local tissue environment and biologic interactions.

\section{Dentin and B-TCP - A synergic Outcome of Bone Regeneration}

In both, the calvarial defect and the alveolar cleft model, the in-situ formed dentin/ß-TCP composite graft was successfully incorporated with superior bone formation to B-TCP alone. In addition, the volume of the residual grafts was significantly more in comparison to ß-TCP alone, which was mostly resorbed. The inflammatory reaction was minimal in both groups, which further improved the bone healing process. By utilizing the putty form of ß-TCP/HA, we were able to obtain superior surgical grafting of the material, that was non-setting, and easily mouldable into the defects without dimensional instability. The composite did not actively bind to the local bone, however, the formability of the graft made it easier to handle the dentin particles and keep the graft in a dimensionally stable form. It seems to be critical to stabilize the graft and prevent its movement. Previous studies have reported increasing fibrous encapsulation of the grafts which was mostly due to graft instability and micro-mobility, which we succeeded to eliminate by using the putty formulation.

Dentin is human tissue that is widely available, especially by extraction for orthodontic reasons. Its cost is minimal, and can easily be processed. However, it still needs to be treated like other processed allogenic tissues and must be properly prepared for use as

grafting material. Further work needs to be conducted to evaluate the effect of various sterilization techniques, including gamma radiation, on its healing characteristics. 


\section{Future Direction, Hopes, and Aims}

In our test setting, we were able to test the feasibility of dentin/ß-TCP in an injectable mixed form as an effective bone grafting substitute in two anatomical subsites. This study will prompt further investigations on the dentin processing techniques, and concentrations in the injectable ß-TCP/HA putty mixture to achieve an effective, dimensionally balanced graft. The addition of the dentin chips to the graft material seems to enhance bone formation, and provides better graft volume, because of its low rate of resorption and inherent osetoconduction potential. This would allow for the development of an affordable bone graft substitute material that would overcome the drawback of autogenous bone grafting. We propose that establishing a decalcified dentin tissue bank would help further clinical research and development of better grafting materials. 



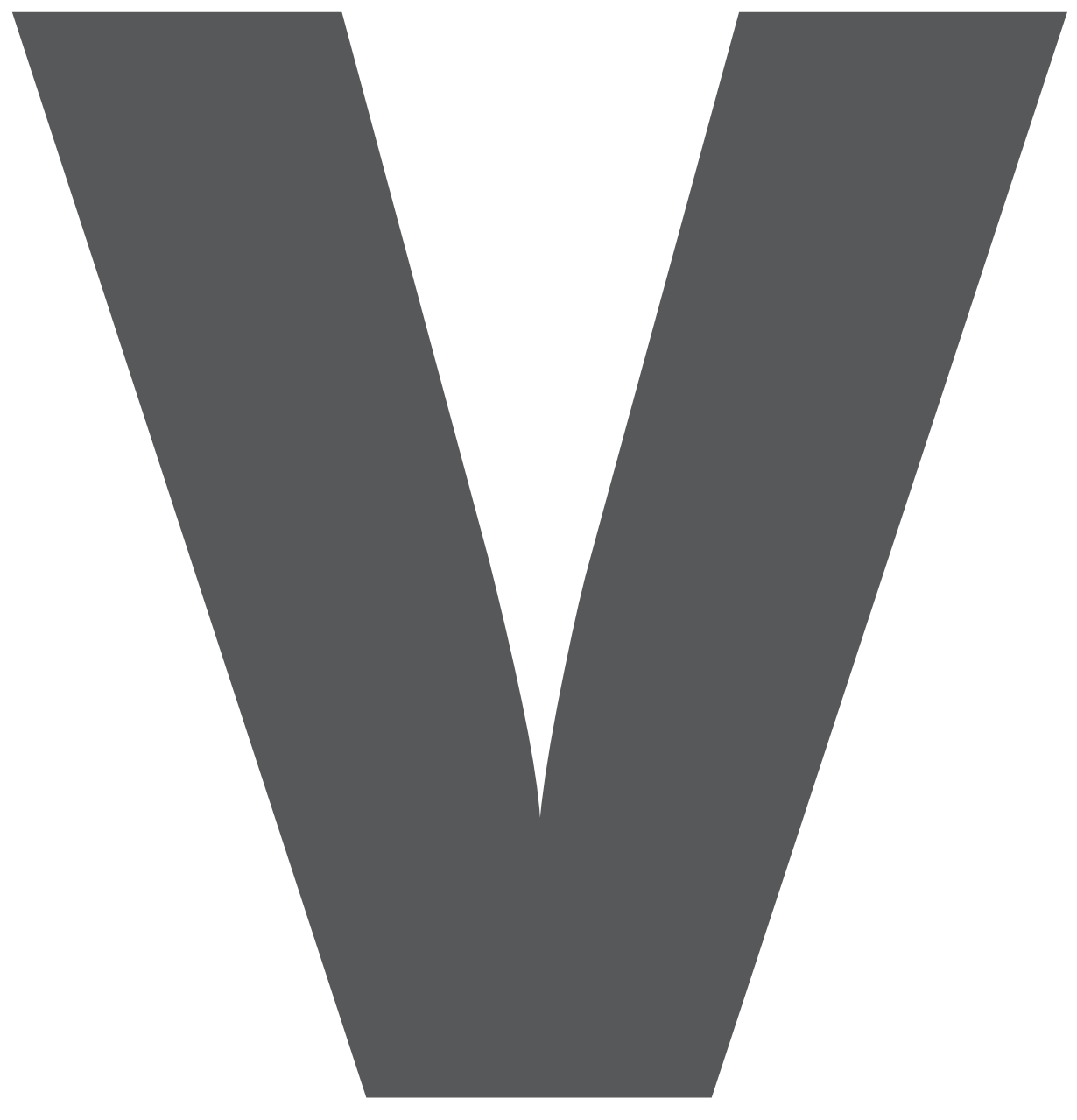


Valorisation Addendum 



\section{VALORISATION ADDENDUM}

Cleft lips and palates are the most common congenital human facial deformities, which can affect up to 1/500 new-borns depending on the geographic region and the ethnic variation. 1,2 Epidemiological reports from European registries on orofacial clefts stratified by country demonstrated major differences in the prevalence of cleft lip and palate across different European countries ranging from 2 per 1,000 births in Northern Europe to 1 in 1,000 births in Italy. ${ }^{1,2}$ The highest prevalence of non-syndromic/non-chromosomal cleft lip and palate was noted to be in Germany, Denmark, and the Netherlands with a prevalence around 2 per 1,000 births, whereas the lowest prevalence was recorded in Italy, Spain, and Portugal with less than 1 per 1,000 births. 1,2 The ramifications of these craniofacial malformations are not limited to its aesthetic disfigurements, but extend far beyond to directly affect patient's quality of living and their functional capacity in speaking, hearing, swallowing, chewing and breathing. This constellation of clinical impairments prompts a comprehensive treatment planning approach, and a complex interdisciplinary care to address all anatomical deficiencies and symptoms, including nursing, corrective surgery, otolaryngology, speech therapy, audiology, psychological and genetic counselling, orthodontics and dental treatment. Most children undergo several primary and secondary corrective surgeries starting soon after birth, their late teenage years and their adulthood in order to correct these multi-faceted craniofacial malformations. ${ }^{3}$ Therefore, the treatment of this orofacial malformation prompts a significant economic burden on families, the society, and the healthcare system in general. Many highly specialized centers have been established to optimize treatment protocols and monitor clinical outcomes.

Nevertheless, these deformities continue to inflict a life-long significant psychological impact on the patients and their families, especially in issues related to an altered body image. Several studies in the literature have evaluated the psychological impact on patients with cleft lip and palate deformities, and reported heterogeneous psychological disorders affecting self-attributes in children as well as in adults, including: anxiety, behavioural disturbances, inferior cognitive development, depression and dissatisfaction with body and face image. 4, 5 Moreover, further studies reported significant impact on interactional relationships of the affected patients, such as higher levels of sibling rivalry, along with internal feeling of embarrassment, guilt, and neglect. ${ }^{5}$ There is also clinical evidence that these facial disfigurements may interfere with the mother-child bonding, which is considered to be an important determinant of personality and psychological adjustment later in adult life. ${ }^{3}$ This facial malformation also affect the dynamics of verbal and non-verbal communication, through phonation and facial expression, and the interaction with the family, friends, and the society, and thus has also been described as a "social pathology". ${ }^{6}$ 
Thus, disfigurement of the maxilla and the dental arch presents only one aspect of the life-long suffering of children with cleft lip and palate. However, the manifestation of an alveolar cleft defect is presented during a critical phase of the child's development during school age, and a time of intense social interactions with the surrounding community. For children, school is the first place for open social interactions with others, and it plays an essential role in the social integration process and formation of the child's personality. ${ }^{6}$ By correcting the alveolar cleft deformity during the childhood years, and establishing a favourable dental anatomy, this would decrease the functional limitation of the patient, improve their oral rehabilitation, increase their social acceptance among peers, and normalize their facial appearance and their interactional abilities which would hopefully result in improvement of the overall psychological status of these patients.

By improving the methods of alveolar bone grafting, which is carried during school age, and decreasing the surgical morbidity and improving its outcomes, we would positively affect these children's experiences and promote their psychological wellness as well. However, bone harvesting may be difficult in small children. The biotechnology market has been responsive to these demands in recent years, and played a vital part in translating basic sciences from bench-side to bed-side clinical applications. Continuous testing of novel bone grafting substitutes has thus led to optimisation of bone grafting techniques in various disciplines. In our studies, we propose the utilization of autogenous or xenogenic dentin as an effective adjunct to existing bone substitute materials to maintain volume retention and a stable filling capacity over a long-term period. The feasibility of implementing this in clinical practice sounds promising, given the ease of collection of this natural tissue. 


\section{REFERENCES}

1. Mossey PA, Modell B. Epidemiology of oral clefts 2012: an international perspective. Cleft Lip and Palate: Karger Publishers; 2012. p. 1-18.

2. Allam E, Stone C, Windsor L. Cleft lip and palate: etiology, epidemiology, preventive and intervention strategies. Anat Physiol. 2014;4:1-6.

3. Turner S, Rumsey N, Sandy J. Psychological aspects of cleft lip and palate. The European Journal of Orthodontics. 1998;20:407-15.

4. Grollemund B, Galliani E, Soupre V, Vazquez M, Guedeney A, Danion A. The impact of cleft lip and palate on the parent-child relationships. Archives de pediatrie: organe officiel de la Societe francaise de pediatrie. 2010;17:1380-5.

5. Stock NM, Stoneman K, Cunniffe C, Rumsey N. The Psychosocial Impact of Cleft Lip and/or Palate on Unaffected Siblings. The Cleft Palate-Craniofacial Journal. 2016;53:670-82.

6. Lorot-Marchand A, Guerreschi P, Pellerin P, Martinot V, Gbaguidi C, Neiva C, et al. Frequency and socio-psychological impact of taunting in school-age patients with cleft lip-palate surgical repair. International journal of pediatric otorhinolaryngology. 2015;79:1041-8. 


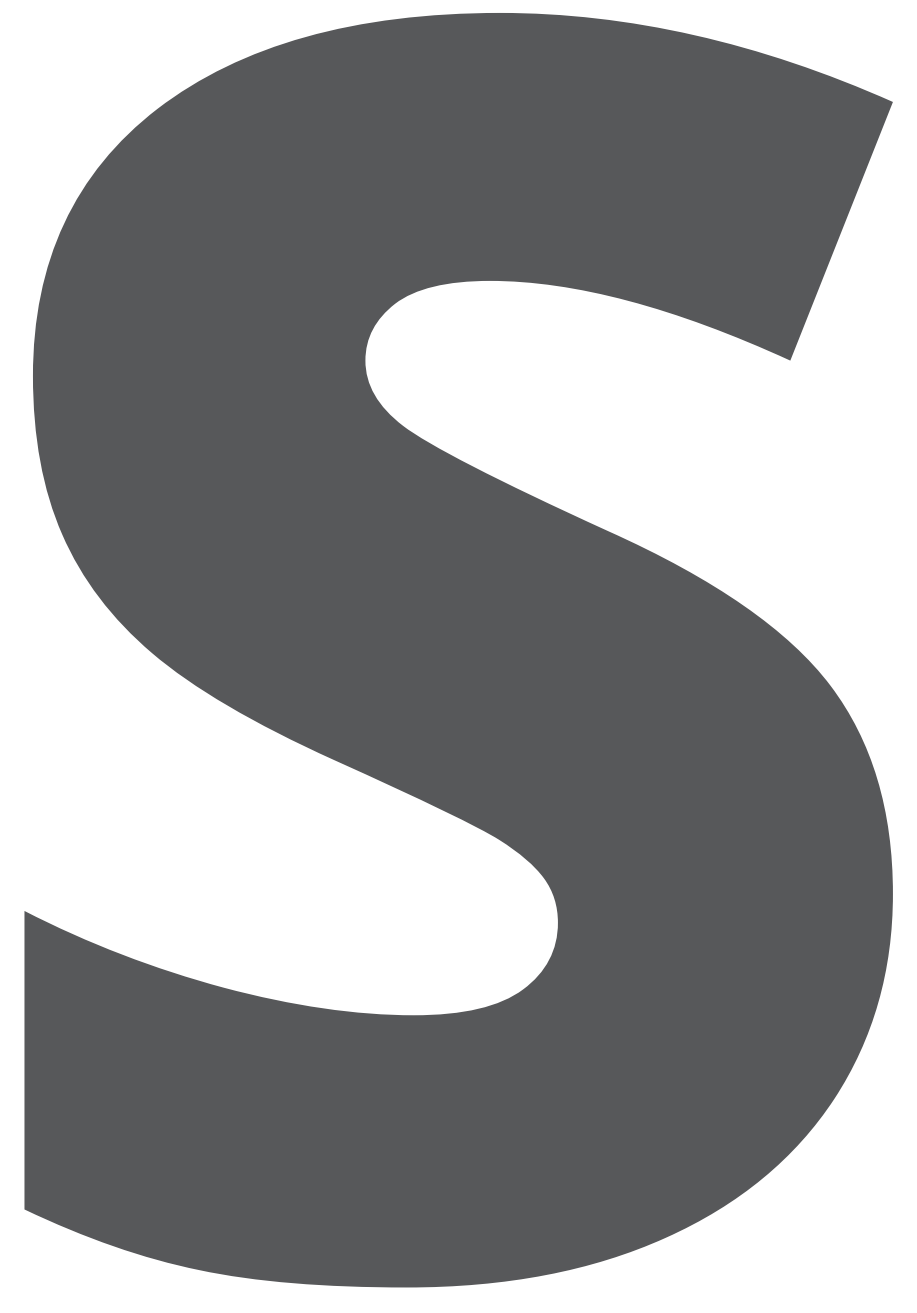


Summary 



\section{SUMMARY}

The comprehensive objective of our studies was to conduct a methodological analysis of the various grafting materials available in alveolar bone grafting in order to improve the overall treatment outcomes of alveolar cleft repair in children with cleft lip and palate.

Chapter 1 The introduction describes the clinical perspectives of cleft lip and palate and the epidemiology. Cleft lip and palate is a congenital malformation that can be manifested either as syndromic or non-syndromic deformity. There is a regional and ethnic predilection for increased prevalence of this malformation among newborns in different parts of the world. However, the exact pathology and mechanism for developing this malformation remains unclear. The malformation results in a structural deficiency in the upper jaw significantly affecting the formation of a continuous dental arch leading to missing teeth, and difficulties in speaking and swallowing. This has a great impact on the patient's quality of life from birth into adulthood. Our aim was to investigate novel options to repair the skeletal deformity as a result of cleft and palate in a biologically reliable and feasible animal model.

In Chapter 2 a comprehensive literature search on various grafting materials currently used for alveolar cleft repair was conducted. In order to assess the success of each grafting intervention, a clinical outcome was defined to evaluate the success of bone grafting. The percentage of bone filling of the cleft defect after grafting based on three-dimensional imaging modalities, which have been widely used for surgical assessment and planning in recent years, was used as the clinical outcome for the included studies. The search was performed electronically on MEDLINE, EMBASE, SCOPUS, Web of Science, and grey literature was conducted according to the Preferred Reporting Items for Systematic Reviews and Meta-Analyses (PRISMA) guidelines. A total of 25 studies were included in the meta-analysis to compare the volumetric bone filling percentage. After stratifying the intervention groups, a pooled analysis of 25 studies using autogenous bone revealed a statistically significant reduction in cleft volume equivalent to $62.0 \%$ bone fill $(95 \% \mathrm{Cl})$, in contrast to 10 studies using tissue-engineered material with a bone filling percentage of $68.7 \%(95 \% \mathrm{Cl})$. This demonstrates that there was no significant difference between autogenous bone grafts and novel tissue-engineered materials with regard to cleft filling capability. This was a noteworthy finding and indicated an increased efficacy of bone substitute materials as an effective option to autogenous bone grafts, which have long been considered the gold standard for grafting procedures.

In Chapter 3 we compared the three-dimensional morphometric microstructure in human cadaveric bone specimens taken from various commonly utilized donor sites for autogenous bone grafting. The morphometric analysis was conducted using $\mu \mathrm{CT}$, which generates 
high-resolution datasets of bone structures and calcifications making this modality versatile for microarchitecture analysis and quantification of bone. Six bone specimens were obtained from various anatomical sites from 10 human dentate cadavers. The specimens were scanned and volumetrically reconstructed. We utilized analytical software to analyze the bone mineral density and structural morphometric analysis using bone indices: relative bone volume, surface density, trabecular thicknesses, and trabecular separation for each group. Morphometric analysis revealed statistically significant differences in the bone mineral density and relative bone volume index in the calvaria, mandibular ramus, mandibular symphysis groups when compared to those in the iliac crest and maxillary tuberosity, suggesting higher bone quality in the former groups than in the latter; tibial specimens expressed variable results. These findings further support previous studies suggesting that autogenous bone from different donor sites expresses variable bone quality and structural characteristics, with variable levels of success and healing outcome.

In Chapter 4 we investigated whether xenogenic dentin particles inserted into the marrow space of rabbit tibia, a space where there is no solid bone tissue initially, would contribute to new bone formation. We knew from previous interventional studies that dentin possesses bone-forming properties and may possibly also be used as a bone augmentation material prior to implant placement. This phenomenon is seen in cases of dentoalveolar ankylosis, in which exposed dentin results in osseous replacement and formation of new bone. To test this hypothesis, dentin chips from human teeth were inserted into tibias of ten New Zealand White rabbits and histological processing was performed after 6 months. Bone formation was seen to a larger extent on dentin grafts located close to the native tibial bone wall and there was only minor inflammation. This was suggestive that dentin promotes new bone formation when located close to native cortical bone and may have a potential as a bone augmentation material.

In Chapter 5 we further conducted an interventional testing of dentin as potential grafting in combination with B-TCP, which is widely used for bone grafting in clinical practice, in the robust calvarial critical-size defects in ten New Zealand White rabbits. The purpose of this study was to evaluate bone healing in calvarial defects using two bone graft substitute materials; ß-TCP/HA versus composite non-demineralized xenogenic dentin with ß-TCP/ HA mixture. We analyzed the osteogenesis and healing patterns of the defects at eight weeks after grafting using $\mu C T f$. Quantitative analysis of bone mineral density (BMD) and bone volume fraction of the new bone formation (BVTV) in the defects were also evaluated. We showed that defects filled with composite non-demineralized xenogenic dentin with biphasic B-TCP expressed higher percentage bone volume fraction (BV/TV) than defects filled with ß-TCP only, suggesting a higher ratio of new bone formation. Histological analysis showed a significant percentage increase in bone formation and residual graft in the 
composite dentin/ß-TCP group after eight weeks. This suggests that composite xenogenic dentin with ß-TCP shows improved osteogenesis when compared to biphasic ß-TCP alone.

Chapter 6 describes a novel animal model in New Zealand White rabbits for creating alveolar cleft defects with a properly simulated clinical defect environment for tissue-engineered bone-substitute materials testing without compromising the health of the animal. Cleft creation surgery was aimed at creating a complete alveolar cleft with a wide bone defect with an epithelial lining (oral mucosa) overlying the cleft defect. The defect was surgically created using simple methodology without the need for intubation, and the defects were allow to heal for a eight-week period. Clinical examination and imaging analysis eight weeks after cleft creation surgery revealed the establishment of a wide skeletal defect extending to the nasal mucosa simulating alveolar clefts in all of the sixteen rabbits. The model allows the simulation of the cleft site environment in a biological model and can be used to evaluate various bone grafting materials with regard to efficacy of osteogenesis and healing potenital without compromising the health of the animal.

In Chapter 7 we conducted an interventional testing to evaluate the bone regeneration pattern and quantify bone formation after grafting the expimental alveolar clefts defects model in rabbits using composite xenogenic dentin and B-TCP in comparison to B-TCP alone. The unilateral alveolar cleft defects were created in sixteen New Zealand White rabbits according to the previously described methodology. Eight defects each were filled with ß-TCP, and composite xenogenic dentin with ß-TCP respectively. Bone regeneration of the healed defects was compared 8 weeks after intervention. Quantification of bone formation was analyzed using micro-computed tomography $(\mu \mathrm{CT})$ and histomorphometric analysis. $\mu \mathrm{CT}$ and histomorphometric analysis revealed that defects filled with composite dentin/ß-TCP showed statistically higher bone volume fraction, bone mineral density and percentage residual graft volume compared to ß-TCP alone. We also noted an improved surgical handling of the composite dentin/ß-TCP graft. In this chapter we showed that composite xenogenic Dentin/ß-TCP putty expresses enhanced bone regeneration compared to ß-TCP alone in the reconstruction of rabbit alveolar cleft defects.

In Chapter $\mathbf{8}$ the above findings were discussed in a comprehensive context. The majority of the alveolar cleft defects are being treated with autogenous bone, still being the gold standard in bone grafting, yet multiple types of donor bone were utilized. On the other hand, there has been an increasing application of tissue-engineered materials and various bone substitute materials in clinical practice in recent years. Recent interventional reports in the literature suggest improved clinical outcomes associated with these novel bone substitute materials. They may well be part of the daily clinical practice in the near future. Thus, there will be an increased need to perform interventional testings in reliable 
animal models to evaluate healing and efficacy of these materials, and to optimize their physical and biological characteristics, such as graft resorption rate, osteoconductivity, and osteoinductivity.

Bone grafting techniques seem to be in a dynamic phase of development. With the recent technological advances in the field of biomaterials, and the enormous improvement in the diagnostic and treatment modalities, we should expect a vast increase in the amount of bone grafting replacement biomaterials with the hope to overcome the shortcomings of conventional grafting techniques, mainly the limited donor supply, the need for an extra surgical procedure, risk of wound infection, blood loss, and increased pain and discomfort for the patients. Bone grafting is one of the most widely used routine procedures in clinical practice, in dental implantology, traumatology, oncology, craniofacial surgery, and cosmetic surgery. Alveolar bone grafting represents only one aspect of skeletal defects in addition to several other developmental anomalies, traumatic conditions, oncologic resections, infections, and pathological conditions leading to defects in the bony skeleton ultimately requiring bone grafting procedures. Optimizing the grafting modality in each of these conditions is the responsibility of scientists and health care professional involved in scientific research on grafting materials. 



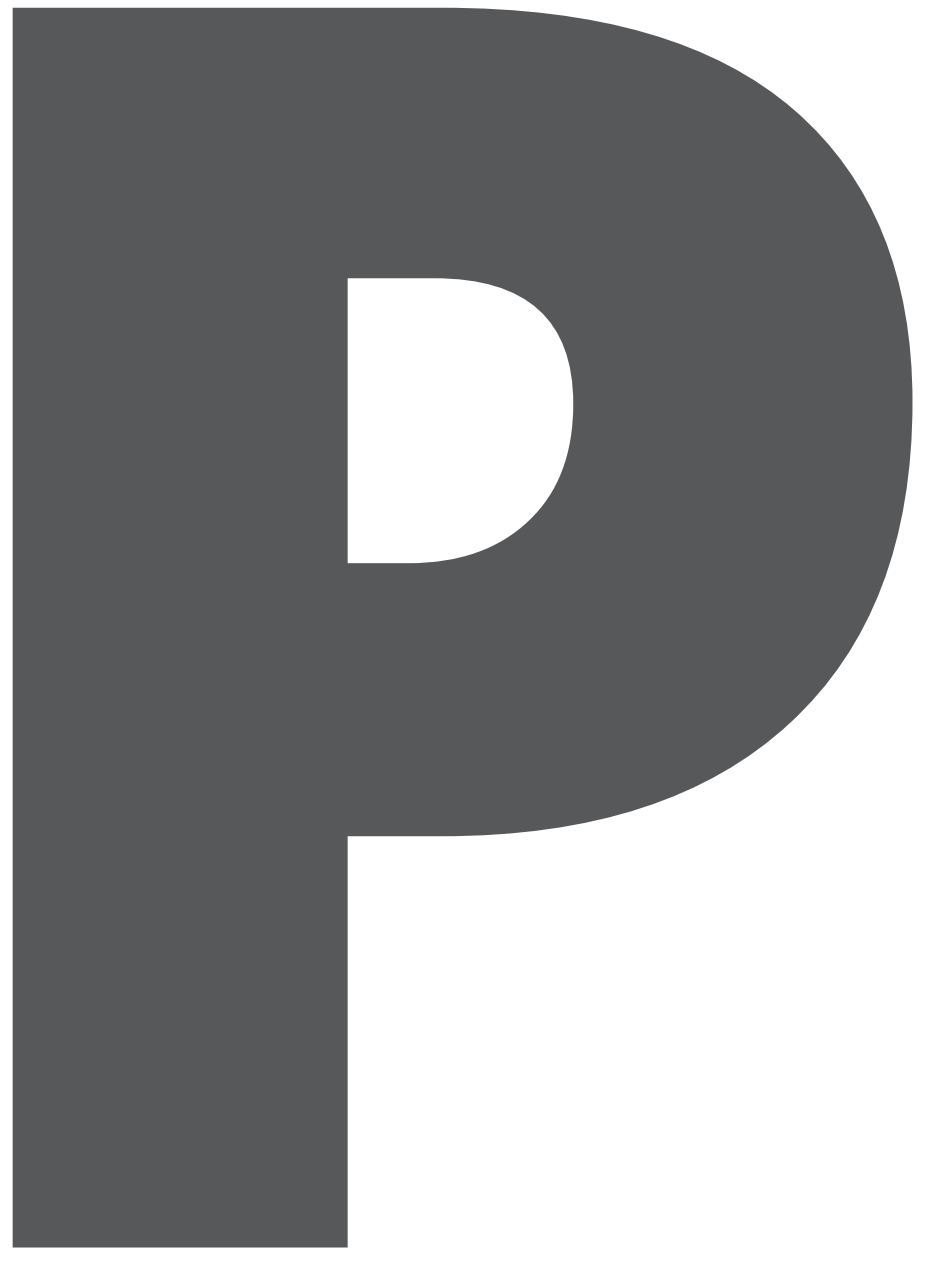


Publications 



\section{PUBLICATIONS}

1. M. Kamal, A.H. Ziyab, A. Bartella, D. Mitchell, A. Al-Asfour, F. Hölzle, P. Kessler, B. Lethaus: Volumetric comparison of autogenous bone and tissue-engineered bone replacement materials in alveolar cleft repair: a systematic review and meta-analysis. British Journal of Oral and Maxillofacial Surgery 05/2018; 56(6)., DOl:10.1016/j.bjoms.2018.05.007

2. Mohammad Kamal, Felix Gremse, Stefanie Rosenhain, Alexander K. Bartella, Frank Hölzle, Peter Kessler, Bernd Lethaus: Comparison of Bone Grafts From Various Donor Sites in Human Bone Specimens. Journal of Craniofacial Surgery 05/2018;, DOI:10.1097/ SCS.0000000000004586

3. Juliana-Theresa Schell, Andrea Petermann-Meyer, Anita Kloss-Brandstätter, Alexander K. Bartella, Mohammad Kamal, Frank Hölzle, Bernd Lethaus, Jan Teichmann: Distress thermometer for preoperative screening of patients with oral squamous cell carcinoma. Journal of Cranio-Maxillofacial Surgery 05/2018; 46(7)., DOI:10.1016/j. jcms.2018.04.022

4. A.K. Bartella, A.-K. Sander, M. Kamal, J. Steegmann, A. Kloss-Brandstätter, J. Teichmann, F. Hölzle, B. Lethaus: Preoperative assessment of the risk of postoperative death in patients with oral squamous cell carcinoma: a consideration beyond age, sex, and stage of cancer. DOI:10.1016/j.bjoms.2018.03.006

5. A K Bartella, C Lechner, M Kamal, J Steegmann, F Hölzle, B Lethaus: The safety of paediatric dentistry procedures under general anaesthesia. A five-year experience of a tertiary care center. European Journal of Paediatric Dentistry 03/2018; 19(1):44-48., DOI:10.23804/ejpd.2018.19.01.08

6. Alexander K. Bartella, Sebastian Lemmen, Aida Burnic, Anita Kloss-Brandstätter, Mohammad Kamal, Thomas Breisach, Frank Hölzle, Bernd Lethaus: Influence of a strictly perioperative antibiotic prophylaxis vs a prolonged postoperative prophylaxis on surgical site infections in maxillofacial surgery. Infection 12/2017 „, DOI:10.1007/s15010017-1110-4

7. Mohammad Kamal, Lars Andersson, Rene Tolba, Adel Al-Asfour, Alexander K. Bartella, Felix Gremse, Stefanie Rosenhain, Frank Hölzle, Peter Kessler, Bernd Lethaus: Bone regeneration using composite non-demineralized xenogenic dentin with beta-tricalcium phosphate in experimental alveolar cleft repair in a rabbit model. Journal of Translational Medicine 12/2017; 15(1):263., DOI:10.1186/s12967-017-1369-3 
8. Mohammad Kamal, Lars Andersson, Rene Tolba, Alexander Bartella, Felix Gremse, Frank Hölzle, Peter Kessler, Bernd Lethaus: A rabbit model for experimental alveolar cleft grafting. Journal of Translational Medicine 12/2017; 15(1)., DOI:10.1186/s12967017-1155-2

9. Alexander K. Bartella, Mohammad Kamal, Sean Berman, Timm Steiner, Dirk Frölich, Frank Hölzle, Bernd Lethaus: Role of Swallowing Function of Tracheotomised Patients in Major Head and Neck Cancer Surgery. Journal of Craniofacial Surgery 10/2017; DOI:10.1097/SCS.0000000000004099

10. Alexander K. Bartella, Mohammad Kamal, Jan Teichmann, Anita Kloss-Brandstätter, Timm Steiner, Frank Hölzle, Bernd Lethaus: Prospective comparison of perioperative antibiotic management protocols in oncological head and neck surgery. Journal of Cranio-Maxillofacial Surgery 04/2017; 45(7)., DOI:10.1016/j.jcms.2017.04.001

11. Alexander K. Bartella, Anita Kloss-Brandstätter, Mohammad Kamal, Jan Teichmann, Ali Modabber, Frank Hölzle, Bernd Lethaus: "Ilb or not IIb" - The Necessity of Dissection in Patients with Oral Squamous Cell Carcinoma. Journal of Cranio-Maxillofacial Surgery 08/2016; 44(10)., DOI:10.1016/j.jcms.2016.08.003

12. Stephan Christian Möhlhenrich, Mohammad Kamal, Florian Peters, Ulrike Fritz, Frank Hölzle, Ali Modabber: Bony contact area and displacement of the temporomandibular joint after high-oblique and bilateral sagittal split osteotomy: A computer-simulated comparison. British Journal of Oral and Maxillofacial Surgery 02/2016; 54(3)., DOI:10.1016/j. bjoms.2015.12.020

13. Stephan Christian Möhlhenrich, Ali Modabber, Mohammad Kamal, Ulrike Fritz, Andreas Prescher, Frank Hölzle: Three-dimensional effects of pterygomaxillary disconnection during surgically assisted rapid palatal expansion: A cadaveric study. Oral Surgery, Oral Medicine, Oral Pathology, Oral Radiology, and Endodontology 01/2016; 121(6)., DOl:10.1016/j.oooo.2015.12.013

14. Stephan Christian Möhlhenrich, Nicole Heussen, Mohammad Kamal, Florian Peters, Ulrike Fritz, Frank Hölzle, Ali Modabber: Influence of setback and advancement osseous genioplasty on facial outcome: A computer-simulated study. Journal of Cranio-Maxillofacial Surgery 10/2015; 43(10)., DOI:10.1016/j.jcms.2015.10.017 
15. Stephan Christian Möhlhenrich, Nicole Heussen, Mohammad Kamal, Ulrike Fritz, Frank Hölzle, Ali Modabber: Limitations of osseous genioplasty in relation to the displacement distance: A computer-based comparative study. 07/2015; 120(6)., DOI:10.1016/j. oooo.2015.06.040

16. Mohamad A Bitar, Mohammad Kamal, Charbel Rameh: A Vallecular Thyroglossal Duct Cyst Presenting with Persistent Unexplained Cough in a Toddler.

17. Adel Al-Asfour, Lars Andersson, Mohammad Kamal, Bobby Joseph: New bone formation around xenogenic dentin grafts to rabbit tibia marrow. Dental Traumatology 04/2013; 29(6)., DOI:10.1111/edt.12045

18. Mohamed A Bitar, Mohammad A Kamal, Lorice Mahfoud: Spontaneous sublingual haematoma: A rare entity. BMJ Case Reports 05/2012; 2012(may29 1)., DOI:10.1136/ bcr.12.2011.5367

19. George L Barnes, Kerri E Hebert, Mohammad H. Kamal, Amjad Javed, Thomas A Einhorn, Jane B Lian, Gary S Stein, Louis C Gerstenfeld: Fidelity of Runx2 activity in breast cancer cells is required for the generation of metastases-associated osteolytic disease. Cancer Research 08/2004; 64(13):4506-13., DOI:10.1158/0008-5472.CAN-03-3851

20. George L Barnes, Amjad Javed, Sylvan M Waller, Mohammad H Kamal, Kerri E Hebert, Mohammad Q Hassan, Akeila Bellahcene, Andre J Van Wijnen, Marian F Young, Jane B Lian, Gary S Stein, Louis C Gerstenfeld: Osteoblast-related transcription factors Runx2 (Cbfa1/AML3) and MSX2 mediate the expression of bone sialoprotein in human metastatic breast cancer cells. Cancer Research 06/2003; 63(10):2631-7. 


$$
\text { C }
$$




\section{Curriculum Vitae}





\section{CURRICULUM VITAE}

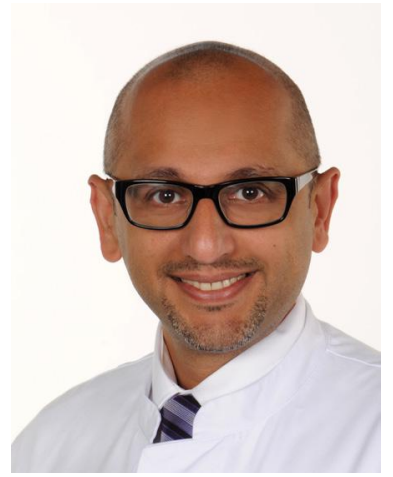

Mohammad Kamal was born in Kuwait in 1978 where he was raised and completed his primary and secondary education before moving to the United States on a merit scholarship in 1996 to pursue his university education. He first completed his Bachelor of Science B.Sc. degree with magna cum laude in medical sciences at Boston University School of Arts and Sciences in 2000, then completed his Doctor of Dental Medicine D.M.D. degree with magna cum laude and first rank among all dental graduates of Boston University School of Dental Medicine in 2004. Mohammad has received several awards and prizes during his studies in the United States including Boston University Dean's List 1997-2004, the American Dental Association/Dentsply Student Clinician Program Award, the Sigma Xi award and first place winner in scientific poster presentation at Boston University Science and Technology day, the American Association of Oral and Maxillofacial Surgeons Student Award, the Quintessence Award for Research Achievement, the Academy of Operative Dentistry Award, and the Omicron Kappa Upsilon Dental Award.

Afterwards he pursued further clinical training in Oral and Maxillofacial Surgery at the University of California San Francisco from 2006 to 2009 under the supervision of Professor Tony Pogrel before attending the American University of Beirut School of Medicine where he completed his medical studies and was awarded a Doctor of Medicine M.D. degree in 2011. Mohammad worked in Kuwait as a dentist from 2004 to 2006 and as a physician in different medical disciplines from 2012 to 2013 before moving to Germany to continue his surgical specialization in Oral and Maxillofacial Surgery at RWTH Aachen University Hospital in 2013 under supervision of Prof. Dr. Dr. Frank Hölzle, and was officially certified as a Specialist in Oral and Maxillofacial Surgery in Germany (Facharzt) in 2018. Mohammad is a member of the academic staff at Kuwait University Health Sciences Center, Department of Surgical Sciences, where he plans to return to and to work as an academic Oral and Maxillofacial surgeon. 


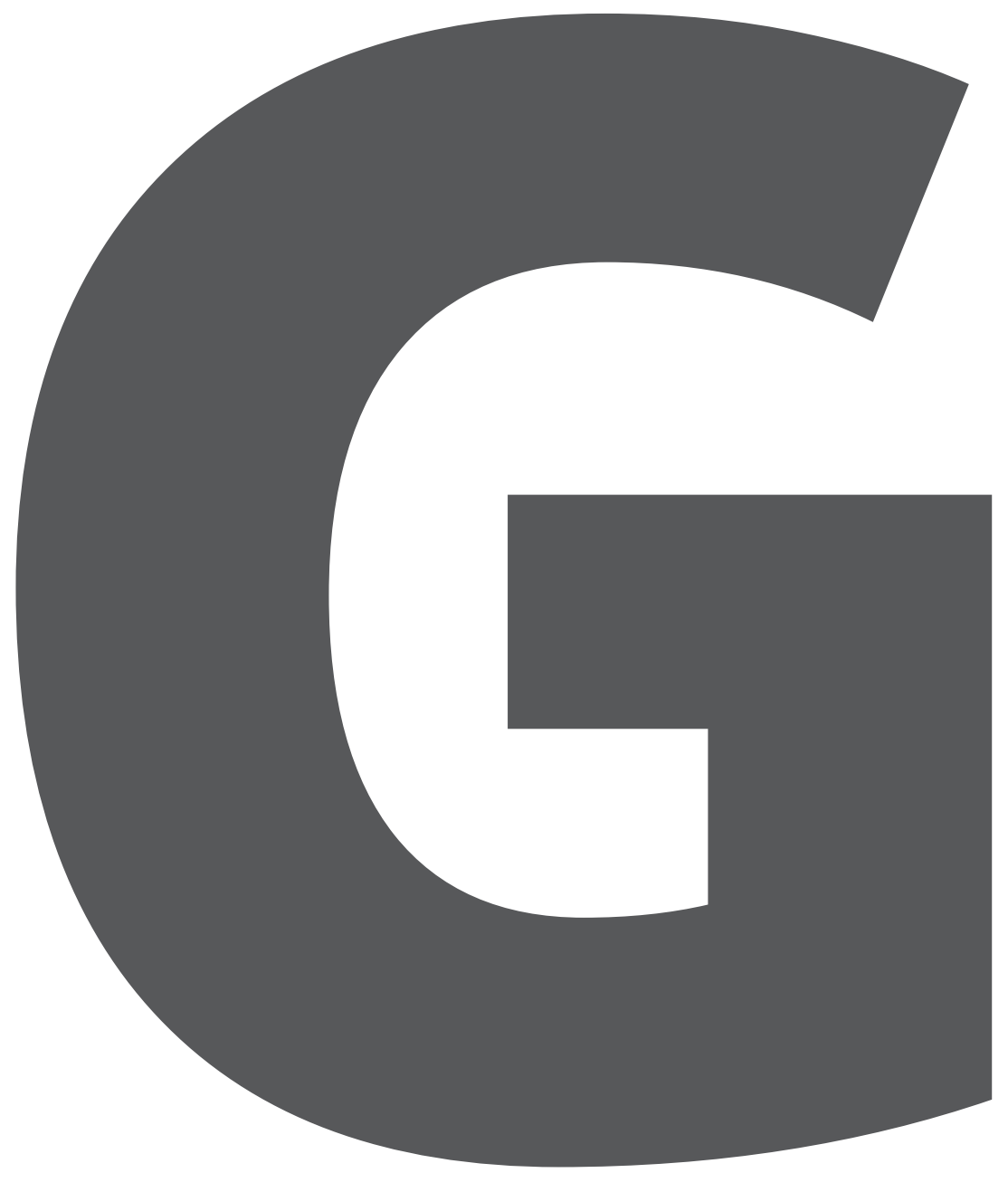


Gratitude 



\section{GRATITUDE}

I would like to express my most sincere gratitude and appreciation to Dr. Dr. Bernd Lethaus for his guidance, patience and encouragement throughout the development of the project. He definitely succeeded in making this doctoral thesis an enjoyable experience, for which I am enormously grateful. I would also like to thank Professor Frank Hölzle and the rest of his team at RWTH Aachen University Hospital for their assistance in helping the development of this combined PhD program with Maastricht University. His continuous belief in the advancement of healthcare science has provided me with utmost support to make this project possible.

I would like to thank Professor Peter Kessler for his help in making this collaborative PhD program between RWTH Aachen University Hospital and the Maastricht University Medical Center possible. His continuous guidance made it possible to fulfil my academic objectives during the PhD work promptly. I also had the privilege of working with his fellow doctors, researchers and staff at the Department of Cranio-Maxillofacial Surgery at Maastricht University, who were always kind and helpful to me.

I would also like to extend my deepest thanks to Professor Lars Andersson, who was instrumental in getting me started with research from earlier time and without whose help, guidance and patience, this thesis would not have been successful. Professor Andersson was always there to provide me with courage, believing in my potential, and supporting me to further advance my professional career. I greatly appreciate his unparalleled support.

I am greatly indebted to Professor Tony Pogrel from the University of California at San Francisco for his tremendous help during my early years of training in Oral and Maxillofacial Surgery, and his continuous support throughout the years. He has had a positive impact on me, which nourished the inspiration and dedication needed to pursue further clinical venture.

I am also sincerely grateful to Dr. Jawad Behbehani for his guidance, advice and help rendered to me throughout the years of my clinical training and graduate studies. Without his unconditional support, it would have been impossible to advance my career perspectives in the field of Oral and Maxillofacial Surgery.

I must also express my deepest gratitude to Dr. Adel Al-Asfour, Dean of Kuwait University Faculty of Dentistry, for the genuine and invaluable guidance he provided throughout my medical training. His leadership and vision in advancing future career aspirations in surgical corrections of facial deformities has greatly assisted me in my quest for knowledge. 
My mother and family have closely followed me from a distance through all of my education and provided me with the courage needed to keep on going. The completion of this thesis will mean a lot to them, and provide an inspiration for the younger members.

I dedicate this work to my loving wife and children, for supporting me throughout my academic endeavour, and bringing a colourful family life to our overseas living, and without whose love, affection and encouragement, this work would not have been possible. 


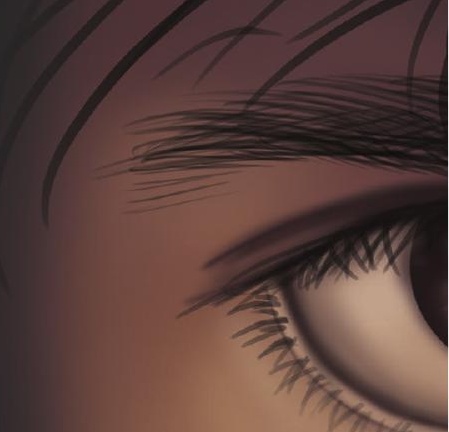

